\title{
On the mixed problem for the semilinear Darcy-Forchheimer-Brinkman PDE system in Besov spaces on creased Lipschitz domains
}

\author{
Robert Gutt $^{\mathrm{a}}$, Mirela Kohr ${ }^{\mathrm{a} *}$ Sergey E. Mikhailov ${ }^{\mathrm{b}}$ and \\ Wolfgang L. Wendland ${ }^{\mathrm{C}}$
}

The purpose of this paper is to study the mixed Dirichlet-Neumann boundary value problem for the semilinear DarcyForchheimer-Brinkman system in $L_{p}$-based Besov spaces on a bounded Lipschitz domain in $\mathbb{R}^{3}$, with $p$ in a neighborhood of 2 . This system is obtained by adding the semilinear term $|u| u$ to the linear Brinkman equation. First, we provide some results about equivalence between the Gagliardo and non-tangential traces, as well as between the weak canonical conormal derivatives and the non-tangential conormal derivatives. Various mapping and invertibility properties of some integral operators of potential theory for the linear Brinkman system, and well posedness results for the Dirichlet and Neumann problems in $L_{p}$-based Besov spaces on bounded Lipschitz domains in $\mathbb{R}^{n}(n \geq 3)$ are also presented. Then, employing integral potential operators, we show the well-posedness in $L_{2}$-based Sobolev spaces for the mixed problem of Dirichlet-Neumann type for the linear Brinkman system on a bounded Lipschitz domain in $\mathbb{R}^{n}(n \geq 3)$. Further, by using some stability results of Fredholm and invertibility properties and exploring invertibility of the associated Neumann-to-Dirichlet operator, we extend the well-posedness property to some $L_{p}$-based Sobolev spaces. Next we use the well-posedness result in the linear case combined with a fixed point theorem in order to show the existence and uniqueness for a mixed boundary value problem of Dirichlet and Neumann type for the semilinear Darcy-Forchheimer-Brinkman system in $L_{p}$-based Besov spaces, with $p \in(2-\varepsilon, 2+\varepsilon)$ and some parameter $\varepsilon>0$. Copyright (C) 0000 John Wiley \& Sons, Ltd.

Keywords: Semilinear Darcy-Forchheimer-Brinkman system; mixed Dirichlet-Neumann problem; $L_{p}$-based Besov spaces; layer potential operators; Neumann-to-Dirichlet operator; existence and uniqueness.

\section{Introduction}

Boundary integral methods are a powerful tool to investigate linear elliptic boundary value problems that appear in various areas of science and engineering (see, e.g., $[4,18,22,45,62]$ ). Among many valuable contributions in the field we mention the well-posedness result of the Dirichlet problem for the Stokes system in Lipschitz domains in $\mathbb{R}^{n}(n \geq 3)$ with boundary data in $L_{2}$-based Sobolev spaces, which have been obtained by Fabes, Kenig and Verchota in [23] by using a layer potential analysis. Also, Mitrea and Wright [61] obtained the well-posedness results for Dirichlet, Neumann and transmission problems for the Stokes system on arbitrary Lipschitz domains in $\mathbb{R}^{n}(n \geq 2)$, with data in Sobolev and Besov-Triebel-Lizorkin spaces. By using a boundary integral method, Mitrea and Taylor [62] obtained well-posedness results for the Dirichlet problem for the Stokes system on arbitrary Lipschitz domains on a compact Riemannian manifold, with boundary data in $L_{2}$. Their results extended the results of [23] from the Euclidean setting to the case of compact Riemannian manifolds. Continuing the study of [62], Dindoš and Mitrea [22] developed a layer potential analysis to obtain existence and uniqueness results for the Poisson problem for the

\footnotetext{
a Faculty of Mathematics and Computer Science, Babeș-Bolyai University, 1 M. Kogălniceanu Str., 400084 Cluj-Napoca, Romania.

${ }^{b}$ Department of Mathematics, Brunel University London, Uxbridge, UB8 3PH, United Kingdom.

'Institut für Angewandte Analysis und Numerische Simulation, Universität Stuttgart, Pfaffenwaldring 57, 70569 Stuttgart, Germany.

* Correspondence to: E-mail: mkohr@math.ubbcluj.ro
}

Contract/grant sponsor: The work of M. Kohr was supported by the "Scientific Grant for Excellence in Research", GSCE-30259/2015, of the Babes-Bolyai University. The work of S.E. Mikhailov and W.L. Wendland was supported by the grant EP/M013545/1DSM: "Mathematical Analysis of Boundary-Domain Integral Equations for Nonlinear PDEs" from the EPSRC, UK 
Stokes and Navier-Stokes systems on $C^{1}$ domains, but also on Lipschitz domains in compact Riemannian manifolds. Medková in [45] studied various transmission problems for the Brinkman system.

Due to many practical applications, the mixed problems for elliptic boundary value problems on smooth and Lipschitz domains have been also intensively investigated. Let us mention that Mitrea and Mitrea in [57] have proved sharp well-posedness results for the Poisson problem for the Laplace operator with mixed boundary conditions of Dirichlet and Neumann type on bounded Lipschitz domains in $\mathbb{R}^{3}$ whose boundaries satisfy a suitable geometric condition introduced by Brown [7], and with data in Sobolev and Besov spaces. Brown et al. [9] have obtained the well-posedness result of the mixed Dirichlet-Neumann problem for the Stokes system on creased Lipschitz domains in $\mathbb{R}^{n}(n \geq 3)$. In order to prove the desired well-posedness result, the authors reduced such a boundary value problem to a boundary integral equation, obtained useful Rellich-type estimates, and used the well-posedness result of the mixed Dirichlet-Neumann problem for the Lamé system that has been obtained in [8]. Costabel and Stephan in [19] analyzed mixed boundary value problems in polygonal domains by using a boundary integral approach. In $[13,15]$, direct segregated systems of boundary-domain integral equations equivalent to mixed boundary value problems of Dirichlet-Neumann type for a scalar second-order divergent elliptic partial differential equation with a variable coefficient, were analyzed in interior and exterior domains in $\mathbb{R}^{3}$ (see also [14] for the mixed problems with cracks and [48] for united boundary-domain integral equations). An interesting boundary integral equation method for a mixed boundary value problem of the biharmonic equation has been developed in [11]

Boundary integral methods combined with fixed point theorems have been focused on the analysis of boundary value problems for linear elliptic systems with nonlinear boundary conditions and for nonlinear elliptic systems with various (linear or nonlinear) boundary conditions. Recently, the authors in [33] have used a boundary integral method to obtain existence results for a nonlinear problem of Neumann-transmission type for the Stokes and Brinkman systems on Lipschitz domains in Euclidean setting and with boundary data in various $L_{p}$. Sobolev, or Besov spaces. The techniques of layer potential theory for the Stokes and Brinkman systems was used in [36] to analyze Poisson problems for semilinear generalized Brinkman systems on Lipschitz domains in $\mathbb{R}^{n}$ with Dirichlet or Robin boundary conditions and given data in Sobolev and Besov spaces. Boundary value problems of Robin type for the Brinkman and Darcy-Forchheimer-Brinkman systems in Lipschitz domains in Euclidean setting have been investigated in [35] (see also [34, 37]). An integral potential method for transmission problems with Lipschitz interface in $\mathbb{R}^{3}$ for the Stokes and Darcy-Forchheimer-Brinkman systems and data in weighted Sobolev spaces has been recently obtained in [32]. Transmission problems for the Navier-Stokes and Darcy-Forchheimer-Brinkman systems in Lipschitz domains on compact Riemannian manifolds have been recently analyzed in [39]. Well-posedness results for semilinear elliptic problems on Lipschitz domains in compact Riemannian manifolds have been obtained by Dindoš and Mitrea in [21]. Let us also mention that Russo and Tartaglione in $[67,68]$ used a double-layer integral method in order to obtain existence results for boundary problems of Robin type for the Stokes and Navier-Stokes systems in Lipschitz domains in Euclidean setting with data in Sobolev spaces. Maz'ya and Rossmann [42] obtained $L p$ estimates of solutions to mixed boundary value problems for the Stokes system in polyhedral domains. Taylor, Ott and Brown in [70] studied $L p$-mixed Dirichlet-Neumann problem for the Laplace equation in a a bounded Lipschitz domain in $\mathbb{R}^{n}$ with general decomposition of the boundary.

In this paper we analyze the mixed Dirichlet-Neumann boundary value problem for the semilinear Darcy-Forchheimer-Brinkman system in $L_{p}$-based Besov spaces on a bounded Lipschitz domain in $\mathbb{R}^{3}$, when the given boundary data belong to $L_{p}$ spaces, with $p$ in a neighborhood of 2 . This system is obtained by adding the semilinear term $|\mathbf{u}| \mathbf{u}$ to the linear Brinkman equation. First, we provide some results about equivalence between the Gagliardo and non-tangential traces, as well as between the weak canonical conormal derivatives and the non-tangential conormal derivatives. Various mapping and invertibility properties of some integral operators of potential theory for the linear Brinkman system, and well posedness results for the Dirichlet and Neumann problems in $L_{p}$-based Besov spaces on bounded Lipschitz domains in $\mathbb{R}^{n}(n \geq 3)$ are also presented. Based on these results we show the well-posedness result for the mixed problem of Dirichlet-Neumann type for the Brinkman system in a bounded domain in $\mathbb{R}^{n}(n \geq 3)$ with given data in $L_{2}$-based Sobolev spaces. Further, by using some stability results of Fredholm and invertibility properties, we extend the well-posedness property to the case of boundary data in $L_{p}$-based Sobolev spaces, with $p \in\left(\frac{2(n-1)}{n+1}-\varepsilon, 2+\varepsilon\right) \cap(1,+\infty)$, for some $\varepsilon>0$. The main idea for showing this property is the invertibility of an associated Neumann-to-Dirichlet operator, inspired by the approach developed by Mitrea and Mitrea in [57]. Next we use the well-posedness result in the linear case combined with a fixed point theorem in order to show the existence and uniqueness in $L_{p}$-based Besov spaces for a mixed boundary value problem of Dirichlet and Neumann type for the semilinear Darcy-Forchheimer-Brinkman system in a Lipschitz domain in $\mathbb{R}^{3}$, when the boundary data belong to some $L_{p}$ spaces, with $p \in(2-\varepsilon, 2+\varepsilon)$ and some parameter $\varepsilon>0$. The motivation of this work is based on some practical applications, where the semilinear Darcy-Forchheimer-Brinkman system describes the motion of viscous incompressible fluids in porous media. A suggestive example is given by a sandstone reservoir filled with oil, or the convection of a viscous fluid in a porous medium located in a bounded domain, where a part of the boundary is in contact with air and the remaining part is a solid surface or the interface with another immiscible material or fluid. All these problems are well described by the Brinkman system, the semilinear Darcy-Forchheimer-Brinkman system, or by the Darcy-Forchheimer-Brinkman system, the latter of these systems containing both the nonlinear convective term $(\mathbf{u} \cdot \nabla) \mathbf{u}$ and the semilinear term $|\mathbf{u}| \mathbf{u}$. For further details we refer the reader to the book by Nield and Bejan [65] (see also the theoretical and numerical approach in $[25,26])$.

It is supposed that the methods presented in this paper can be developed further, to analyze also the nonlinear boundary-domain integro-differential equations, e.g., the ones formulated in $[49,50]$ for some quasi-linear boundary value problems. 


\section{Functional setting and useful results}

The purpose of this section is to provide main notions and results used in this paper. We recall the definition of a bounded Lipschitz domain and give a short review of the involved Sobolev, Bessel potential and Besov spaces. Also we present the main properties of the layer potential operators for the Stokes and Brinkman systems in Lipschitz domains in $\mathbb{R}^{n}$.

For any point $x=\left(x_{1}, x_{2}, \ldots, x_{n}\right) \in \mathbb{R}^{n}$, we use the representation $x=\left(x^{\prime}, x_{n}\right)$, where $x^{\prime} \in \mathbb{R}^{n-1}$ and $x_{n} \in \mathbb{R}$. First, we recall the definition of Lipschitz domain (cf., e.g., [58, Definition 2.1]).

Definition 2.1 A nonempty, open, bounded subset $\Omega$ of $\subset \mathbb{R}^{n}(n \geq 3)$ is called a bounded Lipschitz domain if for any $\mathbf{x} \in \partial \Omega$ there exist some constants $r, h>0$ and a coordinate system in $\mathbb{R}^{n},\left(y_{1}, \ldots, y_{n}\right)=\left(y^{\prime}, y_{n}\right) \in \mathbb{R}^{n-1} \times \mathbb{R}$, which is isometric to the canonical one and has origin at $\mathbf{x}$, along with a Lipschitz function $\varphi: \mathbb{R}^{n-1} \rightarrow \mathbb{R}$, such that the following property holds. If $\mathcal{C}(r, h)$ denotes the open cylinder $\left\{y=\left(y^{\prime}, y_{n}\right) \in \mathbb{R}^{n-1} \times \mathbb{R}:\left|y^{\prime}\right|<r,\left|y_{n}\right|<h\right\} \subseteq \mathbb{R}^{n}$, then

$$
\Omega \cap \mathcal{C}(r, h)=\left\{y=\left(y^{\prime}, y_{n}\right) \in \mathbb{R}^{n-1} \times \mathbb{R}:\left|y^{\prime}\right|<r \text { and } \varphi\left(y^{\prime}\right)<y_{n}<h\right\} .
$$

In view of the Definition 2.1, condition (2.1) implies that $\partial \Omega=\partial \bar{\Omega}$ and the characterization (cf. [58, (2.4)-(2.6)])

$$
\begin{aligned}
& \partial \Omega \cap \mathcal{C}(r, h)=\left\{y=\left(y^{\prime}, y_{n}\right) \in \mathbb{R}^{n-1} \times \mathbb{R}:\left|y^{\prime}\right|<r \text { and } y_{n}=\varphi\left(y^{\prime}\right)\right\}, \\
& \left(\mathbb{R}^{n} \backslash \bar{\Omega}\right) \cap \mathcal{C}(r, h)=\left\{y=\left(y^{\prime}, y_{n}\right) \in \mathbb{R}^{n-1} \times \mathbb{R}:\left|y^{\prime}\right|<r \text { and }-h<y_{n}<\varphi\left(y^{\prime}\right)\right\} .
\end{aligned}
$$

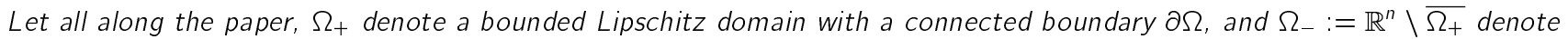
the corresponding exterior domain. Unless stated otherwise, it will be also assumed that $n \geq 3$.

Let $\kappa=\kappa(\partial \Omega)>1$ be a fixed sufficiently large constant. Then the non-tangential maximal operator of an arbitrary function $u: \Omega_{ \pm} \rightarrow \mathbb{R}$ is defined by

$$
M(u)(x):=\left\{\sup |u(y)|: y \in \mathfrak{D}_{ \pm}(x), x \in \partial \Omega\right\}
$$

where

$$
\mathfrak{D}_{ \pm}(x) \equiv \mathfrak{D}_{\kappa ; \pm}(x):=\left\{y \in \Omega_{ \pm}: \operatorname{dist}(x, y)<\kappa \operatorname{dist}(\mathrm{y}, \partial \Omega), x \in \partial \Omega\right\}
$$

are non-tangential approach cones located in $\Omega_{+}$and $\Omega_{-}$, respectively (see, e.g., [61]). Moreover,

$$
u_{\mathrm{nt}}^{ \pm}(x):=\lim _{\mathfrak{D}_{ \pm} \ni y \rightarrow x} u(y)
$$

are the non-tangential limits of $u$ with respect to $\Omega_{ \pm}$at $x \in \partial \Omega$. Note that if $M(u) \in L_{p}(\partial \Omega)$ for one choice of $\kappa$, where $p \in(1, \infty)$, then this property holds for arbitrary choice of $\kappa$ (see, e.g., [47, p. 63]). For the sake of brevity, we use the notation $\mathfrak{D}_{ \pm}(x)$ instead of $\mathfrak{D}_{\kappa ; \pm}(x)$. We often need the property below (cf. [64, page 80], [75, Theorem 1.12]; see also [55, Lemma 2.2])

Lemma 2.2 If $\Omega \subset \mathbb{R}^{n}$ is a Lipschitz domain, then there exists a sequence of $C^{\infty}$ domains $\Omega_{j}$ approximating $\Omega\left(\Omega_{j} \rightarrow \Omega\right.$ as $j \rightarrow \infty)$ in the following sense:

(i) $\bar{\Omega}_{j} \subset \Omega$, and there exists a covering of $\partial \Omega$ with finitely many coordinate cylinders (atlas) that also form a family of coordinate cylinders for $\partial \Omega_{j}$, for each $j$. Moreover, for each such cylinder $\mathcal{C}(r, h)$, if $\varphi$ and $\varphi_{j}$ are the corresponding Lipschitz functions whose graphs describe the boundaries of $\partial \Omega$ and $\partial \Omega_{j}$, respectively, in $\mathcal{C}(r, h)$, then $\left\|\nabla \varphi_{j}\right\|_{L_{\infty}\left(\mathbb{R}^{n-1}\right)} \leq\|\nabla \varphi\|_{L_{\infty}\left(\mathbb{R}^{n-1}\right)}$ and $\nabla \varphi_{j} \rightarrow \nabla \varphi$ pointwise a.e.

(ii) There exist a sequence of Lipschitz diffeomorphisms $\Phi_{j}: \partial \Omega \rightarrow \partial \Omega_{j}$ such that the Lipschitz constants of $\Phi_{j}$, $\Phi_{j}^{-1}$ are uniformly bounded in $j$.

(iii) There is a constant $\kappa>0$ such that for all $j \geq 1$ and all $\mathbf{x} \in \partial \Omega$, we have $\Phi_{j}(\mathbf{x}) \in \mathfrak{D}_{+}(\mathbf{x}) \equiv \mathfrak{D}_{\kappa ; \pm}(x)$, where $\mathfrak{D}_{+}(\mathbf{x}) \equiv$ $\mathfrak{D}_{\kappa ; \pm}(x)$ is the non-tangential approach cone with vertex at $\mathbf{x}$. Moreover,

$$
\begin{aligned}
& \lim _{j \rightarrow \infty}\left|\Phi_{j}(\mathbf{x})-\mathbf{x}\right|=0 \text { uniformly in } \mathbf{x} \in \partial \Omega, \\
& \lim _{j \rightarrow \infty} \boldsymbol{\nu}^{(j)}\left(\Phi_{j}(\mathbf{x})\right)=\boldsymbol{\nu}(\mathbf{x}) \text { for a.e. } \mathbf{x} \in \partial \Omega, \text { and in every space } L_{p}(\partial \Omega), p \in(1, \infty),
\end{aligned}
$$

where $\boldsymbol{\nu}^{(j)}$ is the outward unit normal to $\partial \Omega_{j}$, and $\boldsymbol{\nu}$ is the outward unit normal to $\partial \Omega$

(iv) There exist some positive functions $\omega_{j}: \partial \Omega \rightarrow \mathbb{R}$ (the Jacobian related to $\Phi_{j}, j \in \mathbb{N}$ ) bounded away from zero and infinity uniformly in $j$, such that, for any measurable set $A \subset \partial \Omega, \int_{A} \omega_{j} d \sigma=\int_{\Phi_{j}(A)} d \sigma_{j}$. In addition, $\lim _{j \rightarrow \infty} \omega_{j}=1$ a.e. on $\partial \Omega$ and in every space $L_{p}(\partial \Omega), p \in(1, \infty)$.

Lemma 2.2 implies that the Lipschitz characters of the domains $\Omega_{j}$ are uniformly controlled by the Lipschitz character of $\Omega$. The meaning of Lipschitz character of a Lipschitz domain is given below (cf., e.g., [58, p. 22]).

Definition 2.3 Let $\Omega \subset \mathbb{R}^{n}$ be a Lipschitz domain. Let $\left\{\mathcal{C}_{k}\left(r_{k}, h_{k}\right): 1 \leq k \leq N\right\}$ (with associated Lipschitz functions $\left\{\varphi_{k}\right.$ : $1 \leq k \leq N\}$ ) be an atlas for $\partial \Omega$, i.e., a finite collection of cylinders covering the boundary $\partial \Omega$. Having fixed such an atlas of $\partial \Omega$, the Lipschitz character of $\Omega$ is defined as the set consisting of the numbers $N$, $\max \left\{\left\|\nabla \varphi_{k}\right\|_{L_{\infty}\left(\mathbb{R}^{n-1}\right)}: 1 \leq k \leq N\right\}$, $\min \left\{r_{k}: 1 \leq k \leq N\right\}$, and $\min \left\{h_{k}: 1 \leq k \leq N\right\}$. 


\subsection{Sobolev and Besov spaces and related results}

In this subsection we assume $n \geq 2$. We denote by $\mathcal{D}\left(\mathbb{R}^{n}\right):=C_{\text {comp }}^{\infty}\left(\mathbb{R}^{n}\right)$ the space of infinitely differentiable functions with compact support in $\mathbb{R}^{n}$ and by $\mathcal{D}\left(\mathbb{R}^{n}, \mathbb{R}^{n}\right):=C_{\text {comp }}^{\infty}\left(\mathbb{R}^{n}, \mathbb{R}^{n}\right)$ the space of infinitely differentiable vector-valued functions with compact support in $\mathbb{R}^{n}$. Also, let $\mathcal{E}\left(\Omega_{ \pm}\right):=C^{\infty}\left(\Omega_{ \pm}\right)$denote the space of infinitely differentiable functions and let $\mathcal{D}\left(\Omega_{ \pm}\right):=$ $C_{\text {comp }}^{\infty}\left(\Omega_{ \pm}\right)$be the space of infinitely differentiable functions with compact support in $\Omega_{ \pm}$, equipped with the inductive limit topology. Let ${ }^{\dagger} \mathcal{E}^{\prime}\left(\mathbb{R}^{n}\right)$ and $\mathcal{D}^{\prime}\left(\mathbb{R}^{n}\right)$ be the duals of $\mathcal{E}\left(\mathbb{R}^{n}\right)$ and $\mathcal{D}\left(\mathbb{R}^{n}\right)$, respectively, i.e., the spaces of distributions on $\mathbb{R}^{n}$. The spaces $\mathcal{E}^{\prime}\left(\Omega_{ \pm}\right)$and $\mathcal{D}^{\prime}\left(\Omega_{ \pm}\right)$can be similarly defined.

Let $\mathcal{F}$ denote the Fourier transform defined on the space of tempered distributions to itself, and $\mathcal{F}^{-1}$ be its inverse. For $p \in(1, \infty), L_{p}\left(\mathbb{R}^{n}\right)$ is the Lebesgue space of (equivalence classes of) measurable, $p^{\text {th }}$ integrable functions on $\mathbb{R}^{n}$, and $L_{\infty}\left(\mathbb{R}^{n}\right)$ is the space of (equivalence classes of) essentially bounded measurable functions on $\mathbb{R}^{n}$. For $s \in \mathbb{R}$, the $L_{p}$-based Bessel potential spaces $H_{p}^{s}\left(\mathbb{R}^{n}\right)$ and $H_{p}^{s}\left(\mathbb{R}^{n}, \mathbb{R}^{n}\right)$ are defined by

$$
\begin{aligned}
& H_{p}^{s}\left(\mathbb{R}^{n}\right):=\left\{f:(\mathbb{I}-\triangle)^{\frac{s}{2}} f \in L_{p}\left(\mathbb{R}^{n}\right)\right\}=\left\{f: J^{s} f \in L_{p}\left(\mathbb{R}^{n}\right)\right\}, \\
& H_{p}^{s}\left(\mathbb{R}^{n}, \mathbb{R}^{n}\right):=\left\{\tilde{\mathbf{f}}=\left(f_{1}, f_{2}, \ldots, f_{n}\right): f_{i} \in H_{p}^{s}\left(\mathbb{R}^{n}\right), j=1, \ldots, n\right\},
\end{aligned}
$$

where $J^{s}: \mathcal{S}^{\prime}\left(\mathbb{R}^{n}\right) \rightarrow \mathcal{S}^{\prime}\left(\mathbb{R}^{n}\right)$ is the Bessel potential operator of order $s$ defined by $J^{s} f=\mathcal{F}^{-1}\left(\rho^{s} \mathcal{F} f\right)$ with

$$
\rho(\xi)=\left(1+|\xi|^{2}\right)^{\frac{1}{2}}
$$

(see, e.g., [44, Chapter 3]). Note that $H_{p}^{s}\left(\mathbb{R}^{n}\right)$ is a Banach space with respect to the norm

$$
\|f\|_{H_{p}^{s}\left(\mathbb{R}^{n}\right)}=\left\|J^{s} f\right\|_{L_{p}\left(\mathbb{R}^{n}\right)}=\left\|\mathcal{F}^{-1}\left(\rho^{s} \mathcal{F} f\right)\right\|_{L_{p}\left(\mathbb{R}^{n}\right)} .
$$

For integer $s \geq 0$, the spaces $H_{p}^{s}\left(\mathbb{R}^{n}\right)$ coincide with the Sobolev spaces $W_{p}^{s}\left(\mathbb{R}^{n}\right)$.

The Bessel potential spaces $H_{p}^{s}(\Omega)$ and $\widetilde{H}_{p}^{s}(\Omega)$ are defined by

$$
\begin{aligned}
& H_{p}^{s}(\Omega):=\left\{f \in \mathcal{D}^{\prime}(\Omega): \exists F \in H_{p}^{s}\left(\mathbb{R}^{n}\right) \text { such that }\left.F\right|_{\Omega}=f\right\}, \\
& \widetilde{H}_{p}^{s}(\Omega):=\left\{f \in H_{p}^{s}\left(\mathbb{R}^{n}\right): \operatorname{supp} f \subseteq \bar{\Omega}\right\},
\end{aligned}
$$

and the Bessel potential spaces $H_{p}^{s}\left(\Omega, \mathbb{R}^{n}\right)$ and $\widetilde{H}_{p}^{s}\left(\Omega, \mathbb{R}^{n}\right)$ are defined as the spaces of vector-valued functions (distributions) whose components belong to the spaces $H_{p}^{s}(\Omega)$ and $\widetilde{H}_{p}^{s}(\Omega)$, respectively (see, e.g., [44]). For any $s \in \mathbb{R}, C^{\infty}(\bar{\Omega})$ is dense in $H_{p}^{s}(\Omega)$ and the following duality relations hold (see [29, Proposition 2.9], [24, (1.9)], [63, (4.14)])

$$
\left(H_{p}^{s}(\Omega)\right)^{\prime}=\widetilde{H}_{p^{\prime}}^{-s}(\Omega), \quad H_{p^{\prime}}^{-s}(\Omega)=\left(\widetilde{H}_{p}^{s}(\Omega)\right)^{\prime} .
$$

Here and further on $p, p^{\prime} \in(1, \infty)$ are related as $\frac{1}{p}+\frac{1}{p^{\prime}}=1$.

Replacing $\Omega$ by $\Omega_{-}$in (2.12) and (2.13), one obtains the Bessel potential spaces $H_{p}^{s}\left(\Omega_{-}\right), \widetilde{H}_{p}^{s}\left(\Omega_{-}\right)$.

For $p \in(1, \infty)$ and $s \in(-1,1)$, the boundary Bessel potential space $H_{p}^{s}(\partial \Omega)$ can be defined by using the space $H_{p}^{s}\left(\mathbb{R}^{n-1}\right)$, a partition of unity and pull-pack. In addition, $H_{p^{\prime}}^{-s}(\partial \Omega)=\left(H_{p}^{s}(\partial \Omega)\right)^{\prime}$. We can also equivalently define $H_{p}^{0}(\partial \Omega)=L_{p}(\partial \Omega)$ as the Lebesgue space of measurable, $p^{\text {th }}$ power integrable functions on $\partial \Omega$. In addition, $H_{p}^{1}(\partial \Omega)$ coincides, with equivalent norm, with the Sobolev space

$$
W_{p}^{1}(\partial \Omega):=\left\{f \in L_{p}(\partial \Omega):\|f\|_{W_{p}^{1}(\partial \Omega)}<\infty\right\},\|f\|_{W_{p}^{1}(\partial \Omega)}:=\|f\|_{L_{p}(\partial \Omega)}+\left\|\nabla_{\tan } f\right\|_{L_{p}(\partial \Omega)}
$$

Here the weak tangential gradient of a function $f$ locally integrable on $\partial \Omega$ is $\nabla_{\tan } f:=\left(\nu_{k} \partial_{\tau_{k j}} f\right)_{1<j<n}$, where $\partial_{\tau_{k j}} f$ is defined in the weak form as (cf. e.g., [61, (2.9)]) $\left\langle\partial_{\tau_{k j}} f, \phi\right\rangle_{\partial \Omega}:=-\left\langle f, \partial_{\tau_{k j}} \phi\right\rangle_{\partial \Omega}$ for any $\phi \in \mathcal{D}\left(\mathbb{R}^{n}\right)$ with $\partial_{\tau_{k j}} \phi:=\left.\nu_{k}\left(\partial_{j} \phi\right)\right|_{\partial \Omega}-\left.\nu_{j}\left(\partial_{k} \phi\right)\right|_{\partial \Omega}, j, k=$ $1, \ldots, n$, and $\boldsymbol{\nu}=\left(\nu_{1}, \ldots, \nu_{n}\right)$ is the outward unit normal to $\Omega$, which exists at almost every point on $\partial \Omega$. If $f$ is defined and smooth enough in the vicinity of $\partial \Omega$, then by integrating by parts it is possible to show that the weak definition coincides with the strong one, given by $\partial_{\tau_{k j}} f:=\left.\nu_{k}\left(\partial_{j} f\right)\right|_{\partial \Omega}-\left.\nu_{j}\left(\partial_{k} f\right)\right|_{\partial \Omega}$.

Now, for $s \in \mathbb{R}$ and $p, q \in(1, \infty)$, denote by $B_{p, q}^{s}\left(\mathbb{R}^{n}\right)$ the scale of Besov spaces in $\mathbb{R}^{n}$, see Appendix $A$. Similar to (2.12) and (2.13), the Besov spaces $B_{p, q}^{s}(\Omega)$ and $B_{p, q}^{s}\left(\Omega, \mathbb{R}^{n}\right)$ are defined by

$$
\begin{aligned}
& B_{p, q}^{s}(\Omega):=\left\{f \in \mathcal{D}^{\prime}(\Omega): \exists F \in B_{p, q}^{s}\left(\mathbb{R}^{n}\right) \text { such that }\left.F\right|_{\Omega}=f\right\}, \\
& B_{p, q}^{s}\left(\Omega, \mathbb{R}^{n}\right):=\left\{\mathbf{f}=\left(f_{1}, f_{2}, \ldots, f_{n}\right): f_{i} \in B_{p, q}^{s}(\Omega), j=1, \ldots, n\right\}, \\
& \widetilde{B}_{p, q}^{s}(\Omega):=\left\{f \in B_{p, q}^{s}\left(\mathbb{R}^{n}\right): \operatorname{supp} f \subseteq \bar{\Omega}\right\} .
\end{aligned}
$$


For $s \in[0,1]$ and $p, q \in(1, \infty)$, the Sobolev and Besov spaces $H_{p}^{s}(\partial \Omega)$ and $B_{p, q}^{s}(\partial \Omega)$ on the boundary $\partial \Omega$ can be defined by using the spaces $H_{p}^{s}\left(\mathbb{R}^{n-1}\right)$ and $B_{p, q}^{s}\left(\mathbb{R}^{n-1}\right)$, a partition of unity and the pull-backs of the local parametrization of $\partial \Omega$. In addition, we note that $H_{p}^{-s}(\partial \Omega)=\left(H_{p^{\prime}}^{s}(\partial \Omega)\right)^{\prime}$ and $B_{p, q}^{-s}=\left(B_{p^{\prime}, q^{\prime}}^{s}(\partial \Omega)\right)^{\prime}$, where $p^{\prime}, q^{\prime} \in(1, \infty)$ such that $\frac{1}{p}+\frac{1}{p^{\prime}}=1$ and $\frac{1}{q}+\frac{1}{q^{\prime}}=1$ (for further details about boundary Sobolev and Besov spaces see, e.g., [61, p. 35]).

A useful result for the problems we are going to investigate in this paper is the following trace lemma (see [30, Chapter VIII, Theorems 1,2], [29, Theorem 3.1] and also [18, Lemma 3.6] for the case $p=2$ and a discussion on the critical smoothness index $s=1$ ).

Lemma 2.4 Assume that $\Omega \subset \mathbb{R}^{n}$ is a bounded Lipschitz domain with connected boundary $\partial \Omega$ and let $\Omega_{-}:=\mathbb{R}^{n} \backslash \bar{\Omega}$ be the corresponding exterior domain. Let $p, q \in(1, \infty)$ and $s \in(0,1)$. Then there exist linear and continuous Gagliardo trace operators $\gamma_{ \pm}: H_{p}^{s+\frac{1}{p}}\left(\Omega_{ \pm}\right) \rightarrow B_{p, p}^{s}(\partial \Omega)$ and $\gamma_{ \pm}: B_{p, q}^{s+\frac{1}{p}}\left(\Omega_{ \pm}\right) \rightarrow B_{p, q}^{s}(\partial \Omega)$, respectively, such that $\gamma_{ \pm} f=\left.f\right|_{\partial \Omega}$ for any $f \in C^{\infty}\left(\bar{\Omega}_{ \pm}\right)$. These operators are surjective and have (non-unique) linear and continuous right inverse operators $\gamma_{ \pm}^{-1}: B_{p, p}^{s}(\partial \Omega) \rightarrow H_{p}^{s+\frac{1}{p}}\left(\Omega_{ \pm}\right)$and $\gamma_{ \pm}^{-1}: B_{p, q}^{s}(\partial \Omega) \rightarrow B_{p, q}^{s+\frac{1}{p}}\left(\Omega_{ \pm}\right)$, respectively.

Lemma 2.4 holds also for vector-valued and matrix-valued functions $f$. If $f$ is such that $\gamma_{+} f=\gamma_{-} f$, we will often write $\gamma f$.

We have the following trace equivalence assertion.

Theorem 2.5 Assume that $\Omega \subset \mathbb{R}^{n}$ is a bounded Lipschitz domain with connected boundary $\partial \Omega$ and let $\Omega_{-}:=\mathbb{R}^{n} \backslash \bar{\Omega}$ be the corresponding exterior domain. Let $p, q \in(1, \infty)$, and let $u \in B_{p, q}^{s+\frac{1}{p}}\left(\Omega_{ \pm}\right)$or $u \in H_{p}^{s+\frac{1}{p}}\left(\Omega_{ \pm}\right)$for some $s>0$. Then the Gagliardo trace $\gamma_{+} u$ is well defined on $\partial \Omega$ and, moreover,

(i) if the pointwise non-tangential trace $u_{\mathrm{nt}}^{ \pm}$exists a.e. on $\partial \Omega$, then $u_{\mathrm{nt}}^{ \pm}=\gamma_{ \pm} u$;

(ii) if the pointwise non-tangential trace $u_{\mathrm{nt}}^{ \pm}$exists a.e. on $\partial \Omega$ and $s \in(0,1)$ then $u_{\mathrm{nt}}^{ \pm}=\gamma_{ \pm} u \in B_{p, q}^{s}(\partial \Omega)$;

(iii) if $u_{\mathrm{nt}}^{ \pm} \in H_{p}^{s}(\partial \Omega)$ for some $s \in(0,1]$, then $\gamma_{ \pm} u \in H_{p}^{s}(\partial \Omega)$ as well.

Proof. Item (i) for $0<s<1$ is implied by Theorem 8.7(iii) in [6], while for $s \geq 1$ the equality $\gamma_{ \pm} u=u_{\mathrm{nt}}^{ \pm}$still applies by an imbedding argument. Item (ii) and (iii) follow from item (i) and the well known imbedding $\gamma_{ \pm} u \in B_{p, q}^{s}(\partial \Omega)$ for $s \in(0,1)$.

Further on, $\langle\cdot, \cdot\rangle_{\Omega^{\prime}}$ will denote the dual form between corresponding dual spaces defined on a set $\Omega^{\prime}$. For further details about Sobolev, Bessel potential and Besov spaces, we refer the reader to, e.g., [1, 27, 44, 72, 73].

\subsection{The Brinkman system and conormal derivatives in Bessel-potential and Besov spaces}

In this subsection we also assume $n \geq 2$. For a couple $(\mathbf{u}, \pi)$, and a real number $\alpha \geq 0$, let us consider the linear Brinkman system (in the incompressible case)

$$
\mathcal{L}_{\alpha}(\mathbf{u}, \pi)=\mathbf{f}, \operatorname{div} \mathbf{u}=0
$$

where the Brinkman operator is defined as

$$
\mathcal{L}_{\alpha}(\mathbf{u}, \pi):=\triangle \mathbf{u}-\alpha \mathbf{u}-\nabla \pi \text {. }
$$

When $\alpha=0$, the Brinkman operator becomes the Stokes operator.

Now, for $(\mathbf{u}, \pi) \in C^{1}\left(\bar{\Omega}_{ \pm}, \mathbb{R}^{n}\right) \times C^{0}\left(\bar{\Omega}_{ \pm}\right)$, such that div $\mathbf{u}=0$ in $\Omega_{ \pm}$, we define the classical conormal derivatives (tractions) for the Brinkman (or the Stokes) system, $\mathbf{t}_{\alpha}^{\mathrm{c}}(\mathbf{u}, \pi)$, by using the well-known formula

$$
\mathbf{t}^{\mathrm{c} \pm}(\mathbf{u}, \pi):=\left(\gamma_{ \pm} \boldsymbol{\sigma}(\mathbf{u}, \pi)\right) \boldsymbol{\nu}
$$

where

$$
\boldsymbol{\sigma}(\mathbf{u}, \pi):=-\pi \mathbb{I}+2 \mathbb{E}(\mathbf{u})
$$

is the stress tensor, $\mathbb{E}(\mathbf{u})$ is the strain rate tensor (symmetric part of $\nabla \mathbf{u}$ ), and $\boldsymbol{\nu}=\boldsymbol{\nu}^{+}$is the outward unit normal to $\Omega_{+}$, defined a.e. on $\partial \Omega$. Then for any function $\boldsymbol{\varphi} \in \mathcal{D}\left(\mathbb{R}^{n}, \mathbb{R}^{n}\right)$ we obtain by integrating by parts the first Green identity,

$$
\pm\left\langle\mathbf{t}^{\mathrm{ct}}(\mathbf{u}, \pi), \boldsymbol{\varphi}\right\rangle_{\partial \Omega}=2\langle\mathbb{E}(\mathbf{u}), \mathbb{E}(\boldsymbol{\varphi})\rangle_{\Omega_{ \pm}}+\alpha\langle\mathbf{u}, \boldsymbol{\varphi}\rangle_{\Omega_{ \pm}}-\langle\pi, \operatorname{div} \boldsymbol{\varphi}\rangle_{\Omega_{ \pm}}+\left\langle\mathcal{L}_{\alpha}(\mathbf{u}, \pi), \boldsymbol{\varphi}\right\rangle_{\Omega_{ \pm}} .
$$

If the non-tangential traces of the stress tensor, $\boldsymbol{\sigma}_{\mathrm{nt}}^{ \pm}(\mathbf{u}, \pi)$ and the normal vector $\boldsymbol{\nu}$ exist at a boundary point, then the non-tangential conormal derivatives are defined at this point as

$$
\mathbf{t}_{\mathrm{nt}}^{ \pm}(\mathbf{u}, \pi):=\boldsymbol{\sigma}_{\mathrm{nt}}^{ \pm} \boldsymbol{\nu}
$$

For $s \in \mathbb{R}$ and $p, q \in(1, \infty)$, we consider the spaces

$$
\begin{aligned}
& H_{p ; \operatorname{div}}^{s}\left(\Omega_{ \pm}, \mathbb{R}^{n}\right)=\left\{\mathbf{u}_{ \pm} \in H_{p}^{s}\left(\Omega_{ \pm}, \mathbb{R}^{n}\right): \operatorname{div} \mathbf{u}=0 \text { in } \Omega_{ \pm}\right\} \\
& B_{p, q, \operatorname{div}}^{s}\left(\Omega_{ \pm}, \mathbb{R}^{n}\right):=\left\{\mathbf{u}_{ \pm} \in B_{p, q}^{s}\left(\Omega_{ \pm}, \mathbb{R}^{n}\right): \operatorname{div} \mathbf{u}=0 \text { in } \Omega_{ \pm}\right\} .
\end{aligned}
$$

We need also the following spaces (cf. [51, Definition 3.3]). 
Definition 2.6 Let $\Omega$ be a Lipschitz domain (bounded or unbounded). For $s \in \mathbb{R}, p, q \in(1, \infty)$ and $t \geq-1 / p^{\prime}$, let us consider the following spaces equipped with the corresponding graphic norms:

$$
\begin{aligned}
& \mathfrak{H}_{p, \operatorname{div}}^{s+\frac{1}{p}, t}\left(\Omega, \mathcal{L}_{\alpha}\right):=\left\{(\mathbf{u}, \pi) \in H_{p}^{s+\frac{1}{p}}\left(\Omega, \mathbb{R}^{n}\right) \times H_{p}^{s+\frac{1}{p}-1}(\Omega): \mathcal{L}_{\alpha}(\mathbf{u}, \pi)=\left.\tilde{\mathbf{f}}\right|_{\Omega}, \tilde{\mathbf{f}} \in \widetilde{H}_{p}^{t}\left(\Omega, \mathbb{R}^{n}\right) \text { and } \operatorname{div} \mathbf{u}=0 \text { in } \Omega\right\}, \\
& \|(\mathbf{u}, \pi)\|_{\mathfrak{H}_{p, \mathrm{div}}^{s+\frac{1}{, t}\left(\Omega, \mathcal{L}_{\alpha}\right)}}^{2}:=\|\mathbf{u}\|_{H_{p}^{s+\frac{1}{p}}\left(\Omega, \mathbb{R}^{n}\right)}^{2}+\|\pi\|_{H_{p}^{s+\frac{1}{p}-1}(\Omega)}^{2}+\|\tilde{\mathbf{f}}\|_{\widetilde{H}_{p}^{t}\left(\Omega, \mathbb{R}^{n}\right)}^{2}, \\
& \mathfrak{B}_{p, q, \operatorname{div}}^{s+\frac{1}{,}, t}\left(\Omega, \mathcal{L}_{\alpha}\right):=\left\{(\mathbf{u}, \pi) \in B_{p, q}^{s+\frac{1}{p}}\left(\Omega, \mathbb{R}^{n}\right) \times B_{p, q}^{s+\frac{1}{p}-1}(\Omega): \mathcal{L}_{\alpha}(\mathbf{u}, \pi)=\left.\tilde{\mathbf{f}}\right|_{\Omega}, \tilde{\mathbf{f}} \in \widetilde{B}_{p, q}^{t}\left(\Omega, \mathbb{R}^{n}\right) \text { and } \operatorname{div} \mathbf{u}=0 \text { in } \Omega\right\} \text {, } \\
& \|(\mathbf{u}, \pi)\|_{\mathfrak{B}_{p, q, \mathrm{div}}^{s+\frac{1}{p}, t}\left(\Omega, \mathcal{L}_{\alpha}\right)}^{2}:=\|\mathbf{u}\|_{B_{p, q}^{s+\frac{1}{p}}\left(\Omega, \mathbb{R}^{n}\right)}^{2}+\|\pi\|_{B_{p, q}^{s+\frac{1}{p}-1}(\Omega)}^{2}+\|\tilde{\mathbf{f}}\|_{\widetilde{B}_{p, q}^{t}\left(\Omega, \mathbb{R}^{n}\right)}^{2},
\end{aligned}
$$

where $\mathcal{L}_{\alpha}(\mathbf{u}, \pi)$ is defined in (2.20).

If $t_{1}>t_{2}$, the following continuous embeddings hold, $\mathfrak{H}_{p, \text { div }}^{s+\frac{1}{p}, t_{1}}\left(\Omega, \mathcal{L}_{\alpha}\right) \hookrightarrow \mathfrak{H}_{p, \text { div }}^{s+\frac{1}{,}, t_{2}}\left(\Omega, \mathcal{L}_{\alpha}\right), \mathfrak{B}_{p, q \text {, div }}^{s+\frac{1}{p}, t_{1}}\left(\Omega, \mathcal{L}_{\alpha}\right) \hookrightarrow \mathfrak{B}_{p, q, \text { div }}^{s+\frac{1}{p}, t_{2}}\left(\Omega, \mathcal{L}_{\alpha}\right)$.

Let $\mathcal{D}_{\text {div }}\left(\bar{\Omega}, \mathbb{R}^{n}\right):=\left\{\mathbf{v} \in \mathcal{D}\left(\bar{\Omega}, \mathbb{R}^{n}\right): \operatorname{div} \mathbf{v}=0\right.$ in $\left.\Omega\right\}$. Similar to [52, Theorem 6.9], one can prove the following assertion.

Theorem 2.7 If $\Omega$ is a Lipschitz domain (bounded or unbounded) or $\Omega=\mathbb{R}^{n}, \alpha \geq 0, p, q \in(1, \infty), s \in \mathbb{R}$ and $t>-\frac{1}{p^{\prime}}$, then $\mathcal{D}_{\text {div }}\left(\bar{\Omega}, \mathbb{R}^{n}\right) \times \mathcal{D}(\bar{\Omega})$ is dense in $\mathfrak{H}_{p}^{s+\frac{1}{p}, t}\left(\Omega, \mathcal{L}_{\alpha}\right)$ and in $\mathfrak{B}_{p, q}^{s+\frac{1}{p}, t}\left(\Omega, \mathcal{L}_{\alpha}\right)$.

Let $p, q \in(1, \infty)$. Let $\stackrel{\circ}{E}_{ \pm}$be the operator of extension of functions defined on $\Omega_{ \pm}$by zero on $\mathbb{R}^{n} \backslash \Omega_{ \pm}$. Following the proof of Theorem 2.16 in [51], let us define the operator $\widetilde{E}_{ \pm}$on $H_{p}^{t}\left(\Omega_{ \pm}\right)$and $B_{p, q}^{t}\left(\Omega_{ \pm}\right)$as $\widetilde{E}_{ \pm}:=\dot{E}_{ \pm}$for $0 \leq t<\frac{1}{p}$, and as

$$
\left\langle\widetilde{E}_{ \pm} h, v\right\rangle_{\Omega_{ \pm}}:=\left\langle h, \widetilde{E}_{ \pm} v\right\rangle_{\Omega_{ \pm}}=\left\langle h, \stackrel{\circ}{E}_{ \pm} v\right\rangle_{\Omega_{ \pm}} \text {, when }-\frac{1}{p^{\prime}}<t<0,
$$

for all $h \in H_{p}^{t}\left(\Omega_{ \pm}\right), v \in H_{p^{\prime}}^{-t}\left(\Omega_{ \pm}\right)$, or for all $h \in B_{p, q}^{t}\left(\Omega_{ \pm}\right), v \in B_{p^{\prime}, q^{\prime}}^{-t}\left(\Omega_{ \pm}\right)$, respectively. Then, for $-1 / p^{\prime}<t<1 / p$, evidently

$$
\widetilde{E}_{ \pm}: H_{p}^{t}\left(\Omega_{ \pm}\right) \rightarrow \widetilde{H}_{p}^{t}\left(\Omega_{ \pm}\right), \quad \widetilde{E}_{ \pm}: B_{p, q}^{t}\left(\Omega_{ \pm}\right) \rightarrow \widetilde{B}_{p, q}^{t}\left(\Omega_{ \pm}\right)
$$

are bounded linear extension operators. Similar definition and properties hold also for vector fields.

Analogously to the corresponding definition for Petrovskii-elliptic systems in [51, Definition 3.6], we can introduce an operator $\tilde{\mathcal{L}}_{\alpha}$ as follows.

Definition 2.8 Let $\Omega$ be a Lipschitz domain (bounded or unbounded), $p, q \in(1, \infty), s \in \mathbb{R}, t \geq-1 / p^{\prime}$. The operator $\tilde{\mathcal{L}}_{\alpha}$ mapping

(i) functions $(\mathbf{u}, \pi) \in \mathfrak{H}_{p, \text { div }}^{s+\frac{1}{p}, t}\left(\Omega, \mathcal{L}_{\alpha}\right)$ to the extension of the distribution $\mathcal{L}_{\alpha}(\mathbf{u}, \pi) \in H_{p}^{t}\left(\Omega, \mathbb{R}^{n}\right)$ to $\widetilde{H}_{p}^{t}\left(\Omega, \mathbb{R}^{n}\right)$

or

(ii) functions $(\mathbf{u}, \pi) \in \mathfrak{B}_{p, q, \mathrm{div}}^{s+\frac{1}{,}, t}\left(\Omega ; \mathcal{L}_{\alpha}\right)$ to the extension of the distribution $\mathcal{L}_{\alpha}(\mathbf{u}, \pi) \in B_{p, q}^{t}\left(\Omega, \mathbb{R}^{n}\right)$ to $\widetilde{B}_{p, q}^{t}\left(\Omega, \mathbb{R}^{n}\right)$, will be called the canonical extension of the operator $\mathcal{L}_{\alpha}$

Remark 2.9 Similar to the paragraph following Definition 3.3 in [51], one can prove that the canonical extensions mentioned in Definition 2.8 exist and are unique. If $p, q \in(1, \infty), s \in \mathbb{R}, t \geq-1 / p^{\prime}$, then

$$
\left\|\tilde{\mathcal{L}}_{\alpha}(\mathbf{u}, \pi)\right\|_{\widetilde{H}_{p}^{t}\left(\Omega, \mathbb{R}^{n}\right)} \leq\|(\mathbf{u}, \pi)\|_{\mathfrak{H}_{p, \text { div }}^{s+\frac{1}{p}, t}\left(\Omega, \mathcal{L}_{\alpha}\right)} \text { and }\left\|\tilde{\mathcal{L}}_{\alpha}(\mathbf{u}, \pi)\right\|_{\widetilde{B}_{p, q}^{t}\left(\Omega, \mathbb{R}^{n}\right)} \leq\|(\mathbf{u}, \pi)\|_{\mathfrak{B}_{p, q, \text { div }}^{s+\frac{1}{p}, t}\left(\Omega, \mathcal{L}_{\alpha}\right)}
$$

by definition of the spaces $\mathfrak{H}_{p, \text { div }}^{s+\frac{1}{p}, t}\left(\Omega, \mathcal{L}_{\alpha}\right)$ and $\mathfrak{B}_{p, q \text {,div }}^{s+\frac{1}{p}, t}\left(\Omega, \mathcal{L}_{\alpha}\right)$. Hence the linear operators $\tilde{\mathcal{L}}_{\alpha}: \mathfrak{H}_{p, \text { div }}^{s+\frac{1}{,}, t}\left(\Omega, \mathcal{L}_{\alpha}\right) \rightarrow \widetilde{H}_{p}^{t}\left(\Omega, \mathbb{R}^{n}\right)$ and $\tilde{\mathcal{L}}_{\alpha}: \mathfrak{B}_{p, q, \text { div }}^{s+\frac{1}{p}, t}\left(\Omega, \mathcal{L}_{\alpha}\right) \rightarrow \widetilde{B}_{p, q}^{t}\left(\Omega, \mathbb{R}^{n}\right)$ are continuous. Moreover, if $-1 / p^{\prime}<t<1 / p$, and $\Omega$ is a Lipschitz domain (bounded or unbounded), then we have the representation $\tilde{\mathcal{L}}_{\alpha}:=\widetilde{E}^{+} \mathcal{L}_{\alpha}$, or $\tilde{\mathcal{L}}_{\alpha}:=\widetilde{E}^{-} \mathcal{L}_{\alpha}$, respectively, cf. [51, Remark 3.7].

Formula (2.23) suggests the following definition of the canonical conormal derivative in the setting of Besov spaces, cf., [18, Lemma 3.2], [36, Lemma 2.2], [51, Definition 3.8, Theorem 3.9], [52, Definition 6.5, Theorem 6.6], [61, Proposition 10.2.1]).

Definition 2.10 Let $\alpha \geq 0, s \in(0,1), p, q \in(1, \infty)$. Then the canonical conormal derivative operators $\mathbf{t}_{\alpha}^{ \pm}$are defined on any $(\mathbf{u}, \pi) \in \mathfrak{H}_{p, \text { div }}^{s+\frac{1}{p^{\prime}},-\frac{1}{p^{\prime}}}\left(\Omega_{ \pm}, \mathcal{L}_{\alpha}\right)$, or $(\mathbf{u}, \pi) \in \mathfrak{B}_{p, q \text {, div }}^{s+\frac{1}{p},-\frac{1}{p^{\prime}}}\left(\Omega_{ \pm}, \mathcal{L}_{\alpha}\right)$, in the weak sense, by the formula

$$
\begin{array}{r} 
\pm\left\langle\mathbf{t}_{\alpha}^{ \pm}(\mathbf{u}, \pi), \boldsymbol{\varphi}\right\rangle_{\partial \Omega_{ \pm}}:=2\left\langle\widetilde{E}_{ \pm} \mathbb{E}(\mathbf{u}), \mathbb{E}\left(\gamma_{ \pm}^{-1} \boldsymbol{\varphi}\right)\right\rangle_{\Omega_{ \pm}}+\alpha\left\langle\widetilde{E}_{ \pm} \mathbf{u}, \gamma_{ \pm}^{-1} \boldsymbol{\varphi}\right\rangle_{\Omega_{ \pm}}-\left\langle\widetilde{E}_{ \pm} \pi, \operatorname{div}\left(\gamma_{ \pm}^{-1} \boldsymbol{\varphi}\right)\right\rangle_{\Omega_{ \pm}}+\left\langle\tilde{\mathcal{L}}_{\alpha}(\mathbf{u}, \pi), \gamma_{ \pm}^{-1} \boldsymbol{\varphi}\right\rangle_{\Omega_{ \pm}} \\
\forall \boldsymbol{\varphi} \in B_{p^{\prime}, p^{\prime}}^{1-s}\left(\partial \Omega, \mathbb{R}^{n}\right), \quad \text { or } \forall \boldsymbol{\varphi} \in B_{p^{\prime}, q^{\prime}}^{1-s}\left(\partial \Omega, \mathbb{R}^{n}\right), \text { respectively. }
\end{array}
$$


Note that the canonical conormal derivative operators introduced in Definition 2.10 are different from the generalized conormal derivative operator, cf. [37, Lemma 2.2], [51, Definition 3.1, Theorem 3.2], [52, Definition 5.2, Theorem 5.3]. Similar to [51, Theorem 3.9], one can prove the following assertion.

Lemma 2.11 Under the hypothesis of Definition 2.10, the canonical conormal derivative operators

$$
\mathbf{t}_{\alpha}^{ \pm}: \mathfrak{H}_{p, \text { div }}^{s+\frac{1}{p},-\frac{1}{p^{\prime}}}\left(\Omega_{ \pm}, \mathcal{L}_{\alpha}\right) \rightarrow B_{p, p}^{s-1}\left(\partial \Omega, \mathbb{R}^{n}\right), \mathbf{t}_{\alpha}^{ \pm}: \mathfrak{B}_{p, q, \mathrm{div}}^{s+\frac{1}{p},-\frac{1}{p^{\prime}}}\left(\Omega_{ \pm}, \mathcal{L}_{\alpha}\right) \rightarrow B_{p, q}^{s-1}\left(\partial \Omega, \mathbb{R}^{n}\right)
$$

are linear, bounded and independent of the choice of the operators $\gamma_{ \pm}^{-1}$. In addition, the following first Green identity holds

$$
\pm\left\langle\mathbf{t}_{\alpha}^{ \pm}(\mathbf{u}, \pi), \gamma_{+} \mathbf{w}\right\rangle_{\partial \Omega}=2\left\langle\widetilde{E}_{ \pm} \mathbb{E}(\mathbf{u}), \mathbb{E}(\mathbf{w})\right\rangle_{\Omega_{ \pm}}+\alpha\left\langle\widetilde{E}_{ \pm} \mathbf{u}, \mathbf{w}\right\rangle_{\Omega_{ \pm}}-\left\langle\widetilde{E}_{ \pm} \pi, \operatorname{div} \mathbf{w}\right\rangle_{\Omega_{ \pm}}+\left\langle\tilde{\mathcal{L}}_{\alpha}(\mathbf{u}, \pi), \mathbf{w}\right\rangle_{\Omega_{ \pm}}
$$

for all $(\mathbf{u}, \pi) \in \mathfrak{H}_{p, \text { div }}^{s+\frac{1}{p},-\frac{1}{p^{\prime}}}\left(\Omega_{ \pm}, \mathcal{L}_{\alpha}\right), \mathbf{w} \in H_{p^{\prime}}^{1+\frac{1}{p^{\prime}}-s}\left(\Omega_{ \pm}, \mathbb{R}^{n}\right)$ and all $(\mathbf{u}, \pi) \in \mathfrak{B}_{p, q \text {,div }}^{s+\frac{1}{p^{\prime}}, \frac{1}{p^{\prime}}}\left(\Omega_{ \pm}, \mathcal{L}_{\alpha}\right)$, w $\in B_{p^{\prime}, q^{\prime}}^{1+\frac{1}{p^{\prime}}-s}\left(\Omega_{ \pm}, \mathbb{R}^{n}\right)$, and the following second Green identity holds

$$
\pm\left(\left\langle\mathbf{t}_{\alpha}^{ \pm}(\mathbf{u}, \pi), \gamma_{+} \mathbf{v}\right\rangle_{\partial \Omega}-\left\langle\mathbf{t}_{\alpha}^{ \pm}(\mathbf{v}, q), \gamma_{+} \mathbf{u}\right\rangle_{\partial \Omega}\right)=\left\langle\tilde{\mathcal{L}}_{\alpha}(\mathbf{u}, \pi), \mathbf{v}\right\rangle_{\Omega_{ \pm}}-\left\langle\tilde{\mathcal{L}}_{\alpha}(\mathbf{v}, q), \mathbf{u}\right\rangle_{\Omega_{ \pm}}
$$

for all $(\mathbf{u}, \pi) \in \mathfrak{H}_{p, \text { div }}^{s+\frac{1}{p},-\frac{1}{p^{\prime}}}\left(\Omega_{ \pm}, \mathcal{L}_{\alpha}\right),(\mathbf{v}, q) \in \mathfrak{H}_{p^{\prime}, \text { div }}^{1+\frac{1}{p^{\prime}}-s,-\frac{1}{p}}\left(\Omega_{ \pm}, \mathbb{R}^{n}\right)$ and all $(\mathbf{u}, \pi) \in \mathfrak{B}_{p, q \text {,div }}^{s+\frac{1}{p},-\frac{1}{p^{\prime}}}\left(\Omega_{ \pm}, \mathcal{L}_{\alpha}\right),(\mathbf{v}, q) \in \mathfrak{B}_{p^{\prime}, q^{\prime}}^{1+\frac{1}{p^{\prime}}-s,-\frac{1}{p}}\left(\Omega_{ \pm}, \mathbb{R}^{n}\right)$

Remark 2.12 Similar to [32, Remark 2.6], we note that by exploiting arguments analogous to those of the proof of Theorem 3.10 and the paragraph following it in [51], one can see that the canonical conormal derivatives on $\partial \Omega$ can be equivalently defined as $\mathbf{t}_{\alpha}^{ \pm}(\mathbf{u}, \pi)=r_{\partial \Omega} \mathbf{t}_{\alpha}^{\prime \pm}(\mathbf{u}, \pi)$. Here $\mathbf{t}_{\alpha}^{\prime \pm}(\mathbf{u}, \pi)$ is defined by the dual form like (2.27) but only on Lipschitz subsets $\Omega_{ \pm}^{\prime} \subset \Omega_{ \pm}$ such that $\partial \Omega \subset \partial \Omega_{ \pm}^{\prime}$ and closure of $\Omega_{ \pm} \backslash \overline{\Omega_{ \pm}^{\prime}}$ coincides with $\Omega_{ \pm} \backslash \Omega_{ \pm}^{\prime}$ (i.e., $\Omega_{ \pm}^{\prime}$ are some layers near the boundary $\partial \Omega$ ). Moreover, such a definition is well applicable to the functions $(\mathbf{u}, \pi)$ from $\mathfrak{H}_{p, \text { div }}^{s+\frac{1}{p},-\frac{1}{p^{\prime}}}\left(\Omega_{ \pm}^{\prime}, \mathcal{L}_{\alpha}\right)$ or $\mathfrak{B}_{p, q \text {, div }}^{s+\frac{1}{p},-\frac{1}{p^{\prime}}}\left(\Omega_{ \pm}^{\prime}, \mathcal{L}_{\alpha}\right)$ that are not obliged to belong to $\mathfrak{H}_{p \text {, div }}^{s+\frac{1}{p},-\frac{1}{p^{\prime}}}\left(\Omega_{ \pm}, \mathcal{L}_{\alpha}\right)$ or $\mathfrak{B}_{p, q \text {, div }}^{s+\frac{1}{p},-\frac{1}{p^{\prime}}}\left(\Omega_{ \pm}, \mathcal{L}_{\alpha}\right)$, respectively. It is particularly useful for the functions (u, $\left.\pi\right)$ that belong to $\mathfrak{H}_{p, \text { div }}^{s+\frac{1}{p},-\frac{1}{p^{\prime}}}\left(\bar{\Omega}_{-}, \mathcal{L}_{\alpha}\right)$ or $\mathfrak{B}_{p, q, \text { div }}^{s+\frac{1}{p^{\prime}}, \frac{1}{\prime}}\left(\bar{\Omega}_{-}, \mathcal{L}_{\alpha}\right)$ only locally.

Now we prove the equivalence between canonical and non-tangential conormal derivatives (as well as classical conormal derivative, when appropriate).

Theorem 2.13 Let $n \geq 2, \alpha \geq 0$, and $p, q \in(1, \infty)$.

(i) Let $s>1$ and $(\mathbf{u}, \pi) \in B_{p, q \text {,div }}^{s+\frac{1}{p}}\left(\Omega_{ \pm}, \mathbb{R}^{n}\right) \times B_{p, q}^{s-1+\frac{1}{p}}\left(\Omega_{ \pm}\right)$or $(\mathbf{u}, \pi) \in H_{p, \text { div }}^{s+\frac{1}{p}}\left(\Omega_{ \pm}, \mathbb{R}^{n}\right) \times H_{q}^{s-1+\frac{1}{p}}\left(\Omega_{ \pm}\right)$. Then the classical conormal derivative $\mathbf{t}^{\mathrm{c} \pm}(\mathbf{u}, \pi)$ and the canonical conormal derivative $\mathbf{t}_{\alpha}^{ \pm}(\mathbf{u}, \pi)$ are well defined and $\mathbf{t}_{\alpha}^{ \pm}(\mathbf{u}, \pi)=\mathbf{t}^{\mathrm{c} \pm}(\mathbf{u}, \pi) \in$ $L_{p}\left(\partial \Omega, \mathbb{R}^{n}\right)$.

If, moreover, the non-tangential trace of the stress tensor, $\boldsymbol{\sigma}_{\mathrm{nt}}^{ \pm}(\mathbf{u}, \pi)$, exists a.e. on $\partial \Omega$, then the non-tangential conormal derivative, defined by (2.24), also exists a.e. on $\partial \Omega$ and $\mathbf{t}_{\mathrm{nt}}^{ \pm}(\mathbf{u}, \pi)=\mathbf{t}_{\alpha}^{ \pm}(\mathbf{u}, \pi)=\mathbf{t}^{\mathrm{c}}(\mathbf{u}, \pi) \in L_{p}\left(\partial \Omega, \mathbb{R}^{n}\right)$.

(ii) Let $0<s \leq 1,(\mathbf{u}, \pi) \in \mathfrak{B}_{p, q \text {,div }}^{s+\frac{1}{p}, t}\left(\Omega_{ \pm}, \mathcal{L}_{\alpha}\right)$ or $(\mathbf{u}, \pi) \in \mathfrak{H}_{p, \text { div }}^{s+\frac{1}{p}, t}\left(\Omega_{ \pm}, \mathcal{L}_{\alpha}\right)$, for some $t>-\frac{1}{p^{\prime}}$. Let also assume that the nontangential maximal function $M(\boldsymbol{\sigma}(\mathbf{u}, \pi))$ and the non-tangential trace of the stress tensor, $\boldsymbol{\sigma}_{\mathrm{nt}}^{ \pm}(\mathbf{u}, \pi)$, exist and are finite a.e. on $\partial \Omega$ and belong to the space $L_{p}\left(\partial \Omega, \mathbb{R}^{n \times n}\right)$. Then $\mathbf{t}_{\alpha}^{ \pm}(\mathbf{u}, \pi)=\mathbf{t}_{\mathrm{nt}}^{ \pm}(\mathbf{u}, \pi) \in L_{p}\left(\partial \Omega, \mathbb{R}^{n}\right)$.

Proof. We will give a proof in the case of a bounded domain $\Omega_{+}$and the Besov spaces. For an unbounded domain $\Omega_{-}$and the Bessel potential spaces the arguments are the same.

(i) Let $(\mathbf{u}, \pi) \in B_{p, q \text {, div }}^{s+\frac{1}{p}}\left(\Omega_{+}, \mathbb{R}^{n}\right) \times B_{p, q}^{s-1+\frac{1}{p}}\left(\Omega_{+}\right)$for some $p, q \in(1, \infty)$ and $s>1$. Evidently, the stress tensor $\boldsymbol{\sigma}(\mathbf{u}, \pi)$ belongs to $B_{p, q}^{s-1+\frac{1}{p}}\left(\Omega, \mathbb{R}^{n \times n}\right)$, which for $1<s<2$ implies that $\gamma_{-} \boldsymbol{\sigma}(\mathbf{u}, \pi) \in B_{p, q}^{s-1}\left(\partial \Omega, \mathbb{R}^{n \times n}\right) \subset L_{p}\left(\partial \Omega, \mathbb{R}^{n \times n}\right)$. Taking into account that the unit normal vector to the boundary, $\boldsymbol{\nu}$, belongs to $L_{\infty}\left(\partial \Omega, \mathbb{R}^{n}\right)$, we obtain by $(2.21)$ that $\mathbf{t}^{\mathrm{c}}(\mathbf{u}, \pi) \in L_{p}\left(\partial \Omega, \mathbb{R}^{n}\right)$.

On the other hand, the inclusion $(\mathbf{u}, \pi) \in B_{p, q \text {, div }}^{s+\frac{1}{p}}\left(\Omega_{+}\right) \times B_{p, q}^{s-1+\frac{1}{p}}\left(\Omega_{+}\right)$for $p, q \in(1, \infty)$ and $s>1$ implies that (u, $\pi$ ) $\in$ $\mathfrak{B}_{p, q, \text { div }}^{s+\frac{1}{,}, t}\left(\Omega_{+}, \mathcal{L}_{\alpha}\right)$ for $t \in\left(-1 / p^{\prime}, s-1-1 / p^{\prime}\right)$ and thus the canonical conormal derivative $\mathbf{t}_{\alpha}^{+}(\mathbf{u}, \pi)$ is well defined and belongs to $B_{p, q}^{s^{\prime}-1}\left(\partial \Omega, \mathbb{R}^{n}\right)$ for any $s^{\prime} \in(0,1)$. For $1<s<2$, the proof that $\mathbf{t}_{\alpha}^{+}(\mathbf{u}, \pi)=\mathbf{t}_{\alpha}^{\mathrm{c}+}(\mathbf{u}, \pi) \in L_{p}\left(\partial \Omega, \mathbb{R}^{n}\right)$ is similar to $[51$, Corollary 3.14] (with evident modification to $L_{p}$-based spaces), while for $s \geq 2$ the relation $\mathbf{t}_{\alpha}^{+}(\mathbf{u}, \pi)=\mathbf{t}^{\mathrm{c}}(\mathbf{u}, \pi) \in L_{p}\left(\partial \Omega, \mathbb{R}^{n}\right)$ still stays by imbedding.

If, in addition, the non-tangential trace of the stress, $\boldsymbol{\sigma}_{\mathrm{nt}}^{+}(\mathbf{u}, \pi)$, exists a.e. on $\partial \Omega$, then $\boldsymbol{\sigma}_{\mathrm{nt}}^{+}(\mathbf{u}, \pi)=\gamma^{+} \boldsymbol{\sigma}(\mathbf{u}, \pi)$ by Theorem 2.5(i) implying that $\mathbf{t}_{\mathrm{nt}}^{+}(\mathbf{u}, \pi)=\mathbf{t}_{\alpha}^{+}(\mathbf{u}, \pi)=\mathbf{t}^{\mathrm{c}+}(\mathbf{u}, \pi) \in L_{p}\left(\partial \Omega, \mathbb{R}^{n}\right)$.

(ii) Let $0<s<1$ first, and the case $s=1$ will follow by inclusion. Under the other hypotheses of item (ii), the canonical conormal derivative, $\mathbf{t}_{\alpha}^{+}(\mathbf{u}, \pi)$, is well defined on the boundary $\partial \Omega$ and belongs to $B_{p, q}^{s-1}\left(\partial \Omega, \mathbb{R}^{n}\right)$. Let $\left\{\Omega_{j}\right\}_{j \geq 1}$ be a sequence 
of sub-domains in $\Omega_{+}$that converge to $\Omega_{+}$in the sense of Lemma 2.2, with the corresponding notations $\Phi_{j}, \nu^{(j)}$ and $\omega_{j}$ also introduced there.

Similar to the proof of Lemma 3.15 in [51], one can now prove that the canonical conormal derivative on $\partial \Omega$ is a limit of the canonical conormal derivatives on $\partial \Omega_{j}$, i.e., $\left\langle\mathbf{t}_{\alpha, \partial \Omega}^{+}(\mathbf{u}, \pi), \gamma_{\partial \Omega+} \mathbf{w}\right\rangle_{\partial \Omega}=\lim _{j \rightarrow \infty}\left\langle\mathbf{t}_{\alpha, \partial \Omega_{j}}^{+}(\mathbf{u}, \pi), \gamma_{\partial \Omega_{j}} \mathbf{w}\right\rangle_{\partial \Omega_{j}}$ for any $\mathbf{w} \in B_{p^{\prime}, q^{\prime}}^{1+\frac{1}{p^{\prime}-s}}\left(\Omega_{+}, \mathbb{R}^{n}\right)$.

The inclusion $(\mathbf{u}, \pi) \in \mathfrak{B}_{p, q, \text { div }}^{s+\frac{1}{p}, t}\left(\Omega_{+}, \mathcal{L}_{\alpha}\right)$ means that the couple $(\mathbf{u}, \pi)$ satisfies the elliptic Brinkman PDE system (2.19) with a right hand side $\mathbf{f} \in B_{p, q}^{t}\left(\Omega_{+}, \mathbb{R}^{n}\right)$, which implies that $(\mathbf{u}, \pi) \in B_{p, q, \text { div }}^{t+2}\left(\Omega_{j}\right) \times B_{p, q}^{t+1}\left(\Omega_{j}\right)$. Then $\gamma_{\partial \Omega_{j}} \boldsymbol{\sigma}(\mathbf{u}, \pi) \in B_{p, q}^{t+1-\frac{1}{p}}\left(\partial \Omega_{j}, \mathbb{R}^{n \times n}\right) \subset$ $L_{p}\left(\partial \Omega_{j}, \mathbb{R}^{n \times n}\right)$ and $\mathbf{t}_{\alpha, \partial \Omega_{j}}^{+}(\mathbf{u}, \pi)=\mathbf{t}_{\partial \Omega_{j}}^{\mathrm{c}}(\mathbf{u}, \pi)=\gamma_{\partial \Omega_{j}}^{+} \boldsymbol{\sigma}(\mathbf{u}, \pi) \boldsymbol{\nu} \in L_{p}\left(\partial \Omega_{j}, \mathbb{R}^{n}\right)$ by item (i).

On the other hand, for a.e. point $x \in \partial \Omega$ the non-tangential function $M(\boldsymbol{\sigma}(\mathbf{u}, \pi))(x)$ exists and is finite, which particularly implies that $\boldsymbol{\sigma}(\mathbf{u}, \pi)$ is well defined and bounded in the approach cones $\mathfrak{D}_{+}(x)$. We can consider $\boldsymbol{\sigma}(\mathbf{u}, \pi)(x)$ as strictly defined (by its limit mean values $\lim _{r \rightarrow 0} f_{B(x, r)} \boldsymbol{\sigma}(\mathbf{u}, \pi)(\xi) d \xi$ in the sense of Jonnson \& Wallin [30, p.15], see also [6, Theorem 8.7]); then $\gamma_{\partial \Omega_{j}} \boldsymbol{\sigma}(\mathbf{u}, \pi)(y)=\boldsymbol{\sigma}(\mathbf{u}, \pi)(y)$ and hence $\mathbf{t}_{\alpha, \partial \Omega_{j}}^{+}(\mathbf{u}, \pi)(y)=\mathbf{t}_{\partial \Omega_{j}}^{\mathrm{c}+}(\mathbf{u}, \pi)(y)=\boldsymbol{\sigma}(\mathbf{u}, \pi)(y) \cdot \boldsymbol{\nu}_{j}(y)$ for $y \in \mathfrak{D}_{+}(x) \cap \partial \Omega_{j}$. In addition $\mathbf{t}_{\alpha, \partial \Omega_{j}}^{+}(\mathbf{u}, \pi)\left(\Phi_{j}(x)\right)=\mathbf{t}_{\partial \Omega_{j}}^{\mathrm{c}+}(\mathbf{u}, \pi)\left(\Phi_{j}(x)\right)=\boldsymbol{\sigma}(\mathbf{u}, \pi)\left(\Phi_{j}(x)\right) \cdot \boldsymbol{\nu}\left(\Phi_{j}(x)\right)$ tends to $\boldsymbol{\sigma}_{\mathrm{nt}}^{+}(\mathbf{u}, \pi)(x) \cdot \boldsymbol{\nu}(x)=\mathbf{t}_{\mathrm{nt}, \partial \Omega}^{+}(\mathbf{u}, \pi)(x)$ as $j \rightarrow \infty$ for a.e. $x \in \partial \Omega$, for which $\boldsymbol{\sigma}_{\mathrm{nt}}^{+}(\mathbf{u}, \pi)(x)$ does exist.

Let us now prove that $\mathbf{t}_{\partial \Omega_{j}}^{\mathrm{c}}(\mathbf{u}, \pi)\left(\Phi_{j}(x)\right)$ converges to $\mathbf{t}_{\mathrm{nt}, \partial \Omega}^{+}(\mathbf{u}, \pi)(x)$ not only point-wise for a.e. $x \in \partial \Omega$ but also in the weak sense, i.e., $\lim _{j \rightarrow \infty}\left\langle\mathbf{t}_{\partial \Omega_{j}}^{\mathrm{c}+}(\mathbf{u}, \pi), \gamma_{\partial} \Omega_{j} \mathbf{w}\right\rangle_{\partial \Omega_{j}}=\left\langle\mathbf{t}_{\mathrm{nt}, \partial \Omega}^{+}(\mathbf{u}, \pi), \gamma_{\partial \Omega+} \mathbf{w}\right\rangle_{\partial \Omega}$ for any $\mathbf{w} \in B_{p^{\prime}, q^{\prime}}^{1+\frac{1}{p^{\prime}}-s}\left(\Omega_{+}, \mathbb{R}^{n}\right)$. We have

$$
\begin{array}{r}
\left|\left\langle\mathbf{t}_{\partial \Omega_{j}}^{\mathrm{c}+}(\mathbf{u}, \pi), \gamma_{\partial \Omega_{j}} \mathbf{w}\right\rangle_{\partial \Omega_{j}}-\left\langle\mathbf{t}_{\mathrm{nt}, \partial \Omega}^{+}(\mathbf{u}, \pi), \gamma_{\partial \Omega+} \mathbf{w}\right\rangle_{\partial \Omega}\right|=\left|\left\langle\mathbf{t}_{\partial \Omega_{j}}^{\mathrm{c}+}(\mathbf{u}, \pi) \circ \Phi_{j}, \omega_{j} \gamma_{\partial \Omega_{j}} \mathbf{w} \circ \Phi_{j}\right\rangle_{\partial \Omega}-\left\langle\mathbf{t}_{\mathrm{nt}, \partial \Omega}^{+}(\mathbf{u}, \pi), \gamma_{\partial \Omega_{+}} \mathbf{w}\right\rangle_{\partial \Omega}\right| \\
\leq\left|\left\langle\mathbf{t}_{\partial \Omega_{j}}^{\mathrm{c}+}(\mathbf{u}, \pi) \circ \Phi_{j}-\mathbf{t}_{\mathrm{nt}, \partial \Omega}^{+}(\mathbf{u}, \pi), \omega_{j} \gamma_{\partial \Omega_{j}} \mathbf{w} \circ \Phi_{j}\right\rangle_{\partial \Omega}\right|+\left|\left\langle\mathbf{t}_{\mathrm{nt}, \partial \Omega}^{+}(\mathbf{u}, \pi),\left(\omega_{j}-1\right) \gamma_{\partial \Omega_{j}} \mathbf{w} \circ \Phi_{j}\right\rangle_{\partial \Omega}\right| \\
+\left|\left\langle\mathbf{t}_{\mathrm{nt}, \partial \Omega}^{+}(\mathbf{u}, \pi), \gamma_{\partial \Omega_{j}} \mathbf{w} \circ \Phi_{j}-\gamma_{\partial \Omega_{+}} \mathbf{w}\right\rangle_{\partial \Omega}\right|
\end{array}
$$

Let us prove that the summands in the right hand side of (2.30) tend to zero as $j \rightarrow \infty$. To this end, we use the inequality

$$
\left|\left\langle\mathbf{t}_{\partial \Omega_{j}}^{\mathrm{c}+}(\mathbf{u}, \pi) \circ \Phi_{j}-\mathbf{t}_{\mathrm{nt}, \partial \Omega}^{+}(\mathbf{u}, \pi), \omega_{j} \gamma_{\partial \Omega_{j}} \mathbf{w} \circ \Phi_{j}\right\rangle_{\partial \Omega}\right| \leq\left\|\mathbf{t}_{\partial \Omega_{j}}^{\mathrm{c}+}(\mathbf{u}, \pi) \circ \Phi_{j}-\mathbf{t}_{\mathrm{nt}, \partial \Omega}^{+}(\mathbf{u}, \pi)\right\|_{L_{p}(\partial \Omega)}\left\|\omega_{j} \gamma_{\partial \Omega_{j}} \mathbf{w} \circ \Phi_{j}\right\|_{L_{p^{\prime}}(\partial \Omega)} .
$$

We have,

$$
\left|\mathbf{t}_{\partial \Omega_{j}}^{c+}(\mathbf{u}, \pi)\left(\Phi_{j}(x)\right)-\mathbf{t}_{\mathrm{nt}, \partial \Omega}^{+}(\mathbf{u}, \pi)(x)\right| \leq M(\boldsymbol{\sigma}(\mathbf{u}, \pi))(x)+\left|\mathbf{t}_{\mathrm{nt}, \partial \Omega}^{+}(\mathbf{u}, \pi)(x)\right|
$$

the both terms in the right hand side of (2.32) belong to $L_{p}(\partial \Omega)$ and $\mathbf{t}_{\partial \Omega_{j}}^{\mathrm{c}}(\mathbf{u}, \pi) \circ \Phi_{j}-\mathbf{t}_{\mathrm{nt}, \partial \Omega}^{+}(\mathbf{u}, \pi) \rightarrow 0$ pointwise a.e. on $\partial \Omega$. Then the Lebesgue dominated convergence theorem implies that the first multiplier in the right hand side of (2.31) tends to zero. Since $\gamma_{\partial \Omega_{j}} \mathbf{w} \in B_{p^{\prime}, q^{\prime}}^{1-s}\left(\partial \Omega_{j}, \mathbb{R}^{n}\right) \subset L_{p^{\prime}}^{1-s}\left(\partial \Omega_{j}, \mathbb{R}^{n}\right)$ and $\gamma_{\partial \Omega_{j}} \mathbf{w} \circ \Phi_{j} \rightarrow \gamma_{\partial \Omega_{+}} \mathbf{w}$ (cf. [64, Chapter 2, Theorem 4.5]), the second multiplier in the right hand side of (2.31) is bounded and hence the whole right hand side of (2.31) tends to zero. The second summand in the right hand side of (2.30) tends to zero since $\omega_{j} \rightarrow 1$, and the third, again, because $\gamma_{\partial \Omega_{j}} \mathbf{w} \circ \Phi_{j} \rightarrow \gamma_{\partial \Omega_{+}} \mathbf{w}$.

Combining this with the previous argument, we obtain,

$$
\left\langle\mathbf{t}_{\alpha, \partial \Omega}^{+}(\mathbf{u}, \pi), \gamma_{\partial \Omega+} \mathbf{w}\right\rangle_{\partial \Omega}=\lim _{j \rightarrow \infty}\left\langle\mathbf{t}_{\partial \Omega_{j}}^{c+}(\mathbf{u}, \pi), \gamma_{\partial \Omega_{j}} \mathbf{w}\right\rangle_{\partial \Omega_{j}}=\left\langle\mathbf{t}_{\mathrm{nt}, \partial \Omega}^{+}(\mathbf{u}, \pi), \gamma_{\partial \Omega+} \mathbf{w}\right\rangle_{\partial \Omega} \quad \forall \mathbf{w} \in B_{p^{\prime}, q^{\prime}}^{1+\frac{1}{p^{\prime}}-s}\left(\Omega_{+}, \mathbb{R}^{n}\right)
$$

Taking $\mathbf{w}=\gamma_{+}^{-1} \boldsymbol{\varphi}$, this gives $\left\langle\mathbf{t}_{\alpha, \partial \Omega}^{+}(\mathbf{u}, \pi), \boldsymbol{\varphi}\right\rangle_{\partial \Omega}=\left\langle\mathbf{t}_{\mathrm{nt}, \partial \Omega}^{+}(\mathbf{u}, \pi), \boldsymbol{\varphi}\right\rangle_{\partial \Omega}$ for any $\boldsymbol{\varphi} \in B_{p^{\prime}, d^{\prime}}^{1-s}\left(\partial \Omega, \mathbb{R}^{n}\right)$, i.e., $\mathbf{t}_{\alpha}^{+}(\mathbf{u}, \pi)=\mathbf{t}_{\mathrm{nt}}^{+}(\mathbf{u}, \pi)$, and since $\mathbf{t}_{\mathrm{nt}}^{+}(\mathbf{u}, \pi)=\boldsymbol{\sigma}_{\mathrm{nt}}^{+}(\mathbf{u}, \pi) \boldsymbol{\nu} \in L_{p}\left(\partial \Omega, \mathbb{R}^{n}\right)$, this completes the proof of item (ii) for $0<s<1$, while for $s=1$ the statement follows by inclusion.

Remark 2.14 Due to Remark 2.12, Theorem 2.13 will still valid for $\Omega_{-}$if the functions belong to the corresponding spaces only locally, i.e., if $(\mathbf{u}, \pi) \in B_{p, q, \text { div,loc }}^{s+\frac{1}{p}}\left(\bar{\Omega}_{-}, \mathbb{R}^{n}\right) \times B_{p, q, \text { loc }}^{s-1+\frac{1}{p}}\left(\bar{\Omega}_{-}\right)$in item (i) and $(\mathbf{u}, \pi) \in \mathfrak{B}_{p, q \text {, div,loc }}^{s+\frac{1}{,}, t}\left(\bar{\Omega}_{-}, \mathcal{L}_{\alpha}\right)$ in item (ii).

\section{Integral potentials for the Brinkman system}

This section is devoted to the main properties of Newtonian and layer potentials for the Brinkman system.

\subsection{Newtonian potential for the Brinkman system}

Let $\alpha>0$ be a constant. Let us denote by $\mathcal{G}^{\alpha}$ and $\Pi$ the fundamental velocity tensor and the fundamental pressure vector for the Brinkman system in $\mathbb{R}^{n}(n \geq 3)$, with the components (see, e.g., [43, (3.6)], [40, Section 3.2.1], [74, (2.14)])

$$
\mathcal{G}_{j k}^{\alpha}(\mathbf{x})=\frac{1}{\tilde{\omega}_{n}}\left\{\frac{\delta_{j k}}{|\mathbf{x}|^{n-2}} A_{1}(\alpha|\mathbf{x}|)+\frac{x_{j} x_{k}}{|\mathbf{x}|^{n}} A_{2}(\alpha|\mathbf{x}|)\right\}, \quad \Pi_{k}(\mathbf{x})=\frac{1}{\tilde{\omega}_{n}} \frac{x_{k}}{|\mathbf{x}|^{n}}
$$


where $A_{1}(z)$ and $A_{2}(z)$ are defined by

$$
A_{1}(z):=\frac{\left(\frac{z}{2}\right)^{\frac{n}{2}-1} K_{\frac{n}{2}-1}(z)}{\Gamma\left(\frac{n}{2}\right)}+2 \frac{\left(\frac{z}{2}\right)^{\frac{n}{2}} K_{\frac{n}{2}}(z)}{\Gamma\left(\frac{n}{2}\right) z^{2}}-\frac{1}{z^{2}}, A_{2}(z):=\frac{n}{z^{2}}-4 \frac{\left(\frac{z}{2}\right)^{\frac{n}{2}+1} K_{\frac{n}{2}+1}(z)}{\Gamma\left(\frac{n}{2}\right) z^{2}},
$$

$K_{\varkappa}$ is the Bessel function of the second kind and order $\varkappa \geq 0, \Gamma$ is the Gamma function, and $\tilde{\omega}_{n}$ is the area of the unit sphere in $\mathbb{R}^{n}$. The fundamental solution of the Stokes system, $(\mathcal{G}, \Pi)$, which corresponds to $\alpha=0$, is given by (see, e.g., [74, (1.12)])

$$
\mathcal{G}_{j k}(\mathbf{x})=\frac{1}{2 \tilde{\omega}_{n}}\left\{\frac{1}{n-2} \frac{\delta_{j k}}{|\mathbf{x}|^{n-2}}+\frac{x_{j} x_{k}}{|\mathbf{x}|^{n}}\right\}, \quad \Pi_{k}(\mathbf{x})=\frac{1}{\tilde{\omega}_{n}} \frac{x_{k}}{|\mathbf{x}|^{n}} .
$$

Next we use the notations $\mathcal{G}^{\alpha}(\mathbf{x}, \mathbf{y})=\mathcal{G}^{\alpha}(\mathbf{x}-\mathbf{y})$ and $\Pi(\mathbf{x}, \mathbf{y})=\Pi(\mathbf{x}-\mathbf{y})$. Then

$$
\left(\triangle_{\mathrm{x}}-\alpha \mathbb{I}\right) \mathcal{G}^{\alpha}(\mathbf{x}, \mathbf{y})-\nabla_{\mathrm{x}} \Pi(\mathbf{x}, \mathbf{y})=-\delta_{\mathrm{y}}(\mathbf{x}) \mathbb{I}, \quad \operatorname{div}_{\mathrm{x}} \mathcal{G}^{\alpha}(\mathbf{x}, \mathbf{y})=0, \quad \forall \mathbf{y} \in \mathbb{R}^{n},
$$

where $\delta_{\mathbf{x}}$ is the Dirac distribution with mass in $\mathbf{y}$, and the subscript $\mathbf{x}$ added to a differential operator refers to the action of that operator with respect to the variable $\mathbf{x}$.

The fundamental stress tensor $\mathbf{S}^{\alpha}$ has the components

$$
S_{i j \ell}^{\alpha}(\mathbf{x}, \mathbf{y})=-\Pi_{j}(\mathbf{x}, \mathbf{y}) \delta_{i \ell}+\frac{\partial \mathcal{G}_{i j}^{\alpha}(\mathbf{x}, \mathbf{y})}{\partial x_{\ell}}+\frac{\partial \mathcal{G}_{\ell j}^{\alpha}(\mathbf{x}, \mathbf{y})}{\partial x_{i}}
$$

where $\delta_{j k}$ is the Kronecker symbol. Let $\Lambda^{\alpha}$ be the fundamental pressure tensor with components $\Lambda_{j k}^{\alpha}$. Then for fixed $i$ and $k$, the pair $\left(S_{i j k}^{\alpha}, \Lambda_{i k}^{\alpha}\right)$ satisfies the Brinkman system in $\mathbb{R}^{n}$ if $\mathbf{x} \neq \mathbf{y}$, i.e.,

$$
\left\{\begin{array}{l}
\triangle_{x} S_{i j k}^{\alpha}(\mathbf{x}, \mathbf{y})-\alpha S_{i j k}^{\alpha}(\mathbf{x}, \mathbf{y})-\frac{\partial \Lambda_{i k}^{\alpha}(\mathbf{y}, \mathbf{x})}{\partial x_{j}}=0, \\
\frac{\partial S_{i j k}^{\alpha}(\mathbf{x}, \mathbf{y})}{\partial x_{j}}=0
\end{array}\right.
$$

The components $\bigwedge_{j k}^{\alpha}(\mathbf{x}, \mathbf{y})$ are given by (see, e.g., $\left.[74,(2.18)]\right)$

$$
\Lambda_{i k}^{\alpha}(\mathbf{x}, \mathbf{y})=\frac{1}{\omega_{n}}\left\{-\left(y_{i}-x_{i}\right) \frac{2 n\left(y_{k}-x_{k}\right)}{|\mathbf{y}-\mathbf{x}|^{n+2}}+\frac{2 \delta_{i k}}{|\mathbf{y}-\mathbf{x}|^{n}}-\alpha \frac{1}{(n-2)} \frac{1}{|\mathbf{y}-\mathbf{x}|^{n-2}} \delta_{i k}\right\} .
$$

For $\alpha=0$, we use the notations $S_{i j k}:=S_{i j k}^{0}$ and $\Lambda_{i k}:=\Lambda_{i k}^{0}$.

Let $*$ denote the convolution product. Let us consider the velocity and pressure Newtonian potential operators for the Brinkman system,

$$
\left(\mathbf{N}_{\alpha ; \mathbb{R}^{n}} \boldsymbol{\varphi}\right)(\mathbf{x}):=-\left(\mathcal{G}^{\alpha} * \boldsymbol{\varphi}\right)(\mathbf{x})=-\left\langle\mathcal{G}^{\alpha}(\mathbf{x}, \cdot), \boldsymbol{\varphi}\right\rangle_{\mathbb{R}^{n}},\left(\mathcal{Q}_{\alpha ; \mathbb{R}^{n}} \boldsymbol{\varphi}\right)(\mathbf{x})=\left(\mathcal{Q}_{\mathbb{R}^{n}} \boldsymbol{\varphi}\right)(\mathbf{x}):=-(\Pi * \boldsymbol{\varphi})(\mathbf{x})=-\langle\Pi(\mathbf{x}, \cdot), \boldsymbol{\varphi}\rangle_{\mathbb{R}^{n}},
$$

where the fundamental tensor $\mathcal{G}^{\alpha}$ is presented through its components in (3.1). Note that the Fourier transform of $\mathcal{G}^{\alpha}$-components is given by

$$
\widehat{\mathcal{G}}_{k j}^{\alpha}(\xi)=\frac{(2 \pi)^{-\frac{n}{2}}}{|\xi|^{2}+\alpha}\left(\delta_{k j}-\frac{\xi_{k} \xi_{j}}{|\xi|^{2}}\right)
$$

Then we have the following property (cf. [43, Theorem 3.10] in the case $n=3, s=0$ ).

Lemma 3.1 Let $\alpha>0$. Then for all $p, q \in(1, \infty)$ and $s \in \mathbb{R}$ the following linear operators are continuous

$$
\begin{aligned}
\mathbf{N}_{\alpha ; \mathbb{R}^{n}} & : H_{p}^{s}\left(\mathbb{R}^{n}, \mathbb{R}^{n}\right) \rightarrow H_{p}^{s+2}\left(\mathbb{R}^{n}, \mathbb{R}^{n}\right), \\
\mathbf{N}_{\alpha ; \mathbb{R}^{n}} & : B_{p, q}^{s}\left(\mathbb{R}^{n}, \mathbb{R}^{n}\right) \rightarrow B_{p, q}^{s+2}\left(\mathbb{R}^{n}, \mathbb{R}^{n}\right), \\
\mathcal{Q}_{\mathbb{R}^{n}} & : H_{p}^{s}\left(\mathbb{R}^{n}, \mathbb{R}^{n}\right) \rightarrow H_{p, \text { loc }}^{s+1}\left(\mathbb{R}^{n}\right), \\
\mathcal{Q}_{\mathbb{R}^{n}} & : B_{p, q}^{s}\left(\mathbb{R}^{n}, \mathbb{R}^{n}\right) \rightarrow B_{p, q, \mathrm{loc}}^{s+1}\left(\mathbb{R}^{n}\right) .
\end{aligned}
$$

Proof. Let $\boldsymbol{\varphi} \in H_{p}^{s}\left(\mathbb{R}^{n}, \mathbb{R}^{n}\right)$. By (2.11),

$$
\left\|\mathbf{N}_{\alpha ; \mathbb{R}^{n}} \boldsymbol{\varphi}\right\|_{H_{p}^{s+2}\left(\mathbb{R}^{n}, \mathbb{R}^{n}\right)}=\left\|\mathcal{F}^{-1}\left(\rho^{s+2} \mathcal{F}\left(\mathbf{N}_{\alpha ; \mathbb{R}^{n}} \boldsymbol{\varphi}\right)\right)\right\|_{L_{p}\left(\mathbb{R}^{n}, \mathbb{R}^{n}\right)},
$$

where $\rho$ is the weight function given by (2.10). In addition, we note that

$$
\mathcal{F}\left(\mathbf{N}_{\alpha ; \mathbb{R}^{n}} \boldsymbol{\varphi}\right)=\mathcal{F}\left(\mathcal{G}^{\alpha} * \boldsymbol{\varphi}\right)=\widehat{\mathcal{G}}^{\alpha} \widehat{\boldsymbol{\varphi}}
$$


and hence by (3.14),

$$
\left\|\mathbf{N}_{\alpha ; \mathbb{R}^{n}} \boldsymbol{\varphi}\right\|_{H_{p}^{s+2}\left(\mathbb{R}^{n}, \mathbb{R}^{n}\right)}=\left\|\mathcal{F}^{-1}\left(\rho^{s+2} \widehat{\mathcal{G}}^{\alpha} \widehat{\boldsymbol{\varphi}}\right)\right\|_{L_{p}\left(\mathbb{R}^{n}, \mathbb{R}^{n}\right)}=\left\|\mathcal{F}^{-1}\left(\widehat{m} \mathcal{F}\left(J^{s} \boldsymbol{\varphi}\right)\right)\right\|_{L_{p}\left(\mathbb{R}^{n}, \mathbb{R}^{n}\right)}
$$

In view of (3.9), the matrix-function $\widehat{m}:=\rho^{2} \widehat{\mathcal{G}}^{\alpha}$ has the components

$$
\widehat{m}_{k j}(\xi)=(2 \pi)^{-\frac{n}{2}} \frac{1+|\xi|^{2}}{|\xi|^{2}+\alpha}\left(\delta_{k j}-\frac{\xi_{k} \xi_{j}}{|\xi|^{2}}\right), k, j=1, \ldots, n,
$$

and is smooth everywhere except the origin and uniformly bounded in $\mathbb{R}^{n} \times \mathbb{R}^{n}$. Hence it is a Fourier multiplier in $L_{p}\left(\mathbb{R}^{n}\right)(\mathrm{cf}$. Theorem 2 in Appendix of [54]), i.e., there exists a constant $M>0$, (which depends on $p$ but is independent of $\boldsymbol{\varphi}$ ) such that

$$
\left\|\mathbf{N}_{\alpha ; \mathbb{R}^{n}} \boldsymbol{\varphi}\right\|_{H_{p}^{s+2}\left(\mathbb{R}^{n}, \mathbb{R}^{n}\right)} \leq M\left\|J^{s} \boldsymbol{\varphi}\right\|_{L_{p}\left(\mathbb{R}^{n}, \mathbb{R}^{n}\right)}=M\|\boldsymbol{\varphi}\|_{H_{p}^{s}\left(\mathbb{R}^{n}, \mathbb{R}^{n}\right)}
$$

and thus $\left\|\mathbf{N}_{\alpha ; \mathbb{R}^{n}}\right\|_{H_{p}^{s}\left(\mathbb{R}^{n}, \mathbb{R}^{n}\right) \rightarrow H_{p}^{s+2}\left(\mathbb{R}^{n}, \mathbb{R}^{n}\right)} \leq M$, while operator (3.10) is continuous.

Moreover, by formula (A.12) we have the interpolation property

$$
\left(H_{p}^{s_{1}}\left(\mathbb{R}^{n}, \mathbb{R}^{n}\right), H_{p}^{s_{2}}\left(\mathbb{R}^{n}, \mathbb{R}^{n}\right)\right)_{\theta, q}=B_{p, q}^{s}\left(\mathbb{R}^{n}, \mathbb{R}^{n}\right), \quad\left(H_{p}^{s_{1}+2}\left(\mathbb{R}^{n}, \mathbb{R}^{n}\right), H_{p}^{s_{2}+2}\left(\mathbb{R}^{n}, \mathbb{R}^{n}\right)\right)_{\theta, q}=B_{p, q}^{s+2}\left(\mathbb{R}^{n}, \mathbb{R}^{n}\right),
$$

where $s=(1-\theta) s_{1}+\theta s_{2}$. Then by continuity of operator (3.10), we obtain that operator (3.11) is also continuous for $p, q \in(1, \infty)$ and any $s \in \mathbb{R}$.

Let us now show the continuity of operators (3.12) and (3.13). To this end, we note that the pressure Newtonian potential operator for the Brinkman system coincides with the one for the Stokes system and for any $\boldsymbol{\varphi} \in \mathcal{D}\left(\mathbb{R}^{n}, \mathbb{R}^{n}\right)$ can be written as

$$
\mathcal{Q}_{\mathbb{R}^{n}} \boldsymbol{\varphi}=\operatorname{div} \mathcal{N}_{\triangle ; \mathbb{R}^{n}} \boldsymbol{\varphi}
$$

where

$$
\left(\mathcal{N}_{\triangle ; \mathbb{R}^{n}} \boldsymbol{\varphi}\right)(\mathbf{x}):=-\left(\mathcal{G}_{\triangle} * \boldsymbol{\varphi}\right)(\mathbf{x})
$$

and $\mathcal{G}_{\triangle}(\mathbf{x}, \mathbf{y}):=-\frac{1}{(n-2) \omega_{n}} \frac{1}{|\mathbf{x}-\mathbf{y}|^{n-2}}$ is the fundamental solution of the Laplace equation in $\mathbb{R}^{n}$. Therefore, the mapping properties of the pressure Newtonian potential are provided by those of the harmonic Newtonian potential $\mathcal{N}_{\triangle ; \mathbb{R}^{n}}$. Since $\mathcal{N}_{\triangle ; \mathbb{R}^{n}}$ is a pseudodifferential operator of order -2 in $\mathbb{R}^{n}$, the following operator is continuous,

$$
\mathcal{N}_{\triangle ; \mathbb{R}^{n}}: H_{p}^{s}\left(\mathbb{R}^{n}\right) \rightarrow H_{p, \text { loc }}^{s+2}\left(\mathbb{R}^{n}\right), \forall s \in \mathbb{R}, p \in(1, \infty) .
$$

Then by (3.18) and (3.20) we deduce the continuity property of the pressure Newtonian potential operator in (3.12). By using an interpolation argument as for (3.11), we also obtain continuity of operator (3.13).

Let $\alpha \geq 0$ and $p \in(1, \infty)$ be given. The Newtonian velocity and pressure potential operators of the Brinkman system in Lipschitz domains $\Omega_{ \pm}$are defined as

$$
\mathbf{N}_{\alpha ; \Omega}=r_{\Omega} \mathbf{N}_{\alpha ; \mathbb{R}^{n}} \stackrel{\circ}{E_{ \pm}} \quad \text { and } \quad \mathcal{Q}_{\Omega_{ \pm}}=r_{\Omega_{ \pm}} \mathcal{Q}_{\mathbb{R}^{n}} \stackrel{\circ}{E}_{ \pm}
$$

Recall that $\dot{E}_{ \pm}$is the operator of extension of vector fields defined in $\Omega_{ \pm}$by zero on $\mathbb{R}^{n} \backslash \Omega_{ \pm}$, and $r_{\Omega_{ \pm}}$is the restriction operator from $\mathbb{R}^{n}$ to $\Omega_{ \pm}$. The operators $\dot{E}_{ \pm}: L_{p}\left(\Omega_{ \pm}, \mathbb{R}^{n}\right) \rightarrow L_{p}\left(\mathbb{R}^{n}, \mathbb{R}^{n}\right)$ and $r_{\Omega_{ \pm}}: H_{p}^{2}\left(\mathbb{R}^{n}, \mathbb{R}^{n}\right) \rightarrow H_{p}^{2}\left(\Omega_{ \pm}, \mathbb{R}^{n}\right)$ are linear and continuous. In addition, the volume potential operator $\mathbf{N}_{\alpha ; \mathbb{R}^{n}}: L_{p}\left(\mathbb{R}^{n}, \mathbb{R}^{n}\right) \rightarrow H_{p}^{2}\left(\mathbb{R}^{n}, \mathbb{R}^{n}\right)$ is linear and continuous as well, for any $p \in(1, \infty)$ (cf., e.g., [43, Theorem 3.10], [20, Lemma 1.3] and Lemma 3.1). Therefore, the velocity Newtonian potential operators

$$
\mathbf{N}_{\alpha ; \Omega_{ \pm}}: L_{p}\left(\Omega_{ \pm}, \mathbb{R}^{n}\right) \rightarrow H_{p}^{2}\left(\Omega_{ \pm}, \mathbb{R}^{n}\right), \quad p \in(1, \infty)
$$

are continuous operators. A similar argument yields the continuity of the Newtonian pressure potential operators

$$
\mathcal{Q}_{\Omega_{+}}: L_{p}\left(\Omega_{+}, \mathbb{R}^{n}\right) \rightarrow H_{p}^{1}\left(\Omega_{+}\right), \quad \mathcal{Q}_{\Omega_{-}}: L_{p}\left(\Omega_{-}, \mathbb{R}^{n}\right) \rightarrow H_{p, \text { loc }}^{1}\left(\Omega_{-}\right), \quad p \in(1, \infty) .
$$

Next, in view of (A.5), (A.6) and the first inclusion in (A.8) we obtain the inclusions

$$
H_{p}^{2}\left(\mathbb{R}^{n}, \mathbb{R}^{n}\right)=W_{p}^{2}\left(\mathbb{R}^{n}, \mathbb{R}^{n}\right) \hookrightarrow W_{p}^{1+\frac{1}{p}}\left(\mathbb{R}^{n}, \mathbb{R}^{n}\right)=B_{p, p}^{1+\frac{1}{p}}\left(\mathbb{R}^{n}, \mathbb{R}^{n}\right) \hookrightarrow B_{p, p^{*}}^{1+\frac{1}{p}}\left(\mathbb{R}^{n}, \mathbb{R}^{n}\right), \quad \forall p \geq 1, p^{*}=\max \{p, 2\},
$$

which are continuous. Then relations (3.22) and (3.24) imply also the continuity of the velocity Newtonian potential operator

$$
\mathbf{N}_{\alpha ; \Omega_{ \pm}}: L_{p}\left(\Omega_{ \pm}, \mathbb{R}^{n}\right) \rightarrow B_{p, p^{*}}^{1+\frac{1}{p}}\left(\Omega_{ \pm}, \mathbb{R}^{n}\right), \quad p \in(1, \infty) .
$$

A similar argument yields the continuity property of the pressure Newtonian potential operator

$$
\mathcal{Q}_{\alpha ; \Omega_{+}}: L_{p}\left(\Omega_{+}, \mathbb{R}^{n}\right) \rightarrow B_{p, p^{*}}^{\frac{1}{p}}\left(\Omega_{+}\right), \quad \mathcal{Q}_{\alpha ; \Omega_{-}}: L_{p}\left(\Omega_{-}, \mathbb{R}^{n}\right) \rightarrow B_{p, p^{*}, \text { loc }}^{\frac{1}{\bar{p}}}\left(\bar{\Omega}_{-}\right), \quad p \in(1, \infty) .
$$

In addition, due to (3.21), we have the relations

$$
\triangle \mathbf{N}_{\alpha ; \Omega_{ \pm}} \mathbf{f}-\alpha \mathbf{N}_{\alpha ; \Omega_{ \pm}} \mathbf{f}-\nabla \mathcal{Q}_{\Omega_{ \pm}} \mathbf{f}=\mathbf{f}, \operatorname{div} \mathbf{N}_{\alpha ; \Omega_{ \pm}} \mathbf{f}=0 \text { in } \Omega_{ \pm} .
$$

This leads us to the following assertion. 
Corollary 3.2 Let $\alpha>0, p \in(1, \infty)$, and $p^{*}=\max \{p, 2\}$. Then the Brinkman Newtonian potentials satisfy equations (6.46) and the following operators are continuous

$$
\begin{aligned}
\left(\mathbf{N}_{\alpha ; \Omega_{+}}, \mathcal{Q}_{\Omega_{+}}\right): L_{p}\left(\Omega_{+}, \mathbb{R}^{n}\right) \rightarrow \mathfrak{H}_{p, \text { div }}^{2,0}\left(\Omega_{+}, \mathcal{L}_{\alpha}\right), & \left(\mathbf{N}_{\alpha ; \Omega_{-}}, \mathcal{Q}_{\Omega_{-}}\right): L_{p}\left(\Omega_{-}, \mathbb{R}^{n}\right) \rightarrow \mathfrak{H}_{p, \text { div,loc }}^{2,0}\left(\bar{\Omega}_{-}, \mathcal{L}_{\alpha}\right), \\
\left(\mathbf{N}_{\alpha ; \Omega_{+}}, \mathcal{Q}_{\Omega_{+}}\right): L_{p}\left(\Omega_{+}, \mathbb{R}^{n}\right) \rightarrow \mathfrak{B}_{p, p^{*}, \operatorname{div}}^{2,0}\left(\Omega_{+}, \mathcal{L}_{\alpha}\right), & \left(\mathbf{N}_{\alpha ; \Omega_{-}}, \mathcal{Q}_{\Omega_{-}}\right): L_{p}\left(\Omega_{-}, \mathbb{R}^{n}\right) \rightarrow \mathfrak{B}_{p, p^{*}, \operatorname{div}, \text { loc }}^{2}\left(\bar{\Omega}_{-}, \mathcal{L}_{\alpha}\right) .
\end{aligned}
$$

Remark 3.3 Let $\mathbf{f}_{ \pm} \in L_{p}\left(\Omega_{ \pm}, \mathbb{R}^{n}\right)$ for some $p \in(1, \infty)$, and $p^{*}=\max \{p, 2\}$. Then Corollary 3.2, Lemmas $2.4,2.11$ and Remark 2.12 imply that

$$
\gamma_{ \pm}\left(\mathbf{N}_{\alpha ; \Omega_{ \pm}} \mathbf{f}_{ \pm}\right) \in B_{p, p^{*} ; \nu}^{s}\left(\partial \Omega, \mathbb{R}^{n}\right), \quad \mathbf{t}_{\alpha}^{ \pm}\left(\mathbf{N}_{\alpha ; \Omega_{ \pm}} \mathbf{f}_{ \pm}, \mathcal{Q}_{\Omega_{ \pm}} \mathbf{f}_{ \pm}\right) \in B_{p, p^{*}}^{s-1}\left(\partial \Omega, \mathbb{R}^{n}\right), \quad \forall s \in(0,1) .
$$

Moreover, due to (3.22), the first equality in (3.24), Theorem 2.13, and [10, Theorem 5], these inclusions can be improved to the following ones

$$
\gamma_{ \pm}\left(\mathbf{N}_{\alpha ; \Omega_{ \pm}} \mathbf{f}_{ \pm}\right) \in H_{p ; \nu}^{1}\left(\partial \Omega, \mathbb{R}^{n}\right), \mathbf{t}_{\alpha}^{ \pm}\left(\mathbf{N}_{\alpha ; \Omega_{ \pm}} \mathbf{f}_{ \pm}, \mathcal{Q}_{\Omega_{ \pm}} \mathbf{f}_{ \pm}\right)=\mathbf{t}^{c \pm}\left(\mathbf{N}_{\alpha ; \Omega_{ \pm}} \mathbf{f}_{ \pm}, \mathcal{Q}_{\Omega_{ \pm}} \mathbf{f}_{ \pm}\right) \in L_{p}\left(\partial \Omega, \mathbb{R}^{n}\right)
$$

In (3.30), (3.31) and further on, the following space notations are used for $p \in(1, \infty), q \in(1, \infty], s \in(0,1]$, and the outward unit normal $\boldsymbol{\nu}$ to the Lipschitz domain $\Omega_{+} \subset \mathbb{R}^{n}$,

$$
\begin{aligned}
& L_{p ; \nu}\left(\partial \Omega, \mathbb{R}^{n}\right):=\left\{\mathbf{v} \in L_{p}\left(\partial \Omega, \mathbb{R}^{n}\right): \int_{\partial \Omega} \mathbf{v} \cdot \boldsymbol{\nu} d \sigma=0\right\}, H_{p ; \boldsymbol{\nu}}^{s}\left(\partial \Omega, \mathbb{R}^{n}\right):=\left\{\mathbf{v} \in H_{p}^{s}\left(\partial \Omega, \mathbb{R}^{n}\right): \int_{\partial \Omega} \mathbf{v} \cdot \boldsymbol{\nu} d \sigma=0\right\}, \\
& B_{p, q ; \nu}^{s}\left(\partial \Omega, \mathbb{R}^{n}\right):=\left\{\mathbf{v} \in B_{p, q}^{s}\left(\partial \Omega, \mathbb{R}^{n}\right): \int_{\partial \Omega} \mathbf{v} \cdot \boldsymbol{\nu} d \sigma=0\right\} .
\end{aligned}
$$

\subsection{Layer potentials for the Brinkman system}

For a given density $\mathbf{g} \in L_{p}\left(\partial \Omega, \mathbb{R}^{n}\right)$, the velocity single-layer potential for the Brinkman system, $\mathbf{V}_{\alpha} \mathbf{g}$, and the corresponding pressure single-layer potential, $Q^{s} \mathbf{g}$, are given by

$$
\left(\mathbf{V}_{\alpha} \mathbf{g}\right)(\mathbf{x}):=\left\langle\mathcal{G}^{\alpha}(\mathbf{x}, \cdot), \mathbf{g}\right\rangle_{\partial \Omega},\left(\mathcal{Q}^{s} \mathbf{g}\right)(\mathbf{x}):=\langle\Pi(\mathbf{x}, \cdot), \mathbf{g}\rangle_{\partial \Omega}, \mathbf{x} \in \mathbb{R}^{n} \backslash \partial \Omega
$$

Let $\mathbf{h} \in H_{p}^{1}\left(\partial \Omega, \mathbb{R}^{n}\right)$ be a given density. Then the velocity double-layer potential, $\mathbf{W}_{\alpha ; \partial \Omega} \mathbf{h}$, and the corresponding pressure double-layer potential, $Q_{\alpha ; \partial \Omega}^{d} \mathbf{h}$, are defined by

$$
\left(\mathbf{W}_{\alpha} \mathbf{h}\right)_{j}(\mathbf{x}):=\int_{\partial \Omega} S_{i j \ell}^{\alpha}(\mathbf{x}, \mathbf{y}) \nu_{\ell}(\mathbf{y}) h_{i}(\mathbf{y}) d \sigma_{y},\left(\mathcal{Q}_{\alpha}^{d} \mathbf{h}\right)(\mathbf{x}):=\int_{\partial \Omega} \Lambda_{j \ell}^{\alpha}(\mathbf{x}, \mathbf{y}) \nu_{\ell}(\mathbf{y}) h_{j}(\mathbf{y}) d \sigma_{y}, \quad \forall \mathbf{x} \in \mathbb{R}^{n} \backslash \partial \Omega,
$$

where $\nu_{\ell}, \ell=1, \ldots, n$, are the components of the outward unit normal $\boldsymbol{\nu}$ to $\Omega_{+}$, which is defined a.e. (with respect to the surface measure $\sigma$ ) on $\partial \Omega$. Note that the definition of the double layer potential in [69, (3.9)] differs from definition (3.34) due to different conormal derivatives used in $[69,(1.14)]$ and in formula (2.22) of our paper.

The single- and double-layer potentials can be also defined for any $\mathbf{g} \in B_{p, q}^{s-1}\left(\partial \Omega, \mathbb{R}^{n}\right)$ and $\mathbf{h} \in B_{p, q}^{s}\left(\partial \Omega, \mathbb{R}^{n}\right)$, respectively, where $s \in(0,1)$ and $p, q \in(1, \infty)$. For $\alpha=0$ (i.e., for the Stokes system) we use the notations $\mathbf{V g}, \mathcal{Q}^{s} \mathbf{g}, \mathbf{W h}$ and $\mathcal{Q}^{d} \mathbf{h}$ for the corresponding single- and double-layer potentials.

In view of equations (3.4) and (3.6), the pairs $\left(\mathbf{V}_{\alpha} \mathbf{g}, Q^{s} \mathbf{g}\right)$ and $\left(\mathbf{W}_{\alpha}^{s} \mathbf{h}, Q_{\alpha}^{d} \mathbf{h}\right)$ satisfy the homogeneous Brinkman system in $\Omega_{ \pm}$,

$$
\begin{aligned}
& (\triangle-\alpha \mathbb{I}) \mathbf{V}_{\alpha} \mathbf{g}-\nabla \mathcal{Q}^{s} \mathbf{g}=\mathbf{0}, \quad \operatorname{div} \mathbf{V}_{\alpha} \mathbf{g}=0 \quad \text { in } \mathbb{R}^{n} \backslash \partial \Omega, \\
& (\triangle-\alpha \mathbb{I}) \mathbf{W}_{\alpha} \mathbf{h}-\nabla \mathcal{Q}^{s} \mathbf{h}=\mathbf{0}, \quad \operatorname{div} \mathbf{W}_{\alpha} \mathbf{h}=0 \quad \text { in } \mathbb{R}^{n} \backslash \partial \Omega
\end{aligned}
$$

The direct value of the double layer potential $\mathbf{W}_{\alpha ; \partial \Omega} \mathbf{h}$ on the boundary is defined in terms of Cauchy principal value by

$$
\left(\mathbf{K}_{\alpha} \mathbf{h}\right)_{k}(\mathbf{x}):=\text { p.v. } \int_{\partial \Omega} S_{j k \ell}^{\alpha}(\mathbf{y}, \mathbf{x}) \nu_{\ell}(\mathbf{y}) h_{j}(\mathbf{y}) d \sigma_{\mathrm{y}} \text { a.e. } \mathbf{x} \in \partial \Omega .
$$

Lemma 3.4 Let $\Omega_{+} \subset \mathbb{R}^{n}(n \geq 3)$ be a bounded Lipschitz domain with connected boundary $\partial \Omega$ and let $\Omega_{-}:=\mathbb{R}^{n} \backslash \bar{\Omega}_{+}$. Let $\alpha \geq 0$ and $p \in(1, \infty)$. There exist some constants $C_{i}>0, i=1, \ldots, 4$, depending only on $p, \alpha$ and the Lipschitz character of $\Omega_{+}$, such that the following properties hold:

$$
\begin{aligned}
& \left\|M\left(\nabla \mathbf{V}_{\alpha} \mathbf{g}\right)\right\|_{L_{p}(\partial \Omega)}+\left\|M\left(\mathbf{V}_{\alpha} \mathbf{g}\right)\right\|_{L_{p}(\partial \Omega)}+\left\|M\left(\mathcal{Q}^{s} \mathbf{g}\right)\right\|_{L_{p}(\partial \Omega)} \leq C_{1}\|\mathbf{g}\|_{L_{p}\left(\partial \Omega, \mathbb{R}^{n}\right)}, \quad \forall \mathbf{g} \in L_{p}\left(\partial \Omega, \mathbb{R}^{n}\right), \\
& \left\|M\left(\mathbf{V}_{\alpha} \mathbf{g}\right)\right\|_{L_{p}(\partial \Omega)} \leq C_{2}\|\mathbf{g}\|_{H_{p}^{-1}\left(\partial \Omega, \mathbb{R}^{n}\right)}, \quad \forall \mathbf{g} \in H_{p}^{-1}\left(\partial \Omega, \mathbb{R}^{n}\right), \\
& \left\|M\left(\mathbf{W}_{\alpha} \mathbf{h}\right)\right\|_{L_{p}(\partial \Omega)} \leq C_{3}\|\mathbf{h}\|_{L_{p}\left(\partial \Omega, \mathbb{R}^{n}\right)}, \quad \forall \mathbf{h} \in L_{p}\left(\partial \Omega, \mathbb{R}^{n}\right), \\
& \left\|M\left(\nabla \mathbf{W}_{\alpha} \mathbf{h}\right)\right\|_{L_{p}(\partial \Omega)}+\left\|M\left(\mathbf{W}_{\alpha} \mathbf{h}\right)\right\|_{L_{p}(\partial \Omega)}+\left\|M\left(\mathcal{Q}_{\alpha}^{d} \mathbf{h}\right)\right\|_{L_{p}(\partial \Omega)} \leq C_{4}\|\mathbf{h}\|_{H_{p}^{1}\left(\partial \Omega, \mathbb{R}^{n}\right)}, \quad \forall \mathbf{h} \in H_{p}^{1}\left(\partial \Omega, \mathbb{R}^{n}\right) .
\end{aligned}
$$


Moreover, the following estimates hold for the non-tangential traces that exist at almost all points of $\partial \Omega$ :

$$
\begin{aligned}
& \left\|\left(\mathbf{V}_{\alpha} \mathbf{g}\right)_{\mathrm{nt}}^{ \pm}\right\|_{L_{p}\left(\partial \Omega, \mathbb{R}^{n}\right)},\left\|\left(\nabla \mathbf{V}_{\alpha} \mathbf{g}\right)_{\mathrm{nt}}^{ \pm}\right\|_{L_{p}\left(\partial \Omega, \mathbb{R}^{n}\right)},\left\|\left(\mathcal{Q}^{s} \mathbf{g}\right)_{\mathrm{nt}}^{ \pm}\right\|_{L_{p}\left(\partial \Omega, \mathbb{R}^{n}\right)} \leq C_{1}\|\mathbf{g}\|_{L_{p}\left(\partial \Omega, \mathbb{R}^{n}\right)}, \quad \forall \mathbf{g} \in L_{p}\left(\partial \Omega, \mathbb{R}^{n}\right), \\
& \left\|\left(\mathbf{V}_{\alpha} \mathbf{g}\right)_{\mathrm{nt}}^{ \pm}\right\|_{L_{p}(\partial \Omega)} \leq C_{2}\|\mathbf{g}\|_{H_{p}^{-1}\left(\partial \Omega, \mathbb{R}^{n}\right)}, \quad \forall \mathbf{g} \in H_{p}^{-1}\left(\partial \Omega, \mathbb{R}^{n}\right), \\
& \left\|\left(\mathbf{W}_{\alpha} \mathbf{g}\right)_{\mathrm{nt}}^{ \pm}\right\|_{L_{p}\left(\partial \Omega, \mathbb{R}^{n}\right)} \leq C_{3}\|\mathbf{h}\|_{L_{p}\left(\partial \Omega, \mathbb{R}^{n}\right)}, \quad \forall \mathbf{h} \in L_{p}\left(\partial \Omega, \mathbb{R}^{n}\right), \\
& \left\|\left(\mathbf{W}_{\alpha} \mathbf{h}\right)_{\mathrm{nt}}^{ \pm}\right\|_{L_{p}\left(\partial \Omega, \mathbb{R}^{n}\right)},\left\|\left(\nabla \mathbf{W}_{\alpha} \mathbf{h}\right)_{\mathrm{nt}}^{ \pm}\right\|_{L_{p}\left(\partial \Omega, \mathbb{R}^{n}\right)},\left\|\left(\mathcal{Q}_{\alpha}^{d} \mathbf{h}\right)_{\mathrm{nt}}^{ \pm}\right\|_{L_{p}\left(\partial \Omega, \mathbb{R}^{n}\right)} \leq C_{4}\|\mathbf{h}\|_{H_{p}^{1}\left(\partial \Omega, \mathbb{R}^{n}\right)}, \quad \forall \mathbf{h} \in H_{p}^{1}\left(\partial \Omega, \mathbb{R}^{n}\right) .
\end{aligned}
$$

Proof. In the case $\alpha=0$, inequalities (3.38)-(3.41) follow from [61, Propositions 4.2.3 and 4.2.8].

In the case $\alpha>0$, Inequality (3.38) has been obtained in [69, Lemma 3.2]. In addition, inequality (3.39) follows by the same arguments as in the proof of its counterpart in the case $\alpha=0$ (cf. [61, (4.61)]). Indeed, if $\mathbf{g} \in H_{p}^{-1}\left(\partial \Omega, \mathbb{R}^{n}\right)$, then there exist $\mathbf{g}_{0}=\left(g_{0 ; 1}, \ldots, g_{0 ; n}\right), \mathbf{g}_{r \ell}=\left(g_{r \ell ; 1}, \ldots, g_{r \ell ; n}\right) \in L_{p}\left(\partial \Omega, \mathbb{R}^{n}\right), r, \ell=1, \ldots, n$, such that

$$
g_{k}=g_{0 ; k}+\sum_{r, \ell=1}^{n} \partial_{\tau_{r \ell}} g_{r \ell ; k},\left\|g_{0 ; k}\right\|_{L_{p}(\partial \Omega)}+\sum_{r, \ell=1}^{n}\left\|g_{r \ell ; k}\right\|_{L_{p}(\partial \Omega)} \leq 2\left\|g_{k}\right\|_{H_{p}^{-1}(\partial \Omega)}, \quad k=1, \ldots, n,
$$

(cf. [61, Corollary 2.1.2 and relation (4.65)]), where $\partial_{\tau_{r \ell}}=\nu_{r} \partial_{\ell}-\nu_{\ell} \partial_{r}$ are the tangential derivative operators. Hence, integrating by parts,

$$
\left(\mathbf{V}_{\alpha} \mathbf{g}\right)_{j}(\mathbf{x})=\int_{\partial \Omega} \mathcal{G}_{j k}^{\alpha}(\mathbf{x}-\mathbf{y}) g_{0 ; k}(\mathbf{y}) d \sigma_{y}-\sum_{k=1}^{n} \sum_{r, \ell=1}^{n} \int_{\partial \Omega}\left(\partial_{\tau_{r \ell}}\left(\mathcal{G}_{j k}^{\alpha}(\mathbf{x}-\mathbf{y})\right)\right) g_{r \ell ; k}(\mathbf{y}) d \sigma_{y}, \forall \mathbf{x} \in \mathbb{R}^{n} \backslash \partial \Omega
$$

(cf. [61, (4.66)] for $\alpha=0$ ). Inequality (3.39) immediately follows from equality (3.47) and the estimates in (3.38) and (3.46).

Let us now show inequality (3.40) for $\alpha>0$ (note that its analogue for a differently defined double layer potential in place of $\mathbf{W}_{\alpha}$ was given in [69, Theorem 3.5]). First, we note that Lemma 4.1 in [46] (see also [69, Theorem 2.5]) implies that there exists a constant $c_{\alpha}=c_{\alpha}\left(\Omega_{+}, \alpha\right)>0$ such that

$$
\left|\nabla \mathcal{G}^{\alpha}(\mathbf{x}, \mathbf{y})-\nabla \mathcal{G}(\mathbf{x}, \mathbf{y})\right| \leq c_{\alpha}|\mathbf{x}-\mathbf{y}|^{2-n}, \quad \forall \mathbf{x}, \mathbf{y} \in \bar{\Omega}_{+}, \mathbf{x} \neq \mathbf{y} .
$$

Then, in view of formula (3.5) and equality $\Pi^{\alpha}=\Pi$, there exists a constant $C_{5}=C_{5}\left(\Omega_{+}, \alpha\right)>0$ such that

$$
\left|S_{i j k}^{\alpha}(\mathbf{y}, \mathbf{x})-S_{i j k}(\mathbf{y}, \mathbf{x})\right| \leq\left|\frac{\partial \mathcal{G}_{i j}^{\alpha}(\mathbf{y}, \mathbf{x})}{\partial y_{k}}-\frac{\partial \mathcal{G}_{i j}(\mathbf{y}, \mathbf{x})}{\partial y_{k}}\right|+\left|\frac{\partial \mathcal{G}_{k j}^{\alpha}(\mathbf{y}, \mathbf{x})}{\partial y_{i}}-\frac{\partial \mathcal{G}(\mathbf{y}, \mathbf{x})}{\partial y_{i}}\right| \leq C_{5}|\mathbf{x}-\mathbf{y}|^{2-n}, \quad \forall \mathbf{x}, \mathbf{y} \in \bar{\Omega}_{+}, \mathbf{x} \neq \mathbf{y} .
$$

Inequality (3.49) and [47, Proposition 1] (applied to the integral operator $\mathbf{W}_{\alpha}-\mathbf{W}$ whose kernel is $\left(\mathbf{S}^{\alpha}(\mathbf{y}, \mathbf{x})-\mathbf{S}(\mathbf{y}, \mathbf{x})\right) \boldsymbol{\nu}(\mathbf{y})$ ) show that there exists a constant $C_{6}=C_{6}(\partial \Omega, p, \alpha)>0$ such that

$$
\left\|M\left(\left(\mathbf{W}_{\alpha}-\mathbf{W}\right) \mathbf{h}\right)\right\|_{L_{p}(\partial \Omega)} \leq C_{6}\|\mathbf{h}\|_{L_{p}\left(\partial \Omega, \mathbb{R}^{n}\right)}, \forall \mathbf{h} \in L_{p}\left(\partial \Omega, \mathbb{R}^{n}\right)
$$

Moreover, by [61, (4.56)], there exists a constant $C_{7}=C_{7}(\partial \Omega, p)>0$ such that

$$
\|M(\mathbf{W h})\|_{L_{p}(\partial \Omega)} \leq C_{7}\|\mathbf{h}\|_{L_{p}\left(\partial \Omega, \mathbb{R}^{n}\right)}, \forall \mathbf{h} \in L_{p}\left(\partial \Omega, \mathbb{R}^{n}\right),
$$

and then, by (3.50) and (3.51), we obtain inequality (3.40).

Let us now show inequality (3.41) for $\alpha>0$. According to the second formula in (3.34) and formula (3.7) the kernel of the Brinkman double-layer pressure potential operator $\mathcal{Q}_{\alpha}^{d}$ is given by

$$
\Lambda_{j k}^{\alpha}(\mathbf{x}, \mathbf{y}) \nu_{k}(\mathbf{y})=\frac{1}{\tilde{\omega}_{n}}\left\{-\frac{2 n\left(y_{j}-x_{j}\right)\left(y_{k}-x_{k}\right) \nu_{k}(\mathbf{y})}{|\mathbf{y}-\mathbf{x}|^{n+2}}+\frac{2 \nu_{j}(\mathbf{y})}{|\mathbf{y}-\mathbf{x}|^{n}}-\alpha \frac{1}{(n-2)} \frac{1}{|\mathbf{y}-\mathbf{x}|^{n-2}} \nu_{j}(\mathbf{y})\right\} .
$$

For $\alpha=0$, (3.52) reduces to the kernel of the Stokes double-layer pressure potential operator $\mathcal{Q}^{d}$. Therefore,

$$
\left|\Lambda_{j k}^{\alpha}(\mathbf{x}, \mathbf{y}) \nu_{k}(\mathbf{y})-\Lambda_{j k}(\mathbf{x}, \mathbf{y}) \nu_{k}(\mathbf{y})\right| \leq \frac{\alpha}{\tilde{\omega}_{n}(n-2)} \frac{1}{|\mathbf{y}-\mathbf{x}|^{n-2}}, \quad \forall \mathbf{x} \in \bar{\Omega}_{+}, \mathbf{y} \in \partial \Omega, \mathbf{x} \neq \mathbf{y} .
$$

Then according to [47, Proposition 1] applied to the operator $\mathcal{Q}_{\alpha}^{d}-\mathcal{Q}^{d}$, there exists a constant $C_{8}=C_{8}(\partial \Omega, p, \alpha)$ such that

$$
\left\|M\left(\left(\mathcal{Q}_{\alpha}^{d}-\mathcal{Q}^{d}\right) \mathbf{h}\right)\right\|_{L_{p}(\partial \Omega)} \leq C_{8}\|\mathbf{h}\|_{L_{p}\left(\partial \Omega, \mathbb{R}^{n}\right)}, \forall \mathbf{h} \in H_{p}^{1}\left(\partial \Omega, \mathbb{R}^{n}\right) .
$$

In view of [61, Proposition 4.2.8], the Stokes double-layer pressure potential operator $\mathcal{Q}^{d}$ satisfies the inequality

$$
\left\|M\left(\mathcal{Q}^{d} \mathbf{h}\right)\right\|_{L_{p}(\partial \Omega)} \leq C_{9}\|\mathbf{h}\|_{H_{p}^{1}\left(\partial \Omega, \mathbb{R}^{n}\right)}, \forall \mathbf{h} \in H_{p}^{1}\left(\partial \Omega, \mathbb{R}^{n}\right)
$$


with a constant $C_{9} \equiv C_{9}(\partial \Omega, p)>0$. Then by (3.54) and (3.55) there exists a constant $C_{10} \equiv C_{10}(\partial \Omega, p, \alpha)>0$ such that

$$
\left\|M\left(\mathcal{Q}_{\alpha}^{d} \mathbf{h}\right)\right\|_{L_{p}(\partial \Omega)} \leq C_{10}\|\mathbf{h}\|_{H_{p}^{1}\left(\partial \Omega, \mathbb{R}^{n}\right)}, \forall \mathbf{h} \in H_{p}^{1}\left(\partial \Omega, \mathbb{R}^{n}\right) .
$$

Next, we show that there exists a constant $c_{3}=c_{3}\left(\Omega_{+}, p, \alpha\right)>0$ such that

$$
\left\|M\left(\nabla \mathbf{W}_{\alpha} \mathbf{h}\right)\right\|_{L_{p}(\partial \Omega)} \leq c_{3}\|\mathbf{h}\|_{H_{p}^{1}\left(\partial \Omega, \mathbb{R}^{n}\right)}, \forall \mathbf{h} \in H_{p}^{1}\left(\partial \Omega, \mathbb{R}^{n}\right)
$$

To this end, we use expressions (3.34) and (3.5) for the Brinkman double layer potential $\mathbf{W}_{\alpha} \mathbf{h}$ to obtain for any $\mathbf{h} \in H_{p}^{1}\left(\partial \Omega, \mathbb{R}^{n}\right)$,

$$
\begin{aligned}
\partial_{r}\left(\mathbf{W}_{\alpha} \mathbf{h}\right)_{j}(\mathbf{x})= & -\int_{\partial \Omega}\left\{\nu_{\ell}(\mathbf{y})\left(\partial_{r} \partial_{\ell} \mathcal{G}_{j k}^{\alpha}\right)(\mathbf{y}-\mathbf{x})+\nu_{\ell}(\mathbf{y})\left(\partial_{r} \partial_{j} \mathcal{G}_{\ell k}^{\alpha}\right)(\mathbf{y}-\mathbf{x})-\nu_{j}(\mathbf{y})\left(\partial_{r} \Pi_{k}\right)(\mathbf{y}-\mathbf{x})\right\} h_{k}(\mathbf{y}) d \sigma_{y} \\
= & -\int_{\partial \Omega}\left\{\partial_{\tau_{\ell r}(y)}\left(\partial_{\ell} \mathcal{G}_{j k}^{\alpha}\right)(\mathbf{y}-\mathbf{x})+\partial_{\tau_{\ell r}(y)}\left(\partial_{j} \mathcal{G}_{\ell k}^{\alpha}\right)(\mathbf{y}-\mathbf{x})-\partial_{\tau_{j r}(y)} \Pi_{k}(\mathbf{y}-\mathbf{x})\right\} h_{k}(\mathbf{y}) d \sigma_{y} \\
& -\int_{\partial \Omega}\left\{\nu_{r}(\mathbf{y}) \triangle \mathcal{G}_{j k}^{\alpha}(\mathbf{y}-\mathbf{x})+\nu_{r}(\mathbf{y})\left(\partial_{\ell} \partial_{j} \mathcal{G}_{\ell k}^{\alpha}\right)(\mathbf{y}-\mathbf{x})-\nu_{r}(\mathbf{y})\left(\partial_{j} \Pi_{k}\right)(\mathbf{y}-\mathbf{x})\right\} h_{k}(\mathbf{y}) d \sigma_{y} \\
= & \int_{\partial \Omega}\left\{\left(\partial_{\ell} \mathcal{G}_{j k}^{\alpha}\right)(\mathbf{y}-\mathbf{x})\left(\partial_{\tau_{\ell r}} h_{k}\right)(\mathbf{y})+\left(\partial_{j} \mathcal{G}_{\ell k}^{\alpha}\right)(\mathbf{y}-\mathbf{x})\left(\partial_{\tau_{\ell r}} h_{k}\right)(\mathbf{y})-\Pi_{k}(\mathbf{y}-\mathbf{x})\left(\partial_{\tau_{j r}} h_{k}\right)(\mathbf{y})\right\} d \sigma_{y} \\
& -\alpha \int_{\partial \Omega} \nu_{r}(\mathbf{y}) \mathcal{G}_{j k}^{\alpha}(\mathbf{y}-\mathbf{x}) h_{k}(\mathbf{y}) d \sigma_{y}, \quad j, r=1, \ldots, n,
\end{aligned}
$$

where $\partial_{j}:=\frac{\partial}{\partial x_{j}}$. We also employed the following integration by parts formula, which holds for any $p \in(1, \infty)($ cf. $[61,(2.16)])$

$$
\int_{\partial \Omega} f\left(\partial_{\tau_{j k}} g\right) d \sigma=\int_{\partial \Omega}\left(\partial_{\tau_{k j}} f\right) g d \sigma, \quad \forall f \in H_{p}^{1}(\partial \Omega), \forall g \in H_{p^{\prime}}^{1}(\partial \Omega)
$$

where $\frac{1}{p}+\frac{1}{p^{\prime}}=1$. The last integral in (3.58) follows from equations (3.4), which, in particular, yield that

$$
\left(\triangle_{y}-\alpha \mathbb{I}\right) \mathcal{G}^{\alpha}(\mathbf{y}-\mathbf{x})-\nabla_{y} \Pi(\mathbf{y}-\mathbf{x})=0, \quad \operatorname{div}_{y} \mathcal{G}^{\alpha}(\mathbf{y}-\mathbf{x})=0, \forall \mathbf{x} \in \mathbb{R}^{n} \backslash \partial \Omega, \mathbf{y} \in \partial \Omega
$$

In the case $\alpha=0$, formula (3.58) has been obtained in [61, (4.84)].

Now, from formula (3.58) and its counterpart corresponding to $\alpha=0$, we obtain for all $j, r=1, \ldots, n$,

$$
\partial_{r}\left(\mathbf{W}_{\alpha} \mathbf{h}\right)_{j}=\partial_{r}(\mathbf{W h})_{j}+\partial_{\ell}\left(\left(\mathbf{V}_{\alpha}-\mathbf{V}\right)\left(\partial_{\tau_{\ell r}} \mathbf{h}\right)\right)_{j}+\partial_{j}\left(\left(\mathbf{V}_{\alpha}-\mathbf{V}\right)\left(\partial_{\tau_{\ell r}} \mathbf{h}\right)\right)_{\ell}-\alpha\left(\mathbf{V}_{\alpha}\left(\nu_{r} \mathbf{h}\right)\right)_{j}, \quad \forall \mathbf{h} \in H_{p}^{1}\left(\partial \Omega, \mathbb{R}^{n}\right)
$$

Further, by using estimate (4.86) in [61, Proposition 4.2.8] for the Stokes double layer potential, Wh, property (3.38) for the Brinkman and Stokes single layer potentials involved in formula (3.61), and continuity of the tangential derivative operators $\partial_{\tau_{j k}}: H_{p}^{1}(\partial \Omega) \rightarrow L_{p}(\partial \Omega)$, we obtain inequality (3.57), as asserted (see also [38, (3.35)]).

Finally, inequalities (3.40), (3.56) and (3.57) imply inequality (3.41).

For any $n \geq 3$ and $\ell \geq 0$, there exists a constant $C=C(n, \ell, \alpha)>0$ such that the inequality (cf. [69, Theorem 2.4])

$$
\left|\nabla_{\times}^{\ell} \mathcal{G}^{\alpha}(\mathbf{x})\right| \leq \frac{C}{\left(1+\alpha|\mathbf{x}|^{2}\right)|\mathbf{x}|^{n-2+\ell}}
$$

holds and implies that $\left|\mathcal{G}^{\alpha}(\mathbf{x}-\mathbf{y})\right| \leq C_{0}|\mathbf{x}-\mathbf{y}|^{2-n}$, with some constant $C_{0}=C_{0}(n, \alpha)>0$. Then in view of [47, Proposition 1], for any $\mathbf{g} \in L_{p}\left(\partial \Omega, \mathbb{R}^{n}\right)$ there exist the non-tangential limits of the Brinkman single layer potential $\mathbf{V}_{\alpha} \mathbf{g}$ at almost all points of $\partial \Omega$. Moreover, the existence of the non-tangential limits of $\nabla \mathbf{V}_{\alpha} \mathbf{g}$ at almost all points of $\partial \Omega$ follows immediately from [69, Lemma 3.3]. For $\mathcal{Q}^{s} \mathbf{g}$ such a result is valid since the Brinkman pressure single layer potential coincides with the Stokes pressure single layer potential, for which the result is well known, cf., e.g., [61, Proposition 4.2.2] and [69, Lemma 3.3].

If $\mathbf{g} \in H_{p}^{-1}\left(\partial \Omega, \mathbb{R}^{n}\right)$ then the existence of the non-tangential limits of $\mathbf{V}_{\alpha} \mathbf{g}$ a.e. on $\partial \Omega$ follows from formula (3.47) and the corresponding statement for the existence of non-tangential limits for a single layer potential and the gradient a single layer potential with a density in $L_{p}\left(\partial \Omega, \mathbb{R}^{n}\right)$.

Now let $\mathbf{h} \in L_{p}\left(\partial \Omega, \mathbb{R}^{n}\right)$. Then the existence of the non-tangential limits of the Brinkman double layer potential $\mathbf{W}_{\alpha} \mathbf{h}$ at almost all points of $\partial \Omega$ follows easily from the case $\alpha=0$. Indeed, estimate (3.49) and [47, Proposition 1] imply that the difference

$$
\begin{aligned}
\left(\mathbf{W}_{\alpha} \mathbf{h}\right)_{j}(\mathbf{x})-(\mathbf{W h})_{j}(\mathbf{x}) & =\int_{\partial \Omega}\left(S_{i j k}^{\alpha}(\mathbf{y}-\mathbf{x})-S_{i j k}(\mathbf{y}-\mathbf{x})\right) \nu_{k}(\mathbf{y}) h_{i}(\mathbf{y}) d \sigma_{\mathbf{y}} \\
& =\int_{\partial \Omega}\left\{\left(\frac{\partial \mathcal{G}_{i j}^{\alpha}(\mathbf{y}-\mathbf{x})}{\partial y_{k}}-\frac{\partial \mathcal{G}_{i j}(\mathbf{y}-\mathbf{x})}{\partial y_{k}}\right)+\left(\frac{\partial \mathcal{G}_{k j}^{\alpha}(\mathbf{y}-\mathbf{x})}{\partial y_{i}}-\frac{\partial \mathcal{G}_{k j}(\mathbf{y}-\mathbf{x})}{\partial y_{i}}\right)\right\} \nu_{k}(\mathbf{y}) h_{i}(\mathbf{y}) d \sigma_{y}, \mathbf{x} \in \Omega_{ \pm}
\end{aligned}
$$


has non-tangential limits $\left(\left(\mathbf{W}_{\alpha} \mathbf{h}\right)_{j}-(\mathbf{W h})_{j}\right)_{n t}^{ \pm}\left(\mathbf{x}_{0}\right)$ at almost all points $\mathbf{x}_{0} \in \partial \Omega$. On the other hand, according to [61, Proposition 4.2.2] there exist the non-tangential limits of the Stokes double layer potential Wh at almost all points $\mathbf{x}_{0}$ of $\partial \Omega$. Therefore, the non-tangential limits of the Brinkman double layer potential $\mathbf{W}_{\alpha} \mathbf{h}$ exist as well at almost all points $\mathbf{x}$ of $\partial \Omega$.

Now let $\mathbf{h} \in H_{p}^{1}\left(\partial \Omega, \mathbb{R}^{n}\right)$. Then the existence of the non-tangential limits of $\nabla \mathbf{W}$. $\mathbf{h}$ at almost all points of $\partial \Omega$ follows from their existence in the case $\alpha=0$ (cf. [61, (4.91)]), formula (3.61), and the statement for the existence of non-tangential limits for a single layer potential and the gradient a single layer potential with a density in $L_{p}\left(\partial \Omega, \mathbb{R}^{n}\right)$, while the existence of non-tangential limits of $\mathcal{Q}_{\alpha} \mathbf{h}$ a.e. on $\partial \Omega$ is provided by the corresponding result in the case $\alpha=0$ (cf. [61, (4.85)]) and [47, Proposition 1] applied to the complementary term $\left(\mathcal{Q}_{\alpha}^{d}-\mathcal{Q}^{d}\right) \mathbf{h}=\alpha V_{\Delta}(\mathbf{h} \cdot \boldsymbol{\nu})$, which by (3.52) is the Laplace single layer potential with density $\alpha \mathbf{h} \cdot \boldsymbol{\nu} \in L_{p}(\partial \Omega)$.

Finally, note that inequalities (3.42)-(3.45) follow from inequalities (3.38)-(3.41) and the estimate $\left\|f_{\text {nt }}^{ \pm}\right\|_{L_{p}(\partial \Omega)} \leq\|M(f)\|_{L_{p}(\partial \Omega)}$. whenever $f$ has the property that both $f_{\mathrm{nt}}^{ \pm}$and $M(f)$ exist a.e on $\partial \Omega$ (see [16, Remark 9])

The mapping properties of layer potential operators for the Stokes system (i.e., for $\alpha=0$ ) in Bessel-potential and Besov spaces on bounded Lipschitz domains, as well as their jump relations across a Lipschitz boundary, are well known, cf., e.g., [23], [27], [61, Theorem 10.5.3], [62, Theorem 3.1, Proposition 3.3]. The main properties of layer potential operators for the Brinkman system are collected below (some of them are also available in [22, Proposition 3.4], [32, Lemma 3.4], [33, Lemma 3.1], [62, Theorem 3.1], [69, Theorems 3.4 and 3.5]).

Theorem 3.5 Let $\Omega_{+} \subset \mathbb{R}^{n}(n \geq 3)$ be a bounded Lipschitz domain with connected boundary $\partial \Omega$ and let $\Omega_{-}:=\mathbb{R}^{n} \backslash \bar{\Omega}_{+}$. Let $p, q \in(1, \infty), \alpha>0$, and $p^{*}:=\max \{p, 2\}$. Let $t \geq-\frac{1}{p^{\prime}}$ be arbitrary, where $\frac{1}{p}+\frac{1}{p^{\prime}}=1$

(i) Then the following operators are linear and continuous,

$$
\begin{aligned}
& \left.\mathbf{V}_{\alpha}\right|_{\Omega_{+}}: L_{p}\left(\partial \Omega, \mathbb{R}^{n}\right) \rightarrow B_{p, p^{*} ; \operatorname{div}}^{1+\frac{1}{p}}\left(\Omega_{+}, \mathbb{R}^{n}\right),\left.\mathcal{Q}^{s}\right|_{\Omega_{+}}: L_{p}\left(\partial \Omega, \mathbb{R}^{n}\right) \rightarrow B_{p, p^{*}}^{\frac{1}{p}}\left(\Omega_{+}\right), \\
& \left(\left.\mathbf{V}_{\alpha}\right|_{\Omega_{+}}, \mathcal{Q}^{s} \Omega_{\Omega_{+}}\right): L_{p}\left(\partial \Omega, \mathbb{R}^{n}\right) \rightarrow \mathfrak{B}_{p, p^{*} ; \operatorname{div}}^{1+\frac{1}{2}, t}\left(\Omega_{+}, \mathcal{L}_{\alpha}\right), \\
& \left.\mathbf{V}_{\alpha}\right|_{\Omega_{+}}: H_{p}^{-1}\left(\partial \Omega, \mathbb{R}^{n}\right) \rightarrow B_{p, p^{*} ; \operatorname{div}}^{\frac{1}{p}}\left(\Omega_{+}, \mathbb{R}^{n}\right),\left.\mathcal{Q}^{s}\right|_{\Omega_{+}}: H_{p}^{-1}\left(\partial \Omega, \mathbb{R}^{n}\right) \rightarrow B_{p, p^{*}}^{-1+\frac{1}{p}}\left(\Omega_{+}\right), \\
& \left(\left.\mathbf{V}_{\alpha}\right|_{\Omega_{+}},\left.\mathcal{Q}^{s}\right|_{\Omega_{+}}\right): H_{p}^{-1}\left(\partial \Omega, \mathbb{R}^{n}\right) \rightarrow \mathfrak{B}_{p, p^{*} ; \operatorname{div}}^{\frac{1}{p}, t}\left(\Omega_{+}, \mathcal{L}_{\alpha}\right), \\
& \left.\mathbf{W}_{\alpha}\right|_{\Omega_{+}}: H_{p}^{1}\left(\partial \Omega, \mathbb{R}^{n}\right) \rightarrow B_{p, p^{*} ; \operatorname{div}}^{1+\frac{1}{p}}\left(\Omega_{+}, \mathbb{R}^{n}\right),\left.\mathcal{Q}_{\alpha}^{d}\right|_{\Omega_{+}}: H_{p}^{1}\left(\partial \Omega, \mathbb{R}^{n}\right) \rightarrow B_{p, p^{*}}^{\frac{1}{p}}\left(\Omega_{+}\right), \\
& \left(\left.\mathbf{W}_{\alpha}\right|_{\Omega_{+}},\left.\mathcal{Q}_{\alpha}^{d}\right|_{\Omega_{+}}\right): H_{p}^{1}\left(\partial \Omega, \mathbb{R}^{n}\right) \rightarrow \mathfrak{B}_{p, p^{*} ; \operatorname{div}}^{1+\frac{1}{,}, t}\left(\Omega_{+}, \mathcal{L}_{\alpha}\right) . \\
& \left.\mathbf{W}_{\alpha}\right|_{\Omega_{+}}: L_{p}\left(\partial \Omega, \mathbb{R}^{n}\right) \rightarrow B_{p, p^{*} ; \operatorname{div}}^{\frac{1}{p}}\left(\Omega_{+}, \mathbb{R}^{n}\right),\left.\mathcal{Q}_{\alpha}^{d}\right|_{\Omega_{+}}: L_{p}\left(\partial \Omega, \mathbb{R}^{n}\right) \rightarrow B_{p, p^{*}}^{\frac{1}{p}-1}\left(\Omega_{+}\right), \\
& \left(\left.\mathbf{W}_{\alpha}\right|_{\Omega_{+}},\left.\mathcal{Q}_{\alpha}^{d}\right|_{\Omega_{+}}\right): L_{p}\left(\partial \Omega, \mathbb{R}^{n}\right) \rightarrow \mathfrak{B}_{p, p^{*} ; \operatorname{div}}^{\frac{1}{p}, t}\left(\Omega_{+}, \mathcal{L}_{\alpha}\right) .
\end{aligned}
$$

(ii) Moreover, the following operators are also linear and continuous for $s \in(0,1)$,

$$
\begin{aligned}
& \mathbf{V}_{\alpha}: B_{p, q}^{s-1}\left(\partial \Omega, \mathbb{R}^{n}\right) \rightarrow B_{p, q \text {, div }}^{s+\frac{1}{p}}\left(\mathbb{R}^{n}, \mathbb{R}^{n}\right), \mathcal{Q}^{s}: B_{p, q}^{s-1}\left(\partial \Omega, \mathbb{R}^{n}\right) \rightarrow B_{p, q ; \mathrm{loc}}^{s+\frac{1}{p}-1}\left(\mathbb{R}^{n}\right), \\
& \left.\mathbf{V}_{\alpha}\right|_{\Omega_{+}}: B_{p, q}^{s-1}\left(\partial \Omega, \mathbb{R}^{n}\right) \rightarrow B_{p, q \text {, div }}^{s+\frac{1}{p}}\left(\Omega_{+}, \mathbb{R}^{n}\right),\left.\left(\mathcal{Q}^{s}\right)\right|_{\Omega_{+}}: B_{p, q}^{s-1}\left(\partial \Omega, \mathbb{R}^{n}\right) \rightarrow B_{p, q}^{s+\frac{1}{p}-1}\left(\Omega_{+}\right) \\
& \left(\left.\mathbf{V}_{\alpha}\right|_{\Omega_{+}},\left.\mathcal{Q}^{s}\right|_{\Omega_{+}}\right): B_{p, q}^{s-1}\left(\partial \Omega, \mathbb{R}^{n}\right) \rightarrow \mathfrak{B}_{p, q, \text { div }}^{s+\frac{1}{p}, t}\left(\Omega_{+}, \mathcal{L}_{\alpha}\right), \\
& \left.\mathbf{W}_{\alpha}\right|_{\Omega_{+}}: B_{p, q}^{s}\left(\partial \Omega, \mathbb{R}^{n}\right) \rightarrow B_{p, q ; \operatorname{div}}^{s+\frac{1}{p}}\left(\Omega_{+}, \mathbb{R}^{n}\right),\left.\mathcal{Q}_{\alpha}^{d}\right|_{\Omega_{+}}: B_{p, q}^{s}\left(\partial \Omega, \mathbb{R}^{n}\right) \rightarrow B_{p, q}^{s+\frac{1}{p}-1}\left(\Omega_{+}\right), \\
& \left(\left.\mathbf{W}_{\alpha}\right|_{\Omega_{+}},\left.\mathcal{Q}_{\alpha}^{d}\right|_{\Omega_{+}}\right): B_{p, q}^{s}\left(\partial \Omega, \mathbb{R}^{n}\right) \rightarrow \mathfrak{B}_{p, q, \text { div }}^{s+\frac{1}{p}, t}\left(\Omega_{+}, \mathcal{L}_{\alpha}\right), \\
& \left.\mathbf{V}_{\alpha}\right|_{\Omega_{-}}: B_{p, q}^{s-1}\left(\partial \Omega, \mathbb{R}^{n}\right) \rightarrow B_{p, q ; \operatorname{div}}^{s+\frac{1}{p}}\left(\Omega_{-}, \mathbb{R}^{n}\right),\left.\mathcal{Q}^{s}\right|_{\Omega_{-}}: B_{p, q}^{s-1}\left(\partial \Omega, \mathbb{R}^{n}\right) \rightarrow B_{p, q ; \mathrm{loc}}^{s+\frac{1}{p}-1}\left(\bar{\Omega}_{-}\right), \\
& \left(\left.\mathbf{V}_{\alpha}\right|_{\Omega_{-}},\left.\mathcal{Q}^{s}\right|_{\Omega_{-}}\right): B_{p, q}^{s-1}\left(\partial \Omega, \mathbb{R}^{n}\right) \rightarrow \mathfrak{B}_{p, q ; \mathrm{div} ; \text { loc }}^{s+\frac{1}{p}, t}\left(\bar{\Omega}_{-}, \mathcal{L}_{\alpha}\right) \\
& \left.\mathbf{W}_{\alpha}\right|_{\Omega_{-}}: B_{p, q}^{s}\left(\partial \Omega, \mathbb{R}^{n}\right) \rightarrow B_{p, q ; \text { div } ; \text { loc }}^{s+\frac{1}{p}}\left(\bar{\Omega}_{-}, \mathbb{R}^{n}\right),\left.\mathcal{Q}^{d}\right|_{\Omega_{-}}: B_{p, q}^{s}\left(\partial \Omega, \mathbb{R}^{n}\right) \rightarrow B_{p, q ; \text { loc }}^{s+\frac{1}{p}-1}\left(\bar{\Omega}_{-}\right), \\
& \left(\left.\mathbf{W}_{\alpha}\right|_{\Omega_{-}},\left.\mathcal{Q}_{\alpha}^{d}\right|_{\Omega_{-}}\right): B_{p, q}^{s}\left(\partial \Omega, \mathbb{R}^{n}\right) \rightarrow \mathfrak{B}_{p, q, \text { div } ; \text { loc }}^{s+\frac{1}{2}, t}\left(\bar{\Omega}_{-}, \mathcal{L}_{\alpha}\right) .
\end{aligned}
$$

(iii) The following relations hold a.e. on $\partial \Omega$,

$$
\begin{aligned}
& \left(\mathbf{V}_{\alpha} \mathbf{g}\right)_{\mathrm{nt}}^{+}=\left(\mathbf{V}_{\alpha} \mathbf{g}\right)_{\mathrm{nt}}^{-}=: \mathcal{V}_{\alpha} \mathbf{g}, \quad \forall \mathbf{g} \in H_{p}^{-1}\left(\partial \Omega, \mathbb{R}^{n}\right) \\
& \frac{1}{2} \mathbf{h}+\left(\mathbf{W}_{\alpha} \mathbf{h}\right)_{\mathrm{nt}}^{+}=-\frac{1}{2} \mathbf{h}+\left(\mathbf{W}_{\alpha} \mathbf{h}\right)_{\mathrm{nt}}^{-}=: \mathbf{K}_{\alpha} \mathbf{h}, \quad \forall \mathbf{h} \in L_{p}\left(\partial \Omega, \mathbb{R}^{n}\right)
\end{aligned}
$$




$$
\begin{gathered}
-\frac{1}{2} \mathbf{g}+\mathbf{t}_{\mathrm{nt}}^{+}\left(\mathbf{V}_{\alpha} \mathbf{g}, \mathcal{Q}^{s} \mathbf{g}\right)=\frac{1}{2} \mathbf{g}+\mathbf{t}_{\mathrm{nt}}^{-}\left(\mathbf{V}_{\alpha} \mathbf{g}, \mathcal{Q}^{s} \mathbf{g}\right)=: \mathbf{K}_{\alpha}^{*} \mathbf{g}, \quad \forall \mathbf{g} \in L_{p}\left(\partial \Omega, \mathbb{R}^{n}\right) \\
\mathbf{t}_{\mathrm{nt}}^{+}\left(\mathbf{W}_{\alpha} \mathbf{h}, \mathcal{Q}_{\alpha}^{d} \mathbf{h}\right)=\mathbf{t}_{\mathrm{nt}}^{-}\left(\mathbf{W}_{\alpha} \mathbf{h}, \mathcal{Q}_{\alpha}^{d} \mathbf{h}\right)=: \mathbf{D}_{\alpha} \mathbf{h}, \quad \forall \mathbf{h} \in H_{p}^{1}\left(\partial \Omega, \mathbb{R}^{n}\right) ;
\end{gathered}
$$

where $\mathbf{K}_{\alpha}^{*}$ is the transpose of $\mathbf{K}_{\alpha ; \partial \Omega}$, and the following boundary integral operators are linear and bounded,

$$
\begin{aligned}
& \mathcal{V}_{\alpha}: L_{p}\left(\partial \Omega, \mathbb{R}^{n}\right) \rightarrow H_{p}^{1}\left(\partial \Omega, \mathbb{R}^{n}\right), \mathbf{K}_{\alpha}: H_{p}^{1}\left(\partial \Omega, \mathbb{R}^{n}\right) \rightarrow H_{p}^{1}\left(\partial \Omega, \mathbb{R}^{n}\right), \\
& \mathcal{V}_{\alpha}: H_{p}^{-1}\left(\partial \Omega, \mathbb{R}^{n}\right) \rightarrow L_{p}\left(\partial \Omega, \mathbb{R}^{n}\right), \mathbf{K}_{\alpha}: L_{p}\left(\partial \Omega, \mathbb{R}^{n}\right) \rightarrow L_{p}\left(\partial \Omega, \mathbb{R}^{n}\right), \\
& \mathbf{K}_{\alpha}^{*}: L_{p}\left(\partial \Omega, \mathbb{R}^{n}\right) \rightarrow L_{p}\left(\partial \Omega, \mathbb{R}^{n}\right), \mathbf{D}_{\alpha}: H_{p}^{1}\left(\partial \Omega, \mathbb{R}^{n}\right) \rightarrow L_{p}\left(\partial \Omega, \mathbb{R}^{n}\right) .
\end{aligned}
$$

For $\mathbf{h} \in B_{p, q}^{s}\left(\partial \Omega, \mathbb{R}^{n}\right)$ and $\mathbf{g} \in B_{p, q}^{s-1}\left(\partial \Omega, \mathbb{R}^{n}\right), s \in(0,1)$, the following relations hold a.e. on $\partial \Omega$,

$$
\begin{aligned}
& \gamma_{+}\left(\mathbf{V}_{\alpha} \mathbf{g}\right)=\gamma_{-}\left(\mathbf{V}_{\alpha} \mathbf{g}\right)=: \mathcal{V}_{\alpha} \mathbf{g}, \\
& \frac{1}{2} \mathbf{h}+\gamma_{+}\left(\mathbf{W}_{\alpha} \mathbf{h}\right)=-\frac{1}{2} \mathbf{h}+\gamma_{-}\left(\mathbf{W}_{\alpha} \mathbf{h}\right)=: \mathbf{K}_{\alpha} \mathbf{h}, \\
& -\frac{1}{2} \mathbf{g}+\mathbf{t}_{\alpha}^{+}\left(\mathbf{V}_{\alpha} \mathbf{g}, \mathcal{Q}^{s} \mathbf{g}\right)=\frac{1}{2} \mathbf{g}+\mathbf{t}_{\alpha}^{-}\left(\mathbf{V}_{\alpha} \mathbf{g}, \mathcal{Q}_{\partial \Omega}^{s} \mathbf{g}\right)=: \mathbf{K}_{\alpha}^{*} \mathbf{g}, \\
& \left.\mathbf{t}_{\alpha}^{+}\left(\mathbf{W}_{\alpha} \mathbf{h}, \mathcal{Q}_{\alpha}^{d} \mathbf{h}\right)=\mathbf{t}_{\alpha}^{-}(\mathbf{W})_{\alpha} \mathbf{h}, \mathcal{Q}_{\alpha ; \partial \Omega}^{d} \mathbf{h}\right)=: \mathbf{D}_{\alpha} \mathbf{h},
\end{aligned}
$$

and the following operators are linear and continuous,

$$
\begin{aligned}
& \mathcal{V}_{\alpha}: B_{p, q}^{s-1}\left(\partial \Omega, \mathbb{R}^{n}\right) \rightarrow B_{p, q}^{s}\left(\partial \Omega, \mathbb{R}^{n}\right), \mathbf{K}_{\alpha}: B_{p, q}^{s}\left(\partial \Omega, \mathbb{R}^{n}\right) \rightarrow B_{p, q}^{s}\left(\partial \Omega, \mathbb{R}^{n}\right) \\
& \mathbf{K}_{\alpha}^{*}: B_{p, q}^{s-1}\left(\partial \Omega, \mathbb{R}^{n}\right) \rightarrow B_{p, q}^{s-1}\left(\partial \Omega, \mathbb{R}^{n}\right), \quad \mathbf{D}_{\alpha}: B_{p, q}^{s}\left(\partial \Omega, \mathbb{R}^{n}\right) \rightarrow B_{p, q}^{s-1}\left(\partial \Omega, \mathbb{R}^{n}\right) .
\end{aligned}
$$

Proof. (i) First of all, we remark that all range spaces of the velocity vector-valued layer potential operators in (3.64)-(3.80) are divergence-free due to the second relations in (3.35)-(3.36). Further, let us note that by (3.33) and (3.8) the single layer potential can be presented as (cf. [18, (4.1)]),

$$
\mathbf{V}_{\alpha} \mathbf{g}=\left\langle\gamma \mathcal{G}^{\alpha}(\mathbf{x}, \cdot), \mathbf{g}\right\rangle_{\partial \Omega}=\left\langle\mathcal{G}^{\alpha}(\mathbf{x}, \cdot), \gamma^{\prime} \mathbf{g}\right\rangle_{\mathbb{R}^{n}}=\mathbf{N}_{\alpha ; \mathbb{R}^{n}} \circ \gamma^{\prime} \mathbf{g}
$$

for any $\mathbf{g} \in B_{p, q}^{s-1}\left(\partial \Omega, \mathbb{R}^{n}\right), p, q \in(1, \infty)$ and $s \in(0,1)$. Here the operator $\gamma^{\prime}: B_{p, q}^{s-1}\left(\partial \Omega, \mathbb{R}^{n}\right) \rightarrow B_{p, q ; \text { comp }}^{s-1-\frac{1}{\rho^{\prime}}}\left(\mathbb{R}^{n}, \mathbb{R}^{n}\right)$ is adjoint to the trace operator $\gamma: B_{p^{\prime}, q^{\prime} ; \text { loc }}^{1-s+\frac{1}{p^{\prime}}}\left(\mathbb{R}^{n}, \mathbb{R}^{n}\right) \rightarrow B_{p^{\prime}, q^{\prime}}^{1-s}\left(\partial \Omega, \mathbb{R}^{n}\right)$ and they both are continues due to Lemma 2.4.

Next, we show the continuity of the first operator in (3.64) in the case $\alpha>0$ (i.e., for the Brinkman system). To this end, we split the Brinkman single-layer potential operator into two operators, as $\mathbf{V}_{\alpha}=\mathbf{V}+\mathbf{V}_{\alpha ; 0}$, where $\mathbf{V}_{\alpha ; 0}$ is the complementary single-layer potential operator, i.e.,

$$
\mathbf{V}_{\alpha ; 0}:=\mathbf{V}_{\alpha}-\mathbf{V}=\mathbf{N}_{\alpha ; 0 ; \mathbb{R}^{n}} \circ \gamma^{\prime} \circ \iota
$$

where the imbedding operator $\iota: L_{p}\left(\partial \Omega, \mathbb{R}^{n}\right) \hookrightarrow B_{p, p^{*}}^{s-1}\left(\partial \Omega, \mathbb{R}^{n}\right)$ is continuous for any $s \in(0,1)$ and $p \in(1, \infty)$. In addition, $\mathbf{N}_{\alpha ; 0 ; \mathbb{R}^{n}}:=\mathbf{N}_{\alpha ; \mathbb{R}^{n}}-\mathbf{N}_{0 ; \mathbb{R}^{n}}$ is a pseudodifferential operator of order -4 with the kernel $\mathcal{G}^{\alpha ; 0}:=\mathcal{G}^{\alpha}-\mathcal{G}$ (see formula (2.27) in [33]), and hence the linear operator

$$
\mathbf{N}_{\alpha ; 0 ; \mathbb{R}^{n}}: B_{p, p^{*} ; \text { comp }}^{s-1-\frac{1}{p^{\prime}}}\left(\mathbb{R}^{n}, \mathbb{R}^{n}\right) \rightarrow B_{p, p^{*} ; \text { loc }}^{s+3-\frac{1}{p^{\prime}}}\left(\mathbb{R}^{n}, \mathbb{R}^{n}\right)
$$

is continuous for any $s \in(0,1)$ and $p \in(1, \infty)$, where $\frac{1}{p^{\prime}}=1-\frac{1}{p}$, and $B_{p, p^{*} ; \text { comp }}^{s-1-\frac{1}{p^{\prime}}}\left(\mathbb{R}^{n}\right.$, $\left.\mathbb{R}^{n}\right)$ is the space of distributions in $B_{p, p^{*}}^{s-1-\frac{1}{p^{\prime}}}\left(\mathbb{R}^{n}, \mathbb{R}^{n}\right)$ with compact supports. Then formula (3.95) and the continuity of the involved operators imply that the operators

$$
\mathbf{V}_{\alpha ; 0}: L_{p}\left(\partial \Omega, \mathbb{R}^{n}\right) \rightarrow B_{p, p^{*} ; \text { loc }}^{s+2+\frac{1}{p}}\left(\mathbb{R}^{n}, \mathbb{R}^{n}\right),\left.\left(\mathbf{V}_{\alpha ; 0}\right)\right|_{\Omega_{+}}: L_{p}\left(\partial \Omega, \mathbb{R}^{n}\right) \rightarrow B_{p, p^{*}}^{s+2+\frac{1}{p}}\left(\Omega_{+}, \mathbb{R}^{n}\right)
$$

are continuous as well. Now, the continuity of the embedding $B_{p, p^{*}}^{s+2+\frac{1}{p}}\left(\Omega_{+}, \mathbb{R}^{n}\right) \hookrightarrow B_{p, p^{*}}^{1+\frac{1}{p}}\left(\Omega_{+}, \mathbb{R}^{n}\right)$ for any $s \in(0,1)$ shows that

$$
\mathbf{V}_{\alpha ; 0}: L_{p}\left(\partial \Omega, \mathbb{R}^{n}\right) \rightarrow B_{p, p^{*}}^{1+\frac{1}{p}}\left(\Omega_{+}, \mathbb{R}^{n}\right)
$$

is a continuous operator, even compact.

Moreover, the Stokes single layer potential operator $\mathbf{V}: L_{p}\left(\partial \Omega, \mathbb{R}^{n}\right) \rightarrow L_{p}\left(\Omega_{+}, \mathbb{R}^{n}\right)$ is continuous (cf., e.g., the mapping property $(10.73)$ in [61] and the continuity of the embeddings $L_{p}\left(\partial \Omega, \mathbb{R}^{n}\right) \hookrightarrow B_{p, p^{*}}^{s-1}\left(\partial \Omega, \mathbb{R}^{n}\right)$ and $B_{p, p^{*}}^{s+\frac{1}{p}}\left(\Omega_{+}, \mathbb{R}^{n}\right) \hookrightarrow L_{p}\left(\Omega_{+}, \mathbb{R}^{n}\right)$ for any $s \in(0,1))$. 
On the other hand, the kernel $\nabla \mathcal{G}$ of the integral operator $\nabla \mathbf{V}$ satisfies the relations

$$
\nabla \mathcal{G} \in C^{\infty}\left(\mathbb{R}^{n} \backslash\{0\}\right),(\nabla \mathcal{G})(-\mathbf{x})=-(\nabla \mathcal{G})(\mathbf{x}), \quad(\nabla \mathcal{G})(\lambda \mathbf{x})=\lambda^{-(n-1)}(\nabla \mathcal{G})(\mathbf{x}), \quad \forall \lambda>0 .
$$

Then, in view of [58, Proposition 2.68], there exists a constant $C_{0} \equiv C_{0}\left(\Omega_{+}, p\right)>0$ such that

$$
\|\nabla \mathbf{V} \mathbf{g}\|_{B_{p, p^{*}}^{\frac{1}{p}}\left(\Omega_{+}, \mathbb{R}^{n \times n)}\right.} \leq C_{0}\|\mathbf{g}\|_{L_{p}\left(\partial \Omega, \mathbb{R}^{n}\right)}, \quad \forall \mathbf{g} \in L_{p}\left(\partial \Omega, \mathbb{R}^{n}\right)
$$

Consequently, there exists a constant $\mathfrak{C} \equiv \mathfrak{C}\left(\Omega_{+}, p\right)>0$ such that

$$
\|\mathbf{V g}\|_{B_{p, p^{*}}^{1+\frac{1}{p}}\left(\Omega_{+}, \mathbb{R}^{n}\right)}=\|\mathbf{V} \mathbf{g}\|_{L_{p}\left(\Omega_{+}, \mathbb{R}^{n}\right)}+\|\nabla \mathbf{V} \mathbf{g}\|_{B_{p, p^{*}}^{\frac{1}{p}}\left(\Omega_{+}, \mathbb{R}^{n \times n}\right)} \leq \mathfrak{C}\|\mathbf{g}\|_{L_{p}\left(\Omega_{+}, \mathbb{R}^{n}\right)}, \forall \mathbf{g} \in L_{p}\left(\Omega_{+}, \mathbb{R}^{n}\right),
$$

which shows that the Stokes single layer potential operator

$$
\mathbf{V}: L_{p}\left(\partial \Omega, \mathbb{R}^{n}\right) \rightarrow B_{p, p^{*}}^{1+\frac{1}{p}}\left(\Omega_{+}, \mathbb{R}^{n}\right)
$$

is also continuous (cf., e.g., [62, Theorem 7.1, (3.33)], see also [23] for $p=2$ ). This mapping property and the continuity of operator (3.97) show that the Brinkman single layer operator $\mathbf{V}_{\alpha}: L_{p}\left(\partial \Omega, \mathbb{R}^{n}\right) \rightarrow B_{p, p^{*}}^{1+\frac{1}{p}}\left(\Omega_{+}, \mathbb{R}^{n}\right)$ is continuous, as well.

Let us show the continuity of the second operator in (3.64). To this end, we note that the Stokes single layer pressure potential $\mathcal{Q}^{s} \mathbf{f}$ with a density $\mathbf{f}=\left(f_{1}, \ldots, f_{n}\right) \in L_{p}\left(\partial \Omega, \mathbb{R}^{n}\right)$ can be written as

$$
\left(\mathcal{Q}^{s} \mathbf{f}\right)(\mathbf{x})=\left(\operatorname{div} V_{\triangle} \mathbf{f}\right)(\mathbf{x}), \quad \forall \mathbf{x} \in \mathbb{R}^{n} \backslash \partial \Omega
$$

where $V_{\triangle} g$ is the harmonic single layer potential with density $g \in L_{p}(\partial \Omega)$, given by

$$
\left(V_{\triangle} g\right)(\mathbf{x}):=-\frac{1}{(n-2) \tilde{\omega}_{n}} \int_{\partial \Omega} \frac{1}{|\mathbf{x}-\mathbf{y}|^{n-2}} g(\mathbf{y}) d \sigma_{y}, \quad \mathbf{x} \in \mathbb{R}^{n} \backslash \partial \Omega .
$$

Then the continuity of the single layer pressure potential potential operator $\mathcal{Q}^{s}: L_{p}\left(\partial \Omega, \mathbb{R}^{n}\right) \rightarrow B_{p, p^{*}}^{\frac{1}{p}}\left(\Omega_{+}\right)$for any $p \in(1, \infty)$ is a direct consequence of Proposition 4.23 in [59]. Note that Proposition 2.68 in [58] applies as well, and shows the desired continuity of the single layer pressure potential operator in (3.64) (see also [62, Theorem 3.1, (3.30)]). Thus, we have proved the continuity of the operators in (3.64).

Continuity of the first operator in (3.66) follows from the continuity of operators involved in the right hand side of equality (3.47). Continuity of the second operator in (3.66) follows from equality (3.102), which is valid also for any $\mathbf{f} \in H_{p}^{-1}\left(\partial \Omega\right.$, $\left.\mathbb{R}^{n}\right)$, and by the continuity of the harmonic single layer potential operator $V_{\triangle}$ from $H_{p}^{-1}(\partial \Omega)$ to $B_{p, p^{*}}^{\frac{1}{p}}\left(\Omega_{+}\right)$. Indeed, for any $f \in H_{p}^{-1}(\partial \Omega)$ there exist $f_{0}, f_{r \ell} \in L_{p}(\partial \Omega), r, l=1, \ldots n$, such that $f=f_{0}+\sum_{r, \ell=1}^{n} \partial_{\tau_{r \ell}} f_{r \ell}$ (see (3.46)). Then by using the integration by parts formula (3.59), we obtain that

$$
\left(V_{\triangle} f\right)(\mathbf{x})=\int_{\partial \Omega} \mathcal{G}_{\triangle}(\mathbf{x}-\mathbf{y}) f_{0}(\mathbf{y}) d \sigma_{\mathrm{y}}-\sum_{r, \ell=1}^{n} \int_{\partial \Omega}\left(\partial_{\tau_{r s, y}} \mathcal{G}_{\triangle}(\mathbf{x}, \mathbf{y})\right) f_{r \ell}(\mathbf{y}) d \sigma_{\mathrm{y}}, \forall \mathbf{x} \in \mathbb{R}^{n} \backslash \partial \Omega,
$$

where $\mathcal{G}_{\triangle}(\mathbf{x}, \mathbf{y})$ is the fundamental solution of the Laplace equation in $\mathbb{R}^{n}$ ( $n \geq 3$ ). By using again [58, Proposition 2.68] (see also (3.99)) and the continuity of the Laplace single layer potential operator $V_{\Delta}: L_{p}(\partial \Omega) \rightarrow B_{p, p^{*}}^{1+\frac{1}{p}}\left(\Omega_{+}\right)$(see, e.g., [59, Proposition 4.23] and property (3.49) in [62, Proposition 3.3]), there exists a constant $C_{0}$ such that

$$
\left\|V_{\triangle} f\right\|_{B_{p, p^{*}}^{1+\frac{1}{p}}\left(\Omega_{+}\right)}=\left\|V_{\triangle} f\right\|_{L_{p}\left(\Omega_{+}\right)}+\left\|\nabla V_{\triangle} f\right\|_{\left.B_{p, p^{*}}^{\frac{1}{p}} \Omega_{+}, \mathbb{R}^{n}\right)} \leq C_{0}\|f\|_{L_{p}\left(\Omega_{+}\right)}, \forall f \in L_{p}\left(\Omega_{+}\right)
$$

Thus, the operator $\nabla V_{\triangle}: L_{p}(\partial \Omega) \rightarrow B_{p, p^{*}}^{\frac{1}{p}}\left(\Omega_{+}, \mathbb{R}^{n}\right)$ is also continuous. Finally, by continuity of this operator and of the operator $V_{\triangle}: L_{p}(\partial \Omega) \rightarrow B_{p, p^{*}}^{1+\frac{1}{p}}\left(\Omega_{+}\right)$and also by the second relation in (3.46), we obtain from (3.104) continuity of the operator $V_{\triangle}: H_{p}^{-1}(\partial \Omega) \rightarrow B_{p, p^{*}}^{\frac{1}{p}}\left(\Omega_{+}\right)$and, accordingly, continuity of the second operator in (3.66).

Let us now show the continuity of the first operator in (3.68). To this end, we notice that the Brinkman double-layer potential operator can be written as $\mathbf{W}_{\alpha}=\mathbf{W}+\mathbf{W}_{\alpha ; 0}$, where $\mathbf{W}_{\alpha ; 0}$ is the complementary double layer potential operator, i.e.

$$
\mathbf{W}_{\alpha ; 0}:=\mathbf{W}_{\alpha}-\mathbf{W}=\mathbb{K}_{\alpha ; 0} \circ \gamma^{\prime} \circ \mathfrak{N}
$$

(see [33, Eq. (3.31)]), where the operator $\mathfrak{N}: H_{p}^{1}\left(\partial \Omega, \mathbb{R}^{n}\right) \rightarrow L_{p}\left(\partial \Omega, \mathbb{R}^{n} \otimes \mathbb{R}^{n}\right) \hookrightarrow B_{p, p^{*}}^{-s}\left(\partial \Omega, \mathbb{R}^{n} \otimes \mathbb{R}^{n}\right), \mathfrak{N h}(x):=\boldsymbol{\nu}(x) \otimes \mathbf{h}(x)$, is continuous for any $s \in(0,1)$. In addition, $\mathbb{K}_{\alpha ; 0}$ is a pseudodifferential operator of order -3 with the kernel $\mathbf{S}^{\alpha ; 0}:=\mathbf{S}^{\alpha}-\mathbf{S}$ (cf., e.g., $[33,(2.27)])$, and hence the operator

$$
\mathbb{K}_{\alpha ; 0}: B_{p, p^{*} ; \text { comp }}^{-1-s+\frac{1}{p}}\left(\mathbb{R}^{n}, \mathbb{R}^{n} \otimes \mathbb{R}^{n}\right) \rightarrow B_{p, p^{*} ; \text { loc }}^{2-s+\frac{1}{p}}\left(\mathbb{R}^{n}, \mathbb{R}^{n}\right)
$$




$$
\left(\mathbb{K}_{\alpha ; 0} \mathbb{T}\right)_{j}(\mathbf{x}):=\left\langle\left(S_{j i \ell}^{\alpha}-S_{j i \ell}\right)(\cdot, \mathbf{x}), T_{i \ell}\right\rangle_{\mathbb{R}^{n}}, \forall \mathbb{T} \in B_{p, p^{*} ; \text { comp }}^{-1-s+\frac{1}{p}}\left(\mathbb{R}^{n}, \mathbb{R}^{n} \otimes \mathbb{R}^{n}\right),
$$

is also linear and continuous for any $s \in(0,1)$, where $B_{p, p^{*} ; \text { comp }}^{-1-s+\frac{1}{p}}\left(\mathbb{R}^{n}, \mathbb{R}^{n} \otimes \mathbb{R}^{n}\right)$ is the space of all distributions in $B_{p, p^{*}}^{-1-s+\frac{1}{p}}\left(\mathbb{R}^{n}, \mathbb{R}^{n} \otimes\right.$ $\mathbb{R}^{n}$ ) having compact support in $\mathbb{R}^{n}$. In addition, the trace operator $\gamma: B_{p^{\prime}, p^{*} ; \text { loc }}^{s+\frac{1}{p^{\prime}}}\left(\mathbb{R}^{n} \otimes \mathbb{R}^{n}\right) \rightarrow B_{p^{\prime}, p^{* \prime}}^{s}\left(\partial \Omega, \mathbb{R}^{n} \otimes \mathbb{R}^{n}\right)($ acting on matrix valued functions) and its adjoint $\gamma^{\prime}: B_{p, p^{*}}^{-s}\left(\partial \Omega, \mathbb{R}^{n} \otimes \mathbb{R}^{n}\right) \rightarrow B_{p, p^{*} ; \text { comp }}^{-s-1+\frac{1}{p}}\left(\mathbb{R}^{n}, \mathbb{R}^{n} \otimes \mathbb{R}^{n}\right)$ are continuous (see the proof of $[18$, Theorem 1]). Then formula (3.106) and the continuity of the involved operators imply that the operators

$$
\mathbf{W}_{\alpha ; 0}: H_{p}^{1}\left(\partial \Omega, \mathbb{R}^{n}\right) \rightarrow B_{p, p^{*} ; \text { loc }}^{2-s+\frac{1}{p}}\left(\mathbb{R}^{n}, \mathbb{R}^{n}\right),\left.\left(\mathbf{W}_{\alpha ; 0}\right)\right|_{\Omega_{+}}: H_{p}^{1}\left(\partial \Omega, \mathbb{R}^{n}\right) \rightarrow B_{p, p^{*}}^{2-s+\frac{1}{p}}\left(\Omega_{+}, \mathbb{R}^{n}\right)
$$

are continuous as well. Now, the continuity of the embedding $B_{p, p^{*}}^{2+\frac{1}{p}-s}\left(\Omega_{+}, \mathbb{R}^{n}\right) \hookrightarrow B_{p, p^{*}}^{1+\frac{1}{p}}\left(\Omega_{+}, \mathbb{R}^{n}\right)$ for any $s \in(0,1)$ shows that

$$
\mathbf{W}_{\alpha ; 0}: H_{p}^{1}\left(\partial \Omega, \mathbb{R}^{n}\right) \rightarrow B_{p, p^{*}}^{1+\frac{1}{p}}\left(\Omega_{+}, \mathbb{R}^{n}\right)
$$

is a continuous operator, even compact. Let us now show that the Stokes double-layer potential operator

$$
\mathbf{W}: H_{p}^{1}\left(\partial \Omega, \mathbb{R}^{n}\right) \rightarrow B_{p, p^{*}}^{1+\frac{1}{p}}\left(\Omega_{+}, \mathbb{R}^{n}\right)
$$

is continuous as well. In the setting of Riemannian manifolds and for double layer potentials for second order elliptic equations, this continuity property follows from [63, Theorem 8.5], but we will provide a direct proof here in the context of Euclidean setting. To this end, we use the following characterization of the space $H_{p}^{1}(\partial \Omega)$

$$
h \in H_{p}^{1}(\partial \Omega) \Longleftrightarrow h \in L_{p}(\partial \Omega), \partial_{\tau_{j k}} h \in L_{p}(\partial \Omega), j, k=1, \ldots, n
$$

(cf., e.g., $[61,(2.11)]$ ), and recall that the tangential derivative operators $\partial_{\tau_{j k}}: H_{p}^{1}(\partial \Omega) \rightarrow L_{p}(\partial \Omega)$ are continuous. In addition, consider the operator $V_{j k}$ defined as

$$
\left(V_{j k} g\right)(\mathbf{x}):=\int_{\partial \Omega} \mathcal{G}_{j k}(\mathbf{x}-\mathbf{y}) g(\mathbf{y}) d \sigma_{y}, \quad \mathbf{x} \in \mathbb{R}^{n} \backslash \partial \Omega
$$

We have proved that the Stokes single layer potential operator $(3.101)$ is continuous for any $p \in(1, \infty)$ (see also [62, Theorem 3.1, (3.33)]). Consequently, the operators

$$
V_{j k}: L_{p}(\partial \Omega) \rightarrow B_{p, p^{*}}^{1+\frac{1}{p}}\left(\Omega_{+}\right)
$$

are continuous as well, for all $j, k=1, \ldots, n$. Recall that the operator $V_{\Delta}: L_{p}(\partial \Omega) \rightarrow B_{p, p^{*}}^{1+\frac{1}{p}}\left(\Omega_{+}\right)$is also linear and continuous. Finally, we mention the following formula (cf. [61, (4.84)])

$$
\partial_{r}(\mathbf{W h})_{j}=-\partial_{\ell} V_{j k}\left(\partial_{\tau_{\ell r}} h_{k}\right)-\partial_{j} V_{\ell k}\left(\partial_{\tau_{\ell r}} h_{k}\right)-\partial_{k} V_{\Delta}\left(\partial_{\tau_{j r}} h_{k}\right) \text { in } \mathbb{R}^{n} \backslash \partial \Omega
$$

which holds for every $\mathbf{h} \in H_{p}^{1}\left(\partial \Omega, \mathbb{R}^{n}\right)$ and $j, r=1, \ldots, n$, where $h_{j}$ is the $j$-th component of $\mathbf{h}$. Then by using the continuity of operator (3.112) and properties (3.110) and (3.113), we deduce that the operators

$$
\partial_{r}(\mathbf{W})_{j}: H_{p}^{1}\left(\partial \Omega, \mathbb{R}^{n}\right) \rightarrow B_{p, p^{*}}^{\frac{1}{\bar{p}}}\left(\Omega_{+}\right), \quad r, j=1, \ldots, n
$$

are continuous. By [61, Proposition 10.5.1, (10.68)], the operator $\mathbf{W}: H_{p}^{1}\left(\partial \Omega, \mathbb{R}^{n}\right) \rightarrow L_{p}\left(\Omega_{+}, \mathbb{R}^{n}\right)$ is also continuous (as its range is a subspace of the space $H_{p}^{s+\frac{1}{p}}\left(\Omega_{+}, \mathbb{R}^{n}\right)$ for any $s \in(0,1), H_{p}^{1}\left(\partial \Omega, \mathbb{R}^{n}\right) \hookrightarrow B_{p, p}^{s}\left(\partial \Omega, \mathbb{R}^{n}\right)$ (due to formula (A.12)), and $\left.B_{p, p}^{s+\frac{1}{p}}\left(\Omega_{+}, \mathbb{R}^{n}\right) \hookrightarrow L_{p}\left(\Omega_{+}, \mathbb{R}^{n}\right)\right)$. Consequently, the Stokes double layer potential operator $\mathbf{W}: H_{p}^{1}\left(\partial \Omega, \mathbb{R}^{n}\right) \rightarrow B_{p, p^{*}}^{1+\frac{1}{p}}\left(\Omega_{+}, \mathbb{R}^{n}\right)$ is continuous, as asserted. This mapping property combined with the continuity of operator (3.108) implies the continuity of the first operator in (3.68).

Continuity of the second operator in (3.68) follows from similar arguments. To this end, let us mention the useful formula $\mathcal{Q}^{d} \mathbf{g}=\operatorname{div}\left(W_{\triangle} \mathbf{g}\right)$, where the harmonic double layer potential operator $W_{\triangle}: H_{p}^{1}(\partial \Omega) \rightarrow B_{p, p^{*}}^{1+\frac{1}{p}}\left(\Omega_{+}\right)$is continuous (cf., e.g., [59, Proposition 4.23, (2.120), (4.96)]). Thus, the continuity of the Stokes double layer pressure potential operator $\mathcal{Q}^{d}: H_{p}^{1}\left(\partial \Omega, \mathbb{R}^{n}\right) \rightarrow B_{p, p^{*}}^{\frac{1}{p}}\left(\Omega_{+}\right)$immediately follows. This property and continuity of the complementary double layer potential operator $\mathcal{Q}_{\alpha ; 0}^{d}:=\mathcal{Q}_{\alpha}^{d}-\mathcal{Q}^{d}: H_{p}^{1}\left(\partial \Omega, \mathbb{R}^{n}\right) \rightarrow B_{p, p^{*}}^{\frac{1}{p}}\left(\Omega_{+}\right)$, where (cf. [69, (3.10)])

$$
\mathcal{Q}_{\alpha ; 0}^{d} \mathbf{h}=\alpha V_{\triangle}(\mathbf{h} \cdot \boldsymbol{\nu})
$$


yield the continuity of the Brinkman double layer pressure potential operator $\mathcal{Q}_{\alpha}^{d}=\mathcal{Q}^{d}+\mathcal{Q}_{\alpha ; 0}^{d}: H_{p}^{1}\left(\partial \Omega, \mathbb{R}^{n}\right) \rightarrow B_{p, p^{*}}^{\frac{1}{p}}\left(\Omega_{+}\right)$.

Continuity of the first operator in (3.70) for the case $\alpha=0$ is an immediate consequence of [58, Proposition 2.68] applied to the integral operator whose kernel is given by the fundamental stress tensor $\mathbf{S}^{0}$. Moreover, by using again formulas (3.106) and (3.107) we can see that the operator $\mathbf{W}_{\alpha ; 0}: L_{p}\left(\partial \Omega, \mathbb{R}^{n}\right) \rightarrow B_{p, p^{*}}^{\frac{1}{p}}\left(\Omega_{+}, \mathbb{R}^{n}\right)$ is continuous. Therefore, for $\alpha>0$ the first operator in (3.70) is continuous as well. To prove continuity of the second operator in (3.70), we again use the representation $\mathcal{Q}^{d} \mathbf{g}=\operatorname{div}\left(W_{\triangle} \mathbf{g}\right)$, and continuity of the harmonic double layer potential operator $W_{\triangle}: L_{p}(\partial \Omega) \rightarrow B_{p, p^{*}}^{\frac{1}{p}}\left(\Omega_{+}\right)$, e.g., again by $[58$, Proposition 2.68], along with continuity of the complementary double layer potential operator $\mathcal{Q}_{\alpha ; 0}^{d}: L_{p}\left(\partial \Omega, \mathbb{R}^{n}\right) \rightarrow B_{p, p^{*}}^{\frac{1}{p}-1}\left(\Omega_{+}\right)$. Mapping properties (3.65), (3.67) and (3.69) are implied by the ones just above them and by the first relations in (3.35)-(3.36).

(ii) Now, relation (3.94), continuity of the operator $\gamma^{\prime}: B_{p, q}^{s-1}\left(\partial \Omega, \mathbb{R}^{n}\right) \rightarrow B_{p, q}^{s-2+\frac{1}{p}}\left(\mathbb{R}^{n}, \mathbb{R}^{n}\right)$ (cf. Lemma 2.4), and continuity of the Newtonian potential operator $\mathbf{N}_{\alpha ; \mathbb{R}^{n}}: B_{p, q}^{s-2+\frac{1}{p}}\left(\mathbb{R}^{n}, \mathbb{R}^{n}\right) \rightarrow B_{p, q}^{s+\frac{1}{p}}\left(\mathbb{R}^{n}, \mathbb{R}^{n}\right)$ (see (3.11)) imply the continuity of the first operator in (3.72) and thus of the first operators in (3.73) and (3.77). Continuity of the second operator in (3.72) follows by similar arguments based on the equalities $\mathcal{Q}^{s}=\mathcal{Q}_{\mathbb{R}^{n}} \circ \gamma^{\prime}$, and implies also continuity of the second operators in (3.73) and (3.77) (cf. [61, Proposition 10.5.1]).

Further, let us mention that relations (3.106) and (3.107) imply that the operator $\mathbf{W}_{\alpha ; 0}: B_{p, q}^{s}\left(\partial \Omega, \mathbb{R}^{n}\right) \rightarrow B_{p, q}^{s+\frac{1}{p}}\left(\Omega_{+}, \mathbb{R}^{n}\right)$ is continuous for all $p \in(1,+\infty)$ and $s \in(0,1)$. This mapping property combined with the continuity of the Stokes doublelayer potential operator $\left.\mathbf{W}\right|_{\Omega_{+}}: B_{p, q}^{s}\left(\partial \Omega_{+}, \mathbb{R}^{n}\right) \rightarrow B_{p, q}^{s+\frac{1}{p}}\left(\Omega_{+}, \mathbb{R}^{n}\right)$ (see [61, Proposition 10.5.1]) implies the continuity of the first operator in (3.75). The continuity of the second operator in (3.75) can be similarly obtained. Other mapping properties of layer potentials mentioned in (3.72) and (3.79), follow with similar arguments to those for (3.64) and (3.68). We omit the details for the sake of brevity (see also the proof of [32, Lemma 3.4]).

(iii) Equality (3.81) for $\mathbf{g} \in L_{p}\left(\partial \Omega, \mathbb{R}^{n}\right)$ can be obtained by using inequality (3.62) and [47. Proposition 1] (see also [69, Theorems 3.4]). Since $\left(\mathbf{V}_{\alpha} \mathbf{g}\right)_{\text {nt }}^{+}$and $\left(\mathbf{V}_{\alpha} \mathbf{g}\right)_{\text {nt }}^{-}$are well defined for $\mathbf{g} \in H_{p}^{-1}\left(\partial \Omega, \mathbb{R}^{n}\right)$ due to Lemma 3.4(iii), inequality (3.43) and the density argument then imply equality (3.81) also for $\mathbf{g} \in H_{p}^{-1}\left(\partial \Omega, \mathbb{R}^{n}\right)$. Formulas (3.82) and (3.83) follow by using arguments similar to those for the trace formulas (3.11) and (3.18) in [69]. To this end, we first prove the formulas

$$
\left.\left(\partial_{j}\left(V_{i k}^{\alpha} g\right)\right)\right|_{\mathrm{nt}} ^{ \pm}(\mathbf{x})= \pm \frac{1}{2} \nu_{j}(\mathbf{x})\left(\delta_{i k}-\nu_{i}(\mathbf{x}) \nu_{k}(\mathbf{x})\right) g(\mathbf{x})+\text { p.v. } \int_{\partial \Omega} \partial_{j} \mathcal{G}_{i k}^{\alpha}(\mathbf{x}-\mathbf{y}) g(\mathbf{y}) d \sigma_{\mathrm{y}} \quad \text { a.a. } \mathbf{x} \in \partial \Omega
$$

for any $g \in L_{p}(\partial \Omega)$ and all $i, k=1, \ldots, n$, where the function $V_{i k}^{\alpha} g$ is defined as in (3.111) with $\mathcal{G}_{j k}^{\alpha}$ instead of $\mathcal{G}_{j k}$. Indeed, formula (3.116) has been proved in [61, (4.50)] in the case $\alpha=0$. Moreover, the estimate [69, (2.27)] of the kernel $\nabla_{\times} \mathcal{G}_{j k}^{\alpha}(\mathbf{x})-\nabla_{\times} \mathcal{G}_{j k}(\mathbf{x})$ and [47, Proposition 1] imply that there exist the non-tangential limits of the complementary potential $\partial_{j} V_{i k}^{\alpha} g-\partial_{j} V_{i k} g$ at almost all points of $\partial \Omega$, and

$$
\left.\left(\partial_{j}\left(V_{i k}^{\alpha} g\right)-\partial_{j}\left(V_{i k} g\right)\right)\right|_{n t} ^{ \pm}(\mathbf{x})=\text { p.v. } \int_{\partial \Omega}\left(\partial_{j} \mathcal{G}_{i k}^{\alpha}-\partial_{j} \mathcal{G}_{i k}\right)(\mathbf{x}-\mathbf{y}) g(\mathbf{y}) d \sigma_{\mathrm{y}} \quad \text { a.a. } \quad \mathbf{x} \in \partial \Omega,
$$

which implies (3.116) also for $\alpha \neq 0$. Moreover, formula (3.116) yields for any $\mathbf{f} \in L_{p}\left(\partial \Omega, \mathbb{R}^{n}\right)$ that

$$
\left.\left(\partial_{j}\left(\mathbf{V}_{\alpha} \mathbf{f}\right)\right)\right|_{\mathrm{nt}} ^{ \pm}(\mathbf{x})= \pm \frac{1}{2} \nu_{j}(\mathbf{x})\left\{\mathbf{f}(\mathbf{x})-f_{k}(\mathbf{x}) \nu_{k}(\mathbf{x}) \boldsymbol{\nu}(\mathbf{x})\right\}+\text { p.v. } \int_{\partial \Omega} \partial_{j} \mathcal{G}^{\alpha}(\mathbf{x}-\mathbf{y}) \mathbf{f}(\mathbf{y}) d \sigma_{\mathrm{y}} \text { a.a. } \mathbf{x} \in \partial \Omega
$$

(cf. [61, (4.54)] for $\alpha=0$ and [69, Lemma 3.3] for $\alpha>0$ ).

In addition,

$$
\left.\left(\mathcal{Q}^{s} \mathbf{f}\right)\right|_{\mathrm{nt}} ^{ \pm}(\mathbf{x})=\mp \frac{1}{2} \nu_{k}(\mathbf{x}) f_{k}(\mathbf{x})+\text { p.v. } \int_{\partial \Omega} \Pi_{k}(\mathbf{x}-\mathbf{y}) f_{k}(\mathbf{y}) d \sigma_{\mathrm{y}} \quad \text { a.a. } \quad \mathbf{x} \in \partial \Omega
$$

(cf. [61, (4.42)], [69, Lemma 3.3]). Then formulas (3.82) and (3.83) follow from formulas (2.22), (2.24), (3.5), (3.34), (3.118) and (3.119).

Formula (3.84) follows from formula (3.61) and (3.115) together with [61. Proposition 4.2.9] (i.e., the counterpart of the trace formula (3.84) corresponding to the case $\alpha=0$ ).

Continuity of operators (3.74), (3.76), (3.78), (3.80) is implied by the continuity of the operators just above them and by the first relations in (3.35) and (3.36).

Now, we note that formula $\mathcal{V}_{\alpha}=\mathcal{V}+\mathcal{V}_{\alpha ; 0}$, continuity of the Stokes single layer operator $\mathcal{V}: L_{p}\left(\partial \Omega, \mathbb{R}^{n}\right) \rightarrow H_{p}^{1}\left(\partial \Omega, \mathbb{R}^{n}\right)(\mathrm{cf}$. [61, Proposition 4.2.5]), and continuity of the complementary operator $\mathcal{V}_{\alpha ; 0}: L_{p}\left(\partial \Omega, \mathbb{R}^{n}\right) \rightarrow H_{p}^{1}\left(\partial \Omega\right.$, $\left.\mathbb{R}^{n}\right)$ (cf. [33, Theorem 3.4(b)]) imply continuity of the first operator in (3.85). Continuity of the second operator in (3.85) and of the operators in (3.87) similarly follows from [61, Propositions 4.2.7 - 4.2.10] and [33, Theorem 3.4(b)]. In addition, formula (3.47) and the first relation in (3.46) yield the following equality

$$
\left(\mathcal{V}_{\alpha} \mathbf{g}\right)_{j}(\mathbf{x})=\int_{\partial \Omega} \mathcal{G}_{j k}^{\alpha}(\mathbf{x}-\mathbf{y}) g_{0 ; k}(\mathbf{y}) d \sigma_{y}-\sum_{k=1}^{n} \sum_{r, \ell=1}^{n} \text { p.v. } \int_{\partial \Omega}\left(\partial_{\tau_{r \ell}}\left(\mathcal{G}_{j k}^{\alpha}(\mathbf{x}-\mathbf{y})\right)\right) g_{r \ell ; k}(\mathbf{y}) d \sigma_{y} \text { a.a } \mathbf{x} \in \partial \Omega,
$$


for any $\mathbf{g} \in H_{p}^{-1}\left(\partial \Omega, \mathbb{R}^{n}\right.$ ) (cf., e.g., [61, (4.69)] for $\alpha=0$ ). Then the continuity of the first operator in (3.86) immediately follows (see also [61, Proposition 4.2 .5 (iii)] for $\alpha=0$ ). Continuity of the Stokes double layer operator $\mathbf{K}: L_{p}\left(\partial \Omega, \mathbb{R}^{n}\right) \rightarrow L_{p}\left(\partial \Omega, \mathbb{R}^{n}\right)$ (cf., e.g., [61, Corllary 4.2.4]) and the continuity of the reminder operator $\mathbf{K}_{\alpha}-\mathbf{K}: L_{p}\left(\partial \Omega, \mathbb{R}^{n}\right) \rightarrow L_{p}\left(\partial \Omega\right.$, $\left.\mathbb{R}^{n}\right)$ (see [33, Theorem 3.4 (b)]) show the continuity of the second operator in (3.86). Continuity of the traces and conormal derivatives of the layer potentials involved in (3.88)-(3.91) and hence continuity of the boundary operators (3.92), (3.93) immediately follow from the mapping properties of the layer potentials in item (ii) and Lemmas 2.4, 2.11.

Finally, the jump relations given by the first equalities in (3.88)-(3.91) follow from formulas (3.81)-(3.84), together with the density of the embeddings $H_{p}^{1}\left(\partial \Omega, \mathbb{R}^{n}\right) \hookrightarrow B_{p, q}^{s}\left(\partial \Omega, \mathbb{R}^{n}\right)$ and $L_{p}\left(\partial \Omega, \mathbb{R}^{n}\right) \hookrightarrow B_{p, q}^{s-1}\left(\partial \Omega, \mathbb{R}^{n}\right)$, and equivalence results in Theorems 2.5(i) and 2.13(i) for traces and conormal derivatives.

Let us mention the following useful result.

Lemma 3.6 Let $\Omega_{+} \subset \mathbb{R}^{n}(n \geq 3)$ be a bounded Lipschitz domain with connected boundary $\partial \Omega$ and let $\Omega_{-}:=\mathbb{R}^{n} \backslash \bar{\Omega}_{+}$.

(i) If $p \in(1, \infty), \alpha \in(0, \infty)$, $\mathbf{g} \in L_{p}\left(\partial \Omega, \mathbb{R}^{n}\right)$ and $\mathbf{h} \in H_{p}^{1}\left(\partial \Omega, \mathbb{R}^{n}\right)$, then

$$
\begin{aligned}
& \gamma_{ \pm}\left(\mathbf{V}_{\alpha} \mathbf{g}\right)=\left(\mathbf{V}_{\alpha} \mathbf{g}\right)_{\mathrm{nt}}^{ \pm} \in H_{p ; \nu}^{1}\left(\partial \Omega, \mathbb{R}^{n}\right), \\
& \gamma_{ \pm}\left(\mathbf{W}_{\alpha} \mathbf{h}\right)=\left(\mathbf{W}_{\alpha} \mathbf{h}\right)_{\mathrm{nt}}^{ \pm} \in H_{p ; \nu}^{1}\left(\partial \Omega, \mathbb{R}^{n}\right), \\
& \mathbf{t}_{\alpha}^{ \pm}\left(\mathbf{V}_{\alpha} \mathbf{g}, \mathcal{Q}^{s} \mathbf{g}\right)=\mathbf{t}_{\mathrm{nt}}^{ \pm}\left(\mathbf{V}_{\alpha} \mathbf{g}, \mathcal{Q}^{s} \mathbf{g}\right) \in L_{p}\left(\partial \Omega, \mathbb{R}^{n}\right), \\
& \mathbf{t}_{\alpha}^{ \pm}\left(\mathbf{W}_{\alpha} \mathbf{h}, \mathcal{Q}_{\alpha}^{d} \mathbf{h}\right)=\mathbf{t}_{\mathrm{nt}}^{ \pm}\left(\mathbf{W}_{\alpha} \mathbf{h}, \mathcal{Q}_{\alpha}^{d} \mathbf{h}\right) \in L_{p}\left(\partial \Omega, \mathbb{R}^{n}\right)
\end{aligned}
$$

with the corresponding norm estimates

(ii) If $p, q \in(1, \infty), s \in(0,1), \alpha \in(0, \infty), \mathbf{g} \in B_{p, q}^{s-1}\left(\partial \Omega, \mathbb{R}^{n}\right)$ and $\mathbf{h} \in B_{p, q}^{s}\left(\partial \Omega, \mathbb{R}^{n}\right)$, then

$$
\begin{aligned}
& \gamma_{ \pm}\left(\mathbf{V}_{\alpha} \mathbf{g}\right)=\left(\mathbf{V}_{\alpha} \mathbf{g}\right)_{\mathrm{nt}}^{ \pm} \in B_{p, q ; \boldsymbol{\nu}}^{s}\left(\partial \Omega, \mathbb{R}^{n}\right) \\
& \gamma_{ \pm}\left(\mathbf{W}_{\alpha} \mathbf{h}\right)=\left(\mathbf{W}_{\alpha} \mathbf{h}\right)_{\mathrm{nt}}^{ \pm} \in B_{p, q ; \boldsymbol{\nu}}^{s}\left(\partial \Omega, \mathbb{R}^{n}\right)
\end{aligned}
$$

with the corresponding norm estimates.

Proof. Let first $\mathbf{g} \in L_{p}\left(\partial \Omega, \mathbb{R}^{n}\right)$ and $\mathbf{h} \in H_{p}^{1}\left(\partial \Omega, \mathbb{R}^{n}\right), p \in(1, \infty)$. Then, according to Lemma 3.4(ii,v), the right hand sides of the equalities in (3.121)-(3.124) exist almost everywhere on $\partial \Omega$ in the sense of non-tangential limit, while Theorem 3.5(i) yields that $\left(\mathbf{V}_{\alpha} \mathbf{g}, \mathcal{Q}^{s} \mathbf{g}\right),\left(\mathbf{W}_{\alpha} \mathbf{h}, \mathcal{Q}_{\alpha}^{d} \mathbf{h}\right) \in \mathfrak{B}_{p, p^{*} ; \text { div }}^{1+\frac{1}{p}, t}\left(\Omega_{+}, \mathcal{L}_{\alpha}\right)$ and $\left(\mathbf{V}_{\alpha} \mathbf{g}, \mathcal{Q}^{s} \mathbf{g}\right),\left(\mathbf{W}_{\alpha} \mathbf{h}, \mathcal{Q}_{\alpha}^{d} \mathbf{h}\right) \in \mathfrak{B}_{p, p^{*} ; \operatorname{div}, \text { loc }}^{1+\frac{1}{1}, t}\left(\Omega_{-}, \mathcal{L}_{\alpha}\right)$ for any $t \geq-\frac{1}{p^{\prime}}$. Moreover Theorem 3.5 (iii) and the divergence theorem applied to the single layer potentials $\mathbf{V}_{\alpha} \mathbf{g}$ and $\mathbf{W}_{\alpha} \mathbf{h}$ in the domain $\Omega_{+}$yield that $\left(\mathbf{V}_{\alpha} \mathbf{g}\right)_{\mathrm{nt}}^{ \pm} \in H_{p ; \boldsymbol{\nu}}^{1}\left(\partial \Omega, \mathbb{R}^{n}\right), \mathbf{t}_{\mathrm{nt}}^{ \pm}\left(\mathbf{V}_{\alpha} \mathbf{g}, \mathcal{Q}^{s} \mathbf{g}\right) \in L_{p}\left(\partial \Omega, \mathbb{R}^{n}\right)$, for any $\mathbf{g} \in L_{p}\left(\partial \Omega, \mathbb{R}^{n}\right)$, while $\left(\mathbf{W}_{\alpha} \mathbf{h}\right)_{\mathrm{nt}}^{ \pm} \in H_{p ; \boldsymbol{\nu}}^{1}\left(\partial \Omega, \mathbb{R}^{n}\right), \mathbf{t}_{\mathrm{nt}}^{ \pm}\left(\mathbf{W}_{\alpha} \mathbf{g}, \mathcal{Q}^{d} \mathbf{g}\right) \in$ $L_{p}\left(\partial \Omega, \mathbb{R}^{n}\right)$, for any $\mathbf{h} \in H_{p}^{1}\left(\partial \Omega, \mathbb{R}^{n}\right)$, with the corresponding norm estimates. Hence Theorems $2.5(\mathrm{i})$ and $2.13(\mathrm{ii})$ along with Remark 2.14 imply relations (3.121)-(3.124).

For $p, q \in(1, \infty)$ and $s \in(0,1)$, we have $\mathbf{g} \in B_{p, q}^{s-1}\left(\partial \Omega, \mathbb{R}^{n}\right) \subset H_{p}^{-1}\left(\partial \Omega, \mathbb{R}^{n}\right), \mathbf{h} \in B_{p, q}^{s}\left(\partial \Omega, \mathbb{R}^{n}\right) \subset L_{p}\left(\partial \Omega, \mathbb{R}^{n}\right)$ and, according to Lemma 3.4(iii, iv), the right hand sides of the equalities in (3.125) and (3.126) exist almost everywhere on $\partial \Omega$, while Theorem 3.5(ii) yields that $\mathbf{V}_{\alpha} \mathbf{g}, \mathbf{W}_{\alpha} \mathbf{h} \in B_{p, q ; \text { div }}^{s+\frac{1}{p}}\left(\Omega_{+}\right)$. Hence Theorem 2.5(i) implies relations (3.125) and (3.126).

We will further need the following integral representation (the third Green identity) for the homogeneous Brinkman system solution.

Lemma 3.7 Let $\Omega_{+} \subset \mathbb{R}^{n}(n \geq 3)$ be a bounded Lipschitz domain with connected boundary $\partial \Omega$ and let $\Omega_{-}:=\mathbb{R}^{n} \backslash \bar{\Omega}_{+}$. Let $\alpha \in(0, \infty), p, q \in(1, \infty)$ and $s \in(0,1)$. If the the pair $(\mathbf{u}, \pi)$ satisfies the system

$$
\triangle \mathbf{u}-\alpha \mathbf{u}-\nabla \pi=\mathbf{0}, \quad \operatorname{div} \mathbf{u}=0 \text { in } \Omega_{+}
$$

$$
\begin{array}{r}
\text { and }(\mathbf{u}, \pi) \in H_{p}^{s+\frac{1}{p}}\left(\Omega_{+}, \mathbb{R}^{n}\right) \times H_{p}^{s-1-\frac{1}{p}}\left(\Omega_{+}\right) \text {, or }(\mathbf{u}, \pi) \in B_{p, q}^{s+\frac{1}{p}}\left(\Omega_{+}, \mathbb{R}^{n}\right) \times B_{p, q}^{s-1-\frac{1}{p}}\left(\Omega_{+}\right) \text {, then } \\
\mathbf{u}(\mathbf{x})=\mathbf{V}_{\alpha}\left(\mathbf{t}_{\alpha}^{+}(\mathbf{u}, \pi)\right)(\mathbf{x})-\mathbf{W}_{\alpha}\left(\gamma_{+} \mathbf{u}\right)(\mathbf{x}), \forall \mathbf{x} \in \Omega_{+} .
\end{array}
$$

Proof. Let $B(\boldsymbol{y}, \epsilon) \subset \Omega$ be a ball of a radius $\epsilon$ around a point $\boldsymbol{y} \in \Omega_{+}$and let $\mathbf{G}_{k}^{\alpha}(\mathbf{x})=\left(\mathcal{G}_{k 1}^{\alpha}(\mathbf{x}), \ldots, \mathcal{G}_{k n}^{\alpha}(\mathbf{x})\right), k=1, \ldots, n$, where $\left(\mathcal{G}^{\alpha}, \Pi\right)$ is the fundamental solution of the Brinkman system in $\mathbb{R}^{n}$ (see (3.1) and (3.2)). Applying the second Green identity (2.29) in the domain $\Omega_{+} \backslash B(\boldsymbol{y}, \epsilon)$ to $(\mathbf{u}, \pi)$ and to the fundamental solution $\left(\mathbf{G}_{k}^{\alpha}(\cdot-\boldsymbol{y}), \Pi_{k}\right)(\cdot-\boldsymbol{y})$ and taking the limit as $\epsilon \rightarrow 0$, we obtain (3.128).

Next, we show the counterpart of the integral representation formula (3.128) written in terms of the non-tangential trace and conormal derivative.

Lemma 3.8 Let $\Omega_{+} \subset \mathbb{R}^{n}(n \geq 3)$ be a bounded Lipschitz domain with connected boundary $\partial \Omega$. Let $\alpha>0$ and $p \in(1, \infty)$ be given constants. Assume that $M(\mathbf{u}), M(\nabla \mathbf{u}), M(\pi) \in L_{p}(\partial \Omega)$, there exist the non-tangential limits of $\mathbf{u}, \nabla \mathbf{u}$ and $\pi$ at almost all points of the boundary $\partial \Omega$, and that the pair $(\mathbf{u}, \pi)$ satisfies the homogeneous Brinkman system

$$
\Delta \mathbf{u}-\alpha \mathbf{u}-\nabla \pi=\mathbf{0}, \quad \operatorname{div} \mathbf{u}=0 \text { in } \Omega_{+} .
$$


Then u satisfies also the following integral representation formula

$$
\mathbf{u}(\mathbf{x})=\mathbf{V}_{\alpha}\left(\mathbf{t}_{\mathrm{nt}}^{+}(\mathbf{u}, \pi)\right)(\mathbf{x})-\mathbf{W}_{\alpha}\left(\mathbf{u}_{\mathrm{nt}}^{+}\right)(\mathbf{x}), \quad \forall \mathbf{x} \in \Omega_{+} .
$$

Proof. We use arguments similar to the ones in [61, Proposition 4.4.1] for the Stokes system. In the case of a smooth bounded domain $\Omega_{0} \subset \mathbb{R}^{n}$ and for $\mathbf{u} \in C^{2}\left(\bar{\Omega}_{+}, \mathbb{R}^{n}\right), \pi \in C^{1}\left(\bar{\Omega}_{+}\right)$, formula (3.130) follows easily from the integration by parts, cf. e.g. (3.128). Now consider a sequence of sub-domains $\left\{\Omega_{j}\right\}_{j>1}$ in $\Omega_{+}$that contain the point $\mathbf{x} \in \Omega_{+}$and converges to $\Omega_{+}$in the sense of Lemma 2.2. Then formula (3.130) holds for each of the domains $\Omega_{j}$ and by the Lebesgue Dominated Convergence Theorem (applied again after the change of variable as in Lemma 2.2 that reduces the integral over $\partial \Omega_{j}$ to an integral over $\partial \Omega$ ) letting $j \rightarrow \infty$, we obtain (3.130) for the Lipschitz domain $\Omega_{+}$as well.

\section{Invertibility of related integral operators}

Lemma 4.1 Let $\Omega_{+} \subset \mathbb{R}^{n}(n \geq 3)$ be a bounded Lipschitz domain with connected boundary $\partial \Omega$. Let $\alpha \in(0, \infty)$ and $0 \leq s \leq 1$. Then the following operators are isomorphisms,

$$
\begin{aligned}
& \frac{1}{2} \mathbb{I}+\mathbf{K}_{\alpha}^{*}: H_{2}^{-s}\left(\partial \Omega, \mathbb{R}^{n}\right) \rightarrow H_{2}^{-s}\left(\partial \Omega, \mathbb{R}^{n}\right), \\
& \frac{1}{2} \mathbb{I}+\mathbf{K}_{\alpha}: H_{2}^{s}\left(\partial \Omega, \mathbb{R}^{n}\right) \rightarrow H_{2}^{s}\left(\partial \Omega, \mathbb{R}^{n}\right) .
\end{aligned}
$$

Proof. Isomorphism property of operator (4.1) for $s=0$ follows from [46, Proposition 7.1] (see also [69, Lemma 5.1]). By duality this also implies the isomorphism property of operator (4.2) for $s=0$.

Let us now remark that for $\alpha=0$ and $0<s \leq 1$, operator (4.2) is a Fredholm operator with index zero (cf., e.g., [61, Proposition 10.5.3 and Theorem 5.3.6]), while the operator $\mathbf{K}_{\alpha ; 0}:=\mathbf{K}_{\alpha}-\mathbf{K}: H_{2}^{s}\left(\partial \Omega, \mathbb{R}^{n}\right) \rightarrow H_{2}^{s}\left(\partial \Omega\right.$, $\left.\mathbb{R}^{n}\right)$ is compact (cf., e.g., [33, Theorem 3.4]), implying that for $\alpha>0$ and $0<s \leq 1,(4.2)$ is a Fredholm operator with index zero as well. Then by Lemma B.4 and the invertibility property of operator (4.2) for $s=0$ we obtain the equalities

$$
\operatorname{Ker}\left\{\frac{1}{2} \mathbb{I}+\mathbf{K}_{\alpha}: H_{2}^{s}\left(\partial \Omega, \mathbb{R}^{n}\right) \rightarrow H_{2}^{s}\left(\partial \Omega, \mathbb{R}^{n}\right)\right\}=\operatorname{Ker}\left\{\frac{1}{2} \mathbb{I}+\mathbf{K}_{\alpha}: H_{2}^{0}\left(\partial \Omega, \mathbb{R}^{n}\right) \rightarrow H_{2}^{0}\left(\partial \Omega, \mathbb{R}^{n}\right)\right\}=\{\mathbf{0}\}, \quad 0<s \leq 1,
$$

which show invertibility and hence isomorphism property of operator (4.2) for $\alpha>0$ and $0<s \leq 1$ as well. A duality argument implies that operator (4.1) is also an isomorphism whenever $\alpha>0$ and $0<s \leq 1$.

We will often need the following two intervals,

$$
\mathcal{R}_{0}(n, \varepsilon)=\left(\frac{2(n-1)}{n+1}-\varepsilon, 2+\varepsilon\right) \cap(1,+\infty), \quad \mathcal{R}_{1}(n, \varepsilon)= \begin{cases}(2-\varepsilon,+\infty) & \text { if } n=3 \\ \left(2-\varepsilon, \frac{2(n-1)}{n-3}+\varepsilon\right) & \text { if } n>3\end{cases}
$$

which are particular cases of a more general interval

$$
\mathcal{R}_{\theta}(n, \varepsilon)= \begin{cases}(2-\varepsilon,+\infty) & \text { if } n=3 \text { and } \theta=1 \\ \left(\frac{2(n-1)}{n+1-2 \theta}-\varepsilon, \frac{2(n-1)}{n-1-2 \theta}+\varepsilon\right) \cap(1,+\infty) & \text { if } n>3 \text { or } 0 \leq \theta<1\end{cases}
$$

Lemma 4.2 Let $\Omega_{+} \subset \mathbb{R}^{n}(n \geq 3)$ be a bounded Lipschitz domain with connected boundary $\partial \Omega$. Let $\alpha \in(0, \infty)$. Then there exists $\varepsilon=\varepsilon(\partial \Omega)>0$ such that for any $p \in \mathcal{R}_{0}(n, \varepsilon)$ and $p^{\prime} \in \mathcal{R}_{1}(n, \varepsilon)$, see (4.4), the following operators are isomorphisms,

$$
\begin{aligned}
& \frac{1}{2} \mathbb{I}+\mathbf{K}_{\alpha}^{*}: L_{p}\left(\partial \Omega, \mathbb{R}^{n}\right) \rightarrow L_{p}\left(\partial \Omega, \mathbb{R}^{n}\right), \\
& \frac{1}{2} \mathbb{I}+\mathbf{K}_{\alpha}^{*}: H_{p^{\prime}}^{-1}\left(\partial \Omega, \mathbb{R}^{n}\right) \rightarrow H_{p^{\prime}}^{-1}\left(\partial \Omega, \mathbb{R}^{n}\right), \\
& \frac{1}{2} \mathbb{I}+\mathbf{K}_{\alpha}: L_{p^{\prime}}\left(\partial \Omega, \mathbb{R}^{n}\right) \rightarrow L_{p^{\prime}}\left(\partial \Omega, \mathbb{R}^{n}\right), \\
& \frac{1}{2} \mathbb{I}+\mathbf{K}_{\alpha}: H_{p}^{1}\left(\partial \Omega, \mathbb{R}^{n}\right) \rightarrow H_{p}^{1}\left(\partial \Omega, \mathbb{R}^{n}\right) .
\end{aligned}
$$

If $\Omega_{+}$is of class $C^{1}$, then the above invertibility properties hold for all $p, p^{\prime} \in(1, \infty)$.

Proof. By [61, Theorem 9.1.11] there exists a parameter $\varepsilon>0$ such that for any $p \in \mathcal{R}_{0}(n, \varepsilon)$,

$$
\frac{1}{2} \mathbb{I}+\mathbf{K}^{*}: L_{p}\left(\partial \Omega, \mathbb{R}^{n}\right) \rightarrow L_{p}\left(\partial \Omega, \mathbb{R}^{n}\right)
$$


is a Fredholm operator with index zero. Then compactness of the operator $\mathbf{K}_{\alpha ; 0}^{*}:=\mathbf{K}_{\alpha}^{*}-\mathbf{K}^{*}: L_{p}\left(\partial \Omega, \mathbb{R}^{n}\right) \rightarrow L_{p}\left(\partial \Omega, \mathbb{R}^{n}\right)$ for any $p \in(1, \infty)$ (see [33, Theorem 3.4(b)]), imply that operator (4.6) is Fredholm with index zero as well, for any $p \in \mathcal{R}_{0}(n, \varepsilon)$. In addition, a density argument based on Lemma B.4 and the invertibility property of operator (4.1) in the case $s=0$, show that operator (4.6) is an isomorphism for $p=2$ and hence for any $p \in \mathcal{R}_{0}(n, \varepsilon)$.

Similarly, by [61, Theorem 9.1.3] there exists a parameter (for the sake of brevity, we use the same notation as above) $\varepsilon>0$ such that for any $p \in \mathcal{R}_{0}(n, \varepsilon)$ the operator

$$
\frac{1}{2} \mathbb{I}+\mathbf{K}: H_{p}^{1}\left(\partial \Omega, \mathbb{R}^{n}\right) \rightarrow H_{p}^{1}\left(\partial \Omega, \mathbb{R}^{n}\right)
$$

is Fredholm with index zero. Then compactness of the complementary operator $\mathbf{K}_{\alpha ; 0}:=\mathbf{K}_{\alpha}-\mathbf{K}: H_{p}^{1}\left(\partial \Omega, \mathbb{R}^{n}\right) \rightarrow H_{p}^{1}\left(\partial \Omega, \mathbb{R}^{n}\right)$ for any $p \in(1, \infty)$ (see [33, Theorem 3.4(b)]), implies that operator (4.9) is Fredholm with index zero as well, for any $p \in \mathcal{R}_{0}(n, \varepsilon)$. In addition, a density argument based on Lemma B.4 and the invertibility property for operator (4.2) in the case $s=1$, show that operator (4.9) is an isomorphism for $p=2$ and hence for any $p \in \mathcal{R}_{0}(n, \varepsilon)$.

Isomorphism property of operators (4.7) and (4.8) then follow by duality and isomorphism property of operators (4.9) and (4.6), respectively, for $p^{\prime}=\frac{p}{p-1}$.

If $\Omega_{+}$is of class $C^{1}$, then operator (4.11) is Fredholm with index zero for any $p \in(1, \infty)$, cf., e.g., [67, Remark 3.1], and the the rest of the proof holds true for any $p, q \in(1, \infty)$.

Lemmas 4.2, A.1 and B.1 (ii) and an interpolation argument (provided by the complex and real interpolation theory) imply the following assertion.

Corollary 4.3 Let $\Omega_{+} \subset \mathbb{R}^{n}(n \geq 3)$ be a bounded Lipschitz domain with connected boundary $\partial \Omega$, and $\alpha \in(0, \infty)$. Then there exists $\varepsilon=\varepsilon(\partial \Omega)>0$ such that for any $p \in \mathcal{R}_{s}(n, \varepsilon)$ and $p^{\prime} \in \mathcal{R}_{1-s}(n, \varepsilon)$, cf. (4.5), the following operators are isomorphisms

$$
\begin{aligned}
& \frac{1}{2} \mathbb{I}+\mathbf{K}_{\alpha}: H_{p^{\prime}}^{s}\left(\partial \Omega, \mathbb{R}^{n}\right) \rightarrow H_{p^{\prime}}^{s}\left(\partial \Omega, \mathbb{R}^{n}\right), \quad s \in[0,1], \\
& \frac{1}{2} \mathbb{I}+\mathbf{K}_{\alpha}^{*}: H_{p}^{-s}\left(\partial \Omega, \mathbb{R}^{n}\right) \rightarrow H_{p}^{-s}\left(\partial \Omega, \mathbb{R}^{n}\right), \quad s \in[0,1], \\
& \frac{1}{2} \mathbb{I}+\mathbf{K}_{\alpha}: B_{p^{\prime}, q}^{s}\left(\partial \Omega, \mathbb{R}^{n}\right) \rightarrow B_{p^{\prime}, q}^{s}\left(\partial \Omega, \mathbb{R}^{n}\right), \quad s \in(0,1), q \in(1, \infty), \\
& \frac{1}{2} \mathbb{I}+\mathbf{K}_{\alpha}^{*}: B_{p, q}^{-s}\left(\partial \Omega, \mathbb{R}^{n}\right) \rightarrow B_{p, q}^{-s}\left(\partial \Omega, \mathbb{R}^{n}\right), \quad s \in(0,1), q \in(1, \infty) .
\end{aligned}
$$

If $\Omega_{+}$is of class $C^{1}$, then the properties hold for all $p, p^{\prime} \in(1, \infty)$.

Next we show the following invertibility result (see also [46. Proposition 7.2] in the case $p=2$ and $s=0$ ).

Lemma 4.4 Let $\Omega_{+} \subset \mathbb{R}^{n}(n \geq 3)$ be a bounded Lipschitz domain with connected boundary $\partial \Omega$ and let $\Omega_{-}:=\mathbb{R}^{n} \backslash \bar{\Omega}_{+}$. Let $\alpha \in(0, \infty)$. Then there exists a number $\varepsilon=\varepsilon(\partial \Omega)>0$ such that the operators

$$
\begin{aligned}
& -\frac{1}{2} \mathbb{I}+\mathbf{K}_{\alpha}: L_{p^{\prime} ; \boldsymbol{\nu}}\left(\partial \Omega, \mathbb{R}^{n}\right) \rightarrow L_{p^{\prime} ; \boldsymbol{\nu}}\left(\partial \Omega, \mathbb{R}^{n}\right), \\
& -\frac{1}{2} \mathbb{I}+\mathbf{K}_{\alpha}^{*}: L_{p}\left(\partial \Omega, \mathbb{R}^{n}\right) / \mathbb{R} \boldsymbol{\nu} \rightarrow L_{p}\left(\partial \Omega, \mathbb{R}^{n}\right) / \mathbb{R} \boldsymbol{\nu}, \\
& -\frac{1}{2} \mathbb{I}+\mathbf{K}_{\alpha}: H_{p ; \nu}^{1}\left(\partial \Omega, \mathbb{R}^{n}\right) \rightarrow H_{p ; \nu}^{1}\left(\partial \Omega, \mathbb{R}^{n}\right), \\
& -\frac{1}{2} \mathbb{I}+\mathbf{K}_{\alpha}^{*}: H_{p^{\prime}}^{-1}\left(\partial \Omega, \mathbb{R}^{n}\right) / \mathbb{R} \boldsymbol{\nu} \rightarrow H_{p^{\prime}}^{-1}\left(\partial \Omega, \mathbb{R}^{n}\right) / \mathbb{R} \boldsymbol{\nu}
\end{aligned}
$$

are isomorphisms for all $p \in \mathcal{R}_{0}(n, \varepsilon)$ and $p^{\prime} \in \mathcal{R}_{1}(n, \varepsilon)(c f .(4.4))$.

If the domain $\Omega$ is of class $C^{1}$, the above properties hold for all $p, p^{\prime} \in(1, \infty)$.

Proof. In the case $\alpha=0$, operator (4.16) is an isomorphism (cf. [61, Corollary 9.1.12]), and hence a Fredholm operator with index zero for any $p^{\prime} \in \mathcal{R}_{1}(n, \varepsilon)$. Moreover, the operator $\mathbf{K}_{\alpha}-\mathbf{K}$ is compact on the space $L_{p^{\prime}}\left(\partial \Omega, \mathbb{R}^{n}\right)$ (see [33, Theorem 3.4(b)]), and its range is a subset of $L_{p^{\prime} ; \nu}\left(\partial \Omega, \mathbb{R}^{n}\right)$. Indeed, by using the formula

$$
\left(\mathbf{K}_{\alpha}-\mathbf{K}\right) \mathbf{h}=\left(-\frac{1}{2} \mathbb{I}+\mathbf{K}_{\alpha}\right) \mathbf{h}-\left(-\frac{1}{2} \mathbb{I}+\mathbf{K}\right) \mathbf{h}=\gamma_{+} \mathbf{W}_{\alpha} \mathbf{h}-\gamma_{+} \mathbf{W h}
$$

the equations $\operatorname{div} \mathbf{W}_{\alpha} \mathbf{h}=0$ and $\operatorname{div} \mathbf{W h}=0$ in $\Omega_{+}$, and then, the divergence theorem and the trace formulas (3.82), we deduce that $\left(\mathbf{K}_{\alpha}-\mathbf{K}\right) \mathbf{h} \in L_{p^{\prime} ; \boldsymbol{\nu}}\left(\partial \Omega, \mathbb{R}^{n}\right)$ for any $\mathbf{h} \in L_{p^{\prime} ; \boldsymbol{\nu}}\left(\partial \Omega, \mathbb{R}^{n}\right)$. Therefore, the operator $\mathbf{K}_{\alpha}-\mathbf{K}: L_{p^{\prime} ; \boldsymbol{\nu}}\left(\partial \Omega, \mathbb{R}^{n}\right) \rightarrow L_{p^{\prime} ; \boldsymbol{\nu}}\left(\partial \Omega, \mathbb{R}^{n}\right)$ is compact, and then operator (4.16) is Fredholm with index zero for any $p^{\prime} \in \mathcal{R}_{1}(n, \varepsilon)$. On the other hand, by a similar reasoning (cf., e.g., [61, Theorem 9.1.3] and [33, Theorem 3.4 (b)]), operator (4.18) is Fredholm with index zero as well, for any $p \in \mathcal{R}_{0}(n, \varepsilon)$. 
We show now that operators (4.16) and (4.18) are also injective. Let us start from operator (4.18) with $p=2$. Let $\mathbf{h}_{0} \in H_{2 ; \boldsymbol{\nu}}^{1}\left(\partial \Omega, \mathbb{R}^{n}\right)$ be such that $\left(-\frac{1}{2} \mathbb{I}+\mathbf{K}_{\alpha}\right) \mathbf{h}=\mathbf{0}$. Thus, $\gamma_{+} \mathbf{W}_{\alpha} \mathbf{h}_{0}=\mathbf{0}$, and by applying the Green formula (2.28) to the double layer velocity and pressure potentials $\mathbf{W}_{\alpha} \mathbf{h}_{0}$ and $\mathcal{Q}_{\alpha}^{d} \mathbf{h}_{0}$ in $\Omega_{+}$, we deduce that $\mathbf{W}_{\alpha} \mathbf{h}_{0}=\mathbf{0}$ and $\mathcal{Q}_{\alpha}^{d} \mathbf{h}_{0}=c_{0} \in \mathbb{R}$ in $\Omega_{+}$. According to formula (3.84), we obtain that $\mathbf{t}_{\mathrm{nt}}^{-}\left(\mathbf{W}_{\alpha} \mathbf{h}_{0}, \mathcal{Q}_{\alpha}^{d} \mathbf{h}_{0}\right)=\mathbf{t}_{\mathrm{nt}}^{+}\left(\mathbf{W}_{\alpha} \mathbf{h}_{0}, \mathcal{Q}_{\alpha}^{d} \mathbf{h}_{0}\right)=-c_{0} \boldsymbol{\nu}$, and then the relation $\gamma_{-} \mathbf{W}_{\alpha} \mathbf{h}_{0}=\mathbf{h}_{0} \in H_{2 ; \nu}^{1}\left(\partial \Omega, \mathbb{R}^{n}\right)$ shows that $\left\langle\mathbf{t}_{\mathrm{nt}}^{-}\left(\mathbf{W}_{\alpha} \mathbf{h}_{0}, \mathcal{Q}_{\alpha}^{d} \mathbf{h}_{0}\right), \gamma_{-} \mathbf{W}_{\alpha} \mathbf{h}_{0}\right\rangle_{\partial \Omega}=0$. Finally, the relations $\mathbf{W}_{\alpha} \mathbf{h}_{0}(\mathbf{x})=O\left(|\mathbf{x}|^{-n}\right)$ and $\mathcal{Q}^{d} \mathbf{h}_{0}=O\left(|\mathbf{x}|^{1-n}\right.$ ) as $|\mathbf{x}| \rightarrow \infty$ (see, e.g., [74, Lemma 2.12, (2.76)]), and the Green formula (2.28) applied to $\mathbf{W}_{\alpha} \mathbf{h}_{0}$ and $\mathcal{Q}_{\alpha}^{d} \mathbf{h}_{0}$ in $\Omega_{-}$imply that $\mathbf{W}_{\alpha} \mathbf{h}_{0}=\mathbf{0}$ and $\mathcal{Q}_{\alpha}^{d} \mathbf{h}_{0}=\mathbf{0}$ in $\Omega_{-}$. Then the trace formula (3.82) yields that $\mathbf{h}_{0}=\mathbf{0}$. Consequently, operator (4.18) with $p=2$ is injective. Then Lemma B.4 implies that operator (4.16) with $p^{\prime}=2$ is injective as well. Applying Lemma B.4 again, we now obtain that operator (4.18) with $p \in \mathcal{R}_{0}(n, \varepsilon)$ and operator (4.16) with $p^{\prime} \in \mathcal{R}_{1}(n, \varepsilon)$ are injective, and according to their Fredholm property, these operators are also isomorphisms. Operators (4.17) and (4.19) are then isomorphisms by duality.

If $\Omega$ is of $C^{1}$ class, then for all $p, p^{\prime} \in(1, \infty)$ operators $(4.16)$ and $(4.17)$ are Fredholm with index zero due to compactness of the operators $\mathbf{K}$ and $\mathbf{K}^{*}$ on the corresponding spaces (cf., e.g., [22, Eq. (3.51) in the proof of Proposition 3.5]), and [33, Theorem 3.4 (b)]. Then the previous paragraph implies that operators (4.16)-(4.19) are isomorphisms for $p, p^{\prime} \in(1, \infty)$.

Lemmas 4.4, A.1 and B.1(ii) by interpolation imply the following result (see also [46. Proposition 7.2] for $p=2$ and $s=0$ ).

Corollary 4.5 Let $\Omega_{+} \subset \mathbb{R}^{n}(n \geq 3)$ be a bounded Lipschitz domain with connected boundary $\partial \Omega$ and let $\Omega_{-}:=\mathbb{R}^{n} \backslash \bar{\Omega}_{+}$. Let $\alpha \in(0, \infty)$. Then there exists $\varepsilon=\varepsilon(\partial \Omega)>0$ such that for any $p \in \mathcal{R}_{s}(n, \varepsilon)$ and $p^{\prime} \in \mathcal{R}_{1-s}(n, \varepsilon)($ cf. (4.5)), the following operators are isomorphisms,

$$
\begin{aligned}
& -\frac{1}{2} \mathbb{I}+\mathbf{K}_{\alpha}: H_{p^{\prime} ; \nu}^{s}\left(\partial \Omega, \mathbb{R}^{n}\right) \rightarrow H_{p^{\prime} ; \nu}^{s}\left(\partial \Omega, \mathbb{R}^{n}\right), s \in[0,1], \\
& -\frac{1}{2} \mathbb{I}+\mathbf{K}_{\alpha}^{*}: H_{p}^{-s}\left(\partial \Omega, \mathbb{R}^{n}\right) / \mathbb{R} \boldsymbol{\nu} \rightarrow H_{p}^{-s}\left(\partial \Omega, \mathbb{R}^{n}\right) / \mathbb{R} \boldsymbol{\nu}, \quad s \in[0,1], \\
& -\frac{1}{2} \mathbb{I}+\mathbf{K}_{\alpha}: B_{p^{\prime}, q ; \nu}^{s}\left(\partial \Omega, \mathbb{R}^{n}\right) \rightarrow B_{p^{\prime}, q ; \nu}^{s}\left(\partial \Omega, \mathbb{R}^{n}\right), \quad s \in(0,1), \quad q \in(1, \infty), \\
& -\frac{1}{2} \mathbb{I}+\mathbf{K}_{\alpha}^{*}: B_{p, q}^{-s}\left(\partial \Omega, \mathbb{R}^{n}\right) / \mathbb{R} \boldsymbol{\nu} \rightarrow B_{p, q}^{-s}\left(\partial \Omega, \mathbb{R}^{n}\right) / \mathbb{R} \boldsymbol{\nu}, \quad s \in(0,1), q \in(1, \infty) .
\end{aligned}
$$

If $\Omega_{+}$is of class $C^{1}$, then the properties hold for all $p, p^{\prime} \in(1, \infty)$.

In the case $\alpha=0$, the result, corresponding to the next one, has been obtained in [61, Theorem 9.1.4, Corollary 9.1.5] (see also [62, Theorem 6.1]).

Lemma 4.6 Let $\Omega_{+} \subset \mathbb{R}^{n}(n \geq 3)$ be a bounded Lipschitz domain with connected boundary $\partial \Omega$ and let $\Omega_{-}:=\mathbb{R}^{n} \backslash \bar{\Omega}_{+}$. Let $\alpha \in(0, \infty)$. Then there exists a number $\varepsilon>0$ such that for any $p \in \mathcal{R}_{0}(n, \varepsilon)$ and $p^{\prime} \in \mathcal{R}_{1}(n, \varepsilon)$, see (4.4), the following Brinkman single layer potential operators are isomorphisms

$$
\begin{aligned}
& \mathcal{V}_{\alpha}: L_{p}\left(\partial \Omega, \mathbb{R}^{n}\right) / \mathbb{R} \nu \rightarrow H_{p ; \nu}^{1}\left(\partial \Omega, \mathbb{R}^{n}\right) \\
& \mathcal{V}_{\alpha}: H_{p^{\prime}}^{-1}\left(\partial \Omega, \mathbb{R}^{n}\right) / \mathbb{R} \boldsymbol{\nu} \rightarrow L_{p^{\prime} ; \nu}\left(\partial \Omega, \mathbb{R}^{n}\right) .
\end{aligned}
$$

If $\Omega_{+}$is of class $C^{1}$, then the above invertibility properties hold for all $p, p^{\prime} \in(1, \infty)$.

Proof. First, we note that for any $\mathbf{f} \in L_{p}\left(\partial \Omega, \mathbb{R}^{n}\right)$ the inclusion $\mathcal{V}_{\alpha} \mathbf{f} \in H_{p}^{1}\left(\partial \Omega, \mathbb{R}^{n}\right)$ follows by Theorem 3.5(iii). Moreover, the inclusion $\mathcal{V}_{\alpha} \mathbf{f} \in H_{p ; \nu}^{1}\left(\partial \Omega, \mathbb{R}^{n}\right)$ follows from the equation $\operatorname{div} \mathbf{V}_{\alpha} \mathbf{f}=0$ in $\Omega_{+}$, the divergence theorem and relation (3.88). On the other hand, there exists a number $\varepsilon>0$ such that the Stokes single layer potential operator

$$
\mathcal{V}: L_{p}\left(\partial \Omega, \mathbb{R}^{n}\right) / \mathbb{R} \boldsymbol{\nu} \rightarrow H_{p ; \nu}^{1}\left(\partial \Omega, \mathbb{R}^{n}\right)
$$

is an isomorphism for any $p \in \mathcal{R}_{0}(n, \varepsilon)$ (cf. [61, Theorem 9.1.4]), which implies that $\mathcal{V}: L_{p}\left(\partial \Omega, \mathbb{R}^{n}\right) \rightarrow H_{p}^{1}\left(\partial \Omega, \mathbb{R}^{n}\right)$ is a Fredholm operator with index zero for the same range of $p$. Thus, the Brinkman single layer potential operator

$$
\mathcal{V}_{\alpha}: L_{p}\left(\partial \Omega, \mathbb{R}^{n}\right) \rightarrow H_{p}^{1}\left(\partial \Omega, \mathbb{R}^{n}\right)
$$

is a Fredholm operator of index zero for any $p \in \mathcal{R}_{0}(n, \varepsilon)$, as follows from the equality $\mathcal{V}_{\alpha}=\mathcal{V}+\mathcal{V}_{\alpha ; 0}$, where $\mathcal{V}_{\alpha ; 0}:=\mathcal{V}_{\alpha}-\mathcal{V}$ : $L_{p}\left(\partial \Omega, \mathbb{R}^{n}\right) \rightarrow H_{p}^{1}\left(\partial \Omega, \mathbb{R}^{n}\right)$ is a compact operator (cf. [33, Lemma 3.1]). Then by Lemma B.4, we obtain the equality

$$
\operatorname{Ker}\left\{\mathcal{V}_{\alpha}: L_{p}\left(\partial \Omega, \mathbb{R}^{n}\right) \rightarrow H_{p}^{1}\left(\partial \Omega, \mathbb{R}^{n}\right)\right\}=\operatorname{Ker}\left\{\mathcal{V}_{\alpha}: L_{2}\left(\partial \Omega, \mathbb{R}^{n}\right) \rightarrow H_{2}^{1}\left(\partial \Omega, \mathbb{R}^{n}\right)\right\}
$$

for each $p \in \mathcal{R}_{0}(n, \varepsilon)$.

Moreover, by considering a density $\boldsymbol{\varphi}_{0} \in L_{2}\left(\partial \Omega, \mathbb{R}^{n}\right)$ such that $\mathcal{V}_{\alpha} \boldsymbol{\varphi}_{0}=\mathbf{0}$ on $\partial \Omega$, by applying the Green identity (2.28) to the single layer velocity and pressure potentials $\mathbf{u}_{0}=\mathbf{V}_{\alpha} \boldsymbol{\varphi}_{0}$ and $\pi_{0}=\mathcal{Q}^{s} \boldsymbol{\varphi}_{0}$, and by using Theorem 3.5, we deduce that $\mathbf{u}_{0}=\mathbf{0}$ and $\pi_{0}=c_{0} \in \mathbb{R}$ in $\Omega_{+}$. In addition, the behavior at infinity of the single layer potentials, $\mathbf{u}_{0}(\mathbf{x})=O\left(|\mathbf{x}|^{-n}\right), \boldsymbol{\sigma}\left(\mathbf{u}_{0}, \pi_{0}\right)(\mathbf{x})=O\left(|\mathbf{x}|^{1-n}\right)$ as $|\mathbf{x}| \rightarrow \infty$ (see, e.g., [46, Section 4]), yields that the Green identity (2.28) applies also to the fields $\mathbf{u}_{0}$ and $\pi_{0}$ in the exterior 
domain $\Omega_{-}$and yields $\mathbf{u}_{0}=\mathbf{0}, \pi_{0}=0$ in $\Omega_{-}$. Then by formulas (3.83) $\boldsymbol{\varphi}_{0}=c_{0} \boldsymbol{\nu}$. On the other hand, the divergence theorem and the second equation in (3.4) imply that $\left(\mathbf{V}_{\alpha} \boldsymbol{\nu}\right)_{j}(x)=\int_{\Omega_{+}} \frac{\partial \mathcal{G}_{j k}^{\alpha}(x-y)}{\partial y_{k}} d y=0$, and accordingly that $\mathcal{V}_{\alpha} \boldsymbol{\nu}=\mathbf{0}$. Thus, we obtain the equality

$$
\operatorname{Ker}\left\{\mathcal{V}_{\alpha}: L_{2}\left(\partial \Omega, \mathbb{R}^{n}\right) \rightarrow H_{2}^{1}\left(\partial \Omega, \mathbb{R}^{n}\right)\right\}=\mathbb{R} \boldsymbol{\nu}
$$

Therefore, by (4.27) the codimension of the range of the operator $\mathcal{V}_{\alpha}: L_{p}\left(\partial \Omega, \mathbb{R}^{n}\right) \rightarrow H_{p}^{1}\left(\partial \Omega, \mathbb{R}^{n}\right)$ is equal to one. Moreover, Range $\left(\mathcal{V}_{\alpha ; \partial \Omega}\right) \subseteq H_{p ; \nu}^{1}\left(\partial \Omega, \mathbb{R}^{n}\right)$, as follows from the divergence theorem and the second equation in (3.4). Since $H_{p ; \nu}^{1}\left(\partial \Omega, \mathbb{R}^{n}\right)$ is a subspace of codimension one in $H_{p}^{1}\left(\partial \Omega, \mathbb{R}^{n}\right)$, we conclude that the range of the operator $\mathcal{V}_{\alpha}: L_{p}\left(\partial \Omega, \mathbb{R}^{n}\right) \rightarrow H_{p}^{1}\left(\partial \Omega, \mathbb{R}^{n}\right)$ is just $H_{p, \nu}^{1}\left(\partial \Omega_{j}, \mathbb{R}^{n}\right)$. Then the Fundamental quotient theorem for linear continuous maps implies $\mathcal{V}_{\alpha}: L_{p}\left(\partial \Omega, \mathbb{R}^{n}\right) / \mathbb{R} \boldsymbol{\nu} \rightarrow H_{p ; \nu}^{1}\left(\partial \Omega, \mathbb{R}^{n}\right)$ is an isomorphism for any $p \in \mathcal{R}_{0}(n, \varepsilon)$, as asserted.

Since the operator $\mathcal{V}_{\alpha}$ is self-adjoint, duality shows that operator (4.25) is also an isomorphism for any $q \in(1, \infty)$ such that $q=\frac{p}{p-1}$. Note that for the same range of $q$, the Stokes single layer potential operator $\mathcal{V}: H_{q}^{-1}\left(\partial \Omega, \mathbb{R}^{n}\right) / \mathbb{R} \boldsymbol{\nu} \rightarrow L_{q ; \nu}^{1}\left(\partial \Omega, \mathbb{R}^{n}\right)$ is an isomorphism as well (see [61, Corollary 9.1.5] for $\alpha=0$ ).

If $\Omega_{+}$is of class $C^{1}$, then the operator $\mathcal{V}: H_{q}^{-1}\left(\partial \Omega, \mathbb{R}^{n}\right) \rightarrow L_{p}\left(\partial \Omega, \mathbb{R}^{n}\right)$ is Fredholm with index zero for any $q \in(1, \infty)(\mathrm{cf} .$, e.g., [67, Remark 3.1]; see also [28, Proposition 4.1]). By duality, we deduce that operator (4.26) is Fredholm with index zero as well for any $p \in(1, \infty)$ whenever $\alpha=0$. In view of [33, Theorem 3.4], the complementary operator $\mathcal{V}_{\alpha}-\mathcal{V}: L_{p}\left(\partial \Omega, \mathbb{R}^{n}\right) \rightarrow H_{p}^{1}\left(\partial \Omega, \mathbb{R}^{n}\right)$ is compact (even in the case of a Lipschitz domain). Therefore, the operator $\mathcal{V}_{\alpha}: L_{p}\left(\partial \Omega, \mathbb{R}^{n}\right) \rightarrow H_{p}^{1}\left(\partial \Omega, \mathbb{R}^{n}\right)$ is Fredholm with index zero for any $p \in(1, \infty)$. Then the rest of the proof holds true for any $p, q \in(1, \infty)$.

Lemmas 4.6, A.1 and B.1(ii) and an interpolation argument imply the following assertion (see also [67, Remark 3.1] in the case of a $C^{1}$ domain).

Corollary 4.7 Let $\Omega_{+} \subset \mathbb{R}^{n}(n \geq 3)$ be a bounded Lipschitz domain with connected boundary $\partial \Omega$ and let $\Omega_{-}:=\mathbb{R}^{n} \backslash \bar{\Omega}_{+}$. Let $\alpha \in(0, \infty)$ and $p \in \mathcal{R}_{s}(n, \epsilon)$, see (4.5). Then there exists $\varepsilon=\varepsilon(\partial \Omega)>0$ such that the following operators are isomorphisms,

$$
\begin{aligned}
& \mathcal{V}_{\alpha}: H_{p}^{-s}\left(\partial \Omega, \mathbb{R}^{n}\right) / \mathbb{R} \boldsymbol{\nu} \rightarrow H_{p ; \nu}^{1-s}\left(\partial \Omega, \mathbb{R}^{n}\right), \quad s \in[0,1], \\
& \mathcal{V}_{\alpha}: B_{p, q}^{-s}\left(\partial \Omega, \mathbb{R}^{n}\right) / \mathbb{R} \boldsymbol{\nu} \rightarrow B_{p, q ; \nu}^{1-s}\left(\partial \Omega, \mathbb{R}^{n}\right), \quad s \in(0,1), q \in(1, \infty) .
\end{aligned}
$$

If $\Omega_{+}$is of class $C^{1}$, then the property holds for any $p \in(1, \infty)$.

\section{The Dirichlet and Neumann problems for the Brinkman system}

\subsection{The Dirichlet problem for the Brinkman system}

Let us consider the Dirichlet problem for the homogeneous Brinkman system,

$$
\begin{aligned}
& \triangle \mathbf{u}-\alpha \mathbf{u}-\nabla \pi=\mathbf{0}, \quad \operatorname{div} \mathbf{u}=0 \text { in } \Omega_{+}, \\
& \mathbf{u}_{\mathrm{nt}}^{+}=\mathbf{h}_{0} \text { on } \partial \Omega,
\end{aligned}
$$

and show the following assertion (cf. [69, Theorem 5.5] for $p=2$ and the boundary data in the space $L_{2 ; \nu}\left(\partial \Omega, \mathbb{R}^{n}\right)$; for $\alpha=0$ see also [61, Corollary 9.1.5, Theorems 9.1.4, 9.2.2 and 9.2.5] and [62, Theorem 7.1]). The Dirichlet boundary condition (5.2) is understood in the sense of non-tangential limit at almost all points of $\partial \Omega$.

Theorem 5.1 Let $\Omega_{+} \subset \mathbb{R}^{n}(n \geq 3)$ be a bounded Lipschitz domain with connected boundary $\partial \Omega_{+}$. Let $\alpha \in(0, \infty), p \in(1, \infty)$, and $p^{*}:=\max \{p, 2\}$

(i) Let $\mathbf{h}_{0} \in H_{p ; \nu}^{1}\left(\partial \Omega, \mathbb{R}^{n}\right)$. Then there exists $\varepsilon=\varepsilon(\partial \Omega)>0$ such that for any $p \in \mathcal{R}_{0}(n, \varepsilon)$, the Dirichlet problem (5.1)-(5.2) has a solution $(\mathbf{u}, \pi)$ such that $M(\mathbf{u}), M(\nabla \mathbf{u}), M(\pi) \in L_{p}(\partial \Omega)$ and there exist the non-tangential limits of $\mathbf{u}, \nabla \mathbf{u}$ and $\pi$ at almost all points of the boundary $\partial \Omega$. Moreover, there exists a constant $C=C(\partial \Omega, p, \alpha)>0$ such that

$$
\begin{aligned}
& \|M(\mathbf{u})\|_{L_{p}(\partial \Omega)}+\|M(\nabla \mathbf{u})\|_{L_{p}(\partial \Omega)}+\|M(\pi)\|_{L_{p}(\partial \Omega)} \leq C\left\|\mathbf{h}_{0}\right\|_{H_{p}^{1}\left(\partial \Omega, \mathbb{R}^{n}\right)}, \\
& \left\|\mathbf{u}_{\mathrm{nt}}^{+}\right\|_{L_{p}(\partial \Omega)}+\left\|\nabla \mathbf{u}_{\mathrm{nt}}^{+}\right\|_{L_{p}(\partial \Omega)}+\left\|\pi_{\mathrm{nt}}^{+}\right\|_{L_{p}(\partial \Omega)} \leq C\left\|\mathbf{h}_{0}\right\|_{H_{p}^{1}\left(\partial \Omega, \mathbb{R}^{n}\right)} .
\end{aligned}
$$

In addition, $\mathbf{u} \in B_{p, p^{*}}^{1+\frac{1}{p}}\left(\Omega_{+}, \mathbb{R}^{n}\right), \pi \in B_{p, p^{*}}^{\frac{1}{p}}\left(\Omega_{+}\right)$and

$$
\|\mathbf{u}\|_{B_{p, p^{*}}^{1+\frac{1}{p}}\left(\Omega_{+}, \mathbb{R}^{n}\right)}+\|\pi\|_{B_{p, p^{*}}^{\frac{1}{p}}\left(\Omega_{+}\right)} \leq C\left\|\mathbf{h}_{0}\right\|_{H_{p}^{1}\left(\partial \Omega, \mathbb{R}^{n}\right)}
$$


(ii) Let $\mathbf{h}_{0} \in L_{p ; \nu}\left(\partial \Omega, \mathbb{R}^{n}\right)$. Then there exists $\varepsilon=\varepsilon(\partial \Omega)>0$ such that for any $p \in \mathcal{R}_{1}(n, \varepsilon)$ the Dirichlet problem (5.1)-(5.2) has a solution $(\mathbf{u}, \pi)$ such that $M(\mathbf{u}) \in L_{p}(\partial \Omega)$. Moreover, there exists a constant $C>0$ such that

$$
\|M(\mathbf{u})\|_{L_{p}(\partial \Omega)} \leq C\left\|\mathbf{h}_{0}\right\|_{L_{p}\left(\partial \Omega, \mathbb{R}^{n}\right)} .
$$

In addition, $\mathbf{u} \in B_{p, p^{*}}^{\frac{1}{p}}\left(\Omega_{+}, \mathbb{R}^{n}\right)$ and

$$
\|\mathbf{u}\|_{B_{p, p^{*}}^{\frac{1}{p}}\left(\Omega_{+}, \mathbb{R}^{n}\right)} \leq C\left\|\mathbf{h}_{0}\right\|_{L_{p}\left(\partial \Omega, \mathbb{R}^{n}\right)} .
$$

(iii) Let $0<s<1$ and $\mathbf{h}_{0} \in H_{p ; \nu}^{s}\left(\partial \Omega, \mathbb{R}^{n}\right)$. Then there exists $\varepsilon=\varepsilon(\partial \Omega)>0$ such that for any $p \in \mathcal{R}_{1-s}(n, \epsilon)(c f$. (4.5)), the Dirichlet problem (5.1)-(5.2) (where the Dirichlet condition (5.2) is considered in the Gagliardo trace sense) has a solution $\mathbf{u} \in B_{p, p^{*}}^{s+\frac{1}{p}}\left(\Omega_{+}, \mathbb{R}^{n}\right), \pi \in B_{p, p^{*}}^{s+\frac{1}{p}-1}\left(\Omega_{+}\right)$, and there exists a constant $C>0$ such that

$$
\|\mathbf{u}\|_{B_{p, p^{*}}^{s+\frac{1}{p}}\left(\Omega_{+}, \mathbb{R}^{n}\right)}+\|\pi\|_{B_{p, p^{*}}^{s+\frac{1}{p}-1}\left(\Omega_{+}\right)} \leq C\left\|\mathbf{h}_{0}\right\|_{H_{p}^{s}\left(\partial \Omega, \mathbb{R}^{n}\right)} .
$$

In each of the cases (i), (ii) and (iii), the solution is unique up to an arbitrary additive constant for the pressure $\pi$, and can be expressed in terms of the following double layer velocity and pressure potentials

$$
\mathbf{u}=\mathbf{W}_{\alpha}\left(\left(-\frac{1}{2} \mathbf{I}+\mathbf{K}_{\alpha}\right)^{-1} \mathbf{h}_{0}\right), \pi=\mathcal{Q}_{\alpha}^{d}\left(\left(-\frac{1}{2} \mathbf{I}+\mathbf{K}_{\alpha}\right)^{-1} \mathbf{h}_{0}\right) \text { in } \Omega_{+} .
$$

Proof. According to Lemmas 3.4, 4.4 and Theorem 3.5(iii), the functions given by (5.6) provide a solution of the Dirichlet problem (5.1)-(5.2), which satisfies the corresponding norm estimates mentioned in items (i) - (ii). For $0<s<1$ in item (iii), we have by Corollary 4.5 that $\left(-\frac{1}{2} \mathbf{I}+\mathbf{K}_{\alpha}\right)^{-1} \mathbf{h}_{0} \in H_{p}^{s}\left(\partial \Omega, \mathbb{R}^{n}\right) \hookrightarrow B_{p, p^{*}}^{s}\left(\partial \Omega, \mathbb{R}^{n}\right)$ with corresponding norm estimates, which by (3.40), (3.75) and (3.82) proves the desired solution properties.

We will now prove uniqueness of the solution of the Dirichlet problem (5.1)-(5.2) satisfying the conditions in item (ii), by modifying arguments in the proofs of [61, Theorem 5.5.4] and [62, Theorem 7.1]. Let $\left(\mathbf{u}^{0}, \pi^{0}\right)$ be a solution of the homogeneous version of the Dirichlet problem (5.1)-(5.2) such that $M\left(\mathbf{u}^{0}\right) \in L_{p}(\partial \Omega)$ and $\mathbf{u}_{0}$ satisfies the homogeneous boundary condition in the sense of non-tangential limit at almost all points of the boundary $\partial \Omega$. Let $\mathbf{x}_{0} \in \Omega_{+}$and let $\left\{\Omega_{j}\right\}_{j \geq 1}$ be a sequence of $C^{\infty}$ sub-domains in $\Omega_{+}$that contain $\mathbf{x}_{0}$ and converge to $\Omega_{+}$in the sense described in Lemma 2.2. Let $\mathbf{G}_{k}^{\alpha}(\mathbf{x})=\left(\mathcal{G}_{k 1}^{\alpha}(\mathbf{x}), \ldots, \mathcal{G}_{k n}^{\alpha}(\mathbf{x})\right)$, $k=1, \ldots, n$, where $\left(\mathcal{G}^{\alpha}, \Pi\right)$ is the fundamental solution of the Brinkman system in $\mathbb{R}^{n}$ (see (3.1) and (3.2)). Then for each $\Omega_{j}$ and any $k=1, \ldots, n$, the functions $\mathbf{v}^{j}$ and $q^{j}$ given by

$$
\mathbf{v}_{\mathbf{x}_{0}}^{j}=\mathbf{W}_{\alpha}^{j}\left(\mathbf{h}^{\prime(j)}\right), q_{x_{0}}^{j}=\mathcal{Q}_{\alpha}^{j ; d}\left(\mathbf{h}^{\prime(j)}\right) \text { in } \mathbb{R}^{n} \backslash \partial \Omega_{j}, \quad \mathbf{h}^{\prime(j)}=\left(-\frac{1}{2} \mathbf{I}+\mathbf{K}_{\alpha}^{j}\right)^{-1}\left(\left.\mathbf{G}_{k}^{\alpha}\left(\mathbf{x}_{0}-\cdot\right)\right|_{\partial \Omega_{j}}\right),
$$

satisfy the system

$$
\left\{\begin{array}{l}
\Delta \mathbf{v}_{\mathrm{x}_{0}}^{j}-\alpha \mathbf{v}_{\mathrm{x}_{0}}^{j}-\nabla q_{\mathrm{x}_{0}}^{j}=\mathbf{0}, \operatorname{div} \mathbf{v}_{\mathrm{x}_{0}}^{j}=0 \text { in } \Omega_{j}, \\
\left(\mathbf{v}_{\mathrm{x}_{0}}^{j}\right)_{\mathrm{nt}}^{+}=\left.\mathbf{G}_{k}^{\alpha}\left(\mathbf{x}_{0}, \cdot\right)\right|_{\partial \Omega_{j}} .
\end{array}\right.
$$

Here $\mathbf{W}_{\alpha}^{j}:=\mathbf{W}_{\alpha ; \partial \Omega_{j}}$ and $\mathcal{Q}_{\alpha}^{j ; d}:=\mathcal{Q}_{\alpha ; \partial \Omega_{j}}^{d}$ are the double layer velocity and pressure potential operators corresponding to $\partial \Omega_{j}$, while $\mathbf{K}_{\alpha}^{j}: H_{p^{\prime}}^{1}\left(\partial \Omega_{j}, \mathbb{R}^{n}\right) \rightarrow H_{p^{\prime}}^{1}\left(\partial \Omega_{j}, \mathbb{R}^{n}\right)$ is the corresponding double layer integral operator. Indeed, $\left.\mathbf{G}_{k}^{\alpha}\left(\mathbf{x}_{0}-\cdot\right)\right|_{\partial \Omega_{j}} \in H_{p ; \nu^{(j)}}^{1}\left(\partial \Omega_{j}, \mathbb{R}^{n}\right)$ and, in view of Lemma 4.4, the operator $-\frac{1}{2} \mathbf{I}+\mathbf{K}_{\alpha}^{j}: H_{p^{\prime} ; \boldsymbol{\nu}^{(j)}}^{1}\left(\partial \Omega_{j}, \mathbb{R}^{n}\right) \rightarrow H_{p^{\prime} ; \boldsymbol{\nu}^{(j)}}^{1}\left(\partial \Omega_{j}, \mathbb{R}^{n}\right)$ is an isomorphism for any $p^{\prime} \in(1, \infty)$ since $\Omega_{j}$ is a smooth domain.

Note that the operator $-\frac{1}{2} \mathbf{I}+\mathbf{K}_{\alpha}: H_{p^{\prime} ; \nu}^{1}\left(\partial \Omega, \mathbb{R}^{n}\right) \rightarrow H_{p^{\prime} ; \nu}^{1}\left(\partial \Omega, \mathbb{R}^{n}\right)$ is an isomorphism for any $p^{\prime} \in \mathcal{R}_{0}(n, \varepsilon)$ (see Lemma 4.4), i.e., for any $p^{\prime}$ such that $\frac{1}{p^{\prime}}=1-\frac{1}{p}$, where $p \in \mathcal{R}_{1}(n, \varepsilon)$. After performing a change of variable as in Lemma 2.2, the operator $-\frac{1}{2} \mathbf{I}+\mathbf{K}_{\alpha}^{j}$ defined on $\partial \Omega_{j}$ can be identified with an operator $\mathcal{T}_{\alpha}^{j}$ acting on functions defined on $\partial \Omega$. Then, employing the arguments, e.g., similar to those in the last paragraph in p.116 in [61], which are based on [61, Lemmas 11.9.13 and 11.12.2], and taking into account [47, Proposition 1] (see also [23, Theorems 3.8 (iv) and 4.15]), one can show that the sequence of operators $\mathcal{T}_{\alpha}^{j}$ converges to the operator $\mathcal{T}_{\alpha}:=-\frac{1}{2} \mathbf{I}+\mathbf{K}_{\alpha}$ in the operator norm and the sequence of the inverses of the operators $\mathcal{T}_{\alpha}^{j}$ converges to the inverse of the operator $\mathcal{T}_{\alpha}$ in the operator norm. Hence the operator norms $\left\|\left(-\frac{1}{2} \mathbb{I}+\mathbf{K}_{\alpha}^{j}\right)^{-1}\right\|_{H_{p^{\prime}}^{1}\left(\partial \Omega_{j}, \mathbb{R}^{n}\right)}$ are bounded uniformly in $j$, implying that there exist some constants $C_{0}, C_{0}^{\prime}$ depending only on $p, n, \alpha$ and the Lipschitz character of $\Omega_{+}$(thus, $C_{0}$ does not depend on $j$ ) such that

$$
\left\|\mathbf{h}^{\prime(j)}\right\|_{H_{p^{\prime}}^{1}\left(\partial \Omega_{j}, \mathbb{R}^{n}\right)} \leq C_{0}\left\|\mathbf{G}_{k}^{\alpha}\left(\mathbf{x}_{0}, \cdot\right)\right\|_{H_{p^{\prime}}^{1}\left(\partial \Omega_{j}, \mathbb{R}^{n}\right)} \leq C_{0}^{\prime}\left(\left\|M\left(\mathbf{G}_{k}^{\alpha}\left(\mathbf{x}_{0}, \cdot\right)\right)\right\|_{L_{p^{\prime}}(\partial \Omega)}+\left\|M\left(\nabla \mathbf{G}_{k}^{\alpha}\left(\mathbf{x}_{0}, \cdot\right)\right)\right\|_{L_{p^{\prime}}(\partial \Omega)}\right)
$$

where the non-tangential maximal operator $M$ is considered with respect to a regular family of cones truncated at a height smaller than the distance from $\mathbf{x}_{0}$ to $\partial \Omega$ (cf. [75, Theorem 1.12], see also Lemma 2.2). Further, by considering the change of variable $\mathbf{y}_{j}:=\Phi_{j}(\mathbf{y})$ as in Lemma 2.2, the double-layer potential representations (5.7) become

$$
v_{x_{0} ; \ell}^{j}(\mathbf{x})=\int_{\partial \Omega_{j}} S_{i \ell s}^{\alpha}\left(\mathbf{y}_{j}, \mathbf{x}\right) \nu_{s}\left(\mathbf{y}_{j}\right) h_{i}^{\prime(j)}\left(\mathbf{y}_{j}\right) d \sigma_{y_{j}}=\int_{\partial \Omega} S_{i \ell s}^{\alpha}\left(\Phi_{j}(\mathbf{y}), \mathbf{x}\right) \nu_{s}\left(\Phi_{j}(\mathbf{y})\right) H_{i}^{(j)}(\mathbf{y}) d \sigma_{\mathrm{y}},
$$




$$
q_{\mathrm{x}_{0}}^{j}(\mathbf{x})=\int_{\partial \Omega_{j}} \Lambda_{i s}^{\alpha}\left(\mathbf{y}_{j}, \mathbf{x}\right) \nu_{s}\left(\mathbf{y}_{j}\right) h_{i}^{\prime(j)}\left(\mathbf{y}_{j}\right) d \sigma_{y_{j}}=\int_{\partial \Omega} \Lambda_{i s}^{\alpha}\left(\Phi_{j}(\mathbf{y}), \mathbf{x}\right) \nu_{s}\left(\Phi_{j}(\mathbf{y})\right) H_{i}^{\prime(j)}(\mathbf{y}) d \sigma_{y}, \forall \mathbf{x} \in \Omega_{j}
$$

where $\mathbf{H}^{\prime(j)}(\mathbf{y}):=\mathbf{h}^{\prime(j)}\left(\Phi_{j}(\mathbf{y})\right) \omega_{j}(\mathbf{y}), \mathbf{y} \in \partial \Omega, \mathbf{y}^{(j)}=\left(y_{1}^{(j)}, \ldots, y_{n}^{(j)}\right), \mathbf{h}^{\prime(j)}=\left(h_{1}^{\prime(j)}, \ldots, h_{n}^{\prime(j)}\right), \mathbf{H}^{\prime(j)}=\left({H^{\prime}}_{1}^{(j)}, \ldots,{H^{\prime}}_{n}^{(j)}\right)$, and $\omega_{j}$ is the Jacobian of $\Phi_{j}: \partial \Omega \rightarrow \partial \Omega_{j}$.

In view of (5.9) and of the uniform boundedness of $\left\{\omega_{j}\right\}_{j \geq 1}$, there exists a constant $C_{1}>0$ (which depends only on $p, n$ and the Lipschitz character of $\Omega_{+}$) such that

$$
\left\|\mathbf{H}^{\prime(j)}\right\|_{H_{p^{\prime}}^{1}\left(\partial \Omega, \mathbb{R}^{n}\right)} \leq C_{1}\left\|\mathbf{h}^{\prime(j)}\right\|_{H_{p^{\prime}}^{1}\left(\partial \Omega_{j}, \mathbb{R}^{n}\right)} \leq C_{0}^{\prime} C_{1}\left(\left\|M\left(\mathbf{G}_{k}^{\alpha}\left(\mathbf{x}_{0}, \cdot\right)\right)\right\|_{L_{p^{\prime}}(\partial \Omega)}+\left\|M\left(\nabla \mathbf{G}_{k}^{\alpha}\left(\mathbf{x}_{0}, \cdot\right)\right)\right\|_{L_{p^{\prime}}(\partial \Omega)}\right), \quad \forall j \geq 1 .
$$

Hence $\left\{\mathbf{H}^{\prime(j)}\right\}_{j \geq 1}$ is a bounded sequence in $H_{p^{\prime}}^{1}\left(\partial \Omega, \mathbb{R}^{n}\right)$, and, thus, there exists a subsequence, still denoted as the sequence, and a function $\mathbf{H}^{\prime} \in H_{p^{\prime}}^{1}\left(\partial \Omega, \mathbb{R}^{n}\right)$, such that $\mathbf{H}^{\prime(j)} \rightarrow \mathbf{H}^{\prime}$ weakly in $H_{p^{\prime}}^{1}\left(\partial \Omega, \mathbb{R}^{n}\right)$. By this property and letting $j \rightarrow \infty$ in $(5.10)-(5.11)$, we obtain $\mathbf{v}_{\mathrm{x}_{0}}^{j}(\mathbf{x}) \rightarrow \mathbf{v}_{\mathrm{x}_{0}}(\mathbf{x})=\mathbf{W}_{\alpha} \mathbf{H}^{\prime}(\mathbf{x}), q_{\mathrm{x}_{0}}^{j}(\mathbf{x}) \rightarrow q_{\mathrm{x}_{0}}(\mathbf{x})=\mathcal{Q}_{\alpha}^{d} \mathbf{H}^{\prime}(\mathbf{x})$ pointwise for any $\mathbf{x} \in \Omega_{+}$. Moreover, in view of Lemma 3.4 (where the constants depend only on the Lipschitz character of $\Omega_{+}$), applied to $\partial \Omega_{j}$, and (5.9), we obtain the inequality

$$
\left\|M\left(\nabla \mathbf{v}_{\mathbf{x}_{0}}^{j}\right)\right\|_{L_{p^{\prime}}\left(\partial \Omega_{j}\right)}+\left\|M\left(q_{x_{0}}^{j}\right)\right\|_{L_{p^{\prime}}\left(\partial \Omega_{j}\right)} \leq C_{3}\left\|\mathbf{h}^{\prime(j)}\right\| \leq C_{0}^{\prime} C_{3}\left(\left\|M\left(\mathbf{G}_{k}^{\alpha}\left(\mathbf{x}_{0}, \cdot\right)\right)\right\|_{L_{p^{\prime}}(\partial \Omega)}+\left\|M\left(\nabla \mathbf{G}_{k}^{\alpha}\left(\mathbf{x}_{0}, \cdot\right)\right)\right\|_{L_{p^{\prime}}(\partial \Omega)}\right),
$$

with a constant $C_{3}$ depending only on $p, n$ and the Lipschitz character of $\Omega_{+}$.

In addition, the pair $\left(\mathbf{G}_{k}^{\alpha ; j}\left(\mathbf{x}_{0}, \cdot\right), \pi_{k}^{j}\left(\mathbf{x}_{0}, \cdot\right)\right)$ given by

$$
\mathbf{G}_{k}^{\alpha ; j}\left(\mathbf{x}_{0}, \cdot\right):=\mathbf{G}_{k}^{\alpha}\left(\mathbf{x}_{0}-\cdot\right)-\mathbf{v}_{\mathbf{x}_{0}}^{j}, \quad \pi_{k}^{j}\left(\mathbf{x}_{0}, \cdot\right):=\Pi_{k}\left(\mathbf{x}_{0}-\cdot\right)-q_{\mathbf{x}_{0}}^{j}
$$

defines the Green function of the Brinkman system in $\Omega_{j}$ and its corresponding pressure vector, i.e., it satisfies for each $\mathbf{x}_{0} \in \Omega_{j}$ the following relations

$$
\left\{\begin{array}{l}
-\nabla \pi_{k}^{j}\left(\mathbf{x}_{0}, \mathbf{y}\right)+\triangle \mathbf{G}_{k}^{\alpha ; j}\left(\mathbf{x}_{0}, \mathbf{y}\right)-\alpha \mathbf{G}_{k}^{\alpha: j}\left(\mathbf{x}_{0}, \mathbf{y}\right)=-\delta_{\mathbf{y}}\left(\mathbf{x}_{0}\right) \mathbf{I} \\
\operatorname{div}_{\mathbf{y}} \mathbf{G}_{k}^{\alpha ; j}\left(\mathbf{x}_{0}, \mathbf{y}\right)=0 \text { in } \Omega_{j} \\
\mathbf{G}_{k}^{\alpha ; j}\left(\mathbf{x}_{0}, \mathbf{y}\right)=\mathbf{0}, \mathbf{y} \in \partial \Omega_{j} .
\end{array}\right.
$$

Hence, for each $\Omega_{j}$ and any $k=1, \ldots, n$, we obtain the relations

$$
\left\langle\triangle \mathbf{G}_{k}^{\alpha ; j}\left(\mathbf{x}_{0}, \cdot\right)-\alpha \mathbf{G}_{k}^{\alpha ; j}\left(\mathbf{x}_{0}, \cdot\right)-\nabla \pi_{k}^{j}\left(\mathbf{x}_{0}, \cdot\right), \mathbf{u}^{0}\right\rangle_{\Omega_{j}}=u_{k}^{0}\left(\mathbf{x}_{0}\right) .
$$

Then by (5.15) and (5.16) we obtain that

$$
u_{k}^{0}\left(\mathbf{x}_{0}\right)=\int_{\partial \Omega_{j}} \mathbf{t}^{c+}\left(\mathbf{G}_{k}^{\alpha ; j}\left(\mathbf{x}_{0}, \cdot\right), \pi_{k}^{j}\left(\mathbf{x}_{0}, \cdot\right)\right) \cdot \mathbf{u}^{0} d \sigma_{j} .
$$

By (5.14) and (5.13), there exists a constant $C$ depending only on $\alpha, p, n$ and the Lipschitz character of $\Omega_{+}$such that

$$
\left\|M\left(\nabla \mathbf{G}_{k}^{\alpha ; j}\left(\mathbf{x}_{0}, \cdot\right)\right)\right\|_{L_{p^{\prime}}\left(\partial \Omega_{j}\right)}+\left\|M\left(\pi_{k}^{j}\left(\mathbf{x}_{0}, \cdot\right)\right)\right\|_{L_{p^{\prime}}\left(\partial \Omega_{j}\right)} \leq C\left(\left\|M\left(\mathbf{G}_{k}^{\alpha}\left(\mathbf{x}_{0}, \cdot\right)\right)\right\|_{L_{p^{\prime}}(\partial \Omega)}+\left\|M\left(\nabla \mathbf{G}_{k}^{\alpha}\left(\mathbf{x}_{0}, \cdot\right)\right)\right\|_{L_{p^{\prime}}(\partial \Omega)}\right)
$$

Since also $M\left(\mathbf{u}^{0}\right) \in L_{p}(\partial \Omega)$ and $\left(\mathbf{u}^{0}\right)_{\text {nt }}^{+}=\mathbf{0}$ on $\partial \Omega$, then the Lebesgue Dominated Convergence Theorem (applied again after the change of variable as in Lemma 2.2 that reduces the integral over $\partial \Omega_{j}$ to an integral over $\partial \Omega$ ) implies that the right hand side in (5.17) tends to zero as $\partial \Omega_{j}$ tends to $\partial \Omega$ and hence $u_{k}^{0}\left(\mathbf{x}_{0}\right)=0$. Because $\mathbf{x}_{0}$ is an arbitrary point in $\Omega_{+}$, we conclude that $\mathbf{u}^{0}=\mathbf{0}$ in $\Omega_{+}$, and by the first equation in (5.1), $\pi^{0}$ is a constant pressure, as asserted. This completes the proof of the uniqueness in item (ii).

Let us show also the uniqueness result for item $(i)$. To do so, assume that $\left(\mathbf{u}_{0}, \pi_{0}\right)$ is a solution of the homogeneous version of the Dirichlet problem (5.1) such that $M\left(\mathbf{u}_{0}\right), M\left(\nabla \mathbf{u}_{0}\right), M\left(\pi_{0}\right) \in L_{p}(\partial \Omega)$, there exist the non-tangential limits of $\mathbf{u}_{0}, \nabla \mathbf{u}_{0}$ and $\pi_{0}$ at almost all points of the boundary $\partial \Omega$, and $\mathbf{u}_{0}$ satisfies the homogeneous Dirichlet boundary condition in the sense of non-tangential limit at almost all points of $\partial \Omega$. Then the Green representation formula $\mathbf{u}_{0}=\mathbf{V}_{\alpha}\left(\mathbf{t}_{\mathrm{nt}}^{+}\left(\mathbf{u}_{0}, \pi_{0}\right)\right)-\mathbf{W}_{\alpha}\left(\mathbf{u}_{\mathrm{nt}}^{0+}\right)$ in $\Omega_{+}$(cf. Lemma 3.8) reduces to $\mathbf{u}_{0}=\mathbf{V}_{\alpha}\left(\mathbf{t}_{\mathrm{nt}}^{+}\left(\mathbf{u}_{0}, \pi_{0}\right)\right)$ in $\Omega_{+}$, and, by considering the non-tangential trace, we obtain that $\mathcal{V}_{\alpha}\left(\mathbf{t}_{\mathrm{nt}}^{+}\left(\mathbf{u}_{0}, \pi_{0}\right)\right)=\mathbf{0}$ on $\partial \Omega$. Thus, $\mathbf{t}_{\mathrm{nt}}^{+}\left(\mathbf{u}_{0}, \pi_{0}\right) \in \mathbb{R} \boldsymbol{\nu}$ (see Lemma 4.6), and hence $\mathbf{u}_{0}=\mathbf{0}$ in $\Omega_{+}$, while the Brinkman equation (5.1) shows that $\pi^{0}=0$ in $\Omega_{+}$(up to an additive constant pressure). This completes the proof of the statement in item (i).

Next we show for $s \in(0,1)$ the uniqueness of a solution to the Dirichlet problem (5.1)-(5.2), in the hypothesis of item (iii). To this end, let $\left(\mathbf{u}^{0}, \pi^{0}\right) \in B_{p, p^{*}}^{s+\frac{1}{p}}\left(\Omega_{+}, \mathbb{R}^{n}\right) \times B_{p, p^{*}}^{s+\frac{1}{p}}\left(\Omega_{+}\right)$denote a solution of the homogeneous version of the Dirichlet problem (5.1)-(5.2). By Lemmas 2.4, 2.11 and Theorem 2.5 we obtain that $\gamma_{+} \mathbf{u}^{0}=\mathbf{u}_{\mathrm{nt}}^{0+}=0$ and $\mathbf{t}_{\alpha}^{+}\left(\mathbf{u}^{0}, \pi^{0}\right) \in B_{p, p^{*}}^{s-1}\left(\partial \Omega, \mathbb{R}^{n}\right)$. Then for $s \in(0,1)$, the Green representation formula (3.128) applied to the pair $\left(\mathbf{u}^{0}, \pi^{0}\right)$ implies that $\gamma_{+} \mathbf{V}_{\alpha}\left(\mathbf{t}_{\alpha}^{+}(\mathbf{u}, \pi)\right)=\mathbf{0}$ on $\partial \Omega$. Hence by (3.88) and (4.29) we obtain that $\mathbf{t}_{\alpha}^{+}(\mathbf{u}, \boldsymbol{\pi}) \in \mathbb{R} \boldsymbol{\nu}$. Since $\mathbf{V}_{\alpha} \boldsymbol{\nu}=\mathbf{0}$ in $\Omega_{+}$, we deduce that $\mathbf{u}_{0}=\mathbf{0}$ in $\Omega_{+}$, and by the Brinkman equation (5.1) $\pi^{0}=0$ (up to an additive constant).

Note that for $p=2$, Theorem 5.1 (ii) has been obtained by Z. Shen in [69, Theorem 5.5] by using another double layer potential approach.

The following regularity result has been obtained in [61, Theorem 4.3.1] and [62, Theorem 7.1] in the case of the Stokes system (i.e., for $\alpha=0$ ). We prove a similar result in the case of the Brinkman system (i.e., for $\alpha>0$ ) by using the main ideas of the proof of [62, Theorem 7.1] (see also [56, (2.95), Remark V p. 37], [16, Theorem 2], [35, Lemma 3.3], [45]). 
Theorem 5.2 Let $\Omega_{+} \subset \mathbb{R}^{n}$ be a bounded Lipschitz domain with connected boundary $\partial \Omega$. Let $\alpha \geq 0, p \in(1, \infty)$ and $p^{*}:=$ $\max \{p, 2\}$. Assume that a pair $(\mathbf{u}, \pi)$ satisfies the homogeneous Brinkman system (5.1). Then the following properties hold.

(i) There exists $\varepsilon=\varepsilon(\partial \Omega)>0$ such that for any $p \in(2-\varepsilon, \infty)$, the condition $M(\mathbf{u}) \in L_{p}(\partial \Omega)$ implies that there exists the non-tangential limit of $\mathbf{u}$ almost everywhere on $\partial \Omega$ and $\mathbf{u}_{\mathrm{nt}}^{+} \in L_{p ; \nu}\left(\partial \Omega, \mathbb{R}^{n}\right)$. Moreover,

$$
\left\|\mathbf{u}_{\mathrm{nt}}^{+}\right\|_{L_{p}\left(\partial \Omega, \mathbb{R}^{n}\right)} \leq C_{1}\|M(\mathbf{u})\|_{L_{p}(\partial \Omega)}, \quad\|\mathbf{u}\|_{B_{p, p^{*}}^{\frac{1}{p}}\left(\Omega_{+}, \mathbb{R}^{n}\right)} \leq C_{1}^{\prime}\|M(\mathbf{u})\|_{L_{p}(\partial \Omega)},
$$

with some constants $C_{1} \equiv C_{1}(\partial \Omega, p, \alpha)>0, C_{1}^{\prime} \equiv C_{1}^{\prime}(\partial \Omega, p, \alpha)>0$.

(ii) There exists $\varepsilon=\varepsilon(\partial \Omega)>0$ such that for any $p \in \mathcal{R}_{0}(n, \varepsilon) \cup(2, \infty)$, the assumption $M(\mathbf{u}), M(\nabla \mathbf{u}), M(\pi) \in L_{p}(\partial \Omega)$ implies that there exist the non-tangential limits of $\mathbf{u}, \nabla \mathbf{u}, \pi$ almost everywhere on $\partial \Omega$, and that $\mathbf{u}_{n t}^{+} \in H_{p: \nu}^{1}\left(\partial \Omega, \mathbb{R}^{n}\right)$ and $\mathbf{t}_{\mathrm{nt}}^{+}(\mathbf{u}, \pi) \in L_{p}\left(\partial \Omega, \mathbb{R}^{n}\right)$. In addition, there exist some constants $C_{2} \equiv C_{2}(\partial \Omega, p, \alpha)>0, C_{2}^{\prime} \equiv C_{2}^{\prime}(\partial \Omega, p, \alpha)>0$ such that

$$
\begin{aligned}
&\left\|\mathbf{u}_{\mathrm{nt}}^{+}\right\|_{H_{p}^{1}\left(\partial \Omega, \mathbb{R}^{n}\right)}+\left\|\mathbf{t}_{\mathrm{nt}}^{+}(\mathbf{u}, \pi)\right\|_{L_{p}\left(\partial \Omega, \mathbb{R}^{n}\right)} \leq C_{2}\left(\|M(\mathbf{u})\|_{L_{p}(\partial \Omega)}+\|M(\nabla \mathbf{u})\|_{L_{p}(\partial \Omega)}+\|M(\pi)\|_{L_{p}(\partial \Omega)}\right), \\
&\|\mathbf{u}\|_{\substack{B_{p, p^{*}}^{1+\frac{1}{p}}\left(\Omega_{+}, \mathbb{R}^{n}\right)\\
}}+\|\pi\|_{B_{p, p^{*}}^{\frac{1}{p}}\left(\Omega_{+}\right)} \leq C_{2}^{\prime}\left(\|M(\mathbf{u})\|_{L_{p}(\partial \Omega)}+\|M(\nabla \mathbf{u})\|_{L_{p}(\partial \Omega)}+\|M(\pi)\|_{L_{p}(\partial \Omega)}\right) .
\end{aligned}
$$

Proof. (i) We will use arguments similar to the ones in the proof of [16, Lemma 8]. First, let $\left\{\Omega_{j}\right\}_{j \geq 1}$ be a sequence of subdomains in $\Omega_{+}$that converge to $\Omega_{+}$in the sense described in Lemma 2.2, with the corresponding notations $\Phi_{j}, \boldsymbol{\nu}^{(j)}$ and $\omega_{j}$ also introduced there. Due to ellipticity of the homogeneous Brinkman system in $\Omega_{+}$, we have $(\mathbf{u}, \pi) \in C^{\infty}\left(\Omega_{+}, \mathbb{R}^{n}\right) \times C^{\infty}\left(\Omega_{+}\right)$. Now, let $\mathbf{h}^{(j)}:=\left.\mathbf{u}\right|_{\partial \Omega_{j}}$. Then $\left(\mathbf{u}_{j}, \pi_{j}\right):=\left(\left.\mathbf{u}\right|_{\Omega_{j}},\left.\pi\right|_{\Omega_{j}}\right)$ satisfies the homogeneous Brinkman system in $\Omega_{j}$ and the Dirichlet boundary condition $\left.\mathbf{u}_{j}\right|_{\partial \Omega_{j}}=\mathbf{h}^{(j)}$ on $\partial \Omega_{j}$, where $\mathbf{h}^{(j)} \in L_{p ; \boldsymbol{\nu}}(j)\left(\partial \Omega_{j}, \mathbb{R}^{n}\right)$. The solution of such a problem is unique, up to an additive constant for the pressure (see, e.g., Theorem 5.1).

According to Lemma 4.4 applied to the smooth domain $\Omega_{j}$, such a solution can be expressed in terms of the double layer potential $\mathbf{u}_{j}=\mathbf{W}_{\alpha ; \partial \Omega_{j}} \mathbf{h}^{(j)}, \pi_{j}=\mathcal{Q}_{\alpha ; \partial \Omega_{j}}^{d} \mathbf{h}^{\prime(j)}$, with a density $\mathbf{h}^{\prime(j)} \in L_{p: \boldsymbol{\nu}^{(j)}}\left(\partial \Omega_{j}, \mathbb{R}^{n}\right)$ satisfying the equation $\left(-\frac{1}{2} \mathbb{I}+\mathbf{K}_{\alpha}^{j}\right) \mathbf{h}^{\prime(j)}=\mathbf{h}^{(j)}$, where $\mathbf{K}_{\alpha}^{j}:=\mathbf{K}_{\alpha ; \partial \Omega_{j}}$ is associated (as in (3.89)) with the double layer potential $W_{\alpha ; \partial \Omega_{j}}$ defined on $L_{p: \nu}(j)\left(\partial \Omega_{j}, \mathbb{R}^{n}\right)$, and, in view of Lemma 4.4, the operator $-\frac{1}{2} \mathbf{I}+\mathbf{K}_{\alpha}^{j}: L_{p ; \boldsymbol{\nu}^{(j)}}\left(\partial \Omega_{j}, \mathbb{R}^{n}\right) \rightarrow L_{p ; \boldsymbol{\nu}^{(j)}}\left(\partial \Omega_{j}, \mathbb{R}^{n}\right)$ is an isomorphism for any $p \in(1, \infty)$.

Note that the operator $-\frac{1}{2} \mathbf{I}+\mathbf{K}_{\alpha}: L_{p ; \boldsymbol{\nu}}\left(\partial \Omega, \mathbb{R}^{n}\right) \rightarrow L_{p ; \boldsymbol{\nu}}\left(\partial \Omega, \mathbb{R}^{n}\right)$ is an isomorphism for any $p \in \mathcal{R}_{1}(n, \varepsilon)$ (see Lemma 4.4$)$. After performing a change of variable as in Lemma 2.2, the operator $-\frac{1}{2} \mathbf{I}+\mathbf{K}_{\alpha}^{j}$ defined on $\partial \Omega_{j}$ can be identified with an operator $\mathcal{T}_{\alpha}^{j}$ acting on functions defined on $\partial \Omega$. Then, employing the arguments, e.g., similar to those in the last paragraph in p.116 in [61], which are based on [61, Lemmas 11.9.13 and 11.12.2], and taking into account [47, Proposition 1] (see also [23, Theorems 3.8 (iv) and 4.15]), one can show that the sequence of operators $\mathcal{T}_{\alpha}^{j}$ converges to the operator $\mathcal{T}_{\alpha}:=-\frac{1}{2} \mathbf{I}+\mathbf{K}_{\alpha}$ in the operator norm and the sequence of the inverses of the operators $\mathcal{T}_{\alpha}^{j}$ converges to the inverse of the operator $\mathcal{T}_{\alpha}$ in the operator norm for $p \in \mathcal{R}_{1}(n, \varepsilon)$. Hence, if $p \in \mathcal{R}_{1}(n, \varepsilon)$, the operator norms $\left\|\left(-\frac{1}{2} \mathbb{I}+\mathbf{K}_{\alpha}^{j}\right)^{-1}\right\|_{L_{p}\left(\partial \Omega_{j}, \mathbb{R}^{n}\right)}$ are bounded uniformly in $j$, implying that there exists a constant $c_{0}$ depending only on $p, n, \alpha$, and the Lipschitz character of $\Omega_{+}$(thus, not depending on $j$ ) such that

$$
\begin{aligned}
\left\|\mathbf{h}^{\prime(j)}\right\|_{L_{p}\left(\partial \Omega_{j}, \mathbb{R}^{n}\right)}^{p} & \leq c_{0}\left\|\mathbf{h}^{(j)}\right\|_{L_{p}\left(\partial \Omega_{j}, \mathbb{R}^{n}\right)}^{p}=c_{0}\|\mathbf{u}\|_{L_{p}\left(\partial \Omega_{j}, \mathbb{R}^{n}\right)}^{p} \\
& =c_{0} \int_{\partial \Omega_{j}}\left|\mathbf{u}\left(\mathbf{y}_{j}\right)\right|^{p} d \sigma_{y_{j}}=c_{0} \int_{\partial \Omega}\left|\mathbf{u}\left(\Phi_{j}(\mathbf{y})\right)\right|^{p} \omega_{j}(\mathbf{y}) d \sigma_{y} \leq c_{1} \int_{\partial \Omega}|M(\mathbf{u}(\mathbf{y}))|^{p} d \sigma_{y}=c_{1}\|M(\mathbf{u})\|_{L_{p}\left(\partial \Omega, \mathbb{R}^{n}\right)}^{p},
\end{aligned}
$$

Recall that we have approximated the domain $\Omega_{+}$with a sequence of smooth domains $\Omega_{j}$ with uniform Lipschitz characters from inside, and we have employed here the change of variable $\mathbf{y}_{j}:=\Phi_{j}(\mathbf{y}), \mathbf{y} \in \partial \Omega, \mathbf{y}_{j} \in \partial \Omega_{j}$, and $\omega_{j}$ is the Jacobian of $\Phi_{j}: \partial \Omega \rightarrow \partial \Omega_{j}$ (cf. Lemma 2.2). Hence the constants $c_{0}$ and $c_{1}$ depend only on $p, n, \alpha$, and the Lipschitz character of $\Omega_{+}$.

Further, the double-layer potential $\mathbf{W}_{\alpha ; \partial \Omega_{j}} \mathbf{h}^{\prime(j)}$ becomes

$$
u_{\ell}(\mathbf{x})=\int_{\partial \Omega_{j}} S_{i \ell s}^{\alpha}\left(\mathbf{y}_{j}, \mathbf{x}\right) \nu_{s}\left(\mathbf{y}_{j}\right) h_{i}^{\prime(j)}\left(\mathbf{y}_{j}\right) d \sigma_{y_{j}}=\int_{\partial \Omega} S_{i \ell s}^{\alpha}\left(\Phi_{j}(\mathbf{y}), \mathbf{x}\right) \nu_{s}\left(\Phi_{j}(\mathbf{y})\right) H_{i}^{\prime(j)}(\mathbf{y}) d \sigma_{\mathrm{y}}, \quad \forall \mathbf{x} \in \Omega_{j}
$$

where $\mathbf{H}^{(j)}(\mathbf{y}):=\mathbf{h}^{\prime(j)}\left(\Phi_{j}(\mathbf{y})\right) \omega_{j}(\mathbf{y}), \mathbf{h}^{(j)}=\left(h_{1}^{\prime(j)}, \ldots, h_{n}^{\prime(j)}\right), \mathbf{H}^{\prime(j)}=\left({H^{\prime}}_{1}^{(j)}, \ldots,{H_{n}^{\prime}}_{n}^{(j)}\right)$.

In view of (5.21) and of the uniform boundedness of $\left\{\omega_{j}\right\}_{j \geq 1}$, there exist some constants $c_{1}, c_{2}>0$ (which depend only on $\Omega_{+}$and $p$ ) such that

$$
\int_{\partial \Omega}\left|\mathbf{H}^{\prime(j)}(\mathbf{y})\right|^{p} d \sigma_{\mathrm{y}} \leq c_{2} \int_{\partial \Omega_{j}}\left|\mathbf{u}\left(\mathbf{y}_{j}\right)\right|^{p} d \sigma_{y_{j}} \leq c_{2}^{\prime} \int_{\partial \Omega}|M(\mathbf{u}(\mathbf{y}))|^{p} d \sigma_{y}, \forall j \geq 1
$$

Hence $\left\{\mathbf{H}^{\prime(j)}\right\}_{j>1}$ is a bounded sequence in $L_{p}\left(\partial \Omega, \mathbb{R}^{n}\right)$, and, thus, there exists a subsequence, still denoted as the sequence, and a function $\mathbf{H}^{\prime} \in L_{p}\left(\partial \Omega, \mathbb{R}^{n}\right)$, such that $\mathbf{H}^{\prime(j)} \rightarrow \mathbf{H}^{\prime}$ weakly in $L_{p}\left(\partial \Omega, \mathbb{R}^{n}\right)$. By this property and letting $j \rightarrow \infty$ in (5.22), we obtain $\mathbf{u}=\mathbf{W}_{\alpha} \mathbf{H}^{\prime}$ in $\Omega_{+}$. According to Lemma 3.4(i,iv), there exists the non-tangential limit $\mathbf{u}_{\mathrm{nt}}^{+}=\left(\mathbf{W}_{\alpha} \mathbf{H}^{\prime}\right)_{\mathrm{nt}}^{+}$of $\mathbf{u}$ at almost all points of $\partial \Omega$, and by estimates (3.40) and (5.23), we obtain that

$$
\left\|\mathbf{u}_{\mathrm{nt}}^{+}\right\|_{L_{p}\left(\partial \Omega, \mathbb{R}^{n}\right)}=\left\|\left(\mathbf{W}_{\alpha} \mathbf{H}^{\prime}\right)_{\mathrm{nt}}^{+}\right\|_{L_{p}\left(\partial \Omega, \mathbb{R}^{n}\right)} \leq c_{3}\left\|\mathbf{H}^{\prime}\right\|_{L_{p}\left(\partial \Omega, \mathbb{R}^{n}\right)} \leq c_{3} \lim _{j \rightarrow \infty} \inf _{j \rightarrow \mathbf{H}^{(j)}}\left\|_{L_{p}\left(\partial \Omega, \mathbb{R}^{n}\right)} \leq c_{4}\right\| M(\mathbf{u}) \|_{L_{p}(\partial \Omega)},
$$


where the constants $c_{3}, c_{4}>0$ do not depend on $j$. Moreover, the divergence theorem shows that $\mathbf{u}_{\mathrm{nt}}^{+}=\left(\mathbf{W}_{\alpha} \mathbf{H}^{\prime}\right)_{\mathrm{nt}}^{+} \in L_{p ; \nu}\left(\partial \Omega, \mathbb{R}^{n}\right)$. Estimate (5.18) is provided by the representation $\mathbf{u}=\mathbf{W}_{\alpha} \mathbf{H}^{\prime}$, by continuity of operator (3.70), and by estimates (5.24). This completes the proof of item (i) for any $p \in \mathcal{R}_{1}(n, \varepsilon)$.

Let us now consider item (i) for any $p>2$ (not covered yet when $n>3$ ). Note that inclusions $2 \in \mathcal{R}_{1}(n, \varepsilon)$ and $L_{p}(\partial \Omega) \subset$ $L_{2}(\partial \Omega)$ particularly imply that for such $p$ there exist non-tangential limits of u almost everywhere on $\partial \Omega$. Implementing now, e.g., [58, Proposition 3.29] completes the proof for any $p>2$.

(ii) Now assume that $\mathbf{u}$ and $\pi$ satisfy the Brinkman system and that $M(\mathbf{u}), M(\nabla \mathbf{u}), M(\pi) \in L_{p}(\partial \Omega)$. As in the proof of item (i), we consider again a sequence of smooth domains $\left\{\Omega_{j}\right\}_{j \in \mathbb{N}}$, such that $\bar{\Omega}_{j} \subseteq \Omega_{+}$and $\Omega_{j} \rightarrow \Omega_{+}$as $j \rightarrow \infty$.

As we already mentioned, $\left(\mathbf{u}_{j}, \pi_{j}\right):=\left(\left.\mathbf{u}\right|_{\bar{\Omega}_{j}},\left.\pi\right|_{\Omega_{j}}\right) \in C^{\infty}\left(\bar{\Omega}_{j}, \mathbb{R}^{n}\right) \times C^{\infty}\left(\bar{\Omega}_{j}\right)$. Thus, $\mathbf{h}^{(j)}:=\left.\mathbf{u}\right|_{\partial \Omega_{j}} \in C^{\infty}\left(\partial \Omega_{j}, \mathbb{R}^{n}\right) \subset H_{p}^{1}\left(\partial \Omega_{j}, \mathbb{R}^{n}\right)$ and $\mathbf{h}^{(j)} \in L_{p ; \boldsymbol{\nu}}(j)\left(\partial \Omega_{j}, \mathbb{R}^{n}\right)$, for any $j \in \mathbb{N}$. Then the pair $\left(\mathbf{u}_{j}, \pi_{j}\right) \in C^{\infty}\left(\Omega_{j}, \mathbb{R}^{n}\right) \times C^{\infty}\left(\Omega_{j}\right)$ satisfies the Brinkman system in $\Omega_{j}$ with the Dirichlet boundary condition $\left.\mathbf{u}_{j}\right|_{\partial \Omega_{j}}=\mathbf{h}^{(j)} \in H_{p ; \boldsymbol{\nu}^{(j)}}^{1}\left(\partial \Omega_{j}, \mathbb{R}^{n}\right)$. The solution of such a problem is unique up to an additive constant pressure (see Theorem 5.1(i)) and can be expressed in terms of a double layer potential as in item (i), but now with a density in $H_{p ; \nu^{(j)}}^{1}\left(\partial \Omega_{j}, \mathbb{R}^{n}\right)$. Proceeding similar to the proof of item (i), we prove item (ii).

Remark 5.3 The condition requiring the existence of the non-tangential limits of $\mathbf{u}, \nabla \mathbf{u}$ and $\pi$ at almost all points of the boundary $\partial \Omega$ in Lemma 3.8 is particularly satisfied if $p \in \mathcal{R}_{0}(n, \varepsilon) \cup(2, \infty)$ with $\varepsilon>0$ as in Theorem 5.2(ii). Indeed, for such $p$, the condition is implied by the inclusions $M(\mathbf{u}), M(\nabla \mathbf{u}), M(\pi) \in L_{p}(\partial \Omega)$ and by the Brinkman system (3.129).

Having in view Theorem 5.1(iii), we are now able to consider the Poisson-Dirichlet problem for the Brinkman system,

$$
\left\{\begin{array}{l}
\triangle \mathbf{u}-\alpha \mathbf{u}-\nabla \pi=\mathbf{f}, \operatorname{div} \mathbf{u}=0 \text { in } \Omega_{+} \\
\gamma_{+} \mathbf{u}=\mathbf{h}_{0} \text { on } \partial \Omega
\end{array}\right.
$$

with the Dirichlet datum for the Gagliardo trace $\gamma_{+} \mathbf{u}$ (see also [61, Theorem 10.6.2] for $\alpha=0$ ).

Theorem 5.4 Let $\Omega_{+} \subset \mathbb{R}^{n}(n \geq 3)$ be a bounded Lipschitz domain with connected boundary $\partial \Omega$. Let $\alpha \in(0, \infty)$ and $0<s \leq 1$. Then there exists $\varepsilon=\varepsilon(\partial \Omega)>0$ such that for any $p \in \mathcal{R}_{1-s}(n, \epsilon)\left(c f\right.$. (4.5)), the Dirichlet problem (5.25) with $\mathbf{f} \in L_{p}\left(\Omega_{+}, \mathbb{R}^{3}\right)$ and $\mathbf{h}_{0} \in H_{p ; \nu}^{s}\left(\partial \Omega, \mathbb{R}^{n}\right)$ has a solution $(\mathbf{u}, \pi) \in B_{p, p^{*}}^{s+\frac{1}{p}}\left(\Omega_{+}, \mathbb{R}^{n}\right) \times B_{p, p^{*}}^{s+\frac{1}{p}-1}\left(\Omega_{+}\right)$, which is unique up to an arbitrary additive constant for the pressure $\pi$, where $p^{*}=\max \{2, p\}$. In addition, there exists a constant $C=C\left(s, p, \Omega_{+}\right)>0$ such that

$$
\|\mathbf{u}\|_{B_{p, p^{*}}^{s+\frac{1}{p}}\left(\Omega_{+}, \mathbb{R}^{n}\right)}+\|\pi\|_{B_{p, p^{*}}^{s+\frac{1}{p}-1}\left(\Omega_{+}\right) / \mathbb{R}} \leq C\left(\|\mathbf{f}\|_{L_{p}\left(\Omega_{+}, \mathbb{R}^{n}\right)}+\left\|\mathbf{h}_{0}\right\|_{H_{p}^{s}\left(\partial \Omega, \mathbb{R}^{n}\right)}\right) .
$$

Proof. If $\mathbf{f}=\mathbf{0}$, the existence of a solution of the problem (5.25) for $0<s<1$ is implied by Theorem 5.1(iii) together with the asserted estimate, while for $s=1$ it follows from Theorems 5.1 (i) and 2.5 (iii).

If $\mathbf{f} \neq \mathbf{0}$, we will look for a solution of problem (5.25) in the form

$$
\mathbf{u}=\mathbf{N}_{\alpha ; \Omega_{+}} \mathbf{f}+\mathbf{v}, \pi=\mathcal{Q}_{\Omega_{+}} \mathbf{f}+q,
$$

where the Newtonian velocity and pressure potentials $\mathbf{N}_{\alpha ; \Omega_{+}} \mathbf{f}$ and $\mathcal{Q}_{\Omega_{+}} \mathbf{f}$ are defined by (3.21). By Remark 3.3,

$$
\begin{aligned}
& \triangle \mathbf{N}_{\alpha ; \Omega_{+}} \mathbf{f}-\alpha \mathbf{N}_{\alpha ; \Omega_{+}} \mathbf{f}-\nabla \mathcal{Q}_{\Omega_{+}} \mathbf{f}=\mathbf{f}, \operatorname{div} \mathbf{N}_{\alpha ; \Omega_{ \pm}} \mathbf{f}=0 \text { in } \Omega_{+} \\
& \left(\mathbf{N}_{\alpha ; \Omega_{+}} \mathbf{f}, \mathcal{Q}_{\Omega_{+}} \mathbf{f}\right) \in B_{p, p^{*}}^{2}\left(\Omega_{+}, \mathbb{R}^{n}\right) \times B_{p, p^{*}}^{1}\left(\Omega_{+}\right), \gamma_{+}\left(\mathbf{N}_{\alpha ; \Omega_{+}} \mathbf{f}\right) \in H_{p ; \nu}^{1}\left(\partial \Omega, \mathbb{R}^{n}\right), \mathbf{t}_{\alpha}^{+}\left(\mathbf{N}_{\alpha ; \Omega_{+}} \mathbf{f}, \mathcal{Q}_{\Omega_{ \pm}} \mathbf{f}\right) \in L_{p}\left(\partial \Omega, \mathbb{R}^{n}\right) .
\end{aligned}
$$

Then problem (5.25) reduces to the one for the corresponding homogeneous Brinkman system,

$$
\left\{\begin{array}{l}
\Delta \mathbf{v}-\alpha \mathbf{v}-\nabla q=\mathbf{0}, \operatorname{div} \mathbf{v}=0 \text { in } \Omega_{+} \\
\gamma_{+} \mathbf{v}=\mathbf{h}_{00}
\end{array}\right.
$$

where $\mathbf{h}_{00}:=\mathbf{h}_{0}-\gamma_{+}\left(\mathbf{N}_{\alpha ; \Omega_{+}} \mathbf{f}\right) \in H_{p ; \nu}^{s}\left(\partial \Omega, \mathbb{R}^{n}\right)$, already discussed in the first paragraph of the proof. Therefore, there exists a solution $(\mathbf{u}, \pi) \in B_{p, p^{*}}^{s+\frac{1}{p}}\left(\Omega_{+}, \mathbb{R}^{n}\right) \times B_{p, p^{*}}^{s+\frac{1}{p}-1}\left(\Omega_{+}\right)$of the Poisson problem (5.25), which satisfies the asserted estimate.

Let us prove the uniqueness of the solution to the Poisson problem (5.25) for $0<s<1$. To do so, we consider a solution $\left(\mathbf{u}^{0}, \pi^{0}\right) \in B_{p, p^{*}}^{s+\frac{1}{p}}\left(\Omega, \mathbb{R}^{3}\right) \times B_{p, p^{*}}^{s+\frac{1}{p}-1}(\Omega)$ of the homogeneous version of the problem (5.25). Let us take the trace of the Green representation formula (3.128) for $\left(\mathbf{u}^{0}, \pi^{0}\right)$. Since $\gamma_{+} \mathbf{u}^{0}=\mathbf{0}$, we obtain the equation

$$
\mathcal{V}_{\alpha}\left(\mathbf{t}_{\alpha}^{+}\left(\mathbf{u}^{0}, \pi^{0}\right)\right)=\mathbf{0} \text { on } \partial \Omega \text {, }
$$

for $\mathbf{t}_{\alpha}^{+}\left(\mathbf{u}^{0}, \pi^{0}\right) \in B_{p, p^{*}}^{s-1}(\partial \Omega)$, which by Corollary 4.7 has a one-dimensional set of solutions, $\mathbf{t}_{\alpha}^{+}\left(\mathbf{u}^{0}, \pi^{0}\right)=c \boldsymbol{\nu}$, where $c \in \mathbb{R}$. Substituting this back into the Green representation formula (3.128) we obtain $\mathbf{u}^{0}=c \mathbf{V}_{\alpha} \boldsymbol{\nu}=\mathbf{0}$ in $\Omega_{+}$(cf. the arguments in the proof of Lemma 4.6), and by the homogeneous Brinkman equation, $\pi^{0}$ is an arbitrary constant. Finally, uniqueness for $0<s<1$ implies also uniqueness for $s=1$. 


\subsection{The Neumann problem for the Brinkman system}

Using an argument similar to the one for the Robin boundary value problem for the Brinkman system in [35], we obtain in this section the well-posedness of the Neumann problem for the linear Brinkman system,

$$
\left\{\begin{array}{l}
\triangle \mathbf{u}-\alpha \mathbf{u}-\nabla \pi=0, \text { in } \Omega_{+}, \\
\operatorname{div} \mathbf{u}=0 \text { in } \Omega_{+}, \\
\mathbf{t}_{\mathrm{nt}}^{+}(\mathbf{u}, \pi)=\mathbf{g}_{0} \text { on } \partial \Omega
\end{array}\right.
$$

in $L_{p}$-based Bessel potential and Besov spaces for some $\varepsilon>0$, and extend the results obtained in the case $p=2$ and for a conormal derivative given by $\frac{\partial \mathbf{u}}{\partial n}:=-\pi \boldsymbol{\nu}+\frac{\partial \mathbf{u}}{\partial \boldsymbol{\nu}}$, in [69, Theorem 5.3] (see also [61, Theorem 5.5.2] in the case $\alpha=0$ ). Note that the Neumann boundary condition in $(5.28)$ is understood in the sense of non-tangential limit almost everywhere on $\partial \Omega$

Theorem 5.5 Let $\Omega_{+} \subset \mathbb{R}^{n}(n \geq 3)$ be a bounded Lipschitz domain with connected boundary $\partial \Omega$. Let $\alpha \in(0, \infty)$. Then there exists $\epsilon>0$, such that for any $p \in \mathcal{R}_{0}(n, \epsilon)$ (see (4.4)), and for any given datum $\mathbf{g}_{0} \in L_{p}\left(\partial \Omega, \mathbb{R}^{n}\right)$, the Neumann problem (5.28) has a unique solution $(\mathbf{u}, \pi)$ such that $M(\mathbf{u}), M(\nabla \mathbf{u}), M(\pi) \in L_{p}(\partial \Omega)$. The solution can be represented by the single layer velocity and pressure potentials

$$
\mathbf{u}=\mathbf{V}_{\alpha}\left(\left(\frac{1}{2} \mathbb{I}+\mathbf{K}_{\alpha}^{*}\right)^{-1} \mathbf{g}_{0}\right), \pi=Q^{s}\left(\left(\frac{1}{2} \mathbb{I}+\mathbf{K}_{\alpha}^{*}\right)^{-1} \mathbf{g}_{0}\right)
$$

Moreover, $(\mathbf{u}, \pi) \in B_{p, p^{*}}^{1+\frac{1}{p}}\left(\Omega_{+}, \mathbb{R}^{n}\right) \times B_{p, p^{*}}^{\frac{1}{p}}\left(\Omega_{+}\right)$, and there exist some constants $C_{M}, C$ and $C^{\prime}$ depending only on $\Omega_{+}, \alpha$, and $p$ such that

$$
\begin{aligned}
& \|M(\nabla \mathbf{u})\|_{L_{p}(\partial \Omega)}+\|M(\mathbf{u})\|_{L_{p}(\partial \Omega)}+\|M(\pi)\|_{L_{p}(\partial \Omega)} \leq C_{M}\left\|\mathbf{g}_{0}\right\|_{L_{p}\left(\partial \Omega, \mathbb{R}^{n}\right)}, \\
& \|\mathbf{u}\|_{B_{p, p^{*}}^{1+\frac{1}{p}}\left(\Omega_{+}, \mathbb{R}^{n}\right)}+\|\pi\|_{B_{p, p^{*}}^{\frac{1}{p}}\left(\Omega_{+}\right)} \leq C\left\|\mathbf{g}_{0}\right\|_{L_{p}\left(\partial \Omega, \mathbb{R}^{n}\right)}, \\
& \left\|\gamma_{+} \mathbf{u}\right\|_{H_{p}^{1}\left(\partial \Omega, \mathbb{R}^{n}\right)}+\left\|\mathbf{t}_{\alpha}^{+}(\mathbf{u}, \pi)\right\|_{L_{p}\left(\partial \Omega, \mathbb{R}^{n}\right)} \leq C^{\prime}\left\|\mathbf{g}_{0}\right\|_{L_{p}\left(\partial \Omega, \mathbb{R}^{n}\right)} .
\end{aligned}
$$

Proof. We use an argument similar to that for [23, Theorem 4.15] (see also [62, Theorem 3.1, Proposition 3.3]). By Lemma 4.2 there exists $\epsilon>0$ such that operator $\frac{1}{2} \mathbb{I}+\mathbf{K}_{\alpha}^{*}: L_{p}\left(\partial \Omega, \mathbb{R}^{n}\right) \rightarrow L_{p}\left(\partial \Omega, \mathbb{R}^{n}\right)$ is an isomorphism for $p \in \mathcal{R}_{0}(n, \epsilon)$. Along with Lemma 3.4, Theorem 3.5 and Lemma 3.6 this implies that representation (5.29) gives a solution of problem (5.28) that belongs to the space $B_{p, p^{*}}^{1+\frac{1}{p}}\left(\Omega_{+}, \mathbb{R}^{n}\right) \times B_{p, p^{*}}^{\frac{1}{p}}\left(\Omega_{+}\right)$and satisfies estimates (5.30)-(5.32).

In order to show the uniqueness assertion, we assume that $\left(\mathbf{u}^{0}, \pi^{0}\right)$ is a solution of the homogeneous version of (5.28) such that $M\left(\mathbf{u}^{0}\right), M\left(\nabla \mathbf{u}^{0}\right), M(\pi)^{0} \in L_{p}(\partial \Omega)$ and satisfies the Neumann condition almost everywhere on $\partial \Omega$ in the sense of non-tangential limit. Then the Green representation formula (3.130) gives,

$$
\mathbf{u}^{0}=\mathbf{V}_{\alpha}\left(\mathbf{t}_{\mathrm{nt}}^{+}\left(\mathbf{u}^{0}, \pi^{0}\right)\right)-\mathbf{W}_{\alpha}\left(\mathbf{u}_{\mathrm{nt}}^{0+}\right)=-\mathbf{W}_{\alpha}\left(\mathbf{u}_{\mathrm{nt}}^{0+}\right) \text { in } \Omega_{+},
$$

which, combined with formulas (3.82), leads to the boundary integral equation

$$
\left(\frac{1}{2} \mathbb{I}+\mathbf{K}_{\alpha}\right) \mathbf{u}_{\mathrm{nt}}^{0+}=\mathbf{0} \text { on } \partial \Omega
$$

Here $\mathbf{u}_{\mathrm{nt}}^{0+} \in H_{p}^{1}\left(\partial \Omega, \mathbb{R}^{n}\right)$ due to Lemma 3.4(i). Then invertibility of operator (4.9) in Lemma 4.2 implies that $\mathbf{u}_{\mathrm{nt}}^{0+}=\mathbf{0}$ on $\partial \Omega$ and thus, by (5.33), $\mathbf{u}^{0}=\mathbf{0}$ in $\Omega_{+}$. Moreover, by the homogeneous Neumann condition satisfied by $\left(\mathbf{u}^{0}, \pi^{0}\right)$, we obtain that $\pi^{0}=0$ in $\Omega_{+}$. This concludes the proof of uniqueness of the solution of the Neumann problem (5.28), and hence the proof of the theorem.

Having in view Theorem 5.5, we are now able to consider the Poisson-Neumann problem for the Brinkman system,

$$
\left\{\begin{array}{l}
\triangle \mathbf{u}-\alpha \mathbf{u}-\nabla \pi=\mathbf{f}, \operatorname{div} \mathbf{u}=0 \text { in } \Omega_{+} \\
\mathbf{t}_{\alpha}^{+}(\mathbf{u}, \pi)=\mathbf{g}_{0} \text { on } \partial \Omega
\end{array}\right.
$$

with the Neumann datum for the canonical conormal derivative $\mathbf{t}_{\alpha}^{+}(\mathbf{u}, \pi)$ (see also [62, Theorem 10.6.4] for $\alpha=0$ ).

Theorem 5.6 Let $\Omega_{+} \subset \mathbb{R}^{n}(n \geq 3)$ be a bounded Lipschitz domain with connected boundary $\partial \Omega$. Let $\alpha \in(0, \infty)$. Then there exists $\varepsilon=\varepsilon(\partial \Omega)>0$ such that for any $p \in \mathcal{R}_{0}(n, \epsilon)\left(c f\right.$. (4.4)), the Neumann problem (5.35) with $\mathbf{f} \in L_{p}\left(\Omega_{+}, \mathbb{R}^{3}\right)$ and $\mathbf{g}_{0} \in L_{p}\left(\partial \Omega, \mathbb{R}^{n}\right)$ has a unique solution $(\mathbf{u}, \pi) \in B_{p, p^{*}}^{1+\frac{1}{p}}\left(\Omega_{+}, \mathbb{R}^{n}\right) \times B_{p, p^{*}}^{\frac{1}{p}}\left(\Omega_{+}\right)$, where $p^{*}=\max \{2, p\}$. In addition, there exists a constant $C=C\left(p, \Omega_{+}\right)>0$ such that

$$
\|\mathbf{u}\|_{B_{p, p^{*}}^{1+\frac{1}{p}}\left(\Omega_{+}, \mathbb{R}^{n}\right)}+\|\pi\|_{B_{p, p^{*}}^{\frac{1}{p}}\left(\Omega_{+}\right)} \leq C\left(\|\mathbf{f}\|_{L_{p}\left(\Omega_{+}, \mathbb{R}^{n}\right)}+\left\|\mathbf{g}_{0}\right\|_{L_{p}\left(\partial \Omega, \mathbb{R}^{n}\right)}\right)
$$




$$
\left\|\gamma_{+} \mathbf{u}\right\|_{H_{p}^{1}\left(\partial \Omega, \mathbb{R}^{n}\right)} \leq C\left(\|\mathbf{f}\|_{L_{p}\left(\Omega_{+}, \mathbb{R}^{n}\right)}+\mathbf{g}_{0} \|_{L_{p}\left(\partial \Omega, \mathbb{R}^{n}\right)}\right) .
$$

Moreover, if $\mathbf{f}=\mathbf{0}$, then $M(\mathbf{u}), M(\nabla \mathbf{u}), M(\pi) \in L_{p}(\partial \Omega)$ and there exists a constant $C_{M}>0$ such that

$$
\|M(\mathbf{u})\|_{L_{p}(\partial \Omega)}+\|M(\nabla \mathbf{u})\|_{L_{p}(\partial \Omega)}+\|M(\pi)\|_{L_{p}(\partial \Omega)} \leq C_{M}\left\|\mathbf{g}_{0}\right\|_{L_{p}\left(\partial \Omega, \mathbb{R}^{n}\right)}
$$

Proof. If $\mathbf{f}=\mathbf{0}$, there exists a solution of problem (5.35) given by the solution of the corresponding problem (5.28) with the non-tangential conormal derivative in the Neumann condition, whose existence is provided by Theorem 5.5 together with the asserted estimate. Here we rely also on the equivalence of the conormal derivatives, $\mathbf{t}_{\alpha}^{+}(\mathbf{u}, \pi)=\mathbf{t}_{\mathrm{nt}}^{+}(\mathbf{u}, \pi)$, due to Theorem 2.13 .

If $\mathbf{f} \neq \mathbf{0}$, we will look for a solution of problem (5.35) in the form

$$
\mathbf{u}=\mathbf{N}_{\alpha ; \Omega_{+}} \mathbf{f}+\mathbf{v}, \pi=\mathcal{Q}_{\Omega_{+}} \mathbf{f}+q,
$$

where the Newtonian velocity and pressure potentials $\mathbf{N}_{\alpha ; \Omega_{+}} \mathbf{f}$ and $\mathcal{Q}_{\Omega_{+}} \mathbf{f}$ are defined by (3.21). According to Remark 3.3, we obtain the relations

$$
\begin{aligned}
& \triangle \mathbf{N}_{\alpha ; \Omega_{+}} \mathbf{f}-\alpha \mathbf{N}_{\alpha ; \Omega_{+}} \mathbf{f}-\nabla \mathcal{Q}_{\Omega_{+}} \mathbf{f}=\mathbf{f}, \operatorname{div} \mathbf{N}_{\alpha ; \Omega_{ \pm}} \mathbf{f}=0 \text { in } \Omega_{+}, \\
& \left(\mathbf{N}_{\alpha ; \Omega_{+}} \mathbf{f}, \mathcal{Q}_{\Omega_{+}} \mathbf{f}\right) \in B_{p, p^{*}}^{2}\left(\Omega_{+}, \mathbb{R}^{n}\right) \times B_{p, p^{*}}^{1}\left(\Omega_{+}\right), \gamma_{+}\left(\mathbf{N}_{\alpha ; \Omega_{+}} \mathbf{f}\right) \in H_{p ; \nu}^{1}\left(\partial \Omega, \mathbb{R}^{n}\right), \mathbf{t}^{+}\left(\mathbf{N}_{\alpha ; \Omega_{+}} \mathbf{f}, \mathcal{Q}_{\Omega_{ \pm}} \mathbf{f}\right) \in L_{p}\left(\partial \Omega, \mathbb{R}^{n}\right) .
\end{aligned}
$$

Then problem (5.35) reduces to the problem for the corresponding homogeneous Brinkman system,

$$
\left\{\begin{array}{l}
\triangle \mathbf{v}-\alpha \mathbf{v}-\nabla q=\mathbf{0}, \operatorname{div} \mathbf{v}=0 \text { in } \Omega_{+} \\
\mathbf{t}_{\alpha}^{+}(\mathbf{u}, \pi)=\mathbf{g}_{00} \text { on } \partial \Omega
\end{array}\right.
$$

where $\mathbf{g}_{00}:=\mathbf{g}_{0}-\mathbf{t}_{\alpha}^{+}\left(\mathbf{N}_{\alpha ; \Omega_{ \pm}} \mathbf{f}_{ \pm}, \mathcal{Q}_{\Omega_{ \pm}} \mathbf{f}_{ \pm}\right) \in L_{p}\left(\partial \Omega, \mathbb{R}^{n}\right)$, already discussed in the first paragraph of the proof. Therefore, there exists a solution $(\mathbf{u}, \pi) \in B_{p, p^{*}}^{1+\frac{1}{p}}\left(\Omega_{+}, \mathbb{R}^{n}\right) \times B_{p, p^{*}}^{\frac{1}{p}}\left(\Omega_{+}\right)$of the Poisson problem (5.35), which satisfies all the asserted estimates.

Let us prove uniqueness of the solution to the Poisson problem (5.35). Indeed, let us consider a solution $\left(\mathbf{u}^{0}, \pi^{0}\right) \in$ $B_{p, p^{*}}^{1+\frac{1}{p}}\left(\Omega, \mathbb{R}^{3}\right) \times B_{p, p^{*}}^{\frac{1}{p}}(\Omega)$ of the homogeneous version of problem (5.35). Let us take the trace of the Green representation formula (3.128) for $\left(\mathbf{u}^{0}, \pi^{0}\right)$, considered for any $s \in(0,1)$. Since $\mathbf{t}_{\alpha}^{+}(\mathbf{u}, \pi)=\mathbf{0}$, we obtain the equation

$$
\gamma_{+} \mathbf{u}^{0}=\frac{1}{2} \gamma_{+} \mathbf{u}^{0}-\mathbf{K}_{\alpha} \gamma_{+} \mathbf{u}^{0} \text { on } \partial \Omega
$$

with the unknown $\gamma_{+} \mathbf{u}^{0} \in B_{p, p^{*}}^{s}\left(\partial \Omega, \mathbb{R}^{n}\right)$, which, by Corollary 4.3, has only the trivial solution. Substituting this back to the Green representation formula (3.128) we obtain $\mathbf{u}^{0}=\mathbf{0}$ in $\Omega_{+}$. Then the Brinkman system implies $\pi^{0}=c \in \mathbb{R}$, and taking again into account that $\mathbf{t}_{\alpha}^{+}(\mathbf{u}, \pi)=\mathbf{0}$, we obtain $\pi^{0}=0$ in $\Omega_{+}$, as asserted.

\section{The mixed Dirichlet-Neumann problem for the Brinkman system}

In this section we show the well-posedness of the mixed Dirichlet-Neumann boundary value problem for the Brinkman system

$$
\left\{\begin{array}{l}
\triangle \mathbf{u}-\alpha \mathbf{u}-\nabla \pi=\mathbf{0}, \operatorname{div} \mathbf{u}=0 \text { in } \Omega_{+}, \\
\mathbf{u}_{\mathrm{nt}}^{+} \mid s_{D}=\mathbf{h}_{0}, \\
\left.\mathbf{t}_{\mathrm{nt}}^{+}(\mathbf{u}, \pi)\right|_{S_{N}}=\mathbf{g}_{0},
\end{array}\right.
$$

on a bounded, creased Lipschitz domain $\Omega_{+} \subset \mathbb{R}^{n}(n \geq 3)$ with connected boundary $\partial \Omega$, which is decomposed into two disjoint admissible patches $S_{D}$ and $S_{N}$ (see Definition 6.3), $\left.\cdot\right|_{S_{D}}$ is the operator of restriction from $H_{p}^{s}\left(\partial \Omega, \mathbb{R}^{n}\right)$ to $H_{p}^{s}\left(S_{D}, \mathbb{R}^{n}\right)$, and $\left.\cdot\right|_{N}$ is defined similarly. We show that for $\mathbf{h}_{0} \in H_{p}^{1}\left(S_{D}, \mathbb{R}^{n}\right)$ and $\mathbf{g}_{0} \in L_{p}\left(S_{N}, \mathbb{R}^{n}\right)$ given and for some range of $p$, there exists a unique solution $(\mathbf{u}, \pi)$ of the mixed problem (6.1), such that $M(\mathbf{u}), M(\nabla \mathbf{u}), M(\pi) \in L_{p}(\partial \Omega)$, and the Dirichlet and Neumann boundary conditions in (6.1) are satisfied in the sense of non-tangential limits at almost all points of $S_{D}$ and $S_{N}$, respectively. Moreover, we will show that $(\mathbf{u}, \pi) \in B_{p, p^{*}}^{1+\frac{1}{p}}\left(\Omega_{+}, \mathbb{R}^{n}\right) \times B_{p, p^{*}}^{\frac{1}{p}}\left(\Omega_{+}\right)$.

We consider also a counterpart mixed problem

$$
\left\{\begin{array}{l}
\Delta \mathbf{u}-\alpha \mathbf{u}-\nabla \pi=\mathbf{0}, \operatorname{div} \mathbf{u}=0 \text { in } \Omega_{+} \\
\left.\gamma_{+} \mathbf{u}\right|_{S_{D}}=\mathbf{h}_{0}, \\
\left.\mathbf{t}_{\alpha}^{+}(\mathbf{u}, \pi)\right|_{S_{N}}=\mathbf{g}_{0},
\end{array}\right.
$$

where, unlike the mixed problem setting (6.1), the trace is considered in the Gagliardo sense and the conormal derivative in the canonical sense. We will show that for $\mathbf{h}_{0} \in H_{p}^{1}\left(S_{D}, \mathbb{R}^{n}\right)$ and $\mathbf{g}_{0} \in L_{p}\left(S_{N}, \mathbb{R}^{n}\right)$ given and for some range of $p$, there exists a unique solution $(\mathbf{u}, \pi) \in B_{p, p^{*}}^{1+\frac{1}{p}}\left(\Omega_{+}, \mathbb{R}^{n}\right) \times B_{p, p^{*}}^{\frac{1}{p}}\left(\Omega_{+}\right)$of problem (6.2). Moreover, we will obtain that $M(\mathbf{u}), M(\nabla \mathbf{u}), M(\pi) \in L_{p}(\partial \Omega)$.

The corresponding mixed problems for the Poisson-Brinkman system, i.e., with non-zero right hand side of the Brinkman system, will be also considered. 


\subsection{Creased Lipschitz domains}

Next, we recall the definition of admissible patch (cf., e.g., [57, Definition 2.1], [9]).

Definition 6.1 Let $\Omega \subset \mathbb{R}^{n}(n \geq 3)$ be a Lipschitz domain. Let $S$ be an open set of $\partial \Omega$, such that for any $x_{0} \in \partial S$ there exists a new orthogonal system obtained from the original one by a rigid motion with $x_{0}$ as the origin and with the property that one can find a cube $Q=Q_{1} \times Q_{2} \times \cdots \times Q_{n} \subset \mathbb{R}^{n}$ centered at 0 and two Lipschitz functions

$$
\begin{cases}\Phi: Q^{\prime}:=Q_{1} \times \ldots \times Q_{n-1} \rightarrow Q_{n}, & \Phi(0)=0 \\ \Psi: Q^{\prime \prime}:=Q_{2} \times \ldots \times Q_{n-1} \rightarrow Q_{1}, & \Psi(0)=0\end{cases}
$$

such that

$$
\begin{aligned}
& S \cap Q=\left\{\left(x^{\prime}, \Phi\left(x^{\prime}\right)\right): x^{\prime} \in Q^{\prime}, \Psi\left(x^{\prime \prime}\right) \leq x_{1}\right\}, \\
& (\partial \Omega \backslash \bar{S}) \cap Q=\left\{\left(x^{\prime}, \Phi\left(x^{\prime}\right)\right): x^{\prime} \in Q^{\prime}, \Psi\left(x^{\prime \prime}\right) \geq x_{1}\right\}, \\
& \partial S \cap Q=\left\{\left(\Psi\left(x^{\prime \prime}\right), x^{\prime \prime}, \Phi\left(\Psi\left(x^{\prime \prime}\right), x^{\prime \prime}\right)\right): x^{\prime \prime} \in Q^{\prime \prime}\right\} .
\end{aligned}
$$

Such a set $S$ is called an admissible patch of $\partial \Omega$.

Definition 6.1 shows that if $S \subset \partial \Omega$ is an admissible patch then $\partial \Omega \backslash \bar{S}$ is also an admissible patch (cf., e.g., [57]). Next, we recall the definition of a creased Lipschitz graph domain (cf. [57, Definition 2.2]).

Definition 6.2 Let $\Omega \subset \mathbb{R}^{n}(n \geq 3)$ be an open, connected set. Suppose that $S_{D}, S_{N} \subset \partial \Omega$ are two non-empty, disjoint admissible patches such that $\overline{S_{D}} \cap \overline{S_{N}}=\partial S_{D}=\partial S_{N}$ and $\overline{S_{D}} \cup \overline{S_{N}}=\partial \Omega$. The set $\Omega$ is a creased Lipschitz graph domain if the following conditions are satisfied:

(a) There exists a Lipschitz function $\phi: \mathbb{R}^{n-1} \rightarrow \mathbb{R}$ such that

$$
\Omega=\left\{\left(x^{\prime}, x_{n}\right) \in \mathbb{R}^{n}: x_{n}>\phi\left(x^{\prime}\right)\right\} .
$$

(b) There exists a Lipschitz function $\psi: \mathbb{R}^{n-2} \rightarrow \mathbb{R}$ such that

$$
\begin{aligned}
& S_{N}=\left\{\left(x_{1}, x^{\prime \prime}, x_{n}\right) \in \mathbb{R}^{n}: x_{1}>\Psi\left(x^{\prime \prime}\right)\right\} \cap \partial \Omega, \\
& S_{D}=\left\{\left(x_{1}, x^{\prime \prime}, x_{n}\right) \in \mathbb{R}^{n}: x_{1}<\Psi\left(x^{\prime \prime}\right)\right\} \cap \partial \Omega .
\end{aligned}
$$

(c) There exist some constants $\delta_{D}, \delta_{N} \geq 0, \delta_{D}+\delta_{N}>0$ with the property that

$$
\frac{\partial \phi}{\partial x_{1}} \geq \delta_{N} \text { a.e. on } S_{N}, \frac{\partial \phi}{\partial x_{1}} \leq-\delta_{D} \text { a.e. on } S_{D}
$$

Let us now refer to a creased bounded Lipschitz domain (cf. [57, Definition 2.3]).

Definition 6.3 Assume that $\Omega \subset \mathbb{R}^{n}$ is a bounded Lipschitz domain with connected boundary $\partial \Omega$, and that $S_{D}, S_{N} \subset \partial \Omega$ are two non-empty, disjoint admissible patches such that $\overline{S_{D}} \cap \overline{S_{N}}=\partial S_{D}=\partial S_{N}$ and $\overline{S_{D}} \cup \overline{S_{N}}=\partial \Omega$. Then $\Omega$ is creased if

(a) There exist $m \in \mathbb{N}, a>0$ and $z_{i} \in \partial \Omega, i=1, \ldots, m$, such that $\partial \Omega \subset \cup_{i=1}^{m} B_{a}\left(z_{i}\right)$, where $B_{a}\left(z_{i}\right)$ is the ball of radius a and center at $z_{i}$

(b) For any point $z_{i}, i=1, \ldots, m$, there exist a coordinate system $\left\{x_{1}, \ldots, x_{n}\right\}$ with origin at $z_{i}$ and a Lipschitz function $\phi_{i}$ from $\mathbb{R}^{n-1}$ to $\mathbb{R}$ such that the set $\Omega_{i}:=\left\{\left(x^{\prime}, x_{n}\right) \in \mathbb{R}^{n}: x_{n}>\phi_{i}\left(x^{\prime}\right)\right\}$, whose boundary $\partial \Omega_{i}$ admits the decomposition $\partial \Omega_{i}=\overline{S_{D_{i}}} \cup \overline{S_{N_{i}}}$, is a creased Lipschitz graph domain in the sense of Definition 6.2, and

$$
\Omega \cap B_{2 a}\left(z_{i}\right)=\Omega_{i} \cap B_{2 a}\left(z_{i}\right), S_{D} \cap B_{2 a}\left(z_{i}\right)=S_{D_{i}} \cap B_{2 a}\left(z_{i}\right), S_{N} \cap B_{2 a}\left(z_{i}\right)=S_{N_{i}} \cap B_{2 a}\left(z_{i}\right) .
$$

The geometric meaning of Definitions 6.2 and 6.3 is that $S_{D}$ and $S_{N}$ are separated by a Lipschitz interface $\left(\overline{S_{D}} \cap \overline{S_{N}}\right.$ is a creased collision manifold for $\mathfrak{D}$ ) and that $S_{D}$ and $S_{N}$ meet at an angle which is strictly less than $\pi$ (cf., e.g., [7, 57]). A main property of a (bounded or graph) creased Lipschitz domain is the existence of a function $\boldsymbol{\varphi} \in C^{\infty}\left(\bar{\Omega}, \mathbb{R}^{n}\right)$ and of a constant $\delta>0$ such that

$$
\boldsymbol{\varphi} \cdot \boldsymbol{\nu}>\delta \text { a.e. on } \boldsymbol{S}_{N}, \boldsymbol{\varphi} \cdot \boldsymbol{\nu}<-\delta \text { a.e. on } \boldsymbol{S}_{D}
$$

i.e., the scalar product $\boldsymbol{\varphi} \cdot \boldsymbol{\nu}$, between $\boldsymbol{\varphi}$ and the unit normal $\boldsymbol{\nu}$, changes the sign when moving from $S_{D}$ to $S_{N}$ (cf., e.g., [8, (1.122)], [9, (2.2)]). For such a domain, Brown [7] showed that the mixed problem for the Laplace equation has a unique solution whose gradient belongs to $L_{2}(\partial \mathfrak{D})$ when the Dirichlet datum belongs to $H_{2}^{1}\left(S_{D}\right)$ and the Neumann datum to $L_{2}\left(S_{N}\right)$. For the same class of domains, well-posedness of the mixed problem for the Laplace equation in a range of $L_{p}-$ based spaces has been obtained in [57]. 


\subsection{Mixed Dirichlet-Neumann problem for the Brinkman system with boundary data in $L_{2}$-based spaces}

Mitrea and Mitrea in [57] have proved sharp well-posedness results for the Poisson problem for the Laplace operator with mixed boundary conditions of Dirichlet and Neumann type on bounded creased Lipschitz domains in $\mathbb{R}^{n}(n \geq 3)$, whose boundaries satisfy a geometric condition, and with data in Sobolev and Besov spaces. Brown et al. in [9, Theorem 1.1] have obtained the well-posedness result for the mixed Dirichlet-Neumann problem for the Stokes system with boundary data in $L_{2}$-based spaces on creased Lipschitz domains in $\mathbb{R}^{n}(n \geq 3)$, by reducing such a boundary value problem to the analysis of a boundary integral equation (see also the references therein). Well-posedness of the mixed Dirichlet-Robin problem for the Brinkman system in a creased Lipschitz domain with boundary data in $L_{2}$-based spaces has been recently proved in [35, Theorem 6.1]. Using the main ideas of that proof, we show in this section well-posedness of the mixed Dirichlet-Neumann boundary value problem for the Brinkman system in $L_{2}$-based Bessel potential spaces defined on a bounded, creased Lipschitz domain $\Omega_{+}$.

Theorem 6.4 Assume that $\Omega_{+} \subset \mathbb{R}^{n}(n \geq 3)$ is a bounded, creased Lipschitz domain with connected boundary $\partial \Omega$, which is decomposed into two disjoint admissible patches $S_{D}$ and $S_{N}$. Then the mixed problem (6.1) with given data $\left(\mathbf{h}_{0}, \mathbf{g}_{0}\right) \in H_{2}^{1}\left(S_{D}, \mathbb{R}^{n}\right) \times L_{2}\left(S_{N}, \mathbb{R}^{n}\right)$ has a unique solution $(\mathbf{u}, \pi)$ such that $M(\mathbf{u}), M(\nabla \mathbf{u}), M(\pi) \in L_{2}(\partial \Omega)$. Moreover, $(\mathbf{u}, \pi) \in$ $H_{2}^{\frac{3}{2}}\left(\Omega_{+}, \mathbb{R}^{n}\right) \times H_{2}^{\frac{1}{2}}\left(\Omega_{+}\right)$, and there exist some constants $C_{M}$ and $C$ depending only on $S_{D}, S_{N}$ and $\alpha$ such that

$$
\begin{aligned}
& \|M(\nabla \mathbf{u})\|_{L_{2}(\partial \Omega)}+\|M(\mathbf{u})\|_{L_{2}(\partial \Omega)}+\|M(\pi)\|_{L_{2}(\partial \Omega)} \leq C_{M}\left(\left\|\mathbf{h}_{0}\right\|_{H_{2}^{1}\left(S_{D}, \mathbb{R}^{n}\right)}+\left\|\mathbf{g}_{0}\right\|_{L_{2}\left(S_{N}, \mathbb{R}^{n}\right)}\right), \\
& \|\mathbf{u}\|_{H_{2}^{\frac{3}{2}}\left(\Omega_{+}, \mathbb{R}^{n}\right)}+\|\pi\|_{H_{2}^{\frac{1}{2}}\left(\Omega_{+}\right)} \leq C\left(\left\|\mathbf{h}_{0}\right\|_{H_{2}^{1}\left(S_{D}, \mathbb{R}^{n}\right)}+\left\|\mathbf{g}_{0}\right\|_{L_{2}\left(S_{N}, \mathbb{R}^{n}\right)}\right) .
\end{aligned}
$$

Proof. First, we note that if a couple $(\mathbf{u}, \pi)$ satisfies the Brinkman system (6.1) and the conditions $M(\mathbf{u}), M(\nabla \mathbf{u}), M(\pi) \in$ $L_{2}(\partial \Omega)$, then, taking into account that $B_{2,2}^{\frac{3}{2}}\left(\Omega_{+}, \mathbb{R}^{n}\right)=H_{2}^{\frac{3}{2}}\left(\Omega_{+}, \mathbb{R}^{n}\right), B_{2,2}^{\frac{1}{2}}\left(\Omega_{+}\right)=H_{2}^{\frac{1}{2}}\left(\Omega_{+}\right)$, Theorem 5.2(ii) implies that $(\mathbf{u}, \pi) \in \mathfrak{H}_{2, \text { div }}^{\frac{3}{2}, t}\left(\Omega, \mathcal{L}_{\alpha}\right)$ for any $t \geq-\frac{1}{2}$, while $\gamma_{+} \mathbf{u}=\mathbf{u}_{\mathrm{nt}}^{+}$and $\mathbf{t}_{\alpha}^{+}(\mathbf{u}, \pi)=\mathbf{t}_{\mathrm{nt}}^{+}(\mathbf{u}, \pi)$ by Theorems 2.5 and 2.13 .

Let us show that the mixed boundary value problem (6.1) has at most one $L_{2}$-solution. Indeed, if a couple $\left(\mathbf{u}^{(0)}, \pi^{(0)}\right)$ satisfies the homogeneous problem associated to (6.1), and moreover $\left(\mathbf{u}^{(0)}, \pi^{(0)}\right) \in \mathfrak{H}_{2, \text { div }}^{\frac{3}{2}, 0}\left(\Omega, \mathcal{L}_{\alpha}\right)$, then by the first Green identity (2.28), we obtain the equality

$$
\left\langle\mathbf{t}_{\alpha}^{+}\left(\mathbf{u}^{(0)}, \pi^{(0)}\right), \gamma_{+} \mathbf{u}^{(0)}\right\rangle_{\partial \Omega}=2\left\langle\mathbb{E}\left(\mathbf{u}^{(0)}\right), \mathbb{E}\left(\mathbf{u}^{(0)}\right)\right\rangle_{\Omega_{+}}+\alpha\left\langle\mathbf{u}^{(0)}, \mathbf{u}^{(0)}\right\rangle_{\Omega_{+}}
$$

where the left-hand side vanishes, due to the homogeneous boundary conditions satisfied by $\gamma_{+} \mathbf{u}^{(0)}=\mathbf{u}_{\mathrm{nt}}^{(0)+}$ and $\mathbf{t}_{\alpha}^{+}\left(\mathbf{u}^{(0)}, \pi^{(0)}\right)=$ $\mathbf{t}_{\mathrm{nt}}^{+}\left(\mathbf{u}^{(0)}, \pi^{(0)}\right)$ on $S_{D}$ and $S_{N}$, respectively. Then by (6.12) we immediately obtain that $\mathbf{u}^{(0)}=\mathbf{0}$ and $\pi^{(0)}=0$ in $\Omega_{+}$.

Next, we consider the operator

$$
\mathcal{S}_{\alpha}: L_{2}\left(\partial \Omega, \mathbb{R}^{n}\right) \rightarrow H_{2}^{1}\left(S_{D}, \mathbb{R}^{n}\right) \times L_{2}\left(S_{N}, \mathbb{R}^{n}\right), \mathcal{S}_{\alpha} \Psi:=\left(\left.\left.\left(\mathcal{V}_{\alpha} \Psi\right)\right|_{S_{D}}\left(\left(\frac{1}{2} \mathbb{I}+\mathbf{K}_{\alpha}^{*}\right) \Psi\right)\right|_{S_{N}}\right)
$$

(cf. [35, (6.6)-(6.8)]), and show that this is an isomorphism, which will yield the well-posedness of the mixed problem (6.1). To this end, we note that $\mathcal{S}_{\alpha}$ can be written as $\mathcal{S}_{\alpha}=\mathcal{S}_{0}+\mathcal{S}_{\alpha ; 0}$, where

$$
\begin{aligned}
& \mathcal{S}_{0}: L_{2}\left(\partial \Omega, \mathbb{R}^{n}\right) \rightarrow H_{2}^{1}\left(S_{D}, \mathbb{R}^{n}\right) \times L_{2}\left(S_{N}, \mathbb{R}^{n}\right), \mathcal{S}_{0} \Psi:=\left(\left.\left(\mathcal{V}_{0} \Psi\right)\right|_{S_{D}},\left.\left(\left(\frac{1}{2} \mathbb{I}+\mathbf{K}_{0}^{*}\right) \Psi\right)\right|_{S_{N}}\right), \\
& \mathcal{S}_{\alpha ; 0}: L_{2}\left(\partial \Omega, \mathbb{R}^{n}\right) \rightarrow H_{2}^{1}\left(S_{D}, \mathbb{R}^{n}\right) \times L_{2}\left(S_{N}, \mathbb{R}^{n}\right), \mathcal{S}_{\alpha ; 0} \Psi:=\left(\left.\left(\mathcal{V}_{\alpha ; 0} \Psi\right)\right|_{S_{D}},\left.\left(\mathbf{K}_{\alpha ; 0}^{*} \Psi\right)\right|_{S_{N}}\right) .
\end{aligned}
$$

Here $\mathcal{V}_{\alpha ; 0}: L_{2}\left(\partial \Omega, \mathbb{R}^{n}\right) \rightarrow H_{2}^{1}\left(\partial \Omega, \mathbb{R}^{n}\right)$ and $\mathbf{K}_{\alpha ; 0}^{*}: L_{2}\left(\partial \Omega, \mathbb{R}^{n}\right) \rightarrow L_{2}\left(\partial \Omega, \mathbb{R}^{n}\right)$ are the complementary layer potential operators defined as

$$
\mathcal{V}_{\alpha ; 0} \Psi:=\mathcal{V}_{\alpha} \Psi-\mathcal{V}_{0} \Psi \text { and } \mathbf{K}_{\alpha ; 0}^{*} \Psi:=\mathbf{K}_{\alpha}^{*} \Psi-\mathbf{K}_{0}^{*} \Psi
$$

The operator $\mathcal{S}_{0}$ defined in (6.14) is an isomorphism and this property is equivalent with the well-posedness result of the mixed Dirichlet-Neumann problem for the Stokes system on a creased Lipschitz domain with Dirichlet and Neumann boundary data in $L_{2}$-based spaces (cf. the proof of [9, Theorem 6.3]), when the BVP solution is looked for in the form of the Stokes single layer potential. In addition, the continuity of the restriction operators from $H_{2}^{1}\left(\partial \Omega, \mathbb{R}^{n}\right)$ to $H_{2}^{1}\left(S_{D}, \mathbb{R}^{n}\right)$ and from $L_{2}\left(\partial \Omega, \mathbb{R}^{n}\right)$ to $L_{2}\left(S_{N}, \mathbb{R}^{n}\right)$, respectively, as well as the compactness of the complementary operators in (6.16) (cf. [33, Theorem 3.4]) imply that the operator $\mathcal{S}_{\alpha ; 0}$ in (6.15) is compact as well. Therefore, the operator $\mathcal{S}_{\alpha}$ in (6.13) is Fredholm with index zero. This operator is also injective. Indeed, if $\Psi^{(0)} \in L_{2}\left(\partial \Omega, \mathbb{R}^{n}\right)$ satisfies the equation $\mathcal{S}_{\alpha} \psi^{(0)}=0$ then the single layer velocity and pressure potentials $\mathbf{u}^{(0)}:=\mathbf{V}_{\alpha} \Psi^{(0)}$ and $\pi^{(0)}:=\mathcal{Q}^{s} \Psi^{(0)}$ will determine a solution of the homogeneous mixed problem associated to (6.1), such that $\left(\mathbf{u}^{(0)}, \pi^{(0)}\right) \in H_{2}^{\frac{3}{2}}\left(\Omega_{+}, \mathbb{R}^{n}\right) \times H_{2}^{\frac{1}{2}}\left(\Omega_{+}\right)$and $M\left(\mathbf{u}^{(0)}\right), M\left(\nabla \mathbf{u}^{(0)}\right), M\left(\pi^{(0)}\right) \in L_{2}(\partial \Omega)$. Then $\mathbf{u}^{(0)}=\mathbf{0}$ and $\pi^{(0)}=0$ in $\Omega_{+}$, as shown above. Consequently, $\mathbf{t}_{\mathrm{nt}}^{+}\left(\mathbf{u}^{(0)}, \boldsymbol{\pi}^{(0)}\right)=\mathbf{0}$ a.e. on $\partial \Omega$, which, in view of (3.83), can be written as

$$
\left(\frac{1}{2} \mathbb{I}+\mathbf{K}_{\alpha}^{*}\right) \Psi^{(0)}=\mathbf{0}
$$


Moreover, the invertibility of the operator $\frac{1}{2} \mathbb{I}+\mathbf{K}_{\alpha}^{*}: L_{2}\left(\partial \Omega, \mathbb{R}^{n}\right) \rightarrow L_{2}\left(\partial \Omega, \mathbb{R}^{n}\right)$ (see Lemma 4.2$)$ shows that $\Psi^{(0)}=\mathbf{0}$. Consequently, operator (6.13) is an isomorphism, as asserted. Then the fields

$$
\mathbf{u}=\mathbf{V}_{\alpha}\left(\mathcal{S}_{\alpha}^{-1}\left(\mathbf{h}_{0}, \mathbf{g}_{0}\right)\right), \quad \pi=\mathcal{Q}^{s}\left(\mathcal{S}_{\alpha}^{-1}\left(\mathbf{h}_{0}, \mathbf{g}_{0}\right)\right)
$$

determine the unique solution of the mixed Dirichlet-Neumann problem (6.1). According to Lemma 3.4, Theorem 3.5 and (6.17), the solution belongs to the space $H_{2}^{\frac{3}{2}}\left(\Omega_{+}, \mathbb{R}^{n}\right) \times H_{2}^{\frac{1}{2}}\left(\Omega_{+}\right)$and satisfies the estimate (6.10) with some constant $C_{M}>0$ depending on $S_{D}, S_{N}$ and $\alpha$, as well as estimate (6.11) with the constant $C=\left(\left\|\mathbf{V}_{\alpha}\right\|+\left\|Q^{s}\right\|\right)\left\|\mathcal{S}_{\alpha}^{-1}\right\|$.

\subsection{Mixed Dirichlet-Neumann problem for the Brinkman system with data in $L_{p}$-spaces}

Next, we extend the results established in Theorem 6.4 , to $L_{p}$-based spaces with $p$ in some neighborhood of 2 , for the mixed Dirichlet-Neumann problem for the Brinkman system (6.1), with the boundary data $\left(\mathbf{h}_{0}, \mathbf{g}_{0}\right) \in H_{p}^{1}\left(S_{D}, \mathbb{R}^{n}\right) \times L_{p}\left(S_{N}, \mathbb{R}^{n}\right)$. We will obtain the well-posedness result in the space $B_{p, p^{*}}^{1+\frac{1}{p}}\left(\Omega_{+}, \mathbb{R}^{n}\right) \times B_{p, p^{*}}^{\frac{1}{p}}\left(\Omega_{+}\right)$, where $p^{*}=\max \{2, p\}$.

We further need the space

$$
\widetilde{H}_{p}^{0}\left(S_{0}, \mathbb{R}^{n}\right):=\left\{\boldsymbol{\Phi} \in L_{p}\left(\partial \Omega, \mathbb{R}^{n}\right): \operatorname{supp} \boldsymbol{\Phi} \subseteq \overline{S_{0}}\right\}, \quad S_{0} \subset \partial \Omega
$$

\section{- The Neumann-to-Dirichlet operator for the Brinkman system}

As in the work [57], devoted to the mixed Dirichlet-Neumann problem for the Laplace equation in a creased Lipschitz domain, we consider the Neumann-to-Dirichlet operator $\Upsilon_{\mathrm{nt} ; \alpha}$, which associates to $\mathbf{g} \in L_{p}\left(\partial \Omega, \mathbb{R}^{n}\right)$, the restriction of the non-tangential trace $\mathbf{u}_{\mathrm{nt}}^{+}$to the patch $S_{D}$, where $(\mathbf{u}, \pi)$ is the unique $L_{p}$-solution of the Neumann problem (5.28) for the Brinkman system with the non-tangential conormal derivative $\mathbf{g}$. Thus, $(\mathbf{u}, \pi)$ satisfies the Neumann condition almost everywhere on $\partial \Omega$ in the sense of non-tangential limit, as well as the conditions $M(\mathbf{u}), M(\nabla \mathbf{u}), M(\pi) \in L_{p}(\partial \Omega)$, and

$$
\Upsilon_{\mathrm{nt} ; \alpha} \mathbf{g}=\mathbf{u}_{\mathrm{nt}}^{+} \mid s_{D} \text {. }
$$

Similarly, we consider the Neumann-to-Dirichlet operator $\Upsilon_{\alpha}$, which associates to $\mathbf{g} \in L_{p}\left(\partial \Omega\right.$, $\left.\mathbb{R}^{n}\right)$, the restriction of the trace $\gamma_{+} \mathbf{u}$ to the patch $S_{D}$, where $(\mathbf{u}, \pi)$ is the unique solution of the Neumann problem (5.35) for the Brinkman system with $\mathbf{f}=\mathbf{0}$ and the canonical conormal derivative $\mathbf{g}$, i.e.,

$$
\Upsilon_{\alpha} \mathbf{g}=\left.\gamma_{+} \mathbf{u}\right|_{S_{D}}
$$

A way to extend the well-posedness result in Theorem 6.4 to $L_{p}$-based spaces is to show the invertibility of the Neumann-toDirichlet operator $\Upsilon_{n t ; \alpha}$ on such spaces. An intermediary step to obtain this property is given by the following result.

Lemma 6.5 Let $\Omega_{+} \subset \mathbb{R}^{n}(n \geq 3)$ be a bounded, creased Lipschitz domain with connected boundary $\partial \Omega$ which is decomposed into two disjoint admissible patches $S_{D}$ and $S_{N}$. Let $\alpha \in(0, \infty)$. Then there exists $\varepsilon=\varepsilon(\partial \Omega)>0$ such that for any $p \in \mathcal{R}_{0}(n, \varepsilon)$ the following properties hold.

(i) The operators $\Upsilon_{\mathrm{nt} ; \alpha}$ and $\Upsilon_{\alpha}$ coincide and are given by

$$
\Upsilon_{\mathrm{nt} ; \alpha}=\Upsilon_{\alpha}=\left.\left(\mathcal{V}_{\alpha} \circ\left(\frac{1}{2} \mathbb{I}+\mathbf{K}_{\alpha}^{*}\right)^{-1}\right)\right|_{S_{D}} .
$$

(ii) The mixed Dirichlet-Neumann problem (6.1) with given data $\left(\mathbf{h}_{0}, \mathbf{g}_{0}\right) \in H_{p}^{1}\left(S_{D}, \mathbb{R}^{n}\right) \times L_{p}\left(S_{N}, \mathbb{R}^{n}\right)$ has a unique solution $(\mathbf{u}, \pi)$, such that $M(\mathbf{u}), M(\nabla \mathbf{u}), M(\pi) \in L_{p}(\partial \Omega)$, if and only if the operator

$$
\Upsilon_{\mathrm{nt} ; \alpha}: \widetilde{H}_{p}^{0}\left(S_{D}, \mathbb{R}^{n}\right) \rightarrow H_{p}^{1}\left(S_{D}, \mathbb{R}^{n}\right)
$$

is an isomorphism.

(iii) The mixed Dirichlet-Neumann problem (6.2) with given data $\left(\mathbf{h}_{0}, \mathbf{g}_{0}\right) \in H_{p}^{1}\left(S_{D}, \mathbb{R}^{n}\right) \times L_{p}\left(S_{N}, \mathbb{R}^{n}\right)$ has a unique solution $(\mathbf{u}, \pi) \in B_{p, p^{*}}^{1+\frac{1}{p}}\left(\Omega_{+}, \mathbb{R}^{n}\right) \times B_{p, p^{*}}^{\frac{1}{p}}\left(\Omega_{+}\right)$if and only if the operator

$$
\Upsilon_{\alpha}: \widetilde{H}_{p}^{0}\left(S_{D}, \mathbb{R}^{n}\right) \rightarrow H_{p}^{1}\left(S_{D}, \mathbb{R}^{n}\right)
$$

is an isomorphism.

Moreover, when the solution $(\mathbf{u}, \pi)$ in item (ii) or (iii) exists, then it belongs to the space $B_{p, p^{*}}^{1+\frac{1}{p}}\left(\Omega_{+}, \mathbb{R}^{n}\right) \times B_{p, p^{*}}^{\frac{1}{p}}\left(\Omega_{+}\right)$ and there exist some constants $C_{M} \equiv C_{M}\left(\alpha, p, S_{D}, S_{N}\right), C \equiv C\left(\alpha, p, S_{D}, S_{N}\right)$ and $C^{\prime} \equiv C^{\prime}\left(\alpha, p, S_{D}, S_{N}\right)$ such that

$$
\begin{aligned}
& \|M(\nabla \mathbf{u})\|_{L_{p}(\partial \Omega)}+\|M(\mathbf{u})\|_{L_{p}(\partial \Omega)}+\|M(\pi)\|_{L_{p}(\partial \Omega)} \leq C_{M}\left(\left\|\mathbf{h}_{0}\right\|_{H_{p}^{1}\left(S_{D}, \mathbb{R}^{n}\right)}+\left\|\mathbf{g}_{0}\right\|_{L_{p}\left(S_{N}, \mathbb{R}^{n}\right)}\right), \\
& \|\mathbf{u}\|_{B_{p, p^{*}}^{1+\frac{1}{p}}\left(\Omega_{+}, \mathbb{R}^{n}\right)}+\|\pi\|_{B_{p, p^{*}}^{\frac{1}{p}}\left(\Omega_{+}\right)} \leq C\left(\left\|\mathbf{h}_{0}\right\|_{H_{p}^{1}\left(S_{D}, \mathbb{R}^{n}\right)}+\left\|\mathbf{g}_{0}\right\|_{L_{p}\left(S_{N}, \mathbb{R}^{n}\right)}\right), \quad p^{*}=\max \{2, p\}, \\
& \left\|\boldsymbol{\gamma}_{+} \mathbf{u}\right\|_{H_{p}^{1}\left(\partial \Omega, \mathbb{R}^{n}\right)}+\left\|\mathbf{t}_{\alpha}^{+}(\mathbf{u}, \pi)\right\|_{\left.L_{p}\left(\partial \Omega, \mathbb{R}^{n}\right)\right)} \leq C^{\prime}\left(\left\|\mathbf{h}_{0}\right\|_{H_{p}^{1}\left(S_{D}, \mathbb{R}^{n}\right)}+\left\|\mathbf{g}_{0}\right\|_{L_{p}\left(S_{N}, \mathbb{R}^{n}\right)}\right) .
\end{aligned}
$$


Proof. (i) By Theorem 5.5, there exists $\varepsilon=\varepsilon(\partial \Omega)>0$ such that for any $p \in \mathcal{R}_{0}(n, \varepsilon)$ the Neumann problem (5.28) has a unique solution, and it can be expressed in form (5.29). Then due to Theorem 3.5 and Lemma 3.6 we deduce that the operator (6.19) has the expression (6.21) and is continuous, due to the continuity of both operators in the right-hand side of (6.21).

(ii) First, we assume that problem (6.1) is well-posed and show the invertibility of operator (6.22).

In order to prove the injectivity property of this operator, we consider a function $\mathbf{g}^{0} \in \widetilde{H}_{p}^{0}\left(S_{D}, \mathbb{R}^{n}\right)$, such that $\Upsilon_{\mathrm{nt} ; \alpha} \mathbf{g}^{0}=\mathbf{0}$. Denoting by $\left(\mathbf{u}^{0}, \pi^{0}\right)$ the unique $L_{p^{-}}$solution of the Neumann problem (5.28) for the homogeneous Brinkman system with boundary datum $\mathbf{g}^{0} \in \widetilde{H}_{p}^{0}\left(S_{D}, \mathbb{R}^{n}\right)$ on $\partial \Omega$, in view of (6.19), we have

$$
\mathbf{u}_{\mathrm{nt}}^{+} \mid s_{D}=\Upsilon_{\mathrm{nt} ; \alpha} \mathbf{g}^{0}=\mathbf{0}
$$

and

$$
\left\{\begin{array}{l}
\triangle \mathbf{u}^{0}-\alpha \mathbf{u}^{0}-\nabla \pi^{0}=\mathbf{0}, \operatorname{div} \mathbf{u}^{0}=0 \text { in } \Omega_{+}, \\
\mathbf{t}_{\mathrm{nt}}^{+}\left(\mathbf{u}^{0}, \pi^{0}\right)=\mathbf{g}^{0} \text { on } \partial \Omega .
\end{array}\right.
$$

In addition, $\left(\mathbf{u}^{0}, \pi^{0}\right)$ satisfies the conditions $M\left(\mathbf{u}^{0}\right), M\left(\nabla \mathbf{u}^{0}\right), M\left(\pi^{0}\right) \in L_{p}(\partial \Omega)$, and the Neumann condition holds almost everywhere on $\partial \Omega$ in the sense of non-tangential limit.

According to relation (6.27) and the inclusion $\mathbf{g}^{0} \in \widetilde{H}_{p}^{0}\left(S_{D}, \mathbb{R}^{n}\right)$, we have

$$
\mathbf{u}_{\mathrm{nt}}^{0+} \mid S_{D}=\mathbf{0} \text { on } S_{D},\left.\mathbf{t}_{\mathrm{nt}}^{+}\left(\mathbf{u}^{0}, \pi^{0}\right)\right|_{S_{N}}=\mathbf{0} \text { on } S_{N},
$$

and hence by the assumed well-posedness of the mixed Dirichlet-Neumann problem (6.1), we deduce that $\mathbf{u}^{0}=\mathbf{0}$ and $\pi^{0}=0$ in $\Omega_{+}$. Thus, $\mathbf{g}^{0}=\mathbf{t}_{\mathrm{nt}}^{+}\left(\mathbf{u}^{0}, \pi^{0}\right)=\mathbf{0}$ on $\partial \Omega$, which implies that the operator $\Upsilon_{\alpha}$ is injective.

We show that the operator $\Upsilon_{\mathrm{nt} ; \alpha}$ is also surjective. Due to the assumed well posedness of the mixed Dirichlet-Neumann problem (6.1), for any Dirichlet datum $\mathbf{h}_{0} \in H_{p}^{1}\left(S_{D}, \mathbb{R}^{n}\right)$ on $S_{D}$ and the Neumann datum $\mathbf{g}_{0} \equiv \mathbf{0}$ on $S_{N}$, there exists a unique

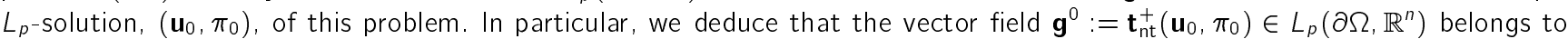
$\widetilde{H}_{p}^{0}\left(S_{D}, \mathbb{R}^{n}\right)$, due to definition (6.18). In addition, the uniqueness result in Theorem 5.5 shows that $\left(\mathbf{u}_{0}, \pi_{0}\right)$ is the unique solution of the Neumann problem for the Brinkman system in $\Omega_{+}$with the Neumann datum $\mathbf{g}^{0} \in \widetilde{H}_{p}^{0}\left(S_{D}, \mathbb{R}^{n}\right) \subset L_{p}\left(\partial \Omega, \mathbb{R}^{n}\right)$. Then by definition (6.19) of the operator $\Upsilon_{\mathrm{nt} ; \alpha}$, we obtain that $\Upsilon_{\mathrm{nt} ; \alpha} \mathbf{g}_{0}=\mathbf{u}_{0, \mathrm{nt}}^{+} \mid s_{D}=\mathbf{h}_{0}$. Consequently, for a given $\mathbf{h}_{0} \in H_{p}^{1}\left(S_{D}\right.$, $\left.\mathbb{R}^{n}\right)$ there exists $\mathbf{g}_{0} \in \widetilde{H}_{p}^{0}\left(S_{D}, \mathbb{R}^{n}\right)$ such that $\Upsilon_{n t ; \alpha} \mathbf{g}_{0}=\mathbf{h}_{0}$. This shows that the operator $\Upsilon_{n t ; \alpha}$ is surjective, and thus, it is an isomorphism, as asserted.

Next, we show the converse result, i.e., that the invertibility of the operator $\Upsilon_{\mathrm{nt} ; \alpha}$ implies the well-posedness of the mixed Dirichlet-Neumann problem (6.1). Let us first show uniqueness of the solution to problem (6.1). To this end, we assume that $\left(\mathbf{u}^{(0)}, \pi^{(0)}\right)$ is an $L_{p}$-solution of the homogeneous version of $(6.1)$. Hence, $\mathbf{g}^{(0)}:=\mathbf{t}_{\mathrm{nt}}^{+}\left(\mathbf{u}^{(0)}, \pi^{(0)}\right) \in \widetilde{H}_{p}^{0}\left(S_{D}, \mathbb{R}^{n}\right)$ since $\left.\mathbf{t}_{\mathrm{nt}}^{+}\left(\mathbf{u}^{(0)}, \boldsymbol{\pi}^{(0)}\right)\right|_{S_{N}}=\mathbf{0}$, implying that $\left(\mathbf{u}^{(0)}, \boldsymbol{\pi}^{(0)}\right)$ is (by Theorem 5.5) the unique solution of the Neumann problem for the Brinkman system with Neumann datum $\mathbf{g}^{(0)}$ on $\partial \Omega$. Then by (6.19), $\Upsilon_{\mathrm{nt} ; \alpha} \mathbf{g}^{(0)}=\mathbf{u}_{\mathrm{nt}}^{(0)+} \mid s_{D}=\mathbf{0}$, and injectivity of $\Upsilon_{\mathrm{nt} ; \alpha}$ implies that $\mathbf{g}^{(0)}=\mathbf{0}$. Hence $\mathbf{t}_{\mathrm{nt}}^{+}\left(\mathbf{u}^{(0)}, \boldsymbol{\pi}^{(0)}\right)=\mathbf{0}$ on $\partial \Omega$ and Theorem 5.5 implies that $\mathbf{u}^{0}=\mathbf{0}, \pi^{0}=0$ in $\Omega_{+}$. This concludes the proof of uniqueness of the solution to the mixed problem (6.1).

To show existence of an $L_{p^{-}}$solution to the mixed problem (6.1), let us consider such a problem with arbitrary boundary data $\left(\mathbf{h}_{0}, \mathbf{g}_{0}\right) \in H_{p}^{1}\left(S_{D}, \mathbb{R}^{n}\right) \times L_{p}\left(S_{N}, \mathbb{R}^{n}\right)$. Also let $\mathbf{G} \in \widetilde{H}_{p}^{0}\left(S_{N}, \mathbb{R}^{n}\right)$ be such that

$$
\left.\mathbf{G}\right|_{S_{N}}=\mathbf{g}_{0} \text {. }
$$

Then by Theorem 5.5 there exists a unique $L_{p}$-solution $(\mathbf{v}, q)$ of the Neumann problem (5.28) with the Neumann datum $\mathbf{G}$, such that there exist the non-tangential limits of $\mathbf{u}, \nabla \mathbf{u}, \pi$ at almost all points of $\partial \Omega, M(\mathbf{v}), M(\nabla \mathbf{v}), M(q) \in L_{2}(\partial \Omega)$, and satisfies the Neumann boundary condition in the sense of non-tangential limit at almost all points of $\partial \Omega$. Note that $\mathbf{v}$ can be expressed in terms of a single-layer potential with a density in the space $L_{p}\left(\partial \Omega, \mathbb{R}^{n}\right)$, and hence $\mathbf{v}_{\mathrm{nt}}^{+} \in H_{p}^{1}\left(\partial \Omega\right.$, $\left.\mathbb{R}^{n}\right)$ (see Lemma 3.6).

On the other hand, the invertibility of the operator $\Upsilon_{n t ; \alpha}: \widetilde{H}_{p}^{0}\left(S_{D}, \mathbb{R}^{n}\right) \rightarrow H_{p}^{1}\left(S_{D}, \mathbb{R}^{n}\right)$ assures that the equation

$$
\Upsilon_{\mathrm{nt} ; \alpha} \mathbf{g}^{0}=\left(\mathbf{h}_{0}-\left.\mathbf{v}_{\mathrm{nt}}^{+}\right|_{S_{D}}\right) \in H_{p}^{1}\left(S_{D}, \mathbb{R}^{n}\right)
$$

has a unique solution $\mathbf{g}^{0} \in \widetilde{H}_{p}^{0}\left(S_{D}, \mathbb{R}^{n}\right) \subset L_{p}\left(\partial \Omega, \mathbb{R}^{n}\right)$. Next, let $\left(\mathbf{u}^{0}, \pi^{0}\right)$ be the unique $L_{p}$-solution of the Neumann problem (5.28) with the Neumann datum $\mathbf{g}^{0}$. Also let

$$
(\mathbf{u}, \pi):=\left(\mathbf{v}+\mathbf{u}^{0}, q+\pi^{0}\right)
$$

Then we obtain the relations

$$
\begin{aligned}
\mathbf{u}_{\mathrm{nt}}^{+} \mid s_{D} & =\mathbf{v}_{\mathrm{nt}}^{+}\left|s_{D}+\mathbf{u}_{\mathrm{nt}}^{0+}\right| s_{D}=\left(\mathbf{h}_{0}-\Upsilon_{\mathrm{nt} ; \alpha} \mathbf{g}^{0}\right)+\Upsilon_{\mathrm{nt} ; \alpha} \mathbf{g}^{0}=\mathbf{h}_{0}, \\
\mathbf{t}_{\mathrm{nt}}^{+}(\mathbf{u}, \pi) \mid s_{N} & =\mathbf{t}_{\mathrm{nt}}^{+}(\mathbf{v}, q)\left|s_{N}+\mathbf{t}_{\mathrm{nt}}^{+}\left(\mathbf{u}^{0}, \pi^{0}\right)\right| s_{N}=\left.\mathbf{G}\right|_{s_{N}}+\mathbf{g}^{0} \mid s_{N}=\mathbf{g}_{0},
\end{aligned}
$$

where the last equality follows from (6.30) and the inclusion $\mathbf{g}^{0} \in \widetilde{H}_{p}^{0}\left(S_{D}, \mathbb{R}^{n}\right)$. Moreover, the estimates (6.24) and (6.25) corresponding to item (ii) are due to (6.32) and the mapping properties of the pairs $(\mathbf{v}, q)$ and $\left(\mathbf{u}^{0}, \pi^{0}\right)$ given by Theorem 5.5 . Consequently, the mixed Dirichlet-Neumann problem (6.1) is well-posed and estimates (6.24)-(6.26) hold true. 
The proof for item (iii) of the lemma and estimates (6.24)-(6.26) follow from similar arguments as those for item (ii), by refering to Theorems 5.4 and 5.6 instead of Theorems 5.1 and 5.5.

By combining Theorem 6.4 and Lemma 6.5, we are now able to obtain the well-posedness results for the mixed DirichletNeumann problem (6.1) with boundary data in $L_{p}$-based Bessel potential spaces and with $p$ in a neighborhood of 2 , which is the main result of this section. Recall that $p^{*}=\max \{2, p\}$.

Theorem 6.6 Assume that $\Omega_{+} \subset \mathbb{R}^{n}(n \geq 3)$ is a bounded, creased Lipschitz domain with connected boundary $\partial \Omega$ which is decomposed into two disjoint admissible patches $S_{D}$ and $S_{N}$. Then there exists a number $\varepsilon>0$ such that for any $p \in(2-\varepsilon, 2+\varepsilon)$ and for all given data $\left(\mathbf{h}_{0}, \mathbf{g}_{0}\right) \in H_{p}^{1}\left(S_{D}, \mathbb{R}^{n}\right) \times L_{p}\left(S_{N}, \mathbb{R}^{n}\right)$ the following properties hold.

(i) The mixed Dirichlet-Neumann problem for the Brinkman system (6.1) has a unique solution (u, $\pi$ ), such that $M(\mathbf{u}), M(\nabla \mathbf{u}), M(\pi) \in L_{p}(\partial \Omega)$. Moreover, $(\mathbf{u}, \pi) \in B_{p, p^{*}}^{1+\frac{1}{p}}\left(\Omega_{+}, \mathbb{R}^{n}\right) \times B_{p, p^{*}}^{\frac{1}{p}}\left(\Omega_{+}\right)$, and there exist some constants $C_{M} \equiv$ $C_{M}\left(\alpha, p, S_{D}, S_{N}\right)>0, C \equiv C\left(\alpha, p, S_{D}, S_{N}\right)>0$ and $C^{\prime} \equiv C^{\prime}\left(\alpha, p, S_{D}, S_{N}\right)>0$ such that

$$
\begin{aligned}
& \|M(\nabla \mathbf{u})\|_{L_{p}(\partial \Omega)}+\|M(\mathbf{u})\|_{L_{p}(\partial \Omega)}+\|M(\pi)\|_{L_{p}(\partial \Omega)} \leq C_{M}\left(\left\|\mathbf{h}_{0}\right\|_{H_{p}^{1}\left(S_{D}, \mathbb{R}^{n}\right)}+\left\|\mathbf{g}_{0}\right\|_{L_{p}\left(S_{N}, \mathbb{R}^{n}\right)}\right), \\
& \|\mathbf{u}\|_{B_{p, p^{*}}^{1+\frac{1}{p}}\left(\Omega_{+}, \mathbb{R}^{n}\right)}+\|\pi\|_{B_{p, p^{*}}^{\frac{1}{p}}\left(\Omega_{+}\right)} \leq C\left(\left\|\mathbf{h}_{0}\right\|_{H_{p}^{1}\left(S_{D}, \mathbb{R}^{n}\right)}+\left\|\mathbf{g}_{0}\right\|_{L_{p}\left(S_{N}, \mathbb{R}^{n}\right)}\right), \\
& \left\|\boldsymbol{\gamma}_{+} \mathbf{u}\right\|_{H_{p}^{1}\left(\partial \Omega, \mathbb{R}^{n}\right)}+\left\|\mathbf{t}_{\alpha}^{+}(\mathbf{u}, \pi)\right\|_{\left.L_{p}\left(\partial \Omega, \mathbb{R}^{n}\right)\right)} \leq C^{\prime}\left(\left\|\mathbf{h}_{0}\right\|_{H_{p}^{1}\left(S_{D}, \mathbb{R}^{n}\right)}+\left\|\mathbf{g}_{0}\right\|_{L_{p}\left(S_{N}, \mathbb{R}^{n}\right)}\right) .
\end{aligned}
$$

(ii) The mixed Dirichlet-Neumann problem for the Brinkman system (6.2) has a unique solution $(\mathbf{u}, \pi) \in B_{p, p^{*}}^{1+\frac{1}{p}}\left(\Omega_{+}, \mathbb{R}^{n}\right) \times$ $B_{p, p^{*}}^{\frac{1}{p}}\left(\Omega_{+}\right)$. Moreover, the solution satisfies estimates (6.35)-(6.37).

Proof. (i) By Theorem 6.4 the mixed Dirichlet-Neumann problem (6.1) is well-posed for $p=2$. Then by Lemma 6.5 (ii) and Theorem 5.5 for $p=2$, the operator $\Upsilon_{\mathrm{nt} ; \alpha}: \widetilde{H}_{2}^{0}\left(S_{D}, \mathbb{R}^{n}\right) \rightarrow H_{2}^{1}\left(S_{D}, \mathbb{R}^{n}\right)$ is an isomorphism. Moreover, by Lemma A.1, the sets $\left\{\widetilde{H}_{p}^{0}\left(S_{D}, \mathbb{R}^{n}\right)\right\}_{p \geq 1}$ and $\left\{H_{p}^{1}\left(S_{D}, \mathbb{R}^{n}\right)\right\}_{p \geq 1}$ are complex interpolation scales. Then by the stability of the invertibility property given in Lemma B.2, there exists a number $\varepsilon_{1}>0$, such that the operator $\Upsilon_{\mathrm{nt} ; \alpha}: \widetilde{H}_{p}^{0}\left(S_{D}, \mathbb{R}^{n}\right) \rightarrow H_{p}^{1}\left(S_{D}, \mathbb{R}^{n}\right)$ is an isomorphism as well, for any $p \in\left(2-\varepsilon_{1}, 2+\varepsilon_{1}\right)$. Finally, by choosing the parameter $\varepsilon:=\min \left\{\epsilon, \varepsilon_{1}\right\}>0$, where $\epsilon$ is the parameter in Theorem 5.5 , and by using again Lemma 6.5 (ii), we deduce the well-posedness result of the mixed Dirichlet-Neumann problem (6.1) and estimates (6.35)-(6.37), whenever $p \in(2-\varepsilon, 2+\varepsilon)$.

(ii) Let $\varepsilon$ be as in the proof of item (i). Let $p \in(2-\varepsilon, 2+\varepsilon)$. Then Lemma 6.5 (i) implies that $\Upsilon_{\alpha}=\Upsilon_{\text {nt; } \alpha \text {, and hence }}$ $\Upsilon_{\alpha}: \widetilde{H}_{p}^{0}\left(S_{D}, \mathbb{R}^{n}\right) \rightarrow H_{p}^{1}\left(S_{D}, \mathbb{R}^{n}\right)$ is an isomorphism, and by Lemma 6.5 (ii) the mixed Dirichlet-Neumann problem (6.2) is well posed and estimates (6.35)-(6.37) hold.

Remark 6.7 Under the conditions of Theorem 6.6, the solution $(\mathbf{u}, \pi)$ of the mixed Dirichlet-Neumann problem (6.1) can be expressed by the single layer velocity and pressure potentials

$$
\mathbf{u}=\mathbf{V}_{\alpha}\left(\mathcal{S}_{\alpha}^{-1}\left(\mathbf{h}_{0}, \mathbf{g}_{0}\right)\right), \quad \pi=\mathcal{Q}_{\partial \Omega}^{s}\left(\mathcal{S}_{\alpha}^{-1}\left(\mathbf{h}_{0}, \mathbf{g}_{0}\right)\right),
$$

where the operator

$$
\mathcal{S}_{\alpha}: L_{p}\left(\partial \Omega, \mathbb{R}^{n}\right) \rightarrow H_{p}^{1}\left(S_{D}, \mathbb{R}^{n}\right) \times L_{p}\left(S_{N}, \mathbb{R}^{n}\right), \mathcal{S}_{\alpha} \Psi:=\left(\left.\left.\left(\mathcal{V}_{\alpha} \Psi\right)\right|_{S_{D}}\left(\left(\frac{1}{2} \mathbb{I}+\mathbf{K}_{\alpha}^{*}\right) \Psi\right)\right|_{S_{N}}\right)
$$

is an isomorphism. Indeed, as shown in the proof of Theorem 6.4 , the operator $\mathcal{S}_{\alpha}: L_{2}\left(\partial \Omega, \mathbb{R}^{n}\right) \rightarrow H_{2}^{1}\left(S_{D}, \mathbb{R}^{n}\right) \times L_{2}\left(S_{N}, \mathbb{R}^{n}\right)$ is an isomorphism, and then, by using again Lemma A.1 and Lemma B.2, we can extend the isomorphism property of the operator (6.39) to $L_{p}$-spaces, with $p$ in a neighborhood of 2 , which can be chosen to coincide with that in Theorem 6.6.

\subsection{Poisson problem of mixed Dirichlet-Neumann type for the Brinkman system with data in $L_{p}$-based spaces}

Having in view Theorem 6.6, we are now able to consider the well-posedness of the following Poisson problem of mixed DirichletNeumann type for the Brinkman system in a creased Lipschitz domain $\Omega_{+}$, with data in some $L_{p}$-based spaces,

$$
\left\{\begin{array}{l}
\triangle \mathbf{u}-\alpha \mathbf{u}-\nabla \pi=\mathbf{f} \in L_{p}\left(\Omega_{+}, \mathbb{R}^{3}\right), \operatorname{div} \mathbf{u}=0 \text { in } \Omega_{+} \\
\left.\gamma_{+} \mathbf{u}\right|_{S_{D}}=\mathbf{h}_{0} \in H_{p}^{1}\left(S_{D}, \mathbb{R}^{n}\right) \\
\left.\mathbf{t}_{\alpha}^{+}(\mathbf{u}, \pi)\right|_{S_{N}}=\mathbf{g}_{0} \in L_{p}\left(S_{N}, \mathbb{R}^{n}\right) .
\end{array}\right.
$$

Remark 6.8 (i) By a solution of the Poisson problem of mixed Dirichlet-Neumann type (6.40) we mean a pair (u, $\pi$ ) $\epsilon$ $B_{p, p^{*}}^{1+\frac{1}{p}}\left(\Omega_{+}, \mathbb{R}^{n}\right) \times B_{p, p^{*}}^{\frac{1}{p}}\left(\Omega_{+}\right)$, where $p^{*}=\max \{2, p\}$, which satisfies the non-homogeneous Brinkman system in $\Omega_{+}$, the Dirichlet 
boundary condition on $S_{D}$ in the Gagliardo trace sense, and the Neumann boundary condition on $S_{N}$ in the canonical sense described in Definition 2.10.

(ii) If a pair $(\mathbf{u}, \pi) \in B_{p, p^{*}}^{1+\frac{1}{p}}\left(\Omega_{+}, \mathbb{R}^{n}\right) \times B_{p, p^{*}}^{\frac{1}{p}}\left(\Omega_{+}\right), p \in(1, \infty)$, solves the non-homogeneous Brinkman system in the first line of (6.40) with $\mathbf{f} \in L_{p}\left(\partial \Omega, \mathbb{R}^{n}\right)$, then $(\mathbf{u}, \pi) \in \mathfrak{B}_{p, p^{*} \text {,div }}^{1+\frac{1}{p}, 0}\left(\Omega_{+} ; \mathcal{L}_{\alpha}\right)$ by Definition 2.6. Hence, by Lemma 2.4, Definition 2.10, Lemma 2.11 and the embeddings $B_{p, p^{*}}^{1+\frac{1}{p}}\left(\Omega_{+}, \mathbb{R}^{n}\right) \hookrightarrow B_{p, p^{*}}^{s+\frac{1}{p}}\left(\Omega_{+}, \mathbb{R}^{n}\right), \mathfrak{B}_{p, p^{*}, \text { div }}^{1+\frac{1}{2}, 0}\left(\Omega_{+} ; \mathcal{L}_{\alpha}\right) \hookrightarrow \mathfrak{B}_{p, p^{*} \text {,div }}^{s+\frac{1}{p^{\prime}}}\left(\Omega_{+} ; \mathcal{L}_{\alpha}\right)$, for any $0<s<1$, the trace $\gamma_{+} \mathbf{u}$ and canonical conormal derivative $\mathbf{t}_{\alpha}^{+}(\mathbf{u}, \pi)$ are well defined and belong to $B_{p, p^{*}}^{s}\left(\partial \Omega, \mathbb{R}^{n}\right)$ and $B_{p, p^{*}}^{s-1}\left(\partial \Omega, \mathbb{R}^{n}\right)$, respectively. Thus, the boundary conditions in (6.40) are well defined as well. In what follows, we show that the sharper inclusions, $\gamma_{+} \mathbf{u} \in H_{p}^{1}\left(\partial \Omega, \mathbb{R}^{n}\right)$ and $\mathbf{t}_{\alpha}^{+}(\mathbf{u}, \pi) \in L_{p}\left(\partial \Omega, \mathbb{R}^{n}\right)$, hold if the spaces of the given boundary data in the boundary conditions are those mentioned in (6.40).

Theorem 6.9 Assume that $\Omega_{+} \subset \mathbb{R}^{n}(n \geq 3)$ is a bounded, creased Lipschitz domain with connected boundary $\partial \Omega$, and that $\partial \Omega$ is decomposed into two disjoint admissible patches $S_{D}$ and $S_{N}$. Then there exists a number $\varepsilon>0$ such that for any $p \in(2-\varepsilon, 2+\varepsilon)$ and for all given data $\left(\mathbf{f}, \mathbf{h}_{0}, \mathbf{g}_{0}\right) \in L_{p}\left(\Omega_{+}, \mathbb{R}^{n}\right) \times H_{p}^{1}\left(S_{D}, \mathbb{R}^{n}\right) \times L_{p}\left(S_{N}, \mathbb{R}^{n}\right)$ the Poisson problem of mixed Dirichlet-Neumann type (6.40) has a solution $(\mathbf{u}, \pi) \in B_{p, p^{*}}^{1+\frac{1}{p}}\left(\Omega_{+}, \mathbb{R}^{n}\right) \times B_{p, p^{*}}^{\frac{1}{p}}\left(\Omega_{+}\right)$that can be represented in the form

$$
\mathbf{u}=\mathbf{N}_{\alpha ; \Omega_{+}} \mathbf{f}+\mathbf{V}_{\alpha}\left(\mathcal{S}_{\alpha}^{-1}\left(\mathbf{h}_{00}, \mathbf{g}_{00}\right)\right), \pi=\mathcal{Q}_{\Omega_{+}} \mathbf{f}+\mathcal{Q}_{\partial \Omega}^{s}\left(\mathcal{S}_{\alpha}^{-1}\left(\mathbf{h}_{00}, \mathbf{g}_{00}\right)\right)
$$

where $\mathcal{S}_{\alpha}: L_{p}\left(\partial \Omega, \mathbb{R}^{n}\right) \rightarrow H_{p}^{1}\left(S_{D}, \mathbb{R}^{n}\right) \times L_{p}\left(S_{N}, \mathbb{R}^{n}\right)$ is the isomorphism defined in (6.39), and

$$
\mathbf{h}_{00}:=\mathbf{h}_{0}-\left.\gamma_{+}\left(\mathbf{N}_{\alpha ; \Omega_{+}} \mathbf{f}\right)\right|_{s_{D}} \in H_{p}^{1}\left(S_{D}, \mathbb{R}^{n}\right), \quad \mathbf{g}_{00}:=\mathbf{g}_{0}-\left.\mathbf{t}_{\alpha}^{+}\left(\mathbf{N}_{\alpha ; \Omega_{+}} \mathbf{f}, \mathcal{Q}_{\alpha ; \Omega_{+}} \mathbf{f}\right)\right|_{s_{N}} \in L_{p}\left(S_{N}, \mathbb{R}^{n}\right)
$$

Moreover, the solution $(\mathbf{u}, \pi)$ is unique in the space $B_{p, p^{*}}^{1+\frac{1}{p}}\left(\Omega_{+}, \mathbb{R}^{n}\right) \times B_{p, p^{*}}^{\frac{1}{p}}\left(\Omega_{+}\right)$, and there exist some constants $C \equiv C\left(\alpha, p, S_{D}, S_{N}\right)>0$ and $C^{\prime} \equiv C^{\prime}\left(\alpha, p, S_{D}, S_{N}\right)>0$ such that the following inequalities hold

$$
\begin{aligned}
&\|\mathbf{u}\|_{B_{p, p^{*}}^{1+\frac{1}{p}}\left(\Omega_{+}, \mathbb{R}^{n}\right)}+\|\pi\|_{B_{p, p^{*}}^{\frac{1}{p}}\left(\Omega_{+}\right)} \leq C\left(\mathbf{f}\left\|_{L_{p}\left(\Omega_{+}, \mathbb{R}^{n}\right)}+\right\| \mathbf{h}_{0}\left\|_{H_{p}^{1}\left(S_{D}, \mathbb{R}^{n}\right)}+\right\| \mathbf{g}_{0} \|_{L_{p}\left(S_{N}, \mathbb{R}^{n}\right)}\right), \\
&\left\|\gamma_{+} \mathbf{u}\right\|_{H_{p}^{1}\left(\partial \Omega, \mathbb{R}^{n}\right)}+\left\|\mathbf{t}_{\alpha}^{+}(\mathbf{u}, \pi)\right\|_{L_{p}\left(\partial \Omega, \mathbb{R}^{n}\right)} \leq C^{\prime}\left(\mathbf{f}\left\|_{L_{p}\left(\Omega_{+}, \mathbb{R}^{n}\right)}+\right\| \mathbf{h}_{0}\left\|_{H_{p}^{1}\left(S_{D}, \mathbb{R}^{n}\right)}+\right\| \mathbf{g}_{0} \|_{L_{p}\left(S_{N}, \mathbb{R}^{n}\right)}\right) .
\end{aligned}
$$

In addition, there exists a linear continuous operator

$$
\mathcal{A}_{p}: L_{p}\left(\Omega_{+}, \mathbb{R}^{n}\right) \times H_{p}^{1}\left(S_{D}, \mathbb{R}^{n}\right) \times L_{p}\left(S_{N}, \mathbb{R}^{n}\right) \rightarrow B_{p, p^{*}}^{1+\frac{1}{p}}\left(\Omega_{+}, \mathbb{R}^{n}\right) \times B_{p, p^{*}}^{\frac{1}{p}}\left(\Omega_{+}\right)
$$

delivering this solution, i.e., $\mathcal{A}_{p}\left(\mathbf{f}, \mathbf{h}_{0}, \mathbf{g}_{0}\right)=(\mathbf{u}, \pi)$.

Proof. Let $\varepsilon>0$ as in Theorem 6.6, and let $p \in(2-\varepsilon, 2+\varepsilon)$. We will look for a solution of problem (6.40) in the form

$$
\mathbf{u}=\mathbf{N}_{\alpha ; \Omega_{+}} \mathbf{f}+\mathbf{v}, \pi=\mathcal{Q}_{\Omega_{+}} \mathbf{f}+q,
$$

where the Newtonian velocity and pressure potentials $\mathbf{N}_{\alpha ; \Omega_{+}} \mathbf{f}$ and $\mathcal{Q}_{\Omega_{+}} \mathbf{f}$ are defined by (3.21). By properties (3.23)-(3.26), Corollary 3.2 and Remark 3.3, we obtain that

$$
\begin{aligned}
& \triangle \mathbf{N}_{\alpha ; \Omega_{+}} \mathbf{f}-\alpha \mathbf{N}_{\alpha ; \Omega_{+}} \mathbf{f}-\nabla \mathcal{Q}_{\Omega_{+}} \mathbf{f}=\mathbf{f}, \operatorname{div} \mathbf{N}_{\alpha ; \Omega_{ \pm}} \mathbf{f}=0 \text { in } \Omega_{+}, \\
& \left(\mathbf{N}_{\alpha ; \Omega_{+}} \mathbf{f}, \mathcal{Q}_{\Omega_{+}} \mathbf{f}\right) \in H_{p}^{2}\left(\Omega_{+}, \mathbb{R}^{n}\right) \times H_{p}^{1}\left(\Omega_{+}\right) \hookrightarrow B_{p, p^{*}}^{1+\frac{1}{p}}\left(\Omega_{+}, \mathbb{R}^{n}\right) \times B_{p, p^{*}}^{\frac{1}{p}}\left(\Omega_{+}\right), \\
& \gamma_{+} \mathbf{N}_{\alpha ; \Omega_{+}} \mathbf{f} \in H_{p}^{1}\left(\partial \Omega, \mathbb{R}^{n}\right), \quad \mathbf{t}_{\alpha}^{+}\left(\mathbf{N}_{\alpha ; \Omega_{+}} \mathbf{f}, \mathcal{Q}_{\Omega_{+}} \mathbf{f}\right) \in L_{p}\left(\partial \Omega, \mathbb{R}^{n}\right),
\end{aligned}
$$

where $\gamma_{+}$is the Gagliardo trace operator from $H_{p}^{2}\left(\Omega_{+}, \mathbb{R}^{n}\right)$ to $H_{p}^{1}\left(\partial \Omega, \mathbb{R}^{n}\right)$. Then the mixed Poisson problem (6.40) reduces to the mixed problem for the corresponding homogeneous system,

$$
\left\{\begin{array}{l}
\Delta \mathbf{v}-\alpha \mathbf{v}-\nabla q=\mathbf{0}, \operatorname{div} \mathbf{v}=0 \text { in } \Omega_{+}, \\
\left.\gamma_{+} \mathbf{v}\right|_{S_{D}}=\mathbf{h}_{00} \in H_{p}^{1}\left(S_{D}, \mathbb{R}^{n}\right), \\
\left.\mathbf{t}_{\alpha}^{+}(\mathbf{v}, q)\right|_{S_{N}}=\mathbf{g}_{00} \in L_{p}\left(S_{N}, \mathbb{R}^{n}\right),
\end{array}\right.
$$

where $\mathbf{h}_{00} \in H_{p}^{1}\left(S_{D}, \mathbb{R}^{n}\right)$ and $\mathbf{g}_{00} \in L_{p}\left(S_{N}, \mathbb{R}^{n}\right)$ are given by (6.42), and these inclusions follow from (6.47).

By Theorem 6.6(ii), there exists a unique solution $(\mathbf{v}, q) \in B_{p, p^{*}}^{1+\frac{1}{p}}\left(\Omega_{+}, \mathbb{R}^{n}\right) \times B_{p, p^{*}}^{\frac{1}{p}}\left(\Omega_{+}\right)$of problem (6.49), and it satisfies the following estimates

$$
\|\mathbf{v}\|_{B_{p, p^{*}}^{1+\frac{1}{p}}\left(\Omega_{+}, \mathbb{R}^{n}\right)}+\|q\|_{B_{p, p^{*}}^{\frac{1}{p}}\left(\Omega_{+}\right)} \leq C\left(\left\|\mathbf{h}_{00}\right\|_{H_{p}^{1}\left(S_{D}, \mathbb{R}^{n}\right)}+\left\|\mathbf{g}_{00}\right\|_{L_{p}\left(S_{N}, \mathbb{R}^{n}\right)}\right)
$$




$$
\left\|\gamma_{+} \mathbf{v}\right\|_{H_{p}^{1}\left(\partial \Omega, \mathbb{R}^{n}\right)}+\left\|\mathbf{t}_{\alpha}^{+}(\mathbf{v}, q)\right\|_{\left.L_{p}\left(\partial \Omega, \mathbb{R}^{n}\right)\right)} \leq c^{\prime}\left(\left\|\mathbf{h}_{00}\right\|_{H_{p}^{1}\left(S_{D}, \mathbb{R}^{n}\right)}+\left\|\mathbf{g}_{00}\right\|_{L_{p}\left(S_{N}, \mathbb{R}^{n}\right)}\right)
$$

with some constants $c \equiv c\left(\alpha, p, S_{D}, S_{N}\right)>0$ and $c^{\prime} \equiv c^{\prime}\left(\alpha, p, S_{D}, S_{N}\right)>0$.

According to Lemma 3.6 the single layer velocity and pressure potentials

$$
\mathbf{v}=\mathbf{V}_{\alpha}\left(\mathcal{S}_{\alpha}^{-1}\left(\mathbf{h}_{00}, \mathbf{g}_{00}\right)\right), \quad q=\mathcal{Q}_{\partial \Omega}^{s}\left(\mathcal{S}_{\alpha}^{-1}\left(\mathbf{h}_{00}, \mathbf{g}_{00}\right)\right),
$$

where $\mathcal{S}_{\alpha}: L_{p}\left(\partial \Omega, \mathbb{R}^{n}\right) \rightarrow H_{p}^{1}\left(S_{D}, \mathbb{R}^{n}\right) \times L_{p}\left(S_{N}, \mathbb{R}^{n}\right)$ is the isomorphism defined by (6.39), determine the unique solution of problem (6.49). Moreover, in view of Theorem 3.5 (i) and Lemma 3.6, the pair ( $\mathbf{v}, q)$ given by (6.52) belongs indeed to the space $B_{p, p^{*}}^{1+\frac{1}{p}}\left(\Omega_{+}, \mathbb{R}^{n}\right) \times B_{p, p^{*}}^{\frac{1}{p}}\left(\Omega_{+}\right)$,

Therefore, there exists a solution $(\mathbf{u}, \pi) \in B_{p, p^{*}}^{1+\frac{1}{p}}\left(\Omega_{+}, \mathbb{R}^{n}\right) \times B_{p, p^{*}}^{\frac{1}{p}}\left(\Omega_{+}\right)$of the mixed Poisson problem (6.40), which is given by representation (6.41) and satisfies estimates (6.43) and (6.44). The uniquness result of such a solution follows from Theorem 6.6 (ii). Moreover, linearity and continuity of the Newtonian potential operators (3.25), (3.26) and estimate (6.50) imply the continuity of the operator $\mathcal{A}_{p}$ delivering such a solution.

\section{Mixed Dirichlet-Neumann problem for the semilinear Darcy-Forchheimer-Brinkman system in Besov spaces}

Next we consider the mixed Dirichlet-Neumann problem for the semilinear Darcy-Forchheimer-Brinkman system

$$
\triangle \mathbf{u}-\alpha \mathbf{u}-\beta|\mathbf{u}| \mathbf{u}-\nabla \pi=\mathbf{f}, \quad \operatorname{div} \mathbf{u}=0 \text { in } \Omega_{+} .
$$

Such a nonlinear system describes flows in porous media saturated with viscous incompressible fluids (see, e.g., [65, p.17]), and the constants $\alpha, \beta>0$ are related by the physical properties of such a porous medium, as they describe the viscosity and the convection of the fluid flow.

Due to some embedding results that play a main role in our arguments, we will restrict our analysis in this section to the cases $n=3$

A numerical study of a mixed Dirichlet-Neumann problem for system (7.1) in the particular case of a two-dimensional square cavity driven by a moving wall has been obtained in [26]. Well-posedness and numerical results for an extended nonlinear system, called the Darcy-Forchheimer-Brinkman system, where both semilinear and nonlinear terms $|\mathbf{u}| \mathbf{u}$ and $(\mathbf{u} \cdot \nabla) \mathbf{u}$ are involved, have been obtained in [25], and boundary value problems of Robin type for the Darcy-Forchheimer-Brinkman system with data in $L_{2}$-based Bessel potential (Sobolev) spaces have been studied in [34, 35].

In what follows, we extend an existence and uniqueness result obtained in [35, Theorem 7.1] for the mixed problem (7.3) with the given data in $L_{2}$-based Sobolev spaces, to the case of $L_{p}$-based Bessel potential spaces, i.e., when the given boundary data $\left(\mathbf{h}_{0}, \mathbf{g}_{0}\right)$ belong to the space $H_{p}^{1}\left(S_{D}, \mathbb{R}^{n}\right) \times L_{p}\left(S_{N}, \mathbb{R}^{n}\right)$, with $p \in(2-\varepsilon, 2+\varepsilon)$, and the parameter $\varepsilon>0$ as in Theorem 6.9 . In addition, the given data should be sufficiently small in a sense that will be specified below.

Theorem 7.1 Assume that $\Omega_{+} \subset \mathbb{R}^{3}$ is a bounded creased Lipschitz domain with connected boundary $\partial \Omega$, and that $\partial \Omega$ is decomposed into two disjoint admissible patches $S_{D}$ and $S_{N}$. Let $\alpha, \beta>0$ be given constants. Then there exists a number $\varepsilon>0$ such that for any $p \in(2-\varepsilon, 2+\varepsilon)$ and $p^{*}=\max \{2, p\}$, there exist two constants $\zeta_{p} \equiv \zeta_{p}\left(\Omega_{+}, \alpha, \beta, p\right)>0$ and $\eta_{p} \equiv \eta_{p}\left(\Omega_{+}, \alpha, \beta, p\right)>0$ with the property that for all given data $\left(\mathbf{f}, \mathbf{h}_{0}, \mathbf{g}_{0}\right) \in L_{p}\left(\Omega_{+}, \mathbb{R}^{3}\right) \times H_{p}^{1}\left(S_{D}, \mathbb{R}^{3}\right) \times L_{p}\left(S_{N}, \mathbb{R}^{3}\right)$ satisfying the condition

$$
\left\|\mathbf{h}_{0}\right\|_{H_{p}^{1}\left(S_{D}, \mathbb{R}^{3}\right)}+\left\|\mathbf{g}_{0}\right\|_{L_{p}\left(S_{N}, \mathbb{R}^{3}\right)}+\|\mathbf{f}\|_{L_{p}\left(\Omega_{+}, \mathbb{R}^{3}\right)} \leq \zeta_{p},
$$

the mixed Dirichlet-Neumann problem for the semilinear Darcy-Forchheimer-Brinkman system

$$
\left\{\begin{array}{l}
\Delta \mathbf{u}-\alpha \mathbf{u}-\beta|\mathbf{u}| \mathbf{u}-\nabla \pi=\mathbf{f}, \operatorname{div} \mathbf{u}=0 \text { in } \Omega_{+}, \\
\left.\gamma_{+} \mathbf{u}\right|_{S_{D}}=\mathbf{h}_{0} \text { on } S_{D} \\
\left.\mathbf{t}_{\alpha}^{+}(\mathbf{u}, \pi)\right|_{S_{N}}=\mathbf{g}_{0} \text { on } S_{N}
\end{array}\right.
$$

has a unique solution $(\mathbf{u}, \pi) \in B_{p, p^{*}}^{1+\frac{1}{p}}\left(\Omega_{+}, \mathbb{R}^{n}\right) \times B_{p, p^{*}}^{\frac{1}{p}}\left(\Omega_{+}\right)$, which satisfies the inequality

$$
\|\mathbf{u}\|_{B_{p, p^{*}}^{1+\frac{1}{p}}\left(\Omega_{+}, \mathbb{R}^{n}\right)} \leq \eta_{p} .
$$

Moreover, $\gamma_{+} \mathbf{u} \in H_{p}^{1}\left(\partial \Omega, \mathbb{R}^{n}\right), \mathbf{t}_{\alpha}^{+}(\mathbf{u}, \pi) \in L_{p}\left(\partial \Omega, \mathbb{R}^{n}\right)$ and the solution depends continuously on the given data, which means that there exists some constants $C_{*} \equiv C_{*}\left(\Omega_{+}, \alpha, \beta, p\right)>0$ and $C_{*}^{\prime} \equiv C_{*}\left(\Omega_{+}, \alpha, \beta, p\right)>0$ such that

$$
\begin{array}{r}
\|\mathbf{u}\|_{B_{p, p^{*}}^{1+\frac{1}{p}}\left(\Omega_{+}, \mathbb{R}^{n}\right)}+\|\pi\|_{B_{p, p^{*}}^{\frac{1}{p}}\left(\Omega_{+}\right)} \leq C_{*}\left(\|\mathbf{f}\|_{L_{p}\left(\Omega_{+}, \mathbb{R}^{n}\right)}+\left\|\mathbf{h}_{0}\right\|_{H_{p}^{1}\left(S_{D}, \mathbb{R}^{n}\right)}+\left\|\mathbf{g}_{0}\right\|_{L_{p}\left(S_{N}, \mathbb{R}^{n}\right)}\right), \\
\left\|\gamma_{+} \mathbf{u}\right\|_{H_{p}^{1}\left(\partial \Omega, \mathbb{R}^{n}\right)}+\left\|\mathbf{t}_{\alpha}^{+}(\mathbf{u}, \pi)\right\|_{\left.L_{p}\left(\partial \Omega, \mathbb{R}^{n}\right)\right)} \leq C_{*}^{\prime}\left(\|\mathbf{f}\|_{L_{p}\left(\Omega_{+}, \mathbb{R}^{n}\right)}+\left\|\mathbf{h}_{0}\right\|_{H_{p}^{1}\left(S_{D}, \mathbb{R}^{n}\right)}+\left\|\mathbf{g}_{0}\right\|_{L_{p}\left(S_{N}, \mathbb{R}^{n}\right)}\right) .
\end{array}
$$


Proof. We use the arguments similar to those in the proof of [32, Theorem 5.2] devoted to transmission problems with Lipschitz interface in $\mathbb{R}^{n}$ for the Stokes and Darcy-Forchheimer-Brinkman systems in $L_{2}$-based Sobolev spaces.

According to (A.7) and the second formula in (A.8), for $n \leq 5$ and $p>3 / 2$, we obtain the following continuous embeddings,

$$
B_{p, p^{*}}^{1+\frac{1}{p}}\left(\Omega_{+}, \mathbb{R}^{n}\right) \hookrightarrow B_{2 p, \min \left\{2 p,(2 p)^{\prime}\right\}}^{0}\left(\Omega_{+}, \mathbb{R}^{n}\right) \hookrightarrow H_{2 p}^{0}\left(\Omega_{+}, \mathbb{R}^{n}\right)=L_{2 p}\left(\Omega_{+}, \mathbb{R}^{n}\right) .
$$

Now, by (7.7) and the Hölder inequality we obtain the estimates

$$
\||\mathbf{v}| \mathbf{w}\| L_{L_{p}\left(\Omega_{+}, \mathbb{R}^{n}\right)} \leq\|\mathbf{v}\|_{L_{2 p}\left(\Omega_{+}, \mathbb{R}^{n}\right)}\|\mathbf{w}\|_{L_{2 p}\left(\Omega_{+}, \mathbb{R}^{n}\right)} \leq c_{1}^{\prime}\|\mathbf{v}\|_{B_{p, p^{*}}^{1+\frac{1}{p}}\left(\Omega_{+}, \mathbb{R}^{n}\right)}\|\mathbf{w}\|_{B_{p, p^{*}}^{1+\frac{1}{p}}\left(\Omega_{+}, \mathbb{R}^{n}\right)}, \forall \mathbf{v}, \mathbf{w} \in B_{p, p^{*}}^{1+\frac{1}{p}}\left(\Omega_{+}, \mathbb{R}^{n}\right),
$$

with some constants $c_{k}^{\prime} \equiv c_{k}^{\prime}\left(\Omega_{+}, p\right)>0, k=0,1$, implying that $|\mathbf{v}| \mathbf{w} \in L_{p}\left(\Omega_{+}, \mathbb{R}^{n}\right), \forall \mathbf{v}, \mathbf{w} \in B_{p, p^{*}}^{1+\frac{1}{p}}\left(\Omega_{+}, \mathbb{R}^{n}\right)$.

Next, for a given fixed $\mathbf{v} \in B_{p, p}^{1+\frac{1}{p}}\left(\Omega_{+}, \mathbb{R}^{n}\right)$, we consider the linear Poisson problem of mixed type for the Brinkman system

$$
\left\{\begin{array}{l}
\triangle \mathbf{v}^{0}-\alpha \mathbf{v}^{0}-\nabla \pi^{0}=\mathbf{f}+\beta|\mathbf{v}| \mathbf{v} \text { in } \Omega_{+} \\
\gamma_{+} \mathbf{v}^{0} \mid S_{D}=\mathbf{h}_{0} \in H_{p}^{1}\left(S_{D}, \mathbb{R}^{n}\right) \\
\left.\mathbf{t}_{\alpha}^{+}\left(\mathbf{v}^{0}, \pi^{0}\right)\right|_{S_{N}}=\mathbf{g}_{0} \in L_{p}\left(S_{N}, \mathbb{R}^{n}\right)
\end{array}\right.
$$

with the unknown fields $\left(\mathbf{v}^{0}, \pi^{0}\right) \in B_{p, p^{*}}^{1+\frac{1}{p}}\left(\Omega_{+}, \mathbb{R}^{n}\right) \times B_{p, p^{*}}^{\frac{1}{p}}\left(\Omega_{+}\right)$.

Let $2-\varepsilon<p<2+\varepsilon$, where $\varepsilon>0$ is as in Theorem 6.9 and such that $2-\varepsilon>\frac{3}{2}$. Then by Theorem 6.9, problem (7.9) with given data $\left(\mathbf{f}+\beta|\mathbf{v}| \mathbf{v}, \mathbf{h}_{0}, \mathbf{g}_{0}\right) \in L_{p}\left(\Omega_{+}, \mathbb{R}^{n}\right) \times H_{p}^{1}\left(S_{D}, \mathbb{R}^{n}\right) \times L_{p}\left(S_{N}, \mathbb{R}^{n}\right)$ has a unique solution

$$
\left(\mathbf{v}^{0}, \pi^{0}\right):=(\mathcal{U}(\mathbf{v}), \mathcal{P}(\mathbf{v}))=\mathcal{A}_{p}\left(\mathbf{f}+\beta|\mathbf{v}| \mathbf{v}, \mathbf{h}_{0}, \mathbf{g}_{0}\right) \in \mathcal{X}_{p}
$$

where the linear and continuous operator $\mathcal{A}_{p}: \mathcal{Y}_{p} \rightarrow \mathcal{X}_{p}$ has been defined in Theorem 6.9, and

$$
\mathcal{X}_{p}:=B_{p, p^{*}}^{1+\frac{1}{p}}\left(\Omega_{+}, \mathbb{R}^{n}\right) \times B_{p, p^{*}}^{\frac{1}{p}}\left(\Omega_{+}\right), \quad \mathcal{Y}_{p}:=L_{p}\left(\Omega_{+}, \mathbb{R}^{n}\right) \times H_{p}^{1}\left(S_{D}, \mathbb{R}^{n}\right) \times L_{p}\left(S_{N}, \mathbb{R}^{n}\right) .
$$

Hence, for fixed data $\left(\mathbf{f}, \mathbf{h}_{0}, \mathbf{g}_{0}\right) \in L_{p}\left(\Omega_{+}, \mathbb{R}^{n}\right) \times H_{p}^{1}\left(S_{D}, \mathbb{R}^{n}\right) \times L_{p}\left(S_{N}, \mathbb{R}^{n}\right)$, the nonlinear operators

$$
(\mathcal{U}, \mathcal{P}): B_{p, p^{*}}^{1+\frac{1}{p}}\left(\Omega_{+}, \mathbb{R}^{n}\right) \rightarrow \mathcal{X}_{p}
$$

defined in (7.10), are continuous and bounded, we obtain,

$$
\begin{aligned}
\|(\mathcal{U}(\mathbf{w}), \mathcal{P}(\mathbf{w}))\|_{\mathcal{X}_{p}} & \leq C\left\|\left(\mathbf{f}+\beta|\mathbf{w}| \mathbf{w}, \mathbf{h}_{0}, \mathbf{g}_{0}\right)\right\| \mathcal{Y}_{p} \\
& \leq C\left(\left\|\left(\mathbf{f}, \mathbf{h}_{0}, \mathbf{g}_{0}\right)\right\|_{L_{p}\left(\Omega_{+}, \mathbb{R}^{n}\right) \times H_{p}^{1}\left(S_{D}, \mathbb{R}^{n}\right) \times L_{p}\left(S_{N}, \mathbb{R}^{n}\right)}+\beta\||\mathbf{w}| \mathbf{w}\| L_{L_{p}\left(\Omega_{+}, \mathbb{R}^{n}\right)}\right) \\
& \leq C\left\|\left(\mathbf{f}, \mathbf{h}_{0}, \mathbf{g}_{0}\right)\right\| \mathcal{Y}_{p}+C C_{2}\|\mathbf{w}\|_{B_{p, p^{*}}^{1+\frac{1}{p}}\left(\Omega_{+}, \mathbb{R}^{n}\right)}^{2}, \quad \forall \mathbf{w} \in B_{p, p^{*}}^{1+\frac{1}{p}}\left(\Omega_{+}, \mathbb{R}^{n}\right) \\
\left\|\gamma_{+} \mathcal{U}(\mathbf{w})\right\|_{H_{p}^{1}\left(\partial \Omega, \mathbb{R}^{n}\right)}+\left\|\mathbf{t}_{\alpha}^{+}(\mathcal{U}(\mathbf{w}), \mathcal{P}(\mathbf{w}))\right\|_{\left.L_{p}\left(\partial \Omega, \mathbb{R}^{n}\right)\right)} & \leq C^{\prime}\left\|\left(\mathbf{f}, \mathbf{h}_{0}, \mathbf{g}_{0}\right)\right\|_{\mathcal{Y}_{p}}+C^{\prime} C_{2}\|\mathbf{w}\|_{B_{p, p^{*}}^{1+\frac{1}{p}}\left(\Omega_{+}, \mathbb{R}^{n}\right)}^{2}
\end{aligned}
$$

where $C_{2}:=c_{1}^{\prime} \beta>0$, and $c_{1}^{\prime} \equiv c_{1}^{\prime}\left(\Omega_{+}, p\right)>0$ is the constant that appears in inequality (7.8), and $C$ can be taken as $C=\left\|\mathcal{A}_{p}\right\|_{\mathcal{L}\left(\mathcal{Y}_{p}, \mathcal{X}_{p}\right)}$. In addition, in view of (7.10) and due to the definition of $\mathcal{A}_{p}$, we obtain that $\left(\mathbf{v}^{0}, \pi^{0}\right)=(\mathcal{U}(\mathbf{v}), \mathcal{P}(\mathbf{v}))$ and satisfy (7.9). Therefore, if we show that the nonlinear operator $\mathcal{U}$ has a fixed point $\mathbf{u} \in B_{p, p^{*}}^{1+\frac{1}{p}}\left(\Omega_{+}, \mathbb{R}^{n}\right)$, i.e., such that $\mathcal{U}(\mathbf{u})=\mathbf{u}$, then $\mathbf{u}$ together with the pressure function $\pi=\mathcal{P}(\mathbf{u})$ determine a solution of the nonlinear mixed problem (7.3) in the space $\mathcal{X}_{p}$. In order to show the existence of such a fixed point, we introduce the constants

$$
\zeta_{p}:=\frac{3}{16 C_{2} C^{2}}>0, \quad \eta_{p}:=\frac{1}{4 C_{2} C}>0
$$

(cf. [32, Theorem 5.2]) and the closed ball

$$
\mathbf{B}_{\eta_{p}}:=\left\{\mathbf{w} \in B_{p, p^{*}}^{1+\frac{1}{p}}\left(\Omega_{+}, \mathbb{R}^{n}\right):\|\mathbf{w}\|_{B_{p, p^{*}}^{1+\frac{1}{p}}\left(\Omega_{+}, \mathbb{R}^{n}\right)} \leq \eta_{p}\right\}
$$

and assume that the given data satisfy the inequality

$$
\left\|\left(\mathbf{f}, \mathbf{h}_{0}, \mathbf{g}_{0}\right)\right\|_{\mathcal{Y}_{p}} \leq \zeta_{p}
$$


Then by (7.13), (7.15)-(7.17) we deduce that

$$
\|(\mathcal{U}(\mathbf{w}), \mathcal{P}(\mathbf{v}))\|_{\mathcal{X}_{p}} \leq \frac{1}{4 C_{2} C}=\eta_{p}, \forall \mathbf{w} \in \mathbf{B}_{\eta_{p}}
$$

Consequently, $\mathcal{U}$ maps $\mathbf{B}_{\eta_{p}}$ into $\mathbf{B}_{\eta_{p}}$.

Moreover, we now prove that $\mathcal{U}$ is a contraction on $\mathbf{B}_{\eta_{p}}$. Indeed, by using the expression of $\mathcal{U}$ given in (7.10), the linearity and continuity of the operator $\mathcal{A}_{p}$, and inequality (7.8), we obtain that

$$
\begin{aligned}
&\|\mathcal{U}(\mathbf{v})-\mathcal{U}(\mathbf{w})\|_{B_{p, p^{*}}^{1+\frac{1}{p}}\left(\Omega_{+}, \mathbb{R}^{n}\right)} \leq\left\|\mathcal{A}_{p}(\beta|\mathbf{v}| \mathbf{v}-\beta|\mathbf{w}| \mathbf{w}, \mathbf{0}, \mathbf{0})\right\|_{B_{p, p^{*}}^{1+\frac{1}{p}}\left(\Omega_{+}, \mathbb{R}^{n}\right)} \\
& \leq C \beta\||\mathbf{v}| \mathbf{v}-|\mathbf{w}| \mathbf{w}\|_{L_{p}\left(\Omega_{+}, \mathbb{R}^{n}\right)}=C \beta\|(|\mathbf{v}|-|\mathbf{w}|) \mathbf{v}+|\mathbf{w}|(\mathbf{v}-\mathbf{w})\| \|_{L_{p}\left(\Omega_{+}, \mathbb{R}^{n}\right)} \\
& \leq C C_{1}^{\prime} \beta\left(\|\mathbf{v}\|_{B_{p, p^{*}}^{1+\frac{1}{p}}\left(\Omega_{+}, \mathbb{R}^{n}\right)}+\|\mathbf{w}\|_{B_{p, p^{*}}^{1+\frac{1}{p}}\left(\Omega_{+}, \mathbb{R}^{n}\right)}\right)\|\mathbf{v}-\mathbf{w}\|_{B_{p, p^{*}}^{1+\frac{1}{p}}\left(\Omega_{+}, \mathbb{R}^{n}\right)} \\
&\left.\leq 2 \eta_{p} C C_{2}\|\mathbf{v}-\mathbf{w}\|_{B_{p, p^{*}}^{1+\frac{1}{p}}\left(\Omega_{+}, \mathbb{R}^{n}\right)}=\frac{1}{2}\|\mathbf{v}-\mathbf{w}\|_{B_{p, p^{*}}^{1+\frac{1}{p}}}, \forall \Omega_{+}, \mathbb{R}^{n}\right) \\
&
\end{aligned}
$$

see also (7.13). Then the Banach-Caccioppoli fixed point theorem implies that there exists a unique fixed point $\mathbf{u} \in \mathbf{B}_{\eta_{p}}$ of $\mathcal{U}$, i.e., $\mathcal{U}(\mathbf{u})=\mathbf{u}$. Moreover, $\mathbf{u}$ and the pressure function $\pi=\mathcal{P}(\mathbf{u})$, given by (7.10), determine a solution of the semilinear problem (7.3) in the space $B_{p, p^{*}}^{1+\frac{1}{p}}\left(\Omega_{+}, \mathbb{R}^{n}\right) \times B_{p, p^{*}}^{\frac{1}{p}}\left(\Omega_{+}\right)$. In addition, since the solution satisfies the condition $\mathbf{u} \in \mathbf{B}_{\eta}$, by inequality (7.13) we obtain the estimate

$$
\|\mathbf{u}\|_{\substack{B_{p, p^{*}}^{1+\frac{1}{p}}\left(\Omega_{+}, \mathbb{R}^{n}\right) \\ B_{p, p^{*}}}}+\|\pi\|_{\left.\Omega_{+}^{\frac{1}{p}}\right)} \leq C\left\|\left(\mathbf{f}, \mathbf{h}_{0}, \mathbf{g}_{0}\right)\right\| \mathcal{Y}_{p}+\frac{1}{4}\|\mathbf{u}\|_{B_{p, p^{*}}^{1+\frac{1}{p}}\left(\Omega_{+}, \mathbb{R}^{n}\right)},
$$

implying that

$$
\|\mathbf{u}\|_{B_{p, p^{*}}^{1+\frac{1}{p}}\left(\Omega_{+}, \mathbb{R}^{n}\right)}+\|\pi\|_{B_{p, p^{*}}^{\frac{1}{p}}\left(\Omega_{+}\right)} \leq \frac{4}{3} C\left\|\left(\mathbf{f}, \mathbf{h}_{0}, \mathbf{g}_{0}\right)\right\| \mathcal{Y}_{p},
$$

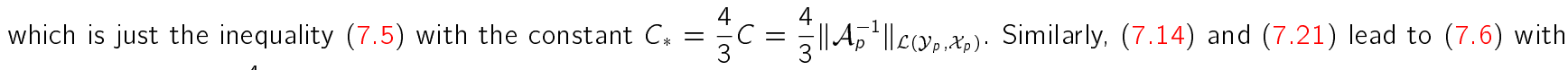
the constant $C_{*}^{\prime}=\frac{4}{3} C^{\prime}$

Next, we prove the uniqueness of the semilinear mixed problem (7.3) solution $(\mathbf{u}, \pi) \in \mathcal{X}_{p}$, that satisfies inequality (7.4), when the given data satisfy conditions (7.2). Assume that $\left(\mathbf{u}^{\prime}, \pi^{\prime}\right) \in \mathcal{X}_{p}$ is another solution of problem (7.3), which satisfies inequality (7.4), implying $\mathbf{u}^{\prime} \in \mathbf{B}_{\eta_{p}}$. Then $\mathcal{U}\left(\mathbf{u}^{\prime}\right) \in \mathbf{B}_{\eta_{p}}$, where $\left(\mathcal{U}\left(\mathbf{u}^{\prime}\right), \mathcal{P}\left(\mathbf{u}^{\prime}\right)\right)$ are given by (7.10) and satisfy (7.9) with $\mathbf{v}$ replaced by $\mathbf{u}^{\prime}$. Then by (7.3) and (7.21) (both written in terms of $\left(\mathbf{u}^{\prime}, \boldsymbol{\pi}^{\prime}\right)$ ) we obtain the linear mixed Dirichlet-Neumann problem

$$
\left\{\begin{array}{l}
\triangle\left(\mathcal{U}\left(\mathbf{u}^{\prime}\right)-\mathbf{u}^{\prime}\right)-\alpha\left(\mathcal{U}\left(\mathbf{u}^{\prime}\right)-\mathbf{u}^{\prime}\right)-\nabla\left(\mathcal{P}\left(\mathbf{u}^{\prime}\right)-\pi^{\prime}\right)=\mathbf{0} \text { in } \Omega_{+}, \\
\left.\left(\gamma_{+}\left(\mathcal{U}\left(\mathbf{u}^{\prime}\right)-\mathbf{u}^{\prime}\right)\right)\right|_{S_{D}}=\mathbf{0} \text { on } S_{D}, \\
\left.\left(\mathbf{t}_{\alpha}^{+}\left(\mathcal{U}\left(\mathbf{u}^{\prime}\right)-\mathbf{u}^{\prime}, \mathcal{P}\left(\mathbf{u}^{\prime}\right)-\pi^{\prime}\right)\right)\right|_{S_{N}}=\mathbf{0} \text { on } S_{N},
\end{array}\right.
$$

and $\gamma_{+}\left(\mathcal{U}\left(\mathbf{u}^{\prime}\right)-\mathbf{u}^{\prime}\right) \in H_{p}^{1}\left(\partial \Omega_{+}, \mathbb{R}^{n}\right), \mathbf{t}_{\alpha}^{+}\left(\mathcal{U}\left(\mathbf{u}^{\prime}\right)-\mathbf{u}^{\prime}, \mathcal{P}\left(\mathbf{u}^{\prime}\right)-\pi^{\prime}\right) \in L_{p}\left(\partial \Omega_{+}, \mathbb{R}^{n}\right)$. This problem has only the trivial solution in the space $\mathcal{X}_{p}$ (see Theorem 6.9), i.e., $\mathcal{U}\left(\mathbf{u}^{\prime}\right)=\mathbf{u}^{\prime}, \mathcal{P}\left(\mathbf{u}^{\prime}\right)=\pi^{\prime}$. Thus, $\mathbf{u}^{\prime}$ is a fixed point of $\mathcal{U}$. Since $\mathcal{U}: \mathbf{B}_{\eta_{p}} \rightarrow \mathbf{B}_{\eta_{p}}$ is a contraction, it has a unique fixed point in $\mathbf{B}_{\eta_{p}}$, which has been already denoted by $\mathbf{u}$. Consequently, $\mathbf{u}^{\prime}=\mathbf{u}$, and, in addition, $\pi^{\prime}=\pi$.

\section{Appendices}

\section{A. Besov spaces in $\mathbb{R}^{n}$}

Let $\mu=\left(\mu_{1}, \ldots, \mu_{n}\right)$ be an arbitrary multi-index in $\mathbb{Z}_{+}^{n}$ of length $|\mu|:=\mu_{1}+\cdots+\mu_{n}$, and let $\partial^{\mu}:=\frac{\partial^{|\mu|}}{\partial x_{1}^{\mu_{1}} \cdots \partial x_{n}^{\mu_{n}}}$. Next we recall the definition of Besov spaces in $\mathbb{R}^{n}$ (cf., e.g., [61, Section 11.1]). By $三$ one denotes the collection of all sets $\left\{\xi_{j}\right\}_{j=0}^{\infty}$ of Schwartz functions with the following property:

(i) There are some constants $b, c, d>0$ such that

$$
\operatorname{supp}\left(\xi_{0}\right) \subset\{x:|x| \leq b\}, \operatorname{supp}\left(\xi_{j}\right) \subset\left\{x: 2^{j-1} c \leq|x| \leq 2^{j+1} d\right\}, j=1,2, \ldots
$$

(ii) Let $\mu$ be an arbitrary multi-index in $\mathbb{R}^{n}$. Then there exists a constant $c_{\partial \Omega}>0$ such that

$$
\sup _{x \in \mathbb{R}^{n}} \sup _{j \in \mathbb{N}} 2^{j|\mu|}\left|\partial^{\mu} \xi_{j}(x)\right| \leq c_{\partial \Omega} .
$$


(iii) The following equality holds

$$
\sum_{j=0}^{\infty} \xi_{j}(x)=1, \forall x \in \mathbb{R}^{n}
$$

Let $s \in \mathbb{R}, p, q \in(0, \infty)$. Then for a sequence $\left\{\xi_{j}\right\}_{j=0}^{\infty} \subset \equiv$, the Besov space $B_{p, q}^{s}\left(\mathbb{R}^{n}\right)$ is defined by

$$
B_{p, q}^{s}\left(\mathbb{R}^{n}\right):=\left\{f \in \mathcal{S}^{\prime}\left(\mathbb{R}^{n}\right):\|f\|_{B_{p, q}^{s}\left(\mathbb{R}^{n}\right)}:=\left(\sum_{j=1}^{\infty}\left\|2^{s j} \mathcal{F}^{-1}\left(\xi_{j} \mathcal{F} f\right)\right\|_{L_{p}\left(\mathbb{R}^{n}\right)}^{q}\right)^{\frac{1}{q}}<\infty\right\},
$$

where $\mathbf{f}$ is the Fourier transform and $\mathcal{S}^{\prime}\left(\mathbb{R}^{n}\right)$ denotes the space of tempered distributions in $\mathbb{R}^{n}$. Note that the above definition of the Besov space $B_{p, q}^{s}\left(\mathbb{R}^{n}\right)$ is independent of the choice of the set $\left\{\xi_{j}\right\}_{j=0}^{\infty} \subset \equiv$, which means that another sequence in $\equiv$ leads to the same space with an equivalent norm. In particular, for any $s \in \mathbb{R}$, the Besov space $B_{2,2}^{s}\left(\mathbb{R}^{n}\right)$ coincides with the Sobolev space $H_{2}^{s}\left(\mathbb{R}^{n}\right)$, i.e., $B_{2,2}^{s}\left(\mathbb{R}^{n}\right)=H_{2}^{s}\left(\mathbb{R}^{n}\right)$. Moreover, denoting by $W_{p}^{s}\left(\mathbb{R}^{n}\right)$ the Sobolev-Slobodeckij spaces (defined in the classical way through their norms), we have the relations (see, e.g., [72], [5])

$$
\begin{aligned}
& W_{p}^{s}\left(\mathbb{R}^{n}\right)=B_{p, p}^{s}\left(\mathbb{R}^{n}\right), \quad s \in \mathbb{R} \backslash \mathbb{Z}, \\
& W_{p}^{k}\left(\mathbb{R}^{n}\right)=H_{p}^{k}\left(\mathbb{R}^{n}\right), \quad k \in \mathbb{Z} .
\end{aligned}
$$

Let $s_{0}, s_{1} \in \mathbb{R}, 1<p_{0} \leq p_{1}<\infty$ be such that $s_{1}-\frac{n}{p_{1}}<s_{0}-\frac{n}{p_{0}}$, and $0<q_{0}, q_{1} \leq \infty$. Then the embedding

$$
B_{p_{0}, q_{0}}^{s_{0}}\left(\mathbb{R}^{n}\right) \hookrightarrow B_{p_{1}, q_{1}}^{s_{1}}\left(\mathbb{R}^{n}\right)
$$

is continuous (cf. [72, Theorem in Section 2.7.1 and Proposition 2(ii) in Section 2.3.2], [66, Remark 2 in Section 2.2.3]). Note that $\mathbb{R}^{n}$ in (A.7) can be replaced by a domain $\Omega \in \mathbb{R}^{n}$.

Let us also recall the following useful inclusions between Besov spaces and Bessel potential spaces. Assume that $1 \leq q_{1} \leq$ $q_{2} \leq \infty, 1 \leq p, q \leq \infty$ and $s_{1}<s<s_{2}$. Let $p^{\prime}$ denote the conjugate exponent of $p$, i.e., $\frac{1}{p^{\prime}}=1-\frac{1}{p}$. Then we have the following continuous embeddings,

$$
\begin{aligned}
& B_{p, q_{1}}^{s}\left(\mathbb{R}^{n}\right) \hookrightarrow B_{p, q_{2}}^{s}\left(\mathbb{R}^{n}\right), \quad B_{p, \min \left\{p, p^{\prime}\right\}}^{s}\left(\mathbb{R}^{n}\right) \hookrightarrow H_{p}^{s}\left(\mathbb{R}^{n}\right) \hookrightarrow B_{p, \max \left\{p, p^{\prime}\right\}}^{s}\left(\mathbb{R}^{n}\right) \\
& B_{2,2}^{s}\left(\mathbb{R}^{n}\right)=H_{2}^{s}\left(\mathbb{R}^{n}\right), \quad B_{p, \infty}^{s_{2}}\left(\mathbb{R}^{n}\right) \hookrightarrow H_{p}^{s}\left(\mathbb{R}^{n}\right) \hookrightarrow B_{p, 1}^{s_{1}}\left(\mathbb{R}^{n}\right),
\end{aligned}
$$

(cf., e.g., [3, Chapter 6], [71, (3.2)], [62, (4.19)]), which imply the continuity of the embedding

$$
B_{p, q}^{s_{2}}\left(\mathbb{R}^{n}\right) \hookrightarrow B_{p, q}^{s_{1}}\left(\mathbb{R}^{n}\right)
$$

These embeddings hold also when $\mathbb{R}^{n}$ is replaced by a bounded Lipschitz domain (see [3, Chapter 6], [73, (8)]).

The scales of Bessel potential and Besov spaces can be obtained by the method of complex interpolation. Indeed, if $s_{0}, s_{1} \in \mathbb{R}$, $s_{0} \neq s_{1}, p_{0}, p_{1} \in(1,+\infty), q_{0}, q_{1} \in(1,+\infty)$ and $\theta \in(0,1)$, then (cf., e.g., [72], [61, Theorem 11.1.2], [5, Theorem 3.1]):

$$
\left[H_{p_{0}}^{s_{0}}\left(\mathbb{R}^{n}\right), H_{p_{1}}^{s_{1}}\left(\mathbb{R}^{n}\right)\right]_{\theta}=H_{p}^{s}\left(\mathbb{R}^{n}\right), \quad\left[B_{p_{0}, q_{0}}^{s_{0}}\left(\mathbb{R}^{n}\right), B_{p_{1}, q_{1}}^{s_{1}}\left(\mathbb{R}^{n}\right)\right]_{\theta}=B_{p, q}^{s}\left(\mathbb{R}^{n}\right)
$$

where $s=(1-\theta) s_{0}+\theta s_{1}, \frac{1}{p}=\frac{1-\theta}{p_{0}}+\frac{\theta}{p_{1}}$ and $\frac{1}{q}=\frac{1-\theta}{q_{0}}+\frac{\theta}{q_{1}}$.

Moreover, the scale of Besov spaces can be also obtained by using the method of real interpolation of Sobolev spaces. Indeed, for $p, q \in(1,+\infty), s_{0} \neq s_{1}$, and $\theta \in(0,1)$, we have the following real interpolation property

$$
\left(H_{p}^{s_{1}}\left(\mathbb{R}^{n}\right), H_{p}^{s_{2}}\left(\mathbb{R}^{n}\right)\right)_{\theta, q}=B_{p, q}^{s}\left(\mathbb{R}^{n}, \mathbb{R}^{n}\right),
$$

where $s=(1-\theta) s_{0}+\theta s_{1}$ (cf., e.g., [1, Theorem 14.1.5], [24, p. 329], [29], [57, (5.38)], [72], [5, Theorem 3.1]).

Formulas (A.11) and (A.12) remain true if $\mathbb{R}^{n}$ is replaced by a Lipschitz domain (cf., e.g., [5, Theorem 3.2, Remark 3.3]).

For the following property we refer the reader to, e.g., [57, relations (3.11) and Proposition 4.2].

Lemma A.1 Let $\Omega \subset \mathbb{R}^{n}$ be a bounded Lipschitz domain. Let $S \subset \partial \Omega$ be an admissible patch. If $p_{0}, p_{1} \in(1, \infty), s_{0}, s_{1} \in[0,1]$ or $s_{0}, s_{1} \in[-1,0]$, and $\theta \in(0,1)$, then the following complex and real interpolation properties hold

$$
\begin{aligned}
& {\left[H_{p_{0}}^{s_{0}}(\partial \Omega), H_{p_{1}}^{s_{1}}(\partial \Omega)\right]_{\theta}=H_{p}^{s}(\partial \Omega), \quad\left[H_{p_{0}}^{s_{0}}(S), H_{p_{1}}^{s_{1}}(S)\right]_{\theta}=H_{p}^{s}(S), \quad\left[\widetilde{H}_{p_{0}}^{s_{0}}(S), \widetilde{H}_{p_{1}}^{s_{1}}(S)\right]_{\theta}=\widetilde{H}_{p}^{s}(S),} \\
& \left(H_{p_{0}}^{s_{0}}(\partial \Omega), H_{p_{1}}^{s_{1}}(\partial \Omega)\right)_{\theta, q}=B_{p, q}^{s}(\partial \Omega), \quad\left(H_{p_{0}}^{s_{0}}(S), H_{p_{1}}^{s_{1}}(S)\right)_{\theta, q}=B_{p, q}^{s}(S), \quad\left[\widetilde{H}_{p_{0}}^{s_{0}}(S), \widetilde{H}_{p_{1}}^{s_{1}}(S)\right]_{\theta, q}=\widetilde{B}_{p, q}^{s}(S),
\end{aligned}
$$

where $\frac{1}{p}=\frac{1-\theta}{p_{0}}+\frac{\theta}{p_{1}}$ and $s=(1-\theta) s_{0}+\theta s_{1} . \ln (\mathrm{A} .14)$ also $s_{0} \neq s_{1}$ and $q \in(1, \infty]$. 


\section{B. Some general assertions on interpolation theory and continuous operators}

Let us consider two compatible couples of Banach spaces, $X_{0}, X_{1}$ and $Y_{0}, Y_{1}$. Let $X_{\theta}$ and $Y_{\theta}$ be interpolation spaces with respect to $X_{0}, X_{1}$ and $Y_{0}, Y_{1}$, according to [3, Definition 2.4.1]. If $A_{j}: X_{j} \rightarrow Y_{j}, j=0,1$ are linear continuous compatible operators (i.e., $\left.A_{0}\left|x_{0} \cap X_{1}=A_{1}\right| x_{0} \cap X_{1}\right)$ then they induce the operator $A_{+}: X_{0}+X_{1} \rightarrow Y_{0}+Y_{1}$, such that $A_{+} x:=A_{0} x_{0}+A_{1} x_{1}$, for any $x \in X_{0}+X_{1}$, where $x=x_{0}+x_{1}, x_{j} \in X_{j}$, and $\left\|A_{+}\right\| \leq \max \left(\left\|A_{0}\right\|,\left\|A_{1}\right\|\right)$, cf. [3, Section 2.3, Eq. (3)]. Further, $X_{\theta} \subset X_{0}+X_{1}$ and the operator $A_{\theta}:=A_{+} \mid x_{\theta}$ is linear and continuous. In the following assertion we consider some cases when the interpolation preserves isomorphism properties of operators.

Lemma B.1 Let $X_{0}, X_{1}$ and $Y_{0}, Y_{1}$ be two compatible couples of Banach spaces. Let $X_{\theta}$ and $Y_{\theta}$ be interpolation spaces with respect to $X_{0}, X_{1}$ and $Y_{0}, Y_{1}$. Let $A_{j}: X_{j} \rightarrow Y_{j}, j=0,1$, be linear continuous compatible operators that are isomorphisms. Let $A_{\theta}: X_{\theta} \rightarrow Y_{\theta}$ be the operator induced by $A_{j}$.

(i) If the operators $R_{j}: Y_{j} \rightarrow X_{j}$, inverse to the operators $A_{j}: X_{j} \rightarrow Y_{j}, j=0,1$, respectively, are compatible (i.e., $R_{0} \mid Y_{0} \cap Y_{1}=$ $\left.R_{1} \mid Y_{0} \cap Y_{1}\right)$, then $A_{\theta}: X_{\theta} \rightarrow Y_{\theta}$ is an isomorphism

(ii) If $X_{0} \subset X_{1}$, then $A_{\theta}: X_{\theta} \rightarrow Y_{\theta}$ is an isomorphism.

(iii) If there exist linear subspaces $X_{*} \subset X_{0} \cap X_{1}$ and $Y_{*} \subset Y_{0} \cap Y_{1}$ such that $Y_{*}$ is dense in $Y_{0} \cap Y_{1}$ and the operator $A_{*}:=\left.A_{0}\right|_{X_{*}}=\left.A_{1}\right|_{X_{*}}: X_{*} \rightarrow Y_{*}$ is an isomorphism, then $A_{\theta}: X_{\theta} \rightarrow Y_{\theta}$ is an isomorphism.

Proof. Let us prove item (i). Since the inverse operators $R_{j}: Y_{j} \rightarrow X_{j}$ are compatible, they induce a continuous operator $R_{+}: Y_{0}+Y_{1} \rightarrow X_{0}+X_{1}$, such that $R_{+} y:=R_{0} y_{0}+R_{1} y_{1}$, for any $y \in Y_{0}+Y_{1}$, where $y=y_{0}+y_{1}, y_{j} \in Y_{j}$, and continuous operator $R_{\theta}=R_{+} \mid Y_{\theta}: Y_{\theta} \rightarrow X_{\theta}$. Let us show that the operator $R_{\theta}$ is inverse to $A_{\theta}$. Indeed, any $x \in X_{\theta}$ can be represented as $x=x_{0}+x_{1}$, where $x_{j} \in X_{j}$, and then

$$
R_{\theta} A_{\theta} x=R_{+} A_{+} x=R_{+} A_{+}\left(x_{0}+x_{1}\right)=R_{+}\left(A_{0} x_{0}+A_{1} x_{1}\right)=R_{0} A_{0} x_{0}+R_{1} A_{1} x_{1}=x_{0}+x_{1}=x .
$$

Similarly, any $y \in Y_{\theta}$ can be represented as $y=y_{0}+y_{1}$, where $y_{j} \in Y_{j}$, and then

$$
A_{\theta} R_{\theta} y=A_{+} R_{+} y=A_{+} R_{+}\left(y_{0}+y_{1}\right)=A_{+}\left(R_{0} y_{0}+R_{1} y_{1}\right)=A_{0} R_{0} y_{0}+A_{1} R_{1} y_{1}=y_{0}+y_{1}=y .
$$

This proves that $R_{\theta}: Y_{\theta} \rightarrow X_{\theta}$ is the operator inverse to $A_{\theta}: X_{\theta} \rightarrow Y_{\theta}$ and hence the latter one is an isomorphism.

To prove item (ii) we remark that the inclusion $X_{0} \subset X_{1}$, the compatibility of the operators $A_{j}: X_{j} \rightarrow Y_{j}, j=0,1$, and the invertibility of the operator $A_{0}: X_{0} \rightarrow Y_{0}$ imply that $Y_{0} \subset Y_{1}$. Then the invertibility of the operator $A_{1}: X_{1} \rightarrow Y_{1}$ implies $R_{1} \mid Y_{0}=R_{0}$, i.e., the compatibility of the inverse operators to the operators $A_{j}: X_{j} \rightarrow Y_{j}, j=0,1$, which reduces item (ii) to item (i).

Let us prove item (iii). If $A_{j}: X_{j} \rightarrow Y_{j}, j=0,1$, are isomorphisms then there exist continuous inverse operators $R_{j}: Y_{j} \rightarrow X_{j}$, $j=0,1$. Let us prove that $R_{j}$ are compatible operators. Let $R_{*}: Y_{*} \rightarrow X_{*}$ be the inverse to the operator $A_{*}:=A_{0}\left|X_{*}=A_{1}\right|_{X_{*}}:$ $X_{*} \rightarrow Y_{*}$. Then for any $\psi \in Y_{*}$, there exists $\phi \in X_{*}$ such that $\psi=A_{*} \phi=A_{0} \phi=A_{1} \phi$. Hence $R_{*} \psi=\phi=R_{0} \psi=R_{1} \psi$, i.e., $R_{*}=R_{0}\left|Y_{*}=R_{1}\right| Y_{*}$

Due to the density of $Y_{*}$ in $Y_{0} \cap Y_{1}$, for any $y \in Y_{0} \cap Y_{1}$ there exists a sequence $\left\{\psi^{i}\right\}_{i=1}^{\infty} \subset Y_{*}$ converging to $y$ in $Y_{0} \cap Y_{1}$ and hence in $Y_{0}$ and in $Y_{1}$. Then $R_{*} \psi^{i} \in X_{*} \subset X_{0} \cup X_{1}$ and due to continuity of the operators $R_{j}: Y_{j} \rightarrow X_{j}, j=0,1$, $\lim _{i \rightarrow \infty} R_{*} \psi^{i}=\lim _{i \rightarrow \infty} R_{j} \psi^{i}=R_{j} y$ in $X_{j}$ for $j=0,1$, which implies $R_{1}\left|Y_{0} \cap Y_{1}=R_{2}\right| Y_{0} \cap Y_{1}$, i.e., the inverse operators $R_{j}: Y_{j} \rightarrow X_{j}$, $j=0,1$ are compatible.

Employing now item (i) concludes the proof of item (iii).

Note that item (iii) of Lemma B.1 is available in [24, Lemma 8.4] for the cases, when the image and domain spaces coincide, i.e, $X_{j}=Y_{j}$, under the additional assumptions that $X_{*}=Y_{*}$ is a Banach space.

Let us give the following useful result in the complex interpolation theory (cf., e.g., [12, Theorem 2.7, Corollary 2.8] and the references therein, see also [44, Appendix B]).

Lemma B.2 Let $X_{0}, X_{1}$ and $Y_{0}, Y_{1}$ be two compatible couples of Banach spaces and $A_{j}: X_{j} \rightarrow Y_{j}, j=0,1$, be two continuous compatible linear operators. Let $X_{\theta}:=\left[X_{0}, X_{1}\right]_{\theta}$ and $Y_{\theta}:=\left[Y_{0}, Y_{1}\right]_{\theta}$ denote the complex interpolation spaces of $X_{0}, X_{1}$ and $Y_{0}, Y_{1}$, respectively, for each $\theta \in(0,1)$. If there exists a number $\theta_{0} \in(0,1)$ such that $A_{\theta_{0}}: X_{\theta_{0}} \rightarrow Y_{\theta_{0}}$ is an isomorphism, then there exists $\varepsilon>0$ such that the operator $A_{\theta}: X_{\theta} \rightarrow Y_{\theta}$ is an isomorphism as well, for any $\theta \in\left(\theta_{0}-\varepsilon, \theta_{0}+\varepsilon\right)$.

Remark B.3 The extension of Lemma B.2 to the case of two compatible couples of quasi-Banach spaces, $X_{0}, X_{1}$ and $Y_{0}, Y_{1}$, such that $X_{0}+X_{1}$ and $Y_{0}+Y_{1}$ are analytically convex can be found in [61, Theorem 11.9.24] and the references therein. Note that any Banach space is analytically convex (cf., e.g., [61, p.223]).

Finally, let us mention the following useful result (cf, e.g., [61, Lemma 11.9.21]).

Lemma B.4 Let $X_{1}, X_{2}$ and $Y_{1}, Y_{2}$, be Banach spaces such that the embeddings $X_{1} \hookrightarrow X_{2}$ and $Y_{1} \hookrightarrow Y_{2}$ are continuous, and also that the embedding $Y_{1} \hookrightarrow Y_{2}$ has dense range. Assume that $T: X_{1} \rightarrow Y_{1}$ and $T: X_{2} \rightarrow Y_{2}$ are Fredholm operators with the same index, ind $\left(T: X_{1} \rightarrow Y_{1}\right)=$ ind $\left(T: X_{2} \rightarrow Y_{2}\right)$. Then $\operatorname{Ker}\left\{T: X_{1} \rightarrow Y_{1}\right\}=\operatorname{Ker}\left\{T: X_{2} \rightarrow Y_{2}\right\}$. 


\section{References}

1. M.S. Agranovich, Sobolev Spaces, Their Generalizations, and Elliptic Problems in Smooth and Lipschitz Domains, Springer, Heidelberg, 2015.

2. C. Amrouche, M.A. Rodríguez-Bellido, Stationary Stokes, Oseen and Navier-Stokes equations with singular data, Arch. Rational Mech. Anal. 199 (2011), 597-651.

3. J. Bergh and J. Löfström, Interpolation Spaces, An Introduction. Springer-Verlag, Berlin, 1976.

4. C. Băcuță, M.E. Hassell, G.C. Hsiao, F-J. Sayas, Boundary integral solvers for an evolutionary exterior Stokes problem. SIAM J. Numer. Anal. 53 (2015), 1370-1392.

5. A. Behzadan, M. Holst, Multiplication in Sobolev spaces, Revisited. ArXiv:1512.07379v1.

6. K. Brewster, D. Mitrea, I. Mitrea, M. Mitrea, Extending Sobolev functions with partially vanishing traces from locally $(\varepsilon, \delta)-$ domains and applications to mixed boundary problems. J. Functional Analysis. 266 (2014), 4314-4421.

7. R.M. Brown, The mixed problem for Laplaces equation in a class of Lipschitz domains. Comm. Partial Diff. Eqns. 19 (1994), 1217-1233.

8. R.M. Brown, I. Mitrea, The mixed problem for the Lamé system in a class of Lipschitz domains, J. Diff. Equ. 246 (2009), $2577-2589$.

9. R.M. Brown, I. Mitrea, M. Mitrea and M. Wright, Mixed boundary value problems for the Stokes system, Trans. Amer. Math. Soc. 362 (2010), 1211-1230.

10. A. Buffa, G. Geymonat, On traces of functions in $W^{2, p}(\Omega)$ for Lipschitz domains in $\mathbb{R}^{3}$, C. R. Acad. Sci. Paris, Série I, 332 (2001), 699-704.

11. F. Cakoni, G.C. Hsiao, W.L. Wendland, On the boundary integral equation method for a mixed boundary value problem of the biharmonic equation, Complex Var. 50 (2005), 681-696.

12. W. Cao, Y. Sagher, Stability of Fredholm properties on interpolation scales, Ark. Mat. 28 (1990), 249-258.

13. O. Chkadua, S.E. Mikhailov, and D. Natroshvili, Analysis of direct boundary-domain integral equations for a mixed BVP with variable coefficient, I: Equivalence and invertibility, J. Integral Equations Appl. 21 (2009), 499-543.

14. O. Chkadua, S. E. Mikhailov, and D. Natroshvili, Analysis of segregated boundary-domain integral equations for variable-coefficient problems with cracks, Numer. Methods Partial Differential Equations, 27 (2011), 121-140.

15. O. Chkadua, S. E. Mikhailov, D. Natroshvili, Analysis of direct segregated boundary-domain integral equations for variable-coefficient mixed BVPs in exterior domains. Anal. Appl. 11 (2013), no. 4, 1350006.

16. H.J. Choe, H. Kim, Dirichlet problem for the stationary Navier-Stokes system on Lipschitz domains. Commun. Partial Differ. Equ. 36 (2011), 1919-1944.

17. R. Coifman, A. Mclntosh, Y. Meyer, L'Intégrale de Cauchy définit un opérateur borné sur $L^{2}$ pour les courbes Lipschitziennes [The Cauchy integral defines a bounded operator on $L^{2}$ for Lipschitz curves], Ann. Math. 116 (1982), 361-387.

18. M. Costabel, Boundary integral operators on Lipschitz domains: Elementary results, SIAM J. Math. Anal. 19 (1988), $613-626$.

19. M. Costabel, E. Stephan, Boundary integral equations for mixed boundary value problems in polygonal domains and Galerkin approximation. Math. Models Methods Mech. 15 (1985), 175-251.

20. P. Deuring, The Resolvent problem for the Stokes system in exterior domains: An elementary approach, Math. Meth. Appl. Sci. 13 (1990), 335-349.

21. M. Dindoš, M. Mitrea, Semilinear Poisson problems in Sobolev-Besov spaces on Lipschitz domains, Publ. Math. 46 (2002), $353-403$.

22. M. Dindos̆, M. Mitrea, The stationary Navier-Stockes system in nonsmooth manifolds: the Poisson problem in Lipschitz and $C^{1}$ domains, Arch. Rational Mech. Anal. 174 (2004), 1-47.

23. E. Fabes, C. Kenig, G. Verchota, The Dirichlet problem for the Stokes system on Lipschitz domains, Duke Math. J. 57 (1988), 769-793.

24. E. Fabes, O. Mendez, M. Mitrea, Boundary layers on Sobolev-Besov spaces and Poisson's equation for the Laplacian in Lipschitz domains, J. Funct. Anal. 159 (1998), 323-368.

25. T. Groșan, M. Kohr, W.L. Wendland, Dirichlet problem for a nonlinear generalized Darcy-Forchheimer-Brinkman system in Lipschitz domains, Math. Meth. Appl. Sci. 38 (2015), 3615-3628.

26. R. Gutt, T. Groșan, On the Lid-driven problem in a porous cavity. A numerical approach, Appl. Math. Comput. 266 (2015), $1070-1082$.

27. G.C. Hsiao, W.L. Wendland, Boundary Integral Equations. Springer-Verlag, Heidelberg, 2008.

28. S. Hofmann, M. Mitrea, M. Taylor, Symbol calculus for operators of layer potential type on Lipschitz surfaces with VMO normals, and related pseudodifferential operator calculus, Analysis \& PDE. 8 (2015), 115-181.

29. D.S. Jerison, C. Kenig, The inhomogeneous Dirichlet problem in Lipschitz domains, J. Funct. Anal. 130 (1995), 161-219.

30. A. Jonsson, H. Wallin, Function spaces on subsets of $\mathbb{R}^{n}$. Harwood Academic Publishers, Chur, London, Paris, Utrecht, New York, 1984.

31. C.E. Kenig, Recent progress on boundary value problems on Lipschitz domains Pseudodifferential operators and Applications. Proc. Symp. Pure Math. 43(1985), 175-205.

32. M. Kohr, M. Lanza de Cristoforis, S.E. Mikhailov, W.L. Wendland, Integral potential method for transmission problem with Lipschitz interface in $\mathbb{R}^{3}$ for the Stokes and Darcy-Forchheimer-Brinkman PDE systems. Z. Angew. Math. Phys. 67:116 (2016), no. 5, 1-30, DOI: 10.1007/s00033-016-0696-1.

33. M. Kohr, M. Lanza de Cristoforis, W.L. Wendland, Nonlinear Neumann-transmission problems for Stokes and Brinkman equations on Euclidean Lipschitz domains, Potential Anal. 38 (2013), 1123-1171.

34. M. Kohr, M. Lanza de Cristoforis, W.L. Wendland, Nonlinear Darcy-Forchheimer-Brinkman system with linear Robin boundary conditions in Lipschitz domains. In: Complex Analysis and Potential Theory (T. Aliev Azeroglu, A. Golberg, S. Rogosin eds.), 111-124, Cambridge Scientific Publishers, 2014. ISBN 978-1-908106-40-7.

35. M. Kohr, M. Lanza de Cristoforis, W.L. Wendland, Boundary value problems of Robin type for the Brinkman and Darcy-ForchheimerBrinkman systems in Lipschitz domains. J. Math. Fluid Mech. 16 (2014), 595-630.

36. M. Kohr, M. Lanza de Cristoforis, W.L. Wendland, Poisson problems for semilinear Brinkman systems on Lipschitz domains in $\mathbb{R}^{3}$. Z. Angew. Math. Phys. 66 (2015), 833-864. 
37. M. Kohr, M. Lanza de Cristoforis, W.L. Wendland, On the Robin-transmission boundary value problems for the nonlinear DarcyForchheimer-Brinkman and Navier-Stokes systems. J. Math. Fluid Mech. 18 (2016), 293-329.

38. M. Kohr, D. Medková, W.L. Wendland, On the Oseen-Brinkman flow around an (m-1)-dimensional solid obstacle. Monatsh. Math. 183 (2017), 269-302.

39. M. Kohr, S.E. Mikhailov, W.L. Wendland, Transmission problems for the Navier-Stokes and Darcy-Forchheimer-Brinkman systems in Lipschitz domains on compact Riemannian manifolds. J. Math. Fluid Mech. 19 (2017), 203-238.

40. M. Kohr, I. Pop, Viscous Incompressible Flow for Low Reynolds Numbers. WIT Press, Southampton (UK), 2004.

41. L. Lanzani, L. Capogna, L.R.M. Brown, The mixed problem in $L^{p}$ for some two-dimentional Lipschitz domains. Math. Ann. 342 (2008), 91-124.

42. V. Maz'ya, J. Rossmann, Lp estimates of solutions to mixed boundary value problems for the Stokes system in polyhedral domains. Math. Nachr. 280 (2007), 751-793.

43. M. McCracken, The resolvent problem for the Stokes equations on half-space in $L_{p}^{*}$, SIAM J. Math. Anal. 12 (1981), $201-228$.

44. W. McLean, Strongly Elliptic Systems and Boundary Integral Equations. Cambridge University Press, Cambridge, UK, 2000.

45. D. Medková, Transmission problem for the Brinkman system, Complex Var. Elliptic Equ. 59 (2014), 1664-1678.

46. D. Medková, Bounded solutions of the Dirichlet problem for the Stokes resolvent system, Complex Var. Elliptic Equ. 61 (2016), 1689-1715.

47. D. Medková, $L^{q}$-solution of the Robin Problem for the Oseen System, Acta Appl. Math. 142 (2016), 61-79.

48. S. E. Mikhailov, Analysis of united boundary-domain integro-differential and integral equations for a mixed BVP with variable coefficient. Math. Methods Appl. Sci. 29 (2006) 715-739.

49. S.E. Mikhailov, Localized direct boundary-domain integro-differential formulations for scalar nonlinear boundary-value problems with variable coefficients, J. Eng. Math. 51 (2005), 283-302.

50. S.E. Mikhailov, Direct localized boundary-domain integro-differential formulations for physically nonlinear elasticity of inhomogeneous body, Engng. Anal. Bound. Elem. 29 (2005), 1008-1015.

51. S.E. Mikhailov, Traces, extensions and co-normal derivatives for elliptic systems on Lipschitz domains, J. Math. Anal. Appl. 378 (2011), 324-342.

52. S.E. Mikhailov, Solution regularity and co-normal derivatives for elliptic systems with non-smooth coefficients on Lipschitz domains, J. Math. Anal. Appl. 400 (2013), 48-67.

53. S.E. Mikhailov, Analysis of segregated boundary-domain integral equations for variable-coefficient Dirichlet and Neumann problems with general data. ArXiv:1509.03501, 2015, 1-32.

54. S.G. Mikhlin, Multidimensional Singular Integrals and Integral Equations. Pergamon, Oxford, 1965.

55. D. Mitrea, M. Mitrea, J. Pipher, Vector potential theory on nonsmooth domains in $\mathbb{R}^{3}$ and applications to electromagnetic scattering, J. Fourier Anal. Appl., 3 (1997), 131-192.

56. D. Mitrea, M. Mitrea, M. Taylor, Layer potentials, the Hodge Laplacian and global boundary problems in non-smooth Riemannian manifolds, Memoirs of the American Mathematical Society, 150, no. 713 (2001), vii+120.

57. I. Mitrea, M. Mitrea, The Poisson problem with mixed boundary conditions in Sobolev and Besov spaces in non-smooth domains, Trans. Amer. Math. Soc, 359 (2007), 4143-4182.

58. I. Mitrea, M. Mitrea, Multi-Layer Potentials and Boundary Problems for Higher-Order Elliptic Systems in Lipschitz Domains. Springer, Heidelberg, 2013.

59. I. Mitrea, M. Mitrea, M. Wright, Optimal estimates for the inhomogeneous problem for the bi-Laplacian in three-dimensional Lipschitz domains, J. Math. Sci. (New York). 172 (2011), 24-134.

60. M. Mitrea, S. Monniaux, M. Wright, The Stokes operator with Neumann boundary conditions in Lipschitz domains, J. Math. Sci. (New York). 176 (2011), 409-457.

61. M. Mitrea, M. Wright, Boundary value problems for the Stokes system in arbitrary Lipschitz domains, Astérisque. 344 (2012), viii+241.

62. M. Mitrea, M. Taylor, Navier-Stokes equations on Lipschitz domains in Riemannian manifolds, Math. Ann. 321 (2001), $955-987$.

63. M. Mitrea, M. Taylor, Potential theory on Lipschitz domains in Riemannian manifolds: Sobolev-Besov space results and the Poisson problem, J. Funct. Anal. 176 (2000), 1-79.

64. J. Nečas, Direct Methods in the Theory of Elliptic Equations. Springer, Berlin, Heidelberg, 2012. [English translation of Les Méthodes Directes en Théorie des Équations Elliptique. Masson, Paris, 1967.]

65. D. A. Nield, A. Bejan, Convection in Porous Media. Third Edition, Springer, New York, 2013.

66. T. Runst, W. Sickel, Sobolev spaces of fractional order, Nemytskij operators, and nonlinear partial differential equations. Walter de Gruyter, Berlin, 1996.

67. R. Russo, A. Tartaglione, On the Robin problem for Stokes and Navier-Stokes systems, Math. Models Methods Appl. Sci. 19 (2006), $701-716$.

68. R. Russo, A. Tartaglione, On the Navier problem for stationary Navier-Stokes equations, J. Diff. Equ. 251 (2011), $2387-2408$.

69. Z. Shen, Resolvent estimates in $L^{p}$ for the Stokes operator in Lipschitz domains, Arch. Rational Mech. Anal. 205 (2012), 395-424.

70. J.L. Taylor, K.A. Ott and R.M. Brown, The mixed problem in Lipschitz domains with general decompositions of the boundary. Trans. Amer. Math. Soc. 365 (2013), 2895-2930.

71. J. Toft, Continuity properties for modulation spaces, with applications to pseudo-differential calculus - I, J. Funct. Anal. 207 (2004), 399-429.

72. H. Triebel, Theory of Function Spaces. Birkhäuser, Basel, 1983.

73. H. Triebel, Function spaces in Lipschitz domains and on Lipschitz manifolds. Characteristic functions as pointwise multipliers, Rev. Mat. Complut. 15 (2002), 475-524.

74. W. Varnhorn, The Stokes Equations. Akademie Verlag, Berlin 1994.

75. G. Verchota, Layer potentials and regularity for the Dirichlet problem for Laplace's equation in Lipschitz domains, J. Funct. Anal. 59 (1984), 572-611. 